\title{
The Holomorphic Hörmander Functional Calculus
}

\author{
Dissertation \\ zur Erlangung des Doktorgrades \\ der Mathematisch-Naturwissenschaftlichen Fakultät \\ der Christian-Albrechts-Universität \\ zu Kiel
}

vorgelegt von

Florian Pannasch

Kiel,

August 2019 
Referent:

Korreferent:
Prof. Dr. Markus Haase Prof. Dr. Detlef Müller

Tag der mündlichen Prüfung: $\quad$ 14.10.2019

Zum Druck genehmigt:

14.10.2019

Dekan:
Prof. Dr. Frank Kempken 
Für meine Großväter Wilhelm Karl Fritz Lampe († 2007) und Karl Heinz Pannasch († 2016). 



\section{Abstract}

The topic of this thesis is functional calculus in connection with abstract multiplier theorems.

In 1960, Hörmander showed how the uniform boundedness of certain integral means of a function $m \in \mathrm{L}^{\infty}\left(\mathbb{R}^{d}\right)$ and its weak derivatives imply that $m$ yields a bounded $\mathrm{L}^{p}$-Fourier multiplier. Nowadays, this is known as the Hörmander multiplier theorem, sometimes Hörmander-Mikhlin multiplier theorem. A noteworthy detail is that a radial function $m(|\mathbf{x}|)$ satisfies Hörmander's condition if and only if $m\left(|\mathbf{x}|^{2}\right)$ does. Hence, Hörmander's theorem is also a result on the functional calculus of the negative Laplacian $-\Delta$.

Hörmander's result has inspired a lot of research, and authors have also proven similar results for other operators such as certain Schrödinger operators, Sublaplacians on Lie groups, and later certain differential operators on spaces of homogeneous type. For us, the work of Kriegler and Weis is of particular interest. Starting with the PhD thesis of Kriegler in 2009, they showed how abstract multiplier theorems can be proven in a more general context. Namely, considering a certain class of 0 -sectorial and 0-strip type operators on a general Banach space, one can construct an abstract Hörmander functional calculus based on the classical holomorphic calculus. Then, by using probalistic techniques from Banach space geometry involving so-called $R$-boundedness one can derive multiplier results in this generalized setting.

In 2001, García-Cuerva, Mauceri, Meda, Sjögren, and Torrea proved an abstract multiplier theorem for generators of symmetric contraction semigroups, where a bounded Hörmander calculus is inferred from growth conditions on the imaginary powers of the generator. As the considered operators need not be 0 -sectorial, this result is not covered by the methods of Kriegler and Weis. However, the result is based on Meda's earlier work, where he derived a bounded Hörmander if the given imaginary powers only grow polynomially fast. In this case, the operator is 0-sectorial, and Kriegler and Weis were able to recover the result while improving the order of the calculus.

In this thesis, we introduce a generalized class of Hörmander functions defined on strips and sectors. Based on this and the classical holomorphic calculus, we construct a holomorphic Hörmander calculus for a class of operators which may also have strip type or angle of sectoriality greater than zero. The main result is a generalization of the multiplier theorem of García-Cuerva et al. to Banach spaces of finite cotype and Banach spaces with Pisier's property $(\alpha)$, where we retain and even improve the order given by 
Kriegler and Weis for the 0-sectorial case. 


\section{Zusammenfassung}

Das Thema dieser Dissertation ist Funktionalkalkül in Verbindung mit abstrakten Multiplikatorsätzen.

In 1960 hat Hörmander gezeigt, wie die gleichmäßige Beschränktheit bestimmter Integralmittel einer Funktion $m \in \mathrm{L}^{\infty}\left(\mathbb{R}^{d}\right)$ und ihrer schwachen Ableitungen bereits liefert, dass $m$ ein beschränkter $\mathrm{L}^{p}$-Fourier-Multiplikator ist. Heutzutage ist dieser Satz bekannt als der Multiplikatorsatz von Hörmander, manchmal Multiplikatorsatz von HörmanderMikhlin. Ein besonderes Detail ist, dass eine radiale Funktion $m(|\mathbf{x}|)$ die gegebene Bedingung von Hörmander genau dann erfüllt, wenn es die Funktion $m\left(|\mathbf{x}|^{2}\right)$ tut. Damit ist der Satz von Hörmander auch ein Ergebnis über den Funktionalkalkül des negativen Laplaceoperators $-\Delta$.

Hörmanders Resultat hat viel Forschung inspiriert, und viele Autoren haben ähnliche Sätze auch für andere Operatoren beweisen können, wie zum Beispiel gewisse Schrödingeroperatoren, Sublaplaceoperatoren auf bestimmten Lie-Gruppen, und später gewisse Differentialoperatoren auf Räumen vom homogenen Typ. Von besonderem Interesse ist für uns die Arbeit von Kriegler und Weis. Beginnend mit Krieglers Dissertation in 2009, zeigten die beiden, wie abstrakte Multiplikatorsätze in einem allgemeineren Kontext bewiesen werden können. Nämlich kann man für eine bestimmte Klasse von 0sektoriellen und 0-Streifentyp Operatoren einen abstrakten Hörmanderkalkül basierend auf dem klassischen holomorphen Kalkül konstruieren. Multiplikatorsätze können dann mithilfe von probablistischen Mitteln abgeleitet werden. Hierbei spielt die sogenannte $R$-Beschränkheit eine wichtige Rolle.

In 2001 haben García-Cuerva, Mauceri, Meda, Sjögren und Torrea einen abstrakten Multiplikatorsatz für Erzeuger symmetrischer Kontraktionshalbgruppen bewiesen, der aus gewissen Wachstumsbedingungen an die imaginären Potenzen des Erzeugers einen beschränkten Hörmanderkalkül schließt. Da die auftretenden Operatoren nicht zwingend 0 -sektoriell sein müssen, wird dieses Ergebnis nicht von den Mitteln von Kriegler und Weis abgedeckt. Allerdings basiert der Satz auf einem früheren Resultat von Meda, in dem er einen beschränkten Hörmanderkalkül folgert, wenn die gegebenen imaginären Potenzen höchstens polynomiell wachsen. In diesem Fall ist der Operator 0-sektoriell, und Kriegler und Weis konnten Medas Ergebnis reproduzieren, wobei die Ordnung des Kalküls sogar verbessert wurde.

In dieser Arbeit führen wir eine verallgemeinerte Klasse von Hörmanderfunktioen auf Streifen und Sektoren ein. Basierend hierauf und auf den klassischen holomor- 
phen Funktionalkalkül wird einen abstrakter Hörmanderkalkül für eine gewisse Klasse von Streifentyp- und sektoriellen Operatoren konstruiert, deren Streifentyp oder Sektorialitätswinkel auch größer als null sein kann. Das Hauptresultat ist eine Verallgemeinerung des Multiplikatorsatzes von García-Cuerva et al. auf Banachräume mit endlichem Kotyp und Banachräume mit Pisiers Eigenschaft $(\alpha)$, wobei wir die verbesserte Ordnung von Kriegler und Weis aus dem 0-sektoriellen Fall erhalten und sogar verbessern. 


\section{Danksagungen}

Allen voran möchte ich gern meinem Betreuer Markus Haase danken. Markus, in den letzten Jahren bin ich nicht nur stark als Mathematiker gewachsen, sondern auch als Mensch. Und ich denke, du bist dafür zu einem großen Teil verantwortlich. Vielen Dank für eine wunderschöne Zeit in Kiel, die ich ohne dich nie gehabt hätte.

Ich danke Christoph Kriegler für seine anregenden Gespräche in Wuppertal zum Workshop des 21sten Internet Seminars 2018. Danke für deine Ermutigungen und dein reges Interesse an meinem Thema.

Ich danke all meinen Kollegen aus dem Kieler Mathematischen Seminar für ihre stetige Bereitschaft zur mathematischen Diskussion und Kaffeepause: Stefan Buschenhenke, Markus Nieß, Ljudevit Palle, Frederic Sommer, Sascha Trostorff und Mareike Wolff. Außerdem sei großer Dank an meine ehemaligen Kollegen Partrick Comdühr und Marcin Wnuk gerichtet. Die Arbeits- (und Freizeit)atmosphäre in Kiel ist eine ganz besondere. Und das ist euch zu verdanken.

Besonderer Dank gebürt meinen engsten Kollegen: Alexander Dobrick und Marco Peruzzetto. Alexander, du versprühst einen Forschungsdrang, der wirklich ansteckend ist. Das schätze ich sehr. Und Marco, du bist einer der besten Freunde und Bürokollegen, die man sich nur wünschen kann. Mit niemandem sonst rede ich so gern über die Mathematik und das Leben. Per me sei come un fratello.

Einen Extradank richte ich an Alexander, Ljudevit, Marco, Patrick und Stefan. Danke für das Korrekturlesen!

Danke an meine Familie. Mama! Papa! Guckt mal! Danke an meine Schwestern Anna und Martha und an meinen Bruder Mike. Auch wenn ich es nicht oft nach Neschwitz schaffe: Jedes mal wenn ich angekomme, fühl mich als wär ich erst gestern da gewesen. So eine warme Atmosphäre ist keine Selbstverständlichkeit. Danke, dass ihr immer für mich da seid.

Danke an meine zwei besten Freunde: Marcel Sporka und Ronny Wels. Danke für eure Unterstützung. In allen Lebenslagen.

$\mathrm{Zu}$ guter letzt danke ich noch meiner Freundin Jia Chen. Du bist der Grund, warum ich mich jeden Tag anstrenge, mein Bestes zu geben. Und du bist der Grund, warum ich auch durchhalten kann, wenn mich der Selbstzweifel plagt. Dafür danke ich dir von ganzem Herzen, Jia Jia. 


\section{Contents}

$\begin{array}{lll}1 & \text { Introduction } & 1\end{array}$

\begin{tabular}{|lll|}
\hline 2 & Preliminaries and Notation & $\mathbf{7}$
\end{tabular}

2.1 General Notation . . . . . . . . . . . . . . . . . . . . . . . . 8

2.2 The Abstract Functional Calculus . . . . . . . . . . . . . . . . . . . . 11

2.3 The Holomorphic Calculus for Strip Type Operators . . . . . . . . . . . . 13

2.4 The Holomorphic Calculus for Injective Sectorial Operators . . . . . . . . . 17

$2.5 \quad$ Groups of Operators and their Functional Calculus . . . . . . . . . . . . . . 20

2.6 Geometry of Banach Spaces . . . . . . . . . . . . . . . . . 24

$\begin{array}{lll}3 & \text { Generalized Hörmander and Sobolev Functions } & 29\end{array}$

3.1 Admissible Weights . . . . . . . . . . . . . . . . . . . . . . . . . 31

3.2 Generalized Sobolev Functions on a Strip . . . . . . . . . . . . . . . 35

3.3 Generalized Hörmander Functions on a Strip . . . . . . . . . . . . . . . . . . 44

3.4 Generalized Hörmander Functions on a Sector . . . . . . . . . . . . . . . . 56

\begin{tabular}{|lll}
4 & The Holomorphic Hörmander Functional Calculus & 59
\end{tabular}

4.1 The Extended Sobolev Functional Calculus . . . . . . . . . . . . . . . . 60

$4.2 \quad$ A More General Approach To Hörmander Functional Calculi . . . . . . . 69

4.3 Results on Bounded Hörmander Calculi . . . . . . . . . . . . . . . . . . 75

4.4 The Hörmander Calculus for Sectorial Operators . . . . . . . . . . . . . . 84

$\begin{array}{llr}5 & \text { Applications } & 89\end{array}$

$5.1 \quad$ Elliptic Partial Differential Operators with Constant Coefficients . . . . . 89

5.2 Generators of Symmetric Contraction Semigroups . . . . . . . . . . . . . 92

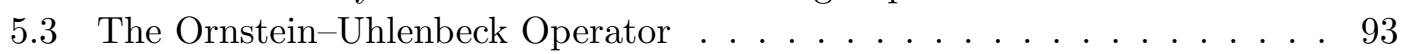

\begin{tabular}{ll}
\hline Bibliography & 95
\end{tabular}

\begin{tabular}{ll}
\hline Index & 99
\end{tabular} 


\section{Chapter 1}

\section{Introduction}

In 1960, Hörmander proved the following extension of Mikhlin's multiplier theorem (cf. [28, Theorem 2.5]).

Theorem 1.1.1 (Hörmander, 1960). Let $m \in \mathrm{L}^{\infty}\left(\mathbb{R}^{d}\right)$, let $N \in \mathbb{N}$ with $N>\frac{d}{2}$, and suppose that

$$
\left.\left.\sup _{R>0} \int_{\frac{R}{2}<|x|<2 R}|| x\right|^{|\alpha|} \cdot \mathrm{D}^{\alpha} m(x)\right|^{2} \frac{\mathrm{d} x}{|x|^{d}}<\infty
$$

for all $\alpha \in \mathbb{N}_{0}^{N}$ with $|\alpha| \leq N$. Then for every $p \in(1, \infty)$

$$
T_{m}: \mathcal{S}\left(\mathbb{R}^{d}\right) \rightarrow \mathrm{L}^{p}\left(\mathbb{R}^{d}\right), \quad T_{m} \varphi:=\mathcal{F}^{-1}(m \cdot \widehat{\varphi}),
$$

extends to a bounded operator on $\mathrm{L}^{p}\left(\mathbb{R}^{d}\right)$.

Here, $\mathcal{S}\left(\mathbb{R}^{d}\right)$ denotes the Schwartz space on $\mathbb{R}^{d}$. Note that Hörmander's condition (1.1.1) can be rewritten to

$$
\sup _{t>0}\|\eta(|\mathbf{x}|) \cdot m(t \mathbf{x})\|_{\mathrm{W}^{N, 2}\left(\mathbb{R}^{d}\right)}<\infty,
$$

where $\mathbf{x}$ denotes the mapping $x \mapsto x, \eta$ is a non-zero test function with support in $(0, \infty)$, and $\mathrm{W}^{N, 2}\left(\mathbb{R}^{d}\right)$ is the usual $\mathrm{L}^{2}$-Sobolev space on $\mathbb{R}^{d}$. One can show that 1.1 .2 does not depend on the particular choice of $\eta$. This reformulation has the advantage that it also makes sense for non-integer powers $N$ if one replaces the classical Sobolev space with a fractional Sobolev space. Moreover, Hörmander's proof in [28] actually yields that it is enough to require the function $m$ to satisfy $\left(1.1 .2\right.$ for some fractional power $N=\beta>\frac{d}{2}$.

When considering radial multipliers $m=f(|\mathbf{x}|)$, one can show that $m$ already satisfies 1.1.2 for $N=\beta>0$ if the function $f$ satisfies 1.1 .2 for the one-dimensional case. That is,

$$
\sup _{t>0}\|\eta \cdot f(t \mathbf{s})\|_{\mathrm{W}^{\beta, 2(\mathbb{R})}}<\infty
$$

where $\beta>0$, and $\eta$ is again a non-zero test function with support in $(0, \infty)$. Indeed, for $N \in \mathbb{N}$ this follows from a technical but straightforward argument involving Faà di 
Bruno's formula (see [9, (2.1)]) and the coarea formula for sperical coordinates (see [14, 3.2.13]). The case $N=\beta>0$ follows from an interpolation argument. Similarly, $f$ satisfies 1.1.3 if and only if $f\left(\mathbf{s}^{2}\right)$ does (see also the introduction of [7]). Hence, one arrives at the following corollary of Theorem 1.1.1.

Corollary 1.1.2. Let $f \in \mathrm{L}^{\infty}\left(\mathbb{R}_{+}\right)$, let $d \in \mathbb{N}$, let $\beta>\frac{d}{2}$, suppose that $f$ satisfies 1.1.3), and set $m:=f\left(|\mathbf{x}|^{2}\right)$. Then, for every $p \in(1, \infty)$,

$$
T_{m}: \mathcal{S}\left(\mathbb{R}^{d}\right) \rightarrow \mathrm{L}^{p}\left(\mathbb{R}^{d}\right), \quad T_{m} \varphi:=\mathcal{F}^{-1}(m \cdot \widehat{\varphi})=f(-\Delta) \varphi
$$

extends to a bounded operator on $\mathrm{L}^{p}\left(\mathbb{R}^{d}\right)$.

This result is also often referred to as the Hörmander multiplier theorem. In this form, Hörmander's theorem is a result on the functional calculus of the negative Laplacian $-\Delta$. Namely, if $\mathrm{Hör}^{\beta, 2}\left(\mathbb{R}_{+}\right)$denotes the space of all functions $f \in \mathrm{L}^{\infty}\left(\mathbb{R}_{+}\right)$satisfying 1.1.3), then Corollary 1.1 .2 states that $-\Delta$ has a bounded $\operatorname{Hör}^{\beta, 2}\left(\mathbb{R}_{+}\right)$-calculus for every $\beta>\frac{d}{2}$.

An interesting question is whether similar results can also be proven for other operators. That is, do other operators also admit a bounded $\mathrm{Hör}^{\beta, 2}\left(\mathbb{R}_{+}\right)$-calculus, and if yes, for which $\beta$ ? In the literature, one can find several results in this direction. Amongst others, there are multiplier theorems based on Hörmander's condition for special Schrödinger operators (see [25]), left-invariant sublaplacians on certain Lie groups (see e.g., [15, (6.25)], 77, [26], [44]), and specific differential operators on spaces of homogeneous type (see [54]).

One established approach to abstract multiplier theorems is to consider symmetric contraction semigroups (see [41], [11, [17, and [6] for example; see also [49] and [12]): Let $(\Omega, \mu)$ be a $\sigma$-finite measure space, let $\left(T_{t}\right)_{t \geq 0}$ be a symmetric contraction semigroup (see Definition 5.2.1), and let $-A$ denote the generator of $\left(T_{t}\right)_{t \geq 0}$. Write $\mathrm{L}^{p}:=\mathrm{L}^{p}(\Omega, \mu)$ for $p \in[1, \infty)$. On L $\mathrm{L}^{2}$, (the realization of) $A$ is a self-adjoint operator with spectrum in $\mathbb{R}_{+}$, so that for every $f \in \mathrm{L}^{\infty}\left(\mathbb{R}_{+}\right)$one can define the bounded operator $f(A)$ on $\mathrm{L}^{2}$ by means of the spectral theorem. Hence, for every $p \in[1, \infty)$, the operator $f(A)$ is also defined on the subspace $\mathrm{L}^{2} \cap \mathrm{L}^{p}$. As $\mathrm{L}^{2} \cap \mathrm{L}^{p}$ is dense in $\mathrm{L}^{p}$, the question whether $f(A)$ can be extended to a bounded operator on $\mathrm{L}^{p}$ reduces to the question whether there is a constant $c>0$ with

$$
\|f(A) x\| \leq c\|x\|_{p} \quad\left(x \in \mathrm{L}^{2} \cap \mathrm{L}^{p}\right) .
$$

At this point, usually more is known about the operator $A$ such as the explicit structure of $A$ or additional assumptions on operator families related to $A$. For example, Meda obtained the following result (cf. [41, Theorem 4]).

Theorem 1.1.3 (Meda, 1990). Let $-A$ be the injective generator of a symmetric contraction semigroup, let $p \in(1, \infty)$, and suppose there are constants $c, \alpha \geq 0$ with

$$
\left\|A^{-\mathrm{i} s} u\right\|_{p} \leq c(1+|s|)^{\alpha}\|u\|_{p} \quad\left(s \in \mathbb{R}, u \in \mathrm{L}^{p}\right) .
$$

Then $f(A)$ extends to a bounded operator on $\mathrm{L}^{p}$ for all $f \in \mathrm{Hör}^{\beta, 2}\left(\mathbb{R}_{+}\right)$with $\beta>\alpha+1$. 
The condition on $A$ to be injective is purely technical. Non-injective operators can be treated as well.

The big advantage of working with symmetric contraction semigroups is that a functional calculus is readily available for the operators $A$, as the spectral theorem provides a very natural way of defining operators $f(A)$ for all functions $f \in \mathrm{L}^{\infty}\left(\mathbb{R}_{+}\right)$. However, if one seeks to leave the $\mathrm{L}^{p}$-setting behind to consider operators on more general Banach spaces, the question for a suitable functional calculus becomes more difficult.

One possible way was outlined by Kriegler and Weis (see [32], [35], and [36]). They considered 0-sectorial and 0-strip type operators $A$ on a general Banach space $X$. For these operators a canonical calculus is given by the holomorphic functional calculus, and Kriegler and Weis showed how under certain conditions on $A$ an abstract Hörmander functional calculus can be constructed from the holomorphic calculus. In order to prove a multiplier theorem in this generalized setting, additional assumptions on the space $X$ and the operator $A$ are needed. Namely, $X$ needs to satisfy geometric conditions such as finite (Rademacher) cotype or Pisier's property $(\alpha)$ (see Section 2.6), and $A$ should have bounded $\mathrm{H}^{\infty}$-calculus. Then one can use probabilistic methods from Banach space geometry to infer a bounded Hörmander calculus for $A$. Here, the notion of so-called $R$-boundedness plays a key role. In this way, Kriegler and Weis were able to recover and even improve Meda's result (cf. [36, Theorem 6.1.(2)]).

Theorem 1.1.4 (Kriegler, Weis, 2018). Let $A$ be a 0-sectorial operator with bounded imaginary powers on a Banach space $X$. Let $X$ be of type $p \in[1,2]$ and of cotype $q \in[2, \infty)$ such that $\frac{1}{2}>\frac{1}{p}-\frac{1}{q}$. Further suppose that $A$ has a bounded $\mathrm{H}^{\infty}\left(\mathrm{S}_{\theta}\right)$-calculus for some angle $\theta \in(0, \pi)$, and that there are constants $c, \alpha \geq 0$ with

$$
\left\|A^{-\mathrm{i} s}\right\| \leq c(1+|s|)^{\alpha} \quad(s \in \mathbb{R}) .
$$

Then $A$ has a bounded $\mathrm{Hör}^{\beta, 2}\left(\mathbb{R}_{+}\right)$-calculus for all $\beta>\alpha+\frac{1}{2}$. If, in addition, $X$ has Pisier's property $(\alpha)$, then the calculus is $R$-bounded.

The formulation given in [36] is actually more general.

Here, we mean the Rademacher type and cotype of $X$. Moreover, $\mathrm{S}_{\theta}$ denotes the sector in the complex plane with opening angle $\theta$ and vertex at the origin, and $\mathrm{H}^{\infty}\left(\mathrm{S}_{\theta}\right)$ is the space of all bounded holomorphic functions on $\mathrm{S}_{\theta}$.

The hypothesis of Theorem 1.1.4 does indeed cover the setting in [41]: Let $A$ be the generator of a symmetric contraction semigroup. As a consequence of a celebrated result of Prüss and Sohr (see [23, Corollary 4.3.4]), if the imaginary powers of $A$ grow at most polynomially, then $A$ is 0 -sectorial. Moreover, by a result of Cowling (cf. [12, Theorem 2]), $A$ has a bounded $\mathrm{H}^{\infty}\left(\mathrm{S}_{\theta}\right)$-calculus for every angle $\theta \in\left(\frac{\pi}{2}, \pi\right)$. Finally, if $X=\mathrm{L}^{p}$ for $p \in(1, \infty)$, then $X$ satisfies the geometric assumptions of Theorem 1.1.4 (see Remark $2.6 .4 .4)$ ).

Kriegler and Weis improved Meda's result in two aspects. Namely, the order of the obtained calculus was improved by reducing the "gap" between $\beta$ and $\alpha$ from 1 to $\frac{1}{2}$, 
and the calculus was shown to be even $R$-bounded.

In 2001, García-Cuerva, Mauceri, Meda, Sjögren, and Torrea extended Meda's earlier result in a different way (cf. [17, Theorem 2.2]).

Theorem 1.1.5 (García-Cuerva et al., 2001). Let $-A$ be an injective generator of a symmetric contraction semigroup, let $p \in(1, \infty)$, and suppose there are constants $c, \alpha \geq$ 0 and an angle $\omega \in\left[0, \frac{\pi}{2}\right)$ with

$$
\left\|A^{-\mathrm{i} s}\right\| \leq c(1+|s|)^{\alpha} \mathrm{e}^{\omega|s|} \quad(s \in \mathbb{R}) .
$$

Then $A$ has a bounded $\mathrm{H}^{\infty}\left(\overline{\mathrm{S}}_{\omega}^{*} ; \beta\right)$-calculus for all $\beta>\alpha+1$.

Here, $\mathrm{H}^{\infty}\left(\overline{\mathrm{S}}_{\omega}^{*} ; \beta\right)$ is the notation from [17] for the space of all functions $f \in \mathrm{H}^{\infty}\left(\mathrm{S}_{\omega}\right)$ satisfying a holomorphic Hörmander condition of order $\beta$. Let us elaborate on that. Let $f \in \mathrm{H}^{\infty}\left(\mathrm{S}_{\omega}\right)$. By Fatou's theorem, $f$ admits a non-tangential limit almost everywhere on the boundary of $\mathrm{S}_{\omega}$, so that the functions $f\left(\mathrm{e}^{ \pm \mathrm{i} \omega} \mathbf{s}\right)$ are well-defined elements of $\mathrm{L}^{\infty}\left(\mathbb{R}_{+}\right)$. The function $f$ belongs to $\mathrm{H}^{\infty}\left(\mathrm{S}_{\omega} ; \beta\right)$ if both $f\left(\mathrm{e}^{ \pm \mathrm{i} \omega} \mathbf{s}\right)$ satisfy the classical Hörmander condition (1.1.3), that is,

$$
\sup _{t>0}\left\|\eta \cdot f\left(t \mathrm{e}^{ \pm \mathrm{i} \omega} \mathbf{s}\right)\right\|_{\mathrm{W}^{\beta, 2}(\mathbb{R})}<\infty
$$

where $\eta$ is again an arbitrary non-zero test function with support in $(0, \infty)$.

Theorem 1.1.5 was recently used by Carbonaro and Dragičević to prove that every generator of a symmetric contraction semigroup admits a bounded Hörmander calculus on a certain sector (cf. [6, Theorem 1]).

Remark 1.1.6. Although we exclusively investigate holomorphic Hörmander conditions in this thesis, it should be pointed out that one can also find holomorphic extensions of the classical Mikhlin condition (cf. [42, Theorem 2]) in the literature. The pioneer work for this was done by Clerc and Stein in [8]. See also [48], [3], [53], and the recent article [30]. Anker also considered more general holomorphic Mikhlin conditions, see [2, Theorem 20].

As the operators considered in Theorem 1.1.5 need not be 0 -sectorial anymore, this result does not fit into Kriegler and Weis's framework. Thus, a natural question is, whether the methods of Kriegler and Weis can be extended to operators with angle of sectoriality greater than zero, and if so, whether one can prove a similar result to Theorem 1.1 .4 for the non-zero case.

In this thesis, we give a positive answer to this. We construct a holomorphic Hörmander calculus that also admits operators of strip type or angle of sectoriality greater than zero. This construction is based on the classical holomorphic calculus and agrees with the calculus of Kriegler and Weis in the 0-strip type and 0-sectorial case. The main result of this thesis is the following theorem. 
Theorem 1.1.7. Let $X$ be a Banach space of type $p \in[1,2]$ and cotype $q \in[2, \infty)$, and let $A$ be a sectorial operator with bounded imaginary powers on $X$. Suppose that there is a measurable function $v_{\mathrm{BIP}}: \mathbb{R} \rightarrow(0, \infty)$ and a constant $\omega \in[0, \pi)$ such that

$$
\left\|A^{-\mathrm{i} s}\right\| \leq v_{\operatorname{BIP}}(s) \mathrm{e}^{\omega|s|}
$$

for all $s \in \mathbb{R}$. Further suppose there is $\theta \in(0, \pi)$ such that $A$ has a bounded $\mathrm{H}^{\infty}\left(\mathrm{S}_{\theta}\right)$ calculus. Let $r \in[1,2]$ with $\frac{1}{r}>\frac{1}{p}-\frac{1}{q}$, and let $v: \mathbb{R} \rightarrow[1, \infty)$ be an admissible weight with $\frac{v_{\mathrm{BIP}}}{v} \in \mathrm{L}^{r}(\mathbb{R})$. Then $A$ has a bounded $\operatorname{Hör}_{v}^{2}\left(\overline{\mathrm{S}}_{\omega}^{*}\right)$-calculus. If, in addition, $X$ has Pisier's property $(\alpha)$, the calculus is R-bounded.

Here, Hör ${ }_{v}^{2}\left(\overline{\mathrm{S}}_{\omega}^{*}\right)$ denotes the generalized Hörmander space on the sector

$$
\overline{\mathrm{S}}_{\omega}^{*}:=\overline{\mathrm{S}_{\omega}} \backslash\{0\}
$$

with respect to the admissible weight $v$ (see Section 3.4 , for the definition of an admissible weight, see Definition 3.1.1. For a polynomial weight $v=(1+|\mathbf{s}|)^{\beta}$ this space coincides with space $\mathrm{H}^{\infty}\left(\mathrm{S}_{\omega} ; \beta\right)$ considered in [17]. Theorem 1.1.7 is a generalization of [17, Theorem 2.2] which retains the improved order given by Kriegler and Weis for the 0 -sectorial case.

The biggest difficulty of proving Theorem 1.1.7 lies in the abstract background, or more precisely, in defining the holomorphic Hörmander calculus for operators with strip type or angle of sectoriality greater than zero. The reason for this is that the construction of Kriegler and Weis is not readily extendible to the non-zero case due to a heavy reliance on test functions. To better explain this, let us first review the construction given in [35]. Let $A$ be a 0 -sectorial operator with dense domain and range on a Banach space $X$, and suppose that $A$ satisfies the condition

$$
\int_{\mathbb{R}}\left|\frac{1}{(1+|s|)^{\beta}}\left\langle A^{-\mathrm{i} s} \rho_{\theta}(A) x, x^{\prime}\right\rangle\right|^{2} \mathrm{~d} s \lesssim\|x\|^{2}\left\|x^{\prime}\right\|^{2}
$$

for all $x \in X$ and $x^{\prime} \in X^{\prime}$, where $\beta>\frac{1}{2}, \theta>0$, and $\rho_{\theta}(A):=A^{\theta}(\mathrm{I}+A)^{-2 \theta}$ (cf. [35, $(3.9)])$. From (1.1.5) one can construct a (possibly unbounded) Sobolev calculus based on the holomorphic calculus of $A$ (we omit the details at this point). In particular, one may "insert" $A$ into test functions on $(0, \infty)$, that is, the operators

$$
\eta(A) \quad\left(\eta \in \mathrm{C}_{\mathrm{c}}^{\infty}\left(\mathbb{R}_{+}\right)\right)
$$

are well-defined (on the subspace $\operatorname{ran} \rho_{\theta}(A)$ ). Now, fix a dyadic partition of unity $\left(\varphi_{n}\right)_{n \in \mathbb{Z}}$ (see [35, Section 2.2]), choose $\theta>0$ as in (1.1.5), and consider the set

$$
D_{A}:=\left\{\sum_{k=-n}^{n} \varphi_{k}(A) x \mid n \in \mathbb{N}, x \in \operatorname{ran} \rho_{\theta}(A)\right\}
$$


(cf. [35, (3.13)]). This turns out to be a dense subspace of $X$. Now, for a function $f \in \operatorname{Hör}^{\beta, 2}\left(\mathbb{R}_{+}\right)$, the operator $f(A)$ is initially defined on $D_{A}$ by setting

$$
f(A)\left(\sum_{k=-n}^{n} \varphi_{k}(A) x\right):=\sum_{k=-n}^{n}\left(f \varphi_{k}\right)(A) x
$$

for $n \in \mathbb{N}$ and $x \in \operatorname{ran} \rho_{\theta}(A)$. Note that the expressions $\left(f \varphi_{k}\right)(A) x$ are well-defined, since $f \varphi_{k} \in \mathrm{W}^{\beta, 2}(\mathbb{R})$ for every $k \in \mathbb{Z}$ by condition 1.1.3. The operator in 1.1.7 is closable in $X$ (see [35, Lemma 3.11]), and $f(A)$ is defined as the closure of this operator in $X$. In this construction, test functions play a central role as the universal regularizers of Hörmander functions. However, if $A$ is sectorial with spectrum in a larger sector, then there is no way to define the operators $\eta(A)$ for $\eta \in \mathrm{C}_{\mathrm{c}}^{\infty}\left(\mathbb{R}_{+}\right)$in a reasonable way. Thus, a different approach is needed.

We show that instead of test functions one can also build the Hörmander calculus around a suitable class of holomorphic functions, namely $\mathrm{H}_{0}^{\infty}\left[\mathrm{S}_{\omega}\right]$ for sectorial operators and $\mathrm{H}_{0}^{\infty}\left[\mathrm{St}_{\omega}\right]$ for strip type operators (see Definitions 2.4.2 and 2.3.2). These function spaces have the advantage, that they are accessible with the holomorphic calculus of every sectorial, resp. strip type operator. And it is this shift from test functions to holomorphic functions which allows one to pass from the 0-sectorial and 0-strip type case to the non-zero case.

Similarly to Kriegler and Weis, we still need the additional requirement that an operator $A$ admits a (holomorphic) Sobolev calculus. For the most part, we restrict ourselves to the case where $A$ admits a bounded Sobolev calculus. Then, using a general principle from functional calculus theory, the so-called algebraic extension procedure, the Sobolev calculus is extended to a Hörmander calculus. This approach differs from the one in [35], as the Hörmander calculus is obtained by algebraic means rather than topological means. Although a topological extension argument is still needed, it is confined to the Sobolev calculus itself.

This approach to the Hörmander calculus requires a rigorous theory of holomorphic Hörmander and Sobolev functions on strips and sectors, which lies at the heart of this thesis (see Chapter 3).

At this point, we give an outline of the thesis. This is kept brief however, as there is a short overview of contents at the beginning of each chapter.

In Chapter 2, we fix the notation used throughout the rest of the text. Here, Sections 2.2 - 2.6 mostly prepare Chapters 4 and 5 , not so much Chapter 3.

In Chapter 3, we extensively discuss the scalar theory on which the holomorphic Hörmander functional calculus is based.

The actual operator theory and construction of the Hörmander calculus is given in Chapter 4. Here we also prove the main result.

In Chapter 5, we give applications of the main result to three different cases: elliptic partial differential operators with constant coefficients, generators of symmetric contraction semigroups, and the Ornstein-Uhlenbeck operator. 


\section{Chapter 2}

\section{Preliminaries and Notation}

In this chapter, we prepare the (possibly non-standard) notation used throughout the thesis and also give the necessary background for Chapter 4 and, to some extent, Chapter 5 .

To give a short overview, Section 2.1 introduces notation for

- sets of special interest (strips, sectors, positive real numbers),

- basic operator theory and Banach spaces,

- general estimates (for when we do not wish to keep track of certain constants),

- classical function spaces, norms, and operators (e.g. the Fourier transform),

- the manipulation of functions (e.g. restrictions to certain lines), and

- a selection of special functions.

The contents of this section mostly prepare Chapter 3 .

In Section 2.2, we fix the abstract framework for the functional calculus theory. Sections 2.3 and 2.4 give a short introduction to the classical holomorphic calculus of strip type and sectorial operators, and Section 2.5 briefly discusses the functional calculus of $C_{0}$-groups. Our main reference for these sections are [23] and [24]. Sections 2.2, 2.3 and 2.5 prepare Sections 4.1, 4.2 and partially 4.3 , while Section 2.4 is needed for Section 4.4 .

In Section 2.6 we recall four important notions from Banach space geometry: (Rademacher) type, (Rademacher) cotype, Pisier's property $(\alpha)$, and $\gamma$-boundedness. We also state results on how to obtain $\gamma$-bounded sets or $\gamma$-bounded homomorphisms. The main reference is [31]. Section 2.6 prepares the contents of Sections 4.3, 4.4, and Chapter 5.

Sections 2.2 - 2.6 are not needed for Chapter 3, with the exception of Definitions 2.3 .2 and 2.4.2. 


\subsection{General Notation}

Below we give an overview of the general notation used throughout the text.

\section{Notation for Special Sets}

By $\mathbb{N}$ we denote the set of all positive integers, i.e., $\mathbb{N}=\{1,2,3, \ldots\}$, while $\mathbb{N}_{0}=\mathbb{N} \cup\{0\}$ is the set of all nonnegative integers. We write $\mathbb{R}_{+}:=(0, \infty)$ for the open positive real line. Let $\omega>0$. Then

$$
\operatorname{St}_{\omega}:=\{z \in \mathbb{C}|| \operatorname{Im} z \mid<\omega\}
$$

denotes the strip of width $2 \omega$ with symmetry axis $\mathbb{R}$. For $\omega \in(0, \pi)$ we write

$$
\mathrm{S}_{\omega}:=\{z \in \mathbb{C} \backslash\{0\}|| \arg z \mid<\omega\}
$$

for the sector with symmetry axis $\mathbb{R}_{+}$and vertex at the origin. For convenience, we also set $\mathrm{St}_{0}:=\mathbb{R}$ and $\mathrm{S}_{0}:=\mathbb{R}_{+}$.

\section{General Notation for Banach Spaces}

For a Banach space $X$ the dual space is denoted by $X^{\prime}$, the norm on $X$ is denoted by $\|\cdot\|_{X}$, and for the dual pairing we write $\langle\cdot, \cdot \cdot\rangle$ (without reference to $X$, since the considered Banach space will always be clear from context). For a Hilbert space $H$ the notation $(\cdot \mid \cdot)_{H}$ is used for the inner product on $H$.

Let $X, Y$ be Banach spaces. Then $X \oplus Y$ denotes the Cartesian product of $X$ and $Y$ equipped with the norm

$$
\|(x, y)\|_{X \oplus Y}:=\|x\|_{X}+\|y\|_{Y} \quad(x \in X, y \in Y) .
$$

Let $A$ be a possibly unbounded linear operator between two Banach spaces $X$ and $Y$. We identify $A$ with its graph in $X \oplus Y$. By $\operatorname{dom} A, \operatorname{ran} A, \operatorname{ker} A, \sigma(A)$ and $\rho(A)$ we denote the domain, the range, the null space or kernel, the spectrum and the resolvent set of $A$, respectively. Furthermore, the notation " $A x=y$ " is used as a short hand for $(x, y) \in A$, i.e., the notation tacitly includes that $x \in \operatorname{dom} A$. Whenever $\lambda \in \rho(A)$, we write $R(\lambda, A):=(\lambda-A)^{-1}$ for the resolvent operator of $A$ in $\lambda$.

Let $X, Y, Z$ be Banach spaces, let $A, B$ be linear operators between $X$ and $Y$, and let $C$ be a linear operator between $Y$ and $Z$. The sum of $A$ and $B$ is defined as

$$
A+B:=\{(x, y) \in X \oplus Y \mid x \in \operatorname{dom} A \cap \operatorname{dom} B, y=A x+B x\},
$$

and the composition $C A$ is defined as

$$
C A:=\{(x, z) \in X \oplus Z \mid x \in \operatorname{dom} A, A x \in \operatorname{dom} C, z=C A x\} .
$$

For a Banach space $X$, we write $\mathcal{C}(X)$ for the set of all closed linear operators on $X$. The space $\mathcal{L}(X)$ denotes the algebra of all bounded operators on $X$, and $\mathrm{I}=\mathrm{I}_{X}$ is the identity operator on $X$. 


\section{General Notation for Estimates}

For real numbers $a, b \in \mathbb{R}$ and objects $F_{1}, \ldots, F_{n}$ we write

$$
a \lesssim F_{1}, \ldots, F_{n} b
$$

if there is a constant $c \geq 0$ only depending on $F_{1}, \ldots, F_{n}$ with $a \leq c b$. If both $a \lesssim_{F_{1}, \ldots, F_{n}} b$ and $b \lesssim_{F_{1}, \ldots, F_{n}} a$ we write $a \bar{\sim}_{F_{1}, \ldots, F_{n}} b$.

\section{Classical Function Spaces}

For a measure space $(\Omega, \mu)$, a Banach space $X$, and $p \in[1, \infty]$, the notations $\mathrm{L}^{p}(\Omega, \mu)$ and $\mathrm{L}^{p}(\Omega, \mu ; X)$ are used for the classical Lebesgue and Bochner spaces. On both spaces, the norm is denoted by $\|\cdot\|_{p}$. In the case that $(\Omega, \mu)=(\mathbb{R}, \mathrm{d} x)$, we also write $\mathrm{L}^{p}(\mathbb{R})$ and $\mathrm{L}^{p}(\mathbb{R} ; X)$ instead of $\mathrm{L}^{p}(\mathbb{R}, \mathrm{d} x)$ and $\mathrm{L}^{p}(\mathbb{R}, \mathrm{d} x ; X)$.

For a function $f: O \rightarrow \mathbb{C}$, where $O$ is some non-empty set, we write

$$
\|f\|_{\infty, O}:=\sup _{s \in O}|f(s)|
$$

for the supremum norm of $f$ on $O$. For $O \subseteq \mathbb{C}$ the set $\operatorname{supp}(f)$

$$
\operatorname{supp}(f):=\overline{\{z \in O \mid f(z) \neq 0\}}
$$

denotes the support of $f$.

Now fix a non-empty set $O \subseteq \mathbb{C}$. The following spaces of continuous functions are of interest for us:

$$
\begin{aligned}
\mathrm{C}_{\mathrm{b}}(O) & :=\{f: O \rightarrow \mathbb{C} \mid f \text { is continuous and bounded }\}, \\
\mathrm{C}_{\mathrm{c}}(O) & :=\left\{f \in \mathrm{C}_{\mathrm{b}}(O) \mid \operatorname{supp}(f) \text { is compact }\right\}, \\
\mathrm{C}_{0}(O) & :=\overline{\mathrm{C}_{\mathrm{c}}(O)}\|\cdot\|_{\infty, O}, \\
\mathrm{UC}_{\mathrm{b}}(O) & :=\left\{f \in \mathrm{C}_{\mathrm{b}}(O) \mid f \text { is uniformly continuous }\right\},
\end{aligned}
$$

where each space is equipped with the supremum norm. If $O \subseteq \mathbb{R}$ is open in $\mathbb{R}$ and $k \in \mathbb{N}$, then $\mathrm{C}^{k}(O)$ denotes the space of all $k$-times continuously differentiable functions $f: O \rightarrow \mathbb{C}$ and $\mathrm{C}_{\mathrm{c}}^{k}(O):=\mathrm{C}^{k}(O) \cap \mathrm{C}_{\mathrm{c}}(O)$.

Now suppose that $O \subseteq \mathbb{C}$ is non-empty and open in $\mathbb{C}$. Then we consider

$$
\begin{aligned}
& \operatorname{Hol}(O):=\{f: O \rightarrow \mathbb{C} \mid f \text { is holomorphic }\}, \\
& \mathrm{H}^{\infty}(O):=\{f \in \operatorname{Hol}(O) \mid f \text { is bounded }\}=\operatorname{Hol}(O) \cap \mathrm{C}_{\mathrm{b}}(O), \\
& \mathrm{H}^{\infty}(\bar{O}):=\operatorname{Hol}(O) \cap \mathrm{C}_{\mathrm{b}}(\bar{O}),
\end{aligned}
$$

and equip both spaces $\mathrm{H}^{\infty}(O)$ and $\mathrm{H}^{\infty}(\bar{O})$ with the supremum norm. 


\section{Notation for Special Functions}

For subsets $D \subseteq \mathbb{R}, O \subseteq \mathbb{C}$, and $U \subseteq \mathbb{R}^{d}$ we write

$$
\mathbf{s}: D \rightarrow \mathbb{R}, \quad s \mapsto s, \quad \mathbf{x}: U \rightarrow \mathbb{R}^{d}, \quad x \mapsto x, \quad \text { and } \quad \mathbf{z}: O \rightarrow \mathbb{C}, \quad z \mapsto z .
$$

The sets $D, O$, and $U$ will always be clear in the given context and thus not further referred to.

For a subset $D \subseteq \mathbb{R}$ the corresponding indicator function is denoted by $\mathbb{1}_{D}: \mathbb{R} \rightarrow \mathbb{R}$.

Moreover, the notation $\psi$ is exclusively used for the function

$$
\psi: \mathbb{C} \rightarrow \mathbb{C}, \quad \psi(z):=\mathrm{e}^{-z^{2}} .
$$

\section{Restrictions to Lines and Holomorphic Conjugates}

Let $O \subseteq \mathbb{C}$ and let $f: O \rightarrow \mathbb{C}$ be a function. If there is $r \in \mathbb{R}$ with ir $+\mathbb{R} \subseteq O$, then we use the notation

$$
f_{r}:=f(\mathbf{s}+\mathrm{i} r)
$$

for the restriction of $f$ on the line ir $+\mathbb{R}$. If $\bar{z} \in O$ for every $z \in O$, we write

$$
f^{*}:=\overline{f(\overline{\mathbf{z}})} \text {. }
$$

Note that $f$ is holomorphic if and only if $f^{*}$ is holomorphic.

\section{Translation Operators}

Let $X$ be a space of functions $f: O \rightarrow \mathbb{C}$ where $O \subseteq \mathbb{C}$. Suppose there is $w \in \mathbb{C}$ with $w+O \subseteq O$. Then,

$$
\tau_{w}: X \rightarrow X, \quad \tau_{w} f:=f(\mathbf{z}-w)
$$

denotes the complex shift by $w$ on $X$. In particular, if $O=\mathbb{R}$ and $\omega=t \in \mathbb{R}$, then $\tau_{t}$ is the usual right shift operator.

\section{Convolution and the Fourier Transform}

For functions $f, g: \mathbb{R} \rightarrow \mathbb{C}$,

$$
(f * g)(t):=\int_{\mathbb{R}} f(t-s) g(s) \mathrm{d} s \quad(t \in \mathbb{R})
$$

denotes the convolution of $f$ and $g$, whenever the integral above is well defined. For a function $f \in \mathrm{L}^{1}(\mathbb{R})$, we write

$$
(\mathcal{F} f)(t):=\widehat{f}(t):=\int_{\mathbb{R}} f(s) \mathrm{e}^{-\mathrm{i} s t} \mathrm{~d} s \quad(t \in \mathbb{R})
$$

for the Fourier transform of $f$, and

$$
\left(\mathcal{F}^{-1} f\right)(t):=f^{\vee}(t):=\frac{1}{2 \pi} \int_{\mathbb{R}} f(s) \mathrm{e}^{\mathrm{i} s t} \mathrm{~d} s \quad(t \in \mathbb{R})
$$

for the inverse Fourier transform of $f$. Whenever $f$ is defined on a set $O \subseteq \mathbb{C}$ with $\mathbb{R} \subseteq O$, then $\mathcal{F} f:=\widehat{f}:=\mathcal{F}\left(\left.f\right|_{\mathbb{R}}\right)$ and $\mathcal{F}^{-1} f:=f^{\vee}:=\mathcal{F}^{-1}\left(\left.f\right|_{\mathbb{R}}\right)$. 


\subsection{The Abstract Functional Calculus}

In this section, we formulate the abstract framework for the functional calculus theory. For more details see [24, Chapter 2 and Chapter 7].

In the following, let $\mathcal{F}$ be a unital commutative algebra with unit 1 , let $\mathcal{E} \subseteq \mathcal{F}$ be a subalgebra, and let $\Phi: \mathcal{E} \rightarrow \mathcal{L}(X)$ be an algebra homomorphism for some complex Banach space $X$. We do not require that $1 \in \mathcal{E}$.

Definition 2.2.1. A subset $\mathcal{E}^{\prime} \subseteq \mathcal{E}$ is called an anchor set (with respect to $\Phi$ ) if

$$
\bigcap_{e^{\prime} \in \mathcal{E}^{\prime}} \operatorname{ker} \Phi\left(e^{\prime}\right)=\{0\} \text {. }
$$

If $e \in \mathcal{E}$ is such that $\Phi(e)$ is injective, then $e$ is called an anchor element (with respect to $\Phi)$. The algebra homomorphism $\Phi$ is said to be non-degenerate if $\mathcal{E}$ itself is an anchor set with respect to $\Phi$.

Definition 2.2.2. Suppose that $\Phi$ is non-degenerate. An element $f \in \mathcal{F}$ is said to be anchored in $\mathcal{E}$ (with respect to $\Phi$ ) if the set

$$
[f]_{\mathcal{E}}:=\{e \in \mathcal{E} \mid e f \in \mathcal{E}\}
$$

is an anchor set. The space of all anchored elements is denoted by $[\mathcal{F}]_{\mathcal{E}}=[\mathcal{F}]_{\mathcal{E}, \Phi}$.

Note that for each $e^{\prime} \in \mathcal{E}$ one has $\left[e^{\prime}\right]_{\mathcal{E}}=\mathcal{E}$, and

$$
\Phi\left(e^{\prime}\right) x=y \quad \Longleftrightarrow \quad \forall e \in \mathcal{E}: \quad \Phi(e) \Phi\left(e^{\prime}\right) x=\Phi\left(e e^{\prime}\right) x=\Phi(e) y \quad(x, y \in X),
$$

since $\mathcal{E}$ is an anchor set. Consequently, the next definition does not cause any ambiguity.

Definition 2.2.3. For $f \in[\mathcal{F}]_{\mathcal{E}}$ one defines the (possibly unbounded) linear operator $\Phi(f)$ via setting

$$
\Phi(f) x=y \quad \stackrel{\mathrm{def}}{\Longleftrightarrow} \forall e \in[f]_{\mathcal{E}}: \quad \Phi(e f) x=\Phi(e) y .
$$

This procedure of extending the homomorphism $\Phi: \mathcal{E} \rightarrow \mathcal{L}(X)$ to the mapping

$$
\Phi:[\mathcal{F}]_{\mathcal{E}} \rightarrow\{A \subseteq X \oplus X \mid A \text { linear operator }\}
$$

is called the algebraic extension procedure, and the extension is called the algebraic extension of $\Phi$ (with respect to $\mathcal{F}$ ). In this context, $\Phi: \mathcal{E} \rightarrow \mathcal{L}(X)$ is called the elementary calculus, sometimes primary calculus.

\section{Remarks 2.2.4.}

1) Every functional calculus in this thesis fits into Definition 2.2.3. That is, the main ingredient to any calculus is the elementary calculus. The larger calculus is then obtained by means of the algebraic extension (see Definitions 2.3.7, 4.1.7, and 4.2.5 for example). 
2) One does not have $\mathcal{F}=[\mathcal{F}]_{\mathcal{E}}$ in general.

3) Let $f \in[\mathcal{F}]_{\mathcal{E}}$, and let $\mathcal{M} \subseteq[f]_{\mathcal{E}}$ be an anchor set. A straightforward argument yields that

$$
\Phi(f) x=y \quad \Longleftrightarrow \quad \forall e \in \mathcal{M}: \quad \Phi(e f) x=\Phi(e) y .
$$

In particular, if there exists an anchor element $e \in[f]_{\mathcal{E}}$, then

$$
\Phi(f)=\Phi(e)^{-1} \Phi(e f) .
$$

It follows that our framework recovers the classical holomorphic calculus for unbounded operators introduced by McIntosh in [40] and extensively discussed by Haase in [23]. (See Example 2.3.8.2).)

Lemma 2.2.5. Suppose that $\Phi$ is non-degenerate. The space $[\mathcal{F}]_{\mathcal{E}}$ is a subalgebra of $\mathcal{F}$ with $1 \in[\mathcal{F}]_{\mathcal{E}}$.

Proof. It is clear that both $0,1 \in[\mathcal{F}]_{\mathcal{E}}$. Let $f, g \in \mathcal{F}$, and note that on one hand $[f]_{\mathcal{E}} \cdot[g]_{\mathcal{E}} \subseteq[f g]_{\mathcal{E}}$. On the other hand, whenever $\mathcal{E}^{\prime} \subseteq \mathcal{E}$ and $\mathcal{E}^{\prime \prime} \subseteq \mathcal{E}$ are anchor sets, then $\mathcal{E}^{\prime} \cdot \mathcal{E}^{\prime \prime}$ is an anchor set as well. Hence, $[f g]_{\mathcal{E}}$ is an anchor set whenever both $f$ and $g$ are anchored in $\mathcal{E}$.

The next proposition collects well-known properties of the extended calculus. For proofs and a detailed discussion see [24, Section 2.4 and Section 7.1].

Proposition 2.2.6. Let $\mathcal{E}, \mathcal{F}, X$ and $\Phi: \mathcal{E} \rightarrow \mathcal{L}(X)$ be as above, and suppose that $\Phi$ is non-degenerate. Also let $f, g \in[\mathcal{F}]_{\mathcal{E}}$, and $\lambda \in \mathbb{C}$. Then the following statements hold:

a) $\Phi(f)$ is a closed operator;

b) $\lambda \Phi(f) \subseteq \Phi(\lambda f)$;

c) $\Phi(f)+\Phi(g) \subseteq \Phi(f+g)$;

d) $\Phi(f) \Phi(g) \subseteq \Phi(f g)$ with $\operatorname{dom} \Phi(f) \Phi(g)=\operatorname{dom} \Phi(g) \cap \operatorname{dom} \Phi(f g)$;

e) $\operatorname{ran} \Phi(e) \subseteq \operatorname{dom} \Phi(f)$ for every $e \in[f]_{\mathcal{E}}$;

f) $\Phi(0)=0$ and $\Phi(1)=\mathrm{I}$.

The next definition introduces terminology used in our convergence lemma for the Hörmander functional calculus (Theorem 4.1.14).

Definition 2.2.7. A subset $\mathcal{E}^{\prime} \subseteq \mathcal{E}$ is said to have total range with respect to $\Phi$ if the set

$$
Y_{\mathcal{E}}:=\left\{\Phi\left(e^{\prime}\right) x \mid e^{\prime} \in \mathcal{E}^{\prime}, x \in X\right\}=\bigcup_{e^{\prime} \in \mathcal{E}^{\prime}} \operatorname{ran} \Phi\left(e^{\prime}\right)
$$

is total in $X$, that is, the linear span of $Y_{\mathcal{E}}$ is dense in $X$. 


\subsection{The Holomorphic Calculus for Strip Type Operators}

Now we give a brief overview of all relevant definitions and results regarding the classical holomorphic calculus for strip type operators, and we discuss how this calculus fits into the framework of Section 2.2 .

Throughout, let $X$ denote a complex Banach space.

Definition 2.3.1. A possibly unbounded operator $A \subseteq X \oplus X$ is called a strip type operator if there is $\omega \geq 0$ with

$$
\sigma(A) \subseteq \overline{\mathrm{St}}_{\omega} \quad \text { and } \sup _{\lambda \in \mathbb{C} \backslash \mathrm{St}_{\theta}}\|R(\lambda, A)\|<\infty \quad \text { for all } \quad \theta>\omega .
$$

For a strip type operator $A$, the number

$$
\omega_{\mathrm{st}}(A):=\inf \{\omega \geq 0 \mid 2.3 .1 \text { holds }\}
$$

is called the strip type of $A$. If $\omega_{\mathrm{st}}(A)=0$, then $A$ is also said to be of 0 -strip type.

In view of Section 2.2, every functional calculus has three ingredients:

- a unital function algebra $\mathcal{F}$ which contains all functions of interest (e.g., all holomorphic functions on a certain set $O \subseteq \mathbb{C}$ );

- an "elementary" algebra $\mathcal{E} \subseteq \mathcal{F}$; and

- a non-degenerate algebra homomorphism $\Phi: \mathcal{E} \rightarrow \mathcal{L}(X)$.

We determine $\mathcal{F}, \mathcal{E}$, and $\Phi$ for the holomorphic calculus of a strip type operator. To this end, consider the following function spaces.

Definition 2.3.2. Let $\omega>0$. The space

$$
\mathcal{E}\left(\mathrm{St}_{\omega}\right):=\left\{\psi \in \mathrm{H}^{\infty}\left(\mathrm{St}_{\omega}\right) \mid \forall r \in(-\omega, \omega): \quad \psi_{r}=\psi(\mathbf{s}+\mathrm{i} r) \in \mathrm{L}^{1}(\mathbb{R})\right\}
$$

is called the algebra of elementary functions on $\mathrm{St}_{\omega}$. We also define

$$
\mathrm{H}_{0}^{\infty}\left(\mathrm{St}_{\omega}\right):=\left\{\psi \in \mathrm{H}^{\infty}\left(\mathrm{St}_{\omega}\right)\left|\exists c_{0}, c_{1}>0: \quad\right| \psi \mid \leq c_{0} \mathrm{e}^{-c_{1}|\operatorname{Re} \mathbf{z}|}\right\} .
$$

For $\mathrm{F} \in\left\{\mathcal{E}, \mathrm{H}_{0}^{\infty}, \mathrm{H}^{\infty}, \mathrm{Hol}\right\}$ and $\omega^{\prime} \geq 0$ we write

$$
\mathrm{F}\left[\mathrm{St}_{\omega^{\prime}}\right]:=\bigcup_{\omega>\omega^{\prime}} \mathrm{F}\left(\mathrm{St}_{\omega}\right)
$$

Remark 2.3.3. Note that $\mathrm{H}_{0}^{\infty}\left(\mathrm{St}_{\omega}\right) \subseteq \mathcal{E}\left(\mathrm{St}_{\omega}\right) \subseteq \mathrm{H}^{\infty}\left(\mathrm{St}_{\omega}\right)$. Moreover, both $\mathrm{H}_{0}^{\infty}\left(\mathrm{St}_{\omega}\right)$ and $\mathcal{E}\left(\mathrm{St}_{\omega}\right)$ are algebraic ideals in $\mathrm{H}^{\infty}\left(\mathrm{St}_{\omega}\right)$ with respect to pointwise multiplication. In particular, both $\mathrm{H}_{0}^{\infty}\left(\mathrm{St}_{\omega}\right)$ and $\mathcal{E}\left(\mathrm{St}_{\omega}\right)$ are subalgebras of $\mathrm{H}^{\infty}\left(\mathrm{St}_{\omega}\right)$.

For the holomorphic calculus of an operator $A$ of strip type $\omega$, we choose $\mathcal{F}=\operatorname{Hol}\left[\mathrm{St}_{\omega}\right]$ and $\mathcal{E}=\mathcal{E}\left[\mathrm{St}_{\omega}\right]$. The homomorphism $\Phi$ is given in the next definition. 
Definition 2.3.4. Let $A$ be a strip type operator on $X$, and set $\omega:=\omega_{\text {st }}(A)$. For $\theta>\delta>\omega$ and $\psi \in \mathcal{E}\left(\mathrm{St}_{\theta}\right)$ define

$$
\begin{aligned}
\psi(A) & :=\frac{1}{2 \pi \mathrm{i}} \int_{\partial \mathrm{St}_{\delta}} \psi(z) R(z, A) \mathrm{d} z \\
& =\frac{1}{2 \pi \mathrm{i}}\left(\int_{\mathbb{R}} \psi(s-\mathrm{i} \delta) R(s-\mathrm{i} \delta, A) \mathrm{d} s-\int_{\mathbb{R}} \psi(s+\mathrm{i} \delta) R(s+\mathrm{i} \delta, A) \mathrm{d} s\right) .
\end{aligned}
$$

The mapping

$$
\Phi_{\mathrm{st}}:=\Phi_{\mathrm{st}, A}: \mathcal{E}\left[\mathrm{St}_{\omega}\right] \rightarrow \mathcal{L}(X), \quad \Phi_{\mathrm{st}}(\psi):=\psi(A)
$$

is called the elementary (holomorphic) calculus of $A$.

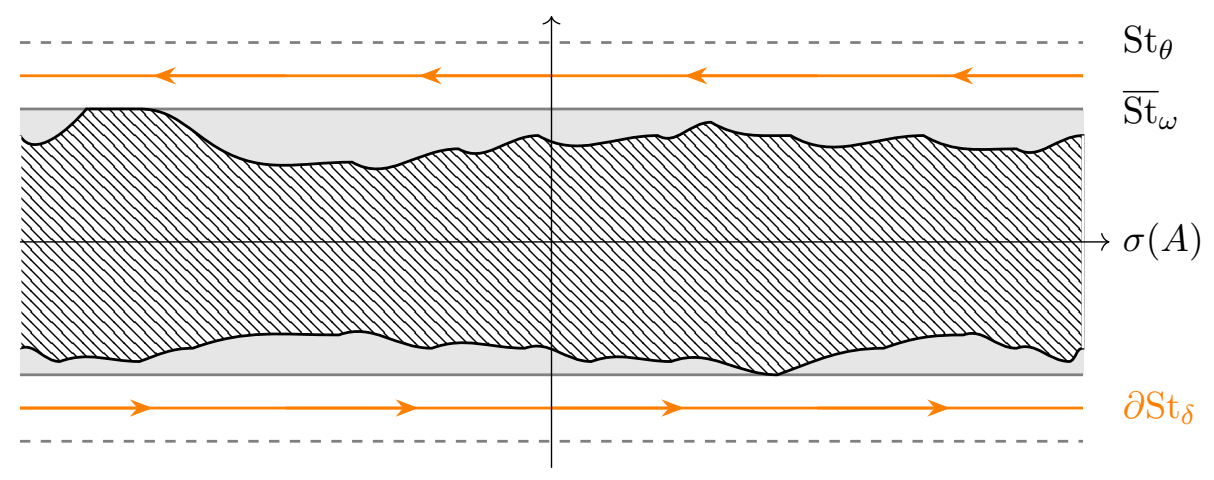

Figure 2.1: The spectrum of $A$ and the contour $\partial \mathrm{St}_{\delta}$.

\section{Remarks 2.3.5.}

1) As a consequence of Cauchy's integral theorem, the definition of the operator $\psi(A)$ does not depend on the particular choice of $\delta$. Hence, $\Phi_{\text {st }}$ is well defined.

2) The definition of $\mathcal{E}\left(\mathrm{St}_{\theta}\right)$ is motivated by the elementary calculus above, as it is a natural class of functions for which the Cauchy integrals in Definition 2.3.4 converge absolutely.

Lemma 2.3.6. In the situation of Definition 2.3.4 the following statements hold:

a) $\Phi_{\mathrm{st}}: \mathcal{E}\left[\mathrm{St}_{\omega}\right] \rightarrow \mathcal{L}(X)$ is an algebra homomorphism.

b) For each $|\operatorname{Im} \lambda|,|\operatorname{Im} \mu|>\omega$ one has

$$
\left(\frac{1}{(\lambda-\mathbf{z})(\mu-\mathbf{z})}\right)(A)=R(\lambda, A) R(\mu, A) .
$$

c) $\Phi_{\mathrm{st}}$ is non-degenerate.

Proof. Proving a) is a routine argument involving Cauchy's integral theorem and integral formula for holomorphic functions and the resolvent identity. The proof of b) can be found in [23, Proposition 4.2.1.b)], and c) is a consequence of b). 
Definition 2.3.7. Let $A$ be a strip type operator on $X$, and let $\omega:=\omega_{\mathrm{st}}(A)$. The algebraic extension of

$$
\Phi_{\mathrm{st}, A}: \mathcal{E}\left[\mathrm{St}_{\omega}\right] \rightarrow \mathcal{L}(X)
$$

with respect to the algebra $\mathrm{Hol}\left[\mathrm{St}_{\omega}\right]$ is called the (extended) holomorphic calculus of $A$. Whenever $f \in \operatorname{Hol}\left[\mathrm{St}_{\omega}\right]$ is anchored in $\mathcal{E}\left[\mathrm{St}_{\omega}\right]$ with respect to $\Phi_{\mathrm{st}, A}$, we write $f(A):=\Phi_{\mathrm{st}, A}(f)$.

Examples 2.3.8. Let $A$ and $\omega \geq 0$ be given as in Definition 2.3.7.

1) Each polynomial $p=\sum_{k=0}^{n} a_{k} \mathbf{z}^{k}$ is anchored in $\mathcal{E}\left[\mathrm{St}_{\omega}\right]$, and one has

$$
p(A)=\sum_{k=0}^{n} a_{k} A^{k} .
$$

In particular, $(\mathbf{z})(A)=A$.

2) Let $|\operatorname{Im} \lambda|>\omega$. Then, $(\lambda-\mathbf{z})^{-2} \in[f]_{\mathcal{E}}$ for each $f \in \mathrm{H}^{\infty}\left[\operatorname{St}_{\omega}\right]$. Hence, $f(A)$ is defined in the holomorphic calculus of $A$ for every function $f \in \mathrm{H}^{\infty}\left[\mathrm{St}_{\omega}\right]$, with

$$
f(A)=(\lambda-A)^{2}\left(\frac{f}{(\lambda-\mathbf{z})^{2}}\right)(A) .
$$

3) For each $|\operatorname{Im} \lambda|>\omega$ the function $(\lambda-\mathbf{z})^{-1}$ is anchored in $\mathcal{E}\left[\mathrm{St}_{\omega}\right]$ and one has

$$
\left(\frac{1}{\lambda-\mathbf{z}}\right)(A)=R(\lambda, A) \text {. }
$$

Remark 2.3.9. Let $f \in \mathrm{H}^{\infty}\left[\mathrm{St}_{\omega}\right]$, and let $|\operatorname{Im} \lambda|>\omega$. By Example 2.3.8.2) and Proposition 2.2.6.e),

$$
\operatorname{dom} A^{2}=\operatorname{ran} R(\lambda, A)^{2}=\operatorname{ran}\left(\frac{1}{(\lambda-\mathbf{z})^{2}}\right)(A) \subseteq \operatorname{dom} f(A) .
$$

Note that if $A$ is densely defined, then so is $A^{2}$ (see [4, p. 6]). Hence, whenever $A$ is densely defined, so is every operator $f(A)$.

The next theorem is an important tool for the abstract theory. (For a proof, see [23, Proposition 5.1.7.b)].)

Theorem 2.3.10 (Convergence lemma). Let $A$ be an operator of strip type $\omega \geq 0$ on a Banach space $X$. Let $\theta>\omega$, and let $\left(f_{n}\right)_{n}$ be a sequence in $\mathrm{H}^{\infty}\left(\mathrm{St}_{\theta}\right)$ satisfying

1) $f_{n} \rightarrow f$ pointwise on $\mathrm{St}_{\theta}$ for some function $f \in \mathrm{H}^{\infty}\left(\mathrm{St}_{\theta}\right)$;

2) $\sup _{n \in \mathbb{N}}\left\|f_{n}\right\|_{\infty, \mathrm{St}_{\theta}}<\infty$; and

3) $\sup _{n \in \mathbb{N}}\left\|f_{n}(A)\right\|<\infty$.

Then, $f_{n}(A) \rightarrow f(A)$ strongly on $\operatorname{dom} A^{2}$. In particular, if $A$ is densely defined, then $f(A) \in \mathcal{L}(X)$. 
Definition 2.3.11. Let $A$ be an operator of strip type $\omega \geq 0$ on $X$, and let $\theta>\omega$. We say that $A$ has a bounded $\mathrm{H}^{\infty}\left(\mathrm{St}_{\theta}\right)$-calculus if $f(A) \in \mathcal{L}(X)$ for every $f \in \mathrm{H}^{\infty}\left(\mathrm{St}_{\theta}\right)$.

Remarks 2.3.12. Let $A$ and $\theta$ be given as in the definition above.

1) If $A$ has a bounded $\mathrm{H}^{\infty}\left(\mathrm{St}_{\theta}\right)$-calculus, then the mapping $\mathrm{H}^{\infty}\left(\mathrm{St}_{\theta}\right) \rightarrow \mathcal{L}(X), f \mapsto$ $f(A)$, is a bounded algebra homomorphism. In particular, there is a constant $c \geq 0$ with

$$
\|f(A)\| \leq c\|f\|_{\infty, \mathrm{St}_{\theta}} \quad\left(f \in \mathrm{H}^{\infty}\left(\mathrm{St}_{\theta}\right) .\right.
$$

Proof. This is a consequence of the closed graph theorem. To see that the graph of the $\mathrm{H}^{\infty}\left(\mathrm{St}_{\theta}\right)$-calculus is closed, let $\left(f_{n}\right)_{n}$ be a sequence in $\mathrm{H}^{\infty}\left(\mathrm{St}_{\theta}\right)$ with $f_{n} \rightarrow 0$ uniformly on $\mathrm{St}_{\theta}$ and such that $f_{n}(A) \rightarrow T \in \mathcal{L}(X)$ in $X$. Fix $\psi \in \mathcal{E}\left(\mathrm{St}_{\theta}\right)$ and $\delta \in\left(\omega_{\text {st }}(A), \theta\right)$. By writing the operators $\left(\psi f_{n}\right)(A)$ as Cauchy integrals, we observe that

$$
\left\|\left(\psi f_{n}\right)(A)\right\| \lesssim_{A}\left\|\left(\psi f_{n}\right)(\mathrm{s}-\mathrm{i} \delta)\right\|_{1}+\left\|\left(\psi f_{n}\right)(\mathrm{s}+\mathrm{i} \delta)\right\|_{1} \stackrel{n \rightarrow \infty}{\longrightarrow} 0 .
$$

Hence,

$$
0=\lim _{n \rightarrow \infty}\left(\psi f_{n}\right)(A)=\lim _{n \rightarrow \infty} \psi(A) f_{n}(A)=\psi(A) T .
$$

As $\psi(A)$ is an injective operator if $\psi=(\lambda-\mathbf{z})^{-2}$ for $|\operatorname{Im} \lambda|>\theta$, it follows that $T=0$. Hence, the closed graph theorem is applicable.

2) One can show with Theorem 2.3.10 that if $A$ is densely defined, then $A$ has a bounded $\mathrm{H}^{\infty}\left(\mathrm{St}_{\theta}\right)$-calculus if and only if there is a constant $c \geq 0$ such that $\|\psi(A)\| \leq c\|\psi\|_{\infty, \mathrm{St}_{\omega}}$ for all $\psi \in \mathrm{H}_{0}^{\infty}\left(\mathrm{St}_{\omega}\right)$. (See also [10, Corollary 2.2].) Later, when discussing the Hörmander calculus, we prove a similar result, see Theorem 4.3 .3 .

We close this section with a discussion on particular anchor sets which play a key role in Chapter 4

Lemma 2.3.13. Let $A$ be an operator of strip type $\omega \geq 0$ on a Banach space $X$, let $\theta>\omega$, and let $0 \neq \psi \in \mathrm{H}_{0}^{\infty}\left(\mathrm{St}_{\theta}\right)$. Then the set

$$
\mathcal{E}_{\psi}:=\left\{\tau_{t} \psi \mid t \in \mathbb{R}\right\}
$$

is an anchor set with respect to $\Phi_{\mathrm{st}, A}$. Moreover, if $A$ is densely defined, then $\mathcal{E}_{\psi}$ has total range (see Definition 2.2.7).

Proof. Without loss of generality we may assume that

$$
\left\|\psi_{0}\right\|_{2}^{2}=\int_{\mathbb{R}} \psi^{*}(t) \psi(t) \mathrm{d} t=\int_{\mathbb{R}} \psi^{*}(s-t) \psi(s-t) \mathrm{d} t=1 \quad(s \in \mathbb{R}) .
$$

Then, by the identity theorem for holomorphic functions,

$$
\int_{\mathbb{R}} \psi^{*}(z-t) \psi(z-t) \mathrm{d} t=1
$$


for all $z \in \mathrm{St}_{\theta}$. Set

$$
X_{\psi}:=\bigcup_{t \in \mathbb{R}} \operatorname{ran}\left(\tau_{t} \psi\right)(A),
$$

and let $|\operatorname{Im} \lambda|>\omega$. Then, for a suitable $\delta>0$,

$$
\begin{aligned}
R(\lambda, A)^{2} & =\frac{1}{2 \pi \mathrm{i}} \int_{\partial \mathrm{St}_{\delta}} \frac{1}{(\lambda-z)^{2}} R(z, A) \mathrm{d} z \\
& =\frac{1}{2 \pi \mathrm{i}} \int_{\partial \mathrm{St}_{\delta}}\left(\int_{\mathbb{R}} \frac{\psi^{*}(z-t) \psi(z-t)}{(\lambda-z)^{2}} \mathrm{~d} t\right) R(z, A) \mathrm{d} z \\
& =\int_{\mathbb{R}}\left(\frac{1}{2 \pi \mathrm{i}} \int_{\partial \mathrm{St}_{\delta}} \frac{\psi^{*}(z-t) \psi(z-t)}{(\lambda-z)^{2}} R(z, A) \mathrm{d} z\right) \mathrm{d} t \\
& =\int_{\mathbb{R}}\left(\tau_{t} \psi^{*}\right)(A)\left(\tau_{t} \psi\right)(A) R(\lambda, A)^{2} \mathrm{~d} t .
\end{aligned}
$$

Here we have used identity 2.3.3 in the second line and Fubini's theorem in the third line. It follows that

$$
\operatorname{ran} R(\lambda, A)^{2}=\operatorname{dom} A^{2} \subseteq \overline{\operatorname{lin} X_{\psi}},
$$

and

$$
\bigcap_{t \in \mathbb{R}} \operatorname{ker}\left(\tau_{t} \psi\right)(A) \subseteq \operatorname{ker} R(\lambda, A)^{2}=\{0\}
$$

This yields the claim.

As a corollary we obtain

Corollary 2.3.14. Let $A$ be an operator of strip type $\omega \geq 0$ on a Banach space $X$, and let $\theta>\omega$. Then the operator $\psi(A)=\mathrm{e}^{-A^{2}}$ is injective.

Proof. First note that

$$
\tau_{t} \psi=\mathrm{e}^{-(\mathbf{z}-t)^{2}}=\mathrm{e}^{2 t \mathbf{z}-t^{2}-\mathbf{z}^{2}}=f_{t} \cdot \psi
$$

for every $t \in \mathbb{R}$, where $f_{t}:=\mathrm{e}^{2 t \mathbf{z}-t^{2}}$. Each function $f_{t}$ is anchored in the holomorphic calculus of $A$ (see [23, p. 96]). Hence, whenever $x \in \operatorname{ker} \psi(A)$,

$$
\left(\tau_{t} \psi\right)(A) x=\left(f_{t} \cdot \psi\right)(A) x=f_{t}(A) \psi(A) x=0
$$

for each $t \in \mathbb{R}$. As $\left\{\tau_{t} \psi \mid t \in \mathbb{R}\right\}$ is an anchor set by Lemma 2.3.13, $x=0$. Consequently, $\psi(A)$ is injective.

\subsection{The Holomorphic Calculus for Injective Sectorial Op- erators}

We also recall all relevant definitions and results for the classical holomorphic calculus of (injective) sectorial operators. Throughout, let $X$ be a complex Banach space.

We start with the definition of a sectorial operator. 
Definition 2.4.1. A possibly unbounded operator $A \subseteq X \oplus X$ is called sectorial if there is $\omega \in[0, \pi)$ with

$$
\sigma(A) \subseteq \overline{\mathrm{S}}_{\omega} \quad \text { and } \quad \sup _{\lambda \in \mathbb{C} \backslash \overline{\mathrm{S}}_{\theta}}\|\lambda R(\lambda, A)\|<\infty \quad \text { for all } \quad \theta \in(\omega, \pi)
$$

For a sectorial operator, the angle

$$
\omega_{\text {se }}(A):=\inf \{\omega \in[0, \pi) \mid 2.4 .1 \text { holds }\}
$$

is called the angle of sectoriality of $A$. If $\omega_{\mathrm{se}}(A)=0$, then $A$ is also said to be 0 -sectorial.

For the sectorial case, the following function spaces are of interest.

Definition 2.4.2. Let $\omega \in(0, \pi)$. The space

$$
\mathcal{E}\left(\mathrm{S}_{\omega}\right):=\left\{\psi \in \mathrm{H}^{\infty}\left(\mathrm{S}_{\omega}\right) \mid \forall r \in(-\omega, \omega): \quad \psi\left(\mathrm{e}^{\mathrm{i} r} \mathrm{~s}\right) \in \mathrm{L}^{1}\left((0, \infty), \frac{\mathrm{d} s}{s}\right)\right\}
$$

is called the algebra of elementary functions on $S_{\omega}$. We further set

$$
\mathrm{H}_{0}^{\infty}\left(\mathrm{S}_{\omega}\right):=\left\{\psi \in \mathrm{H}^{\infty}\left(\mathrm{S}_{\omega}\right)\left|\exists c_{0}, c_{1}>0: \quad\right| \psi \mid \leq c_{0} \min \left\{|\mathbf{z}|^{c_{1}},|\mathbf{z}|^{-c_{1}}\right\}\right\} .
$$

For $\mathrm{F} \in\left\{\mathcal{E}, \mathrm{H}_{0}^{\infty}, \mathrm{H}^{\infty}, \mathrm{Hol}\right\}$ and $\omega^{\prime} \in[0, \pi)$ we write

$$
\mathrm{F}\left[\mathrm{S}_{\omega^{\prime}}\right]:=\bigcup_{\omega^{\prime}<\omega<\pi} \mathrm{F}\left(\mathrm{S}_{\omega}\right)
$$

Remark 2.4.3. Note that for each $\omega \in(0, \pi)$ and $\mathrm{F} \in\left\{\mathcal{E}, \mathrm{H}_{0}^{\infty}, \mathrm{H}^{\infty}, \mathrm{Hol}\right\}$ one has that $\psi \in \mathrm{F}\left(\mathrm{S}_{\omega}\right)$ if and only if $\psi \circ \mathrm{e}^{\mathbf{z}} \in \mathrm{F}\left(\mathrm{St}_{\omega}\right)$.

The elementary holomorphic calculus of a sectorial operator is defined as follows.

Definition 2.4.4. Let $A$ be a sectorial operator on $X$, and let $\omega:=\omega_{\mathrm{se}}(A)$. For $\omega<$ $\delta<\theta<\pi$ and $\psi \in \mathcal{E}\left(\mathrm{S}_{\theta}\right)$ define

$$
\begin{aligned}
\psi(A) & :=\frac{1}{2 \pi \mathrm{i}} \int_{\partial \mathrm{S}_{\delta}} \psi(z) R(z, A) \mathrm{d} z \\
& =\frac{1}{2 \pi \mathrm{i}}\left(\int_{0}^{\infty} \mathrm{e}^{-\mathrm{i} \delta} \psi\left(\mathrm{e}^{-\mathrm{i} \delta} s\right) R\left(\mathrm{e}^{-\mathrm{i} \delta} s, A\right) \mathrm{d} s-\int_{0}^{\infty} \mathrm{e}^{\mathrm{i} \delta} \psi\left(\mathrm{e}^{\mathrm{i} \delta} s\right) R\left(\mathrm{e}^{\mathrm{i} \delta} s, A\right) \mathrm{d} s\right) .
\end{aligned}
$$

The mapping

$$
\Phi_{\mathrm{se}}:=\Phi_{\mathrm{se}, A}: \mathcal{E}\left[\mathrm{S}_{\omega}\right] \rightarrow \mathcal{L}(X), \quad \Phi_{\mathrm{se}}(\psi):=\psi(A)
$$

is called the elementary calculus of $A$.

Remark 2.4.5. As in the strip case, the definition of the operator $\psi(A)$ does not depend on the particular choice of $\delta$, whence $\Phi_{\text {se }}$ is well defined. 


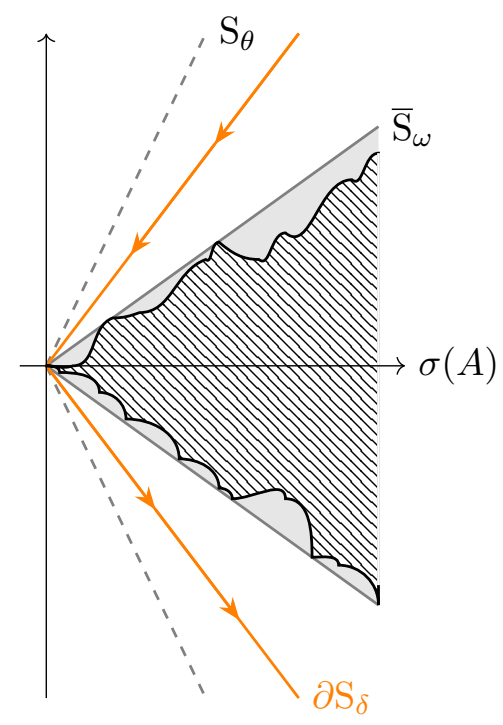

Figure 2.2: The spectrum of $A$ and the path of integration along $\partial \mathrm{S}_{\delta}$.

Lemma 2.4.6. In the situation of Definition 2.4.4 the following statements hold:

a) $\Phi_{\mathrm{se}}: \mathcal{E}\left[\mathrm{S}_{\omega}\right] \rightarrow \mathcal{L}(X)$ is an algebra homomorphism.

b) For each $|\arg \lambda|,|\arg \mu|>\omega$ one has

$$
\left(\frac{\mathbf{z}}{(\lambda-\mathbf{z})(\mu-\mathbf{z})}\right)(A)=A R(\lambda, A) R(\mu, A) .
$$

c) $\Phi_{\mathrm{se}}$ is non-degenerate if and only if $A$ is injective.

Proof. As in the strip case, a) is routine. See also [22, Proposition 2.2.a)]. For a proof of b), see [22, Proposition 2.2.d)]. Statement c) can be concluded from [23, Theorem 2.3.3.c)].

For an injective sectorial operator, the terms extended holomorphic calculus and bounded $\mathrm{H}^{\infty}$-calculus are defined as in the strip case but with the obvious changes.

Examples 2.4.7. Let $A$ be an injective sectorial operator, and let $\omega=\omega_{\mathrm{se}}(A)$.

1) As in the strip case, each polynomial and each function $f \in \mathrm{H}^{\infty}\left[\mathrm{S}_{\omega}\right]$ is anchored in the sectorial calculus, and one has $(\mathbf{z})(A)=A$.

2) The resolvent of $A$ can be recovered in the sense that if $|\arg \lambda|>\omega$, then the function $(\lambda-\mathbf{z})^{-1}$ is anchored in the sectorial calculus and one has

$$
\left(\frac{1}{\lambda-\mathbf{z}}\right)(A)=R(\lambda, A)
$$


3) The operator $\log A$ is well defined within the holomorphic calculus of $A$. Indeed, just note for example that

$$
\log \mathbf{z} \cdot \frac{\mathbf{z}^{2}}{(1+\mathbf{z})^{4}} \in \mathcal{E}\left[\mathrm{S}_{\omega}\right] .
$$

There is a strong connection between the holomorphic calculus of sectorial operators and strip type operators. This is expressed in the next two theorems. First however, we recall the so-called (BIP) property.

Definition 2.4.8. Let $A$ be an injective sectorial operator on $X$. The operator $A$ is said to have bounded imaginary powers or property (BIP) if $A^{-\mathrm{i} s}:=\left(\mathbf{z}^{-\mathrm{i} s}\right)(A) \in \mathcal{L}(X)$ for each $s \in \mathbb{R}$ and $\left(A^{-\mathrm{i} s}\right)_{s \in \mathbb{R}}$ is a $C_{0}$-group (see Definition 2.5.3).

Theorem 2.4.9. Let $A$ be an injective sectorial operator. Then the following statements hold:

a) The operator $\log A$ (defined within the holomorphic calculus of $A$ ) is a strip type operator of strip type

$$
\omega_{\mathrm{st}}(\log A)=\omega_{\mathrm{se}}(A) .
$$

b) The operator $A$ has the (BIP) property if and only if -i $\log A$ generates a $C_{0}$-group. In this case, $-\mathrm{i} \log A$ is the generator of the $C_{0}$-group $\left(A^{-\mathrm{i} s}\right)_{s \in \mathbb{R}}$.

Proof. For a), see [23, Theorem 4.3.1], and for b), see [23, Corollary 3.5.7].

The next theorem essentially follows from [23, Corollary 4.2.5].

Theorem 2.4.10 (Composition rule). Let $A$ be an injective sectorial operator, let $\omega \in$ $\left(\omega_{\mathrm{se}}(A), \pi\right)$, and let $f: \mathrm{St}_{\omega} \rightarrow \mathbb{C}$ be a holomorphic function. Then the operator $f(\log A)$ is defined within the holomorphic calculus of $\log A$ if and only if the operator $(f \circ \log )(A)$ is defined within the holomorphic calculus of $A$. In this case,

$$
f(\log A)=(f \circ \log )(A) .
$$

\subsection{Groups of Operators and their Functional Calculus}

Here we briefly discuss $C_{0}$-groups together with the Fourier-Stieltjes calculus and its connection to the holomorphic calculus of strip type operators. Throughout, let $X$ denote a complex Banach space.

\section{$C_{0}$-Groups}

$C_{0}$-groups are closely connected with $C_{0}$-semigroups.

Definition 2.5.1. A family $\left(T_{t}\right)_{t \geq 0}$ of bounded operators on $X$ is called $C_{0}$-semigroup if the following conditions are satisfied:

1) $\forall s, t \geq 0: \quad T_{s+t}=T_{s} T_{t}$; 
2) $\forall x \in X: \quad \lim _{t \searrow 0} T_{t} x=x$.

A family $\left(U_{s}\right)_{s \in \mathbb{R}}$ is called $C_{0}$-group, if

1) $\left(U_{t}\right)_{t \geq 0}$ is a $C_{0}$-semigroup;

2) $\forall s \in \mathbb{R}: \quad U_{-s}=U_{s}^{-1}$.

Remark 2.5.2. If $\left(T_{t}\right)_{t \geq 0}$ is a $C_{0}$-semigroup, then $T_{0}=\mathrm{I}$. Indeed, for each $x \in X$ one has

$$
x=\lim _{t \searrow 0} T_{t} x=\lim _{t \searrow 0} T_{0} T_{t} x=T_{0} x .
$$

The group type of a $C_{0}$-group describes the essential growth behavior of the group.

Definition 2.5.3. Let $U=\left(U_{s}\right)_{s \in \mathbb{R}}$ be a $C_{0}$-group. The group type of $\left(U_{s}\right)_{s \in \mathbb{R}}$ is defined as

$$
\theta(U):=\inf \left\{\theta \geq 0 \mid \sup _{s \in \mathbb{R}}\left(\mathrm{e}^{-\theta|s|}\left\|U_{s}\right\|\right)<\infty\right\} .
$$

Remark 2.5.4. The group type of a $C_{0}$-group is always finite. This is a simple consequence of [13, Proposition 5.5, p. 39].

To each $C_{0}$-semigroup one associates a (possibly unbounded) operator $B$, the socalled generator.

Definition 2.5.5. Let $\left(T_{t}\right)_{t \geq 0}$ be a $C_{0}$-semigroup on $X$. Then the operator

$$
B:=\left\{(x, y) \in X \oplus X \mid y=\lim _{t \searrow 0} \frac{1}{t}\left(T_{t} x-x\right)\right\}
$$

is called the generator of $\left(T_{t}\right)_{t \geq 0}$, and one says that $B$ generates the semigroup $\left(T_{t}\right)_{t \geq 0}$. If $\left(U_{s}\right)_{s \in \mathbb{R}}$ is a $C_{0}$-group, the generator of $\left(U_{t}\right)_{t \geq 0}$ is also called the generator of $\left(U_{s}\right)_{s \in \mathbb{R}}$.

The following result can be found in each introductory text for operator semigroups, see [13, Theorem 1.4, p. 51] for example.

Proposition 2.5.6. Let $B$ be the generator of a $C_{0}$-semigroup $\left(T_{t}\right)_{t \geq 0}$ on $X$. Then $B$ is closed and densely defined, and the semigroup $\left(T_{t}\right)_{t \geq 0}$ is uniquely determined by $B$.

The following theorem, which goes back to a famous result by Hille and Yosida, characterizes generators of $C_{0}$-semigroups (see [13, Theorem 3.8 on p. 77]).

Theorem 2.5.7 (Feller, Miyadera, Phillips, 1952). Let $B$ be a densely defined operator on a Banach space $X$, let $\theta \in \mathbb{R}$, and let $M \geq 1$. Then the following statements are equivalent:

(i) $B$ generates a $C_{0}$-semigroup $\left(T_{t}\right)_{t \geq 0}$ on $X$ and one has

$$
\left\|T_{t}\right\| \leq M \mathrm{e}^{\theta t} \quad(t \geq 0) .
$$

(ii) One has $\{\lambda \in \mathbb{C} \mid \operatorname{Re} \lambda>\theta\} \subseteq \rho(B)$ and

$$
\left\|R(\lambda, B)^{n}\right\| \leq \frac{M}{(\theta-\operatorname{Re} \lambda)^{n}} \quad(n \in \mathbb{N}, \operatorname{Re} \lambda>\theta) .
$$




\section{Remarks 2.5.8.}

There is a strong connection between group generators and strip type operators:

1) Let $B$ be the generator of a $C_{0}$-group $\left(U_{s}\right)_{s \in \mathbb{R}}$. Then it is easy to see that $\left(U_{-t}\right)_{t \geq 0}$ is a $C_{0}$-semigroup with generator $-B$. Hence, if one writes $-\mathrm{i} A=B$, then Theorem 2.5 .7 implies that $A$ is a strip type operator with $\omega_{\text {st }}(A) \leq \theta(U)$. If $X$ is a Hilbert space, one can even show that $\omega_{\text {st }}(A)=\theta(U)$ (see [24, Corollary 11.6]). However, equality does not hold in general. See [23, p. 102 - 103] for a counterexample.

2) Suppose that $-\mathrm{i} A$ generates a $C_{0}$-group $\left(U_{s}\right)_{s \in \mathbb{R}}$. Then one can show that

$$
U_{s}=\left(\mathrm{e}^{-\mathrm{i} s \mathbf{z}}\right)(A)
$$

for all $s \in \mathbb{R}$, where the operator on the right hand side is defined in the holomorphic calculus of $A$.

3 ) If $A$ is a strip type operator, then $-\mathrm{i} A$ need not be the generator of a $C_{0}$-group. In fact, $-\mathrm{i} A$ generates a $C_{0}$-group if and only if $A$ is densely defined and of so-called strong strip type. (For the definition see [23, p. 92] for example.)

4) Let $A$ be a strip type operator with a bounded $\mathrm{H}^{\infty}\left(\mathrm{St}_{\theta}\right)$-calculus for some width $\theta>0$. For $s \in \mathbb{R}$ define

$$
U_{s}:=\left(\mathrm{e}^{-\mathrm{i} s \mathbf{z}}\right)(A) .
$$

Then, by appealing to Theorem 2.3.10, one can show that $\left(U_{s}\right)_{s \in \mathbb{R}}$ is a $C_{0}$-group. The generator of this group is $-\mathrm{i} A$. As a consequence of the bounded $\mathrm{H}^{\infty}\left(\mathrm{St}_{\theta}\right)$ calculus, one has $\theta(U) \leq \theta$ since $\left\|\mathrm{e}^{-\mathrm{i} s \mathbf{z}}\right\|_{\infty, \mathrm{St}_{\theta}}=\mathrm{e}^{\theta|s|}$ for each $s \in \mathbb{R}$.

5) Suppose that $-\mathrm{i} A$ generates a $C_{0}$-group $\left(U_{s}\right)_{s \in \mathbb{R}}$. By the remark above, the natural threshold for $\theta$ in the question whether $A$ has a bounded $\mathrm{H}^{\infty}\left(\mathrm{St}_{\theta}\right)$-calculus is $\theta(U)$ rather than $\omega_{\mathrm{st}}(A)$.

\section{The Fourier-Stieltjes Calculus}

The Fourier-Stieltjes calculus is a functional calculus tailor made for strongly continuous groups of operators. This calculus is defined on an algebra of measures.

Definition 2.5.9. Let $M(\mathbb{R})$ denote the space of all (regular) complex Borel measures on $\mathbb{R}$ (see [46, Chapter 6]), and equip this space with the total variation norm

$$
\|\mu\|_{\mathrm{M}(\mathbb{R})}:=|\mu|(\mathbb{R}) \quad(\mu \in \mathrm{M}(\mathbb{R})) .
$$

The convolution of two measures $\mu, \nu \in \mathrm{M}(\mathbb{R})$ is defined as the (uniquely determined) measure $\mu * \nu \in \mathrm{M}(\mathbb{R})$ satisfying

$$
\int_{\mathbb{R}} f(s) \mathrm{d}(\mu * \nu)(s)=\int_{\mathbb{R}} \int_{\mathbb{R}} f(s+t) \mathrm{d} \nu(s) \mathrm{d} \mu(t) \quad\left(f \in \mathrm{C}_{0}(\mathbb{R})\right) .
$$

For $\omega>0$ set

$$
\mathrm{M}_{\omega}(\mathbb{R}):=\left\{\mu \in \mathrm{M}(\mathbb{R})\left|\int_{\mathbb{R}} \mathrm{e}^{\omega|s|} \mathrm{d}\right| \mu \mid(s)<\infty\right\}
$$


and equip this space with the norm

$$
\|\mu\|_{\mathrm{M}_{\omega}(\mathbb{R})}:=\int_{\mathbb{R}} \mathrm{e}^{\omega|s|} \mathrm{d}|\mu|(s) \quad\left(\mu \in \mathrm{M}_{\omega}(\mathbb{R})\right) .
$$

For reasons of convenience we also write $\mathrm{M}_{0}(\mathbb{R}):=\mathrm{M}(\mathbb{R})$.

Proposition 2.5.10. For each $\omega \geq 0$ the space $\left(\mathrm{M}_{\omega}(\mathbb{R}),\|\cdot\|_{\mathrm{M}_{\omega}(\mathbb{R})}\right)$ is a unital Banach algebra with respect to convolution of measures.

The proof is straightforward and therefore omitted.

Definition 2.5.11. Let $-\mathrm{i} A$ be the generator of a $C_{0}$-group $\left(U_{s}\right)_{s \in \mathbb{R}}$ on $X$. Let $\omega \geq 0$ such that

$$
\sup _{s \in \mathbb{R}}\left(\mathrm{e}^{-\omega|\mathbf{s}|}\left\|U_{s}\right\|\right)<\infty .
$$

For $\mu \in \mathrm{M}_{\omega}(\mathbb{R})$ define

$$
U_{\mu}:=\int_{\mathbb{R}} U_{s} \mathrm{~d} \mu(s),
$$

where the integral is taken in the strong sense. In the case that $\mu=f \mathrm{~d} s$ for some function $f \in \mathrm{L}^{1}\left(\mathbb{R}, \mathrm{e}^{\omega|s|} \mathrm{d} s\right)$, one also writes $U_{f}:=U_{f} \mathrm{~d} s$. The mapping

$$
\mathrm{M}_{\omega}(\mathbb{R}) \rightarrow \mathcal{L}(X), \quad \mu \mapsto U_{\mu}
$$

is called the Fourier-Stieltjes calculus of $A$, sometimes the Fourier-Stieltjes calculus of $\left(U_{s}\right)_{s \in \mathbb{R}}$.

Proposition 2.5.12. In the situation of Definition 2.5.11, the mapping

$$
\mathrm{M}_{\omega}(\mathbb{R}) \rightarrow \mathcal{L}(X), \quad \mu \mapsto U_{\mu}
$$

is a bounded, unital algebra homomorphism.

The proof is a straightforward adaption of the proof of [24, Lemma 5.3 and Theorem 5.4.d)] and thus omitted.

The next two propositions establish a strong connection between the holomorphic calculus of $A$ and the Fourier-Stieltjes calculus of $\left(U_{s}\right)_{s \in \mathbb{R}}$. For proofs, see [24, Theorem 11.15.2)] and [24, Theorem 11.15.4)].

Proposition 2.5.13. For each $\omega \geq 0$ one has $\mathcal{F}^{-1}\left(\mathcal{E}\left[\mathrm{St}_{\omega}\right]\right) \subseteq \mathrm{L}^{1}\left(\mathbb{R}, \mathrm{e}^{\omega|s|} \mathrm{d} s\right)$.

Proposition 2.5.14. In the situation of Definition 2.5.11, the Fourier-Stieltjes calculus extends the elementary holomorphic calculus of $A$ in the sense that

$$
U_{\psi^{\vee}}=\psi(A)
$$

for each $\psi \in \mathcal{E}\left[\mathrm{St}_{\omega}\right]$. 


\subsection{Geometry of Banach Spaces}

In this section we gather all notions from the geometry of Banach spaces needed for the discussions in Section 4.3, and to a lesser extent in Section 4.4 and Chapter 5.

Throughout, let $(\Omega, \mathbb{P})$ be a probability space and let $X$ be a Banach space.

Definition 2.6.1. A random variable $r: \Omega \rightarrow\{-1,1\}$ is called a Rademacher variable or a Rademacher if $r$ is $\left(\frac{1}{2}, \frac{1}{2}\right)$-Bernoulli distributed, i.e., $\mathbb{P}(r=-1)=\mathbb{P}(r=$ $1)=\frac{1}{2}$. A random variable $\gamma: \Omega \rightarrow \mathbb{R}$ is called a standard Gaussian variable or just standard Gaussian, if $\gamma$ is normally distributed with mean 0 and variance 1. For a map $f: \Omega \rightarrow \mathbb{C}$ and $x \in X$ we write $f x: \Omega \rightarrow X, s \mapsto f(s) x$.

The four notions of Banach space geometry that we are interested in are: type, cotype, Pisier's property $(\alpha)$, and $\gamma$-boundedness.

Definition 2.6.2. The space $X$ has (Rademacher) type $p \in[1,2]$ if there is a constant $c=c(X, p) \geq 0$ such that

$$
\left(\mathbb{E}\left\|\sum_{k=1}^{n} r_{k} x_{k}\right\|_{X}^{2}\right)^{\frac{1}{2}} \leq c\left(\sum_{k=1}^{n}\left\|x_{k}\right\|_{X}^{p}\right)^{\frac{1}{p}}
$$

for all $n \in \mathbb{N}, x_{1}, \ldots, x_{n} \in X$ and independent Rademachers $r_{1}, \ldots, r_{n}$. We say that $X$ is of (Rademacher) cotype $q \in[2, \infty)$ if there is a constant $c=c(X, q) \geq 0$ such that

$$
\left(\sum_{k=1}^{n}\left\|x_{k}\right\|_{X}^{q}\right)^{\frac{1}{q}} \leq c\left(\mathbb{E}\left\|\sum_{k=1}^{n} r_{k} x_{k}\right\|_{X}^{2}\right)^{\frac{1}{2}}
$$

for all $n \in \mathbb{N}, x_{1}, \ldots, x_{n} \in X$ and all independent Rademachers $r_{1}, \ldots, r_{n}$. Cotype $\infty$ is defined with the obvious changes in the inequality above. The space $X$ is said to be of finite cotype if it has cotype $q<\infty$.

Definition 2.6.3. The Banach space $X$ has Pisier's property $(\alpha)$, if for each finite array $\left(x_{n, k}\right)_{1 \leq n \leq N, 1 \leq k \leq K}$ in $X$ and independent families of Gaussians $\left(\gamma_{n}\right)_{1 \leq n \leq N}$, $\left(\gamma_{k}^{\prime}\right)_{1 \leq k \leq K}$ and $\left(\gamma_{n, k}\right)_{1 \leq n \leq N, 1 \leq k \leq K}$

$$
\mathbb{E} \mathbb{E}^{\prime}\left\|\sum_{n=1}^{N} \sum_{k=1}^{K} \gamma_{n} \gamma_{k}^{\prime} x_{n, k}\right\|_{X}^{2} \bar{\sim}_{X} \mathbb{E}\left\|\sum_{n=1}^{N} \sum_{k=1}^{K} \gamma_{n, k} x_{n, k}\right\|_{X}^{2} .
$$

\section{Remarks 2.6.4.}

1) Pisier's property $(\alpha)$ is also known in the literature as Pisier's contraction property, see [31, Definition 7.5.1] and [31, Corollary 7.5.19].

2) It is obvious from the definitions above that type, cotype, and the property $(\alpha)$ are inherited by closed subspaces of $X$. 
3) For every Hilbert space $H$ one has type $(H)=\operatorname{cotype}(H)=2$ ([31, Example 7.1.2]). Moreover, every Hilbert space has property $(\alpha)$ ([31, Example 7.5.2]).

4) Next to Hilbert spaces, scalar $\mathrm{L}^{p}$-spaces also have good geometric properties: Let $Y:=\mathrm{L}^{p}\left(\Omega^{\prime}, \mu\right)$ for some measure space $\left(\Omega^{\prime}, \mu\right)$ and $1 \leq p<\infty$. Then,

- $Y$ has Pisier's property $(\alpha)$ ([31, Proposition 7.5.3]),

- $Y$ has type $\min \{2, p\}$ ([31, Proposition 7.1.4.(1)]), and

- $Y$ has cotype $\max \{2, p\}$ ([31, Proposition 7.1.4.(2)]). In particular, $Y$ is of finite cotype.

Definition 2.6.5. A subset $\mathcal{T} \subseteq \mathcal{L}(X)$ is called $\gamma$-bounded if there is a constant $c \geq 0$ such that

$$
\left(\mathbb{E}\left\|\sum_{k=1}^{n} \gamma_{k} T_{k} x_{k}\right\|_{X}^{2}\right)^{\frac{1}{2}} \leq c\left(\mathbb{E}\left\|\sum_{k=1}^{n} \gamma_{k} x_{k}\right\|_{X}^{2}\right)^{\frac{1}{2}}
$$

for each $n \in \mathbb{N}, T_{1}, \ldots, T_{n} \in \mathcal{T}, x_{1}, \ldots, x_{n} \in X$, and independent Gaussians $\gamma_{1}, \ldots, \gamma_{n}$. The smallest admissible constant $c$ in the inequality above is denoted by $\llbracket \mathcal{T} \rrbracket_{\gamma}$ and is called the $\gamma$-bound of $\mathcal{T}$.

We note the following two results for reference. Both statements show how $\gamma$-bounded sets can be obtained in certain situations. For the first theorem see [31, Proposition 8.1.22].

Theorem 2.6.6. Let $X$ be a Banach space, and let $\mathcal{T} \subseteq \mathcal{L}(X)$ be a $\gamma$-bounded set. Then both the closure $\overline{\mathcal{T}}^{\text {st }}$ in the strong operator topology and the closure $\overline{\mathcal{T}}^{\text {we }}$ in the weak operator topology are $\gamma$-bounded with

$$
\llbracket \overline{\mathcal{T}}^{\mathrm{st}} \rrbracket_{\gamma}=\llbracket \overline{\mathcal{T}}^{\mathrm{we}} \rrbracket_{\gamma}=\llbracket \mathcal{T} \rrbracket_{\gamma} .
$$

For the next statement see [31, Theorem 8.5.12] and [31, Remark 8.5.13].

Theorem 2.6.7. Let $X$ be a Banach space of type $p \in[1,2]$ and cotype $q \in[2, \infty]$, and let $(\Omega, \mu)$ be a measure space. Let $r \in[1, \infty)$ with $\frac{1}{r}>\frac{1}{p}-\frac{1}{q}$, and let $F: \Omega \rightarrow \mathcal{L}(X)$ such that $F(\mathbf{s}) x \in \mathrm{L}^{r}(\Omega, \mu ; X)$ for all $x \in X$ and

$$
\sup _{\|x\| \leq 1}\|F(\mathbf{s}) x\|_{r}<\infty .
$$

Consider for each function $\varphi \in \mathrm{L}^{r^{\prime}}(\Omega, \mu)$ the operator

$$
I_{F, \varphi}: X \rightarrow X, \quad x \mapsto \int_{\Omega} \varphi(s) \cdot F(s) x \mathrm{~d} \mu(s) .
$$

Then the set $\mathcal{T}_{F, r^{\prime}}:=\left\{I_{F, \varphi} \mid\|\varphi\|_{r^{\prime}} \leq 1\right\}$ is $\gamma$-bounded with

$$
\llbracket \mathcal{T}_{F, r^{\prime}} \rrbracket_{\gamma} \lesssim X \sup _{\|x\| \leq 1}\|F(\mathbf{s}) x\|_{r}
$$


Definition 2.6.8. An algebra homomorphism $\Phi: \mathcal{A} \rightarrow \mathcal{L}(X)$, where $\mathcal{A}$ is some normed algebra, is called $\gamma$-bounded if the set

$$
\left\{\Phi(a) \mid\|a\|_{\mathcal{A}} \leq 1\right\}
$$

is a $\gamma$-bounded subset of $\mathcal{L}(X)$.

\section{Remarks 2.6.9.}

1) Replacing Gaussian variables with Rademachers in Definition 2.6.5 and Definition 2.6 .8 , respectively, yields the notion of $R$-bounded sets and $R$-bounded algebra homomorphisms.

2) Both [31, Proposition 8.1.22] and [31, Theorem 8.5.12, resp. Remark 8.5.13] are formulated for $R$-boundedness instead of $\gamma$-boundedness. The proofs however work exactly the same for $\gamma$-boundedness.

3) In general, we prefer to work with $\gamma$-boundedness and standard Gaussians instead of $R$-boundedness and Rademachers. Note however, that if $X$ is of finite cotype, the notions of $\gamma$-boundedness and $R$-boundedness coincide ([31, Theorem 8.1.3.(2)]). Moreover, every Banach space with Pisier's property $(\alpha)$ necessarily has finite cotype ([31, Corollary 7.5.13]).

The next theorem is a direct corollary of [31, Theorem 6.1.13.(ii)] and shows that for every Banach space $X$ the homomorphism

$$
\mathbb{C} \rightarrow \mathcal{L}(X), \quad \alpha \mapsto \alpha \mathrm{I},
$$

is $\gamma$-bounded.

Theorem 2.6.10 (Kahane's Contraction Principle). Let $X$ be a Banach space. Then, for each $n \in \mathbb{N}, \alpha_{1}, \ldots, \alpha_{n} \in \mathbb{C}, x_{1}, \ldots, x_{n}$, and all independent Gaussians $\gamma_{1}, \ldots, \gamma_{n}$,

$$
\left(\mathbb{E}\left\|\sum_{k=1}^{n} \gamma_{k} \alpha_{k} x_{k}\right\|_{X}^{2}\right)^{\frac{1}{2}} \leq \frac{\pi}{2} \cdot \max _{1 \leq j \leq n}\left|\alpha_{j}\right| \cdot\left(\mathbb{E}\left\|\sum_{k=1}^{n} \gamma_{k} x_{k}\right\|_{X}^{2}\right)^{\frac{1}{2}}
$$

We close with the following simple observation.

Proposition 2.6.11. Let $X_{0}, X_{1}$ be Banach spaces, let $\mathcal{A}$ be a normed algebra, and let $\Phi_{0}: \mathcal{A} \rightarrow \mathcal{L}\left(X_{0}\right)$ and $\Phi_{1}: \mathcal{A} \rightarrow \mathcal{L}\left(X_{1}\right)$ be algebra homomorphisms. Consider the induced algebra homomorphism

$$
\Phi: \mathcal{A} \rightarrow \mathcal{L}\left(X_{0} \oplus X_{1}\right), \quad \Phi(a)\left(x_{0}, x_{1}\right):=\left(\Phi_{0}(a) x_{0}, \Phi_{1}(a) x_{1}\right) .
$$

Then $\Phi$ is $\gamma$-bounded if and only if both $\Phi_{0}$ and $\Phi_{1}$ are $\gamma$-bounded.

Proof. If $\Phi$ is $\gamma$-bounded, one can conclude the $\gamma$-boundedness of $\Phi_{0}$ and $\Phi_{1}$ be considering vectors $\left(x_{1,0}, 0\right), \ldots,\left(x_{n, 0}, 0\right) \in X$ and $\left(0, x_{1,1}\right), \ldots,\left(0, x_{n, 1}\right) \in X$ and applying 
Definition 2.6.8 to $\Phi$. For the reverse implication let $n \in \mathbb{N}, x_{1}, \ldots, x_{n} \in X_{0} \oplus X_{1}$ with $x_{k}=\left(x_{k, 0}, x_{k, 1}\right)$, let $a_{1} \ldots, a_{n} \in \mathcal{A}$ with $\left\|a_{k}\right\|_{\mathcal{A}} \leq 1$, and let $\gamma_{1}, \ldots, \gamma_{n}$ be independent Gaussians. Then, with $X:=X_{0} \oplus X_{1}$,

$$
\begin{gathered}
\left(\mathbb{E}\left\|\sum_{k=1}^{n} \gamma_{k} \Phi\left(a_{k}\right) x_{k}\right\|_{X}^{2}\right)^{\frac{1}{2}} \bar{\sim}\left(\mathbb{E}\left\|\sum_{k=1}^{n} \gamma_{k} \Phi_{0}\left(a_{k}\right) x_{k, 0}\right\|_{X_{0}}^{2}\right)^{\frac{1}{2}}+\left(\mathbb{E}\left\|\sum_{k=1}^{n} \gamma_{k} \Phi_{1}\left(a_{k}\right) x_{k, 1}\right\|_{X_{1}}^{2}\right)^{\frac{1}{2}} \\
\lesssim \Phi_{0}, \Phi_{1}\left(\mathbb{E}\left\|\sum_{k=1}^{n} \gamma_{k} x_{k, 0}\right\|_{X_{0}}^{2}\right)^{\frac{1}{2}}+\left(\mathbb{E}\left\|\sum_{k=1}^{n} \gamma_{k} x_{k, 1}\right\|_{X_{1}}^{2}\right)^{\frac{1}{2}} \approx\left(\mathbb{E}\left\|\sum_{k=1}^{n} \gamma_{k} x_{k}\right\|_{X}^{2}\right)^{\frac{1}{2}} \cdot \square
\end{gathered}
$$




\section{Chapter 3}

\section{Generalized Hörmander and Sobolev Functions}

For a bounded measurable function $g: \mathbb{R}_{+} \rightarrow \mathbb{C}$ the classical Hörmander condition of order $N \in \mathbb{N}$ reads

$$
\sup _{R>0} \int_{\frac{R}{2}}^{2 R}\left|s^{k} g^{(k)}(s)\right|^{2} \frac{\mathrm{d} s}{s}<\infty \quad(k=0, \ldots, N),
$$

see [28, (2.2.4)]. One can show (see for example [32, Proposition 4.11]) that this is equivalent to the condition

$$
\sup _{t>0}\|\eta \cdot g(t \mathbf{s})\|_{\mathrm{W}^{N, 2}(\mathbb{R})}<\infty
$$

where $0 \neq \eta \in \mathrm{C}_{\mathrm{c}}^{\infty}\left(\mathbb{R}_{+}\right)$, and where $\mathrm{W}^{N, 2}(\mathbb{R})$ is the classical $\mathrm{L}^{2}$-Sobolev space. This condition does not depend on the particular choice of $\eta$. That is, if $(3.0 .2)$ is satisfied for one non-zero test function with support in $\mathbb{R}_{+}$, then 3.0 .2 holds for every other test function with support in $\mathbb{R}_{+}$as well. In order to pass from integer orders $N \in \mathbb{N}$ to non-integer orders $\alpha>\frac{1}{2}$, one replaces the classical Sobolev spaces with fractional Sobolev spaces, i.e., one requires that

$$
\sup _{t>0}\|\eta \cdot g(t \mathbf{s})\|_{\mathrm{W}^{\alpha, 2}(\mathbb{R})}<\infty .
$$

This last formulation is well known and has been used by many authors, see for example [38, (1.1)], [25], 77, (1)], [43], and [5]. See also [54] and [36], where Sobolev spaces $\mathrm{W}^{\alpha, p}(\mathbb{R})$ for a wider range of $p$ are considered.

In the literature, one can also find a Hörmander condition for holomorphic functions defined on a sector (see [17], [39], 47], and [6]). Namely, for $\omega \in(0, \pi)$ and a function $g \in \mathrm{H}^{\infty}\left(\mathrm{S}_{\omega}\right) \cap \mathrm{C}_{\mathrm{b}}\left(\overline{\mathrm{S}}_{\omega} \backslash\{0\}\right)$ the Hörmander condition of order $\alpha>\frac{1}{2}$ reads

$$
\sup _{t>0}\left\|\eta \cdot g\left(t \mathrm{e}^{ \pm \mathrm{i} \omega} \mathbf{s}\right)\right\|_{\mathrm{W}^{\alpha, 2}(\mathbb{R})}<\infty .
$$


In other words, one requires the restrictions of $g$ to the upper and lower boundary of $\mathrm{S}_{\omega}$ to be classical Hörmander functions in the sense of (3.0.3).

In this chapter we aim for a theory of holomorphic Hörmander conditions to lay the groundwork for the functional calculus presented in Chapter 4 . We deviate from the classical setting and concentrate on functions on $\mathbb{R}$ and $\mathrm{St}_{\omega}$ (for $\omega>0$ ) instead of functions on $\mathbb{R}_{+}$or $\mathrm{S}_{\omega}$ (for $\omega \in(0, \pi)$ ). At the same time we establish a natural correspondence between the real line and the positive real line, and the strip and the sectorial case. (For the non-holomorphic case this idea can already be found in [32, 34] and [35] for example.)

To motivate this change of perspective, let us take again condition (3.0.1) and make a change of variables by considering $f:=g \circ \mathrm{e}^{\mathbf{s}}$ instead. Then (3.0.1) rewrites to

$$
\sup _{t \in \mathbb{R}} \int_{t-1}^{t+1}\left|f^{(k)}(s)\right|^{2} \mathrm{~d} s<\infty \quad(k=0, \ldots, N) .
$$

Conversely, if a function $f: \mathbb{R} \rightarrow \mathbb{C}$ satisfies (3.0.5), then one can easily show that $g:=$ $f \circ \log$ satisfies (3.0.1). This already shows that there is a natural exp-log-correspondence between the conditions 3.0.1 and 3.0.5), where the multiplicative structure of $\mathbb{R}_{+}$is exchanged for the additive structure of $\mathbb{R}$.

The natural generalization of 3.0 .5 from an integer order $N \in \mathbb{N}$ to a non-integer order $\alpha>\frac{1}{2}$ is given by

$$
\sup _{t \in \mathbb{R}}\left\|\eta \cdot \tau_{t} f\right\|_{\mathrm{W}^{\alpha, 2}(\mathbb{R})}=\sup _{t \in \mathbb{R}}\left\|\tau_{t} \eta \cdot f\right\|_{\mathrm{W}^{\alpha, 2}(\mathbb{R})}<\infty,
$$

where $0 \neq \eta \in \mathrm{C}_{\mathrm{c}}^{\infty}(\mathbb{R})$ is again arbitrary. The exp-log-correspondence between (3.0.1) and (3.0.5) extends to the more general conditions $(3.0 .3)$ and (3.0.6). That is, a function $f: \mathbb{R}_{+} \rightarrow \mathbb{C}$ satisfies (3.0.3) if and only if $f \circ \mathrm{e}^{\mathbf{s}}$ satisfies (3.0.6). This follows from the fact that for any function $f: \mathbb{R}_{+} \rightarrow \mathbb{C}$ one has

$$
\sup _{t>0}\|\eta \cdot f(t \mathbf{s})\|_{\mathrm{W}^{\alpha, 2}(\mathbb{R})} \approx \sup _{t>0}\left\|\tilde{\eta} \cdot f\left(t \mathrm{e}^{\mathbf{s}}\right)\right\|_{\mathrm{W}^{\alpha, 2}(\mathbb{R})},
$$

where $0 \neq \eta \in \mathrm{C}_{\mathrm{c}}^{\infty}\left(\mathbb{R}_{+}\right)$and $0 \neq \tilde{\eta} \in \mathrm{C}_{\mathrm{c}}^{\infty}(\mathbb{R})($ see $[32$, (4.13)]).

Condition (3.0.4) suggests that a Hörmander function on the strip $\mathrm{St}_{\omega}$ should be a function $f \in \mathrm{H}^{\infty}\left(\overrightarrow{\mathrm{St}}_{\omega}\right)$ satisfying

$$
\sup _{t \in \mathbb{R}}\left\|\tau_{t} \eta \cdot g(\mathbf{s} \pm \mathrm{i} \omega)\right\|_{\mathrm{W}^{\alpha, 2}(\mathbb{R})}<\infty .
$$

However, we will introduce generalized Hörmander spaces on strips in a different way that puts more emphasis on the connection to Sobolev spaces. Looking again at condition (3.0.6), Hörmander functions are a special class of "local" Sobolev functions. Given a function $0 \neq \eta \in \mathrm{C}_{\mathrm{c}}^{\infty}(\mathbb{R})$, a function $f: \mathbb{R} \rightarrow \mathbb{C}$ satisfies 3.0.6) if and only if $\tau_{t} \eta \cdot f \in$ $\mathrm{W}^{\alpha, 2}(\mathbb{R})$ for all $t \in \mathbb{R}$ and the family $\left(\tau_{t} \eta \cdot f\right)_{t \in \mathbb{R}}$ is bounded in $\mathrm{W}^{\alpha, 2}(\mathbb{R})$.

Motivated by this, we first develop a notion of (generalized) Sobolev functions on a strip. Then, generalized Hörmander functions on a strip are introduced as the class 
of "local" Sobolev functions whose local Sobolev norms are uniformly bounded. Here, the deciding factor is that we delegate the role of test functions as the "universal" algebraic regularizers to the holomorphic class of $\mathrm{H}_{0}^{\infty}$-functions instead. This leads to a Hörmander condition which can be equivalenty described in the spirit of (3.0.7) (see Proposition 3.3.16) so that the intuition behind (3.0.7) is still the right one.

The shift from test functions to $\mathrm{H}_{0}^{\infty}$-functions is crucial for the construction of the Hörmander functional calculus in the next chapter as it essentially allows us to pass from 0 -strip type and 0-sectorial operators to operators of strip type or angle of sectoriality greater than zero.

Chapter 3 is divided into four sections. In Section 3.1, so-called admissible weights are introduced and investigated. Based on these objects, generalized Sobolev and Hörmander spaces on strips are defined and discussed in Sections 3.2 and 3.3 . This chapter ends with Section 3.4, where we come back to Hörmander functions on sectors and discuss how our framework covers the classical sectorial condition (3.0.4).

\subsection{Admissible Weights}

Classical Sobolev spaces can be described via the (inverse) Fourier transform:

$$
\mathrm{W}^{N, 2}(\mathbb{R})=\left\{f \in \mathrm{L}^{2}(\mathbb{R}) \mid(1+|\mathbf{s}|)^{N} f^{\vee} \in \mathrm{L}^{2}(\mathbb{R})\right\} \quad(N \in \mathbb{N}) .
$$

Fractional Sobolev spaces are obtained by considering real powers instead of integer powers in (3.1.1), that is,

$$
\mathrm{W}^{\alpha, 2}(\mathbb{R})=\left\{f \in \mathrm{L}^{2}(\mathbb{R}) \mid(1+|\mathbf{s}|)^{\alpha} f^{\vee} \in \mathrm{L}^{2}(\mathbb{R})\right\} \quad\left(\alpha>\frac{1}{2}\right),
$$

see for example [20, p. 13 - 14]. Taking this idea one step further, we define a class of Sobolev spaces in Section 3.2 where the polynomial weights $(1+|\mathbf{s}|)^{\alpha}$ are replaced by a more general class of functions called admissible weights. (This idea can already be found in a short note of Strichartz, see [51].)

Definition 3.1.1. A measurable function $v: \mathbb{R} \rightarrow(0, \infty)$ is called admissible if $v$ has the following properties:

1) $\forall s \in \mathbb{R}: \quad v(s) \geq 1$;

2) $\exists c>0 \forall s, t \in \mathbb{R}: \quad v(s+t) \leq c(v(s)+v(t))$.

We write

$$
M_{v}:=\sup _{s, t \in \mathbb{R}} \frac{v(s+t)}{v(s)+v(t)}
$$

for the smallest admissible constant in 2). An admissible function $v: \mathbb{R} \rightarrow[1, \infty)$ is called an admissible weight if additionally

3) $\frac{1}{v} \in \mathrm{L}^{2}(\mathbb{R})$. 


\section{Remarks 3.1.2.}

1) In the literature, fractional Sobolev spaces $W^{\alpha, 2}(\mathbb{R})$ are also considered for real orders $\alpha \in \mathbb{R}$. We only restrict ourselves to the case $\alpha>\frac{1}{2}$, since $\mathrm{W}^{\alpha, 2}(\mathbb{R})$ is an algebra with respect to pointwise multiplication if and only if $\alpha>\frac{1}{2}$ (see [50, Theorem 2.1] and also Proposition 3.2.18). This is reflected in property 3) of Definition 3.1.1. As we base a functional calculus on Sobolev spaces later on, only the case $\alpha>\frac{1}{2}$ is of interest.

2) The class of admissible weights was already considered by Strichartz in [51].

Before we give examples for admissible weights, note the following characterization of property 2) of Definition 3.1.1 for functions which satisfy a certain monotonicity condition.

Lemma 3.1.3. Let $v: \mathbb{R} \rightarrow(0, \infty)$ be a function which is decreasing on $(-\infty, 0]$ and increasing on $[0, \infty)$. Then the following statements are equivalent:

(i) $\exists c \geq 0 \forall s \in \mathbb{R}: \quad v(2 s) \leq c v(s)$;

(ii) $\exists c \geq 0 \forall s, t \in \mathbb{R}: \quad v(s+t) \leq c \max \{v(s), v(t)\}$;

(iii) $\exists c \geq 0 \forall s, t \in \mathbb{R}: \quad v(s+t) \leq c(v(s)+v(t))$.

Proof. Without loss of generality we may suppose that $v(0)=1$. The implications (ii) $\Rightarrow$ (iii) and (iii) $\Rightarrow$ (i) are clear. Suppose that (i) holds and fix $s, t \in \mathbb{R}$ with $s \leq t$. Then $2 s \leq s+t \leq 2 t$. Hence, if $s+t \geq 0$, then

$$
v(s+t) \leq v(2 t) \leq c v(t)
$$

by the monotonicity of $v$ on $[0, \infty)$ and (i). If $s+t<0$, then

$$
v(s+t) \leq v(2 s) \leq c v(s)
$$

Statement (ii) follows readily.

The next lemma is immediate which is why we omit the proof.

Lemma 3.1.4. Let $v, \tilde{v}: \mathbb{R} \rightarrow[1, \infty)$ be admissible functions. Then the following statements hold:

a) For every $\alpha \geq 0$ the function $v^{\alpha}$ is admissible.

b) If both $v$ and $\tilde{v}$ are decreasing on $(-\infty, 0]$ and increasing on $[0, \infty)$, then $v \widetilde{v}$ is an admissible function.

c) For all $\theta, \tilde{\theta} \geq 0$ with $\theta+\tilde{\theta} \geq 1$ the function $\theta v+\tilde{\theta} \tilde{v}$ is admissible.

\section{Examples 3.1.5.}

1) (Polynomial weights) Let $v:=1+|\mathbf{s}|$. Then $v$ is decreasing on $(-\infty, 0]$ and increasing on $[0, \infty)$. As

$$
v(2 s)=1+|2 s| \leq 2(1+|s|)
$$


for every $s \in \mathbb{R}$, Lemma 3.1 .3 yields that $v$ is an admissible function. Consequently, by Lemma 3.1.4 a), $v_{\alpha}:=v^{\alpha}$ is an admissible function for each $\alpha \geq 0$. In particular, $v_{\alpha}$ is an admissible weight for every $\alpha>\frac{1}{2}$.

2) (Mixed weights) Let $v_{\log }:=\ln (\mathrm{e}+|\mathbf{s}|)$. Then $v_{\log }$ is decreasing on $(-\infty, 0]$ and increasing on $[0, \infty)$. Observe that, for each $s \in \mathbb{R}$,

$$
\frac{v_{\log }(2 s)}{v_{\log (s)}(s)}=\frac{\ln \left(\frac{\mathrm{e}}{2}+|s|\right)+\ln 2}{\ln (\mathrm{e}+|s|)} \leq 1+\frac{\ln 2}{\ln (\mathrm{e}+|s|)} \leq 1+\ln 2 .
$$

Hence, by Lemma 3.1.3, $v_{\log }$ has property 2) of Definition 3.1.1 and is therefore an admissible function. By Lemma 3.1.4.

$$
v_{\alpha, \beta}:=(1+|\mathbf{s}|)^{\alpha} v_{\log }^{\beta}=(1+|\mathbf{s}|)^{\alpha}(\ln (\mathrm{e}+|\mathbf{s}|))^{\beta}
$$

is an admissible function for each $\alpha, \beta>0$. In particular, whenever either $\alpha>\frac{1}{2}$ and $\beta \geq 0$, or $\alpha=\frac{1}{2}$ and $\beta>1$, the function $v_{\alpha, \beta}$ is an admissible weight.

3) We give an example for a function which is not admissible. The function

$$
v:=\exp (\sqrt{|\mathbf{s}|})
$$

satisfies 1) and 3) of Definition 3.1.1. Moreover, $v$ is decreasing on $(-\infty, 0]$ and increasing on $[0, \infty)$. However,

$$
\frac{v(2 s)}{v(s)}=\exp ((\sqrt{2}-1) \sqrt{|s|}) \stackrel{s \rightarrow \pm \infty}{\longrightarrow} \infty .
$$

Hence, $v$ misses 2) and therefore cannot be an admissible function.

The next two lemmata show that admissible functions grow at most polynomially fast. Hence, it is no surprise that the function from Example 3.1.5.3) is not admissible.

Lemma 3.1.6. Let $v: \mathbb{R} \rightarrow[0, \infty)$ be a measurable function with property 2$)$ of Definition 3.1.1. Then $v$ is locally bounded on $\mathbb{R}$.

Proof. It suffices to show that $v$ is locally bounded at zero, i.e., that there is $\varepsilon>0$ such that $v$ is bounded on $(-\varepsilon, \varepsilon)$. Choose $R>0$ such that

$$
\Omega_{R}:=\{s \in \mathbb{R} \mid v(s)+v(-s) \leq R\}
$$

has Lebesgue measure greater than zero. Then, by Steinhaus's theorem (see [52]), there is $\varepsilon>0$ with

$$
(-\varepsilon, \varepsilon) \subseteq\left\{s-t \mid s, t \in \Omega_{R}\right\}=\Omega_{R}-\Omega_{R} .
$$

By property 2), $v$ is bounded on $\Omega_{R}-\Omega_{R}$, whence $v$ is locally bounded in zero. 
Lemma 3.1.7. Every admissible function grows at most polynomially. More precisely: Let $v: \mathbb{R} \rightarrow(0, \infty)$ be a function which is locally bounded at zero, and suppose that there is a constant $c>0$ with

$$
v(2 s) \leq c v(s) \quad(s \in \mathbb{R})
$$

Then there is $\alpha \geq 0$ with

$$
\sup _{s \in \mathbb{R}} \frac{v(s)}{(1+|s|)^{\alpha}}<\infty .
$$

Proof. Without loss of generality, suppose that $v$ is symmetric. Otherwise, consider the function $\widetilde{v}:=\max \{v(\mathbf{s}), v(-\mathbf{s})\}$ instead. We may further suppose that $v$ is bounded on the interval $[-2,2]$. Choose $\alpha>0$ big enough so that $c \leq 2^{\alpha}$. For $0 \leq r \leq 1$ and $n \in \mathbb{N}_{0}$ observe that

$$
\frac{v\left(2^{n} 2^{r}\right)}{\left(2^{n} 2^{r}\right)^{\alpha}} \leq c^{n} \frac{v\left(2^{r}\right)}{2^{\alpha n} 2^{\alpha r}}=\left(\frac{c}{2^{\alpha}}\right)^{n} \frac{v\left(2^{r}\right)}{2^{\alpha r}} \leq \sup _{1 \leq s \leq 2} v(s) .
$$

As each $s \geq 1$ has a representation $s=2^{n+r}$ for some $n \in \mathbb{N}_{0}$ and $r \in[0,1]$, we obtain

$$
\sup _{s \geq 1} \frac{v(s)}{s^{\alpha}} \leq \sup _{1 \leq s \leq 2} v(s) .
$$

Consequently,

$$
\sup _{s \in \mathbb{R}} \frac{v(s)}{(1+|s|)^{\alpha}}=\sup _{s \geq 0} \frac{v(s)}{(1+s)^{\alpha}} \leq \sup _{0 \leq s \leq 2} v(s)<\infty,
$$

which yields the claim.

We conclude this section with two more observations on admissible functions. First, every admissible function is submultiplicative (at the cost of a constant).

Lemma 3.1.8. Let $v: \mathbb{R} \rightarrow[1, \infty)$ be an admissible function. Then there is a constant $c^{\prime}>0$ such that

$$
v(s+t) \leq c^{\prime} v(s) v(t)
$$

for all $s, t \in \mathbb{R}$.

Proof. Let $s, t \in \mathbb{R}$. Then

$$
v(s+t) \leq M_{v}(v(s)+v(t)) \leq M_{v}(v(s) v(t)+v(s) v(t))=2 M_{v} v(s) v(t),
$$

where we have used that $v(s), v(t) \geq 1$. Hence, the claim follows with $c^{\prime}=2 M_{v}$.

Every admissible function is similar to a smooth admissible function in the following sense:

Lemma 3.1.9. Let $v: \mathbb{R} \rightarrow[1, \infty)$ be an admissible function. Then there is a constant $c>0$ and an admissible function $\tilde{v} \in \mathrm{C}^{\infty}(\mathbb{R})$ with

$$
\frac{1}{c} \tilde{v} \leq v \leq c \tilde{v}
$$


Proof. Let $\eta \in \mathrm{C}_{\mathrm{c}}^{\infty}(\mathbb{R})$ be an even, positive function with

$$
\int_{\mathbb{R}} \eta(r) \mathrm{d} r=1,
$$

and such that $\left.\eta\right|_{[0, \infty)}$ is decreasing. Note that by this monotonicity property one has

$$
\eta(2 t) \leq \eta(t) \quad(t \in \mathbb{R})
$$

Set $\tilde{v}:=v * \eta$. Then $1 \leq \tilde{v} \in \mathrm{C}^{\infty}(\mathbb{R})$, and one has

$$
\begin{aligned}
\tilde{v}(s+t) & =\int_{\mathbb{R}} v(s+t-r) \eta(r) \mathrm{d} r \\
& \leq M_{v}\left(\int_{\mathbb{R}} v\left(s-\frac{r}{2}\right) \eta(r) \mathrm{d} r+\int_{\mathbb{R}} v\left(t-\frac{r}{2}\right) \eta(r) \mathrm{d} r\right) \\
& =2 M_{v}\left(\int_{\mathbb{R}} v(s-r) \eta(2 r) \mathrm{d} r+\int_{\mathbb{R}} v(t-r) \eta(2 r) \mathrm{d} r\right) \\
& \leq 2 M_{v}(\tilde{v}(s)+\tilde{v}(t))
\end{aligned}
$$

for all $s, t \in \mathbb{R}$. Hence, $\tilde{v}$ is an admissible function. Now, choose $c^{\prime}>0$ for $v$ as in Lemma 3.1 .8 and set $K:=\operatorname{supp}(\eta)$. Then, for all $s \in \mathbb{R}$,

$$
v(s)=\int_{\mathbb{R}} v(s) \eta(r) \mathrm{d} r \leq c^{\prime} \int_{\mathbb{R}} v(r) v(s-r) \eta(r) \mathrm{d} r \leq c^{\prime}\|v\|_{\infty, K} \tilde{v}(s),
$$

and

$$
\begin{aligned}
\tilde{v}(s) & =\int_{\mathbb{R}} v(s-r) \eta(r) \mathrm{d} r=\int_{\mathbb{R}} v(s+r) \eta(r) \mathrm{d} r \\
& \leq\left(c^{\prime} \int_{\mathbb{R}} v(r) \eta(r) \mathrm{d} r\right) v(s) \leq c^{\prime}\|v\|_{\infty, K} v(s) .
\end{aligned}
$$

This proves the claim.

Remark 3.1.10. As a consequence of Lemma 3.1.9, an admissible weight $v: \mathbb{R} \rightarrow[1, \infty)$, if not further specified, can always be assumed to be smooth.

\subsection{Generalized Sobolev Functions on a Strip}

As already mentioned in the beginning of Section 3.1, we now take classical fractional Sobolev spaces

$$
\mathrm{W}^{\alpha, 2}(\mathbb{R})=\left\{f \in \mathrm{L}^{2}(\mathbb{R}) \mid(1+|\mathbf{s}|)^{\alpha} f^{\vee} \in \mathrm{L}^{2}(\mathbb{R})\right\} \quad\left(\alpha>\frac{1}{2}\right)
$$

and replace the polynomial weights $(1+|\mathbf{s}|)^{\alpha}$ with admissible weights $v: \mathbb{R} \rightarrow[1, \infty)$ together with an additional exponential factor $\mathrm{e}^{\omega \mid \mathbf{s}}$. 
Definition 3.2.1. Let $\omega \geq 0$, and let $v: \mathbb{R} \rightarrow[1, \infty)$ be an admissible weight. We define the space

$$
\mathrm{W}_{v, \omega}^{2}(\mathbb{R}):=\left\{f \in \mathrm{L}^{2}(\mathbb{R})\left|v \mathrm{e}^{\omega \mid \mathbf{s}}\right| f^{\vee} \in \mathrm{L}^{2}(\mathbb{R})\right\}
$$

and equip it with the norm

$$
\|f\|_{v, \omega}:=\left\|v \mathrm{e}^{\omega|\mathbf{s}|} f^{\vee}\right\|_{2}=\left(\int_{\mathbb{R}}\left|v(s) \mathrm{e}^{\omega|s|} f^{\vee}(s)\right|^{2} \mathrm{~d} s\right)^{\frac{1}{2}} .
$$

\section{Remarks 3.2.2.}

1) We make only temporary use of the notation $\mathrm{W}_{v, \omega}^{2}(\mathbb{R})$ and $\|\cdot\|_{v, \omega}$ as we shall show in Corollary 3.2 .13 that each function $f \in \mathrm{W}_{v, \omega}^{2}(\mathbb{R})$ extends uniquely to a continuous function on $\overline{\mathrm{St}}_{\omega}$ that is holomorphic on $\mathrm{St}_{\omega}$ (if $\omega>0$ ). From that point on, we shall use $\mathrm{W}_{v}^{2}\left(\overline{\mathrm{St}}_{\omega}\right)$ and $\|\cdot\|_{\mathrm{W}_{v}^{2}(\overline{\mathrm{St}} \omega)}$ rather than $\mathrm{W}_{v, \omega}^{2}(\mathbb{R})$ and $\|\cdot\|_{v, \omega}$ to denote this space and norm (see Definition 3.2.14).

2) Clearly, $\mathrm{W}_{v, \omega}^{2}(\mathbb{R})$ is a Hilbert space and

$$
\mathcal{K}_{\omega, v}: \mathrm{W}_{v, \omega}^{2}(\mathbb{R}) \rightarrow \mathrm{L}^{2}(\mathbb{R}), \quad f \mapsto v \mathrm{e}^{\omega|\mathbf{s}|} f^{\vee}
$$

is an isometric isomorphism with inverse $\mathcal{K}_{\omega, v}^{-1} f=\mathcal{F}\left(\frac{\mathrm{e}^{-\omega|\mathbf{s}|}}{v} f\right)$.

3) By the remark above, the space $\mathrm{W}_{v, \omega}^{2}(\mathbb{R})$ is separable.

4) Different admissible weights lead to different spaces in the following sense: Let $\omega \geq$ 0 , and let $v_{0}, v_{1}: \mathbb{R} \rightarrow[1, \infty)$ be two admissible weights which are not comparable, i.e., one of the two functions $\frac{v_{0}}{v_{1}}$ or $\frac{v_{1}}{v_{0}}$ is unbounded. Then $\mathrm{W}_{v_{0}, \omega}^{2}(\mathbb{R}) \neq \mathrm{W}_{v_{1}, \omega}^{2}(\mathbb{R})$.

Proof. Suppose that $\frac{v_{0}}{v_{1}}$ is not bounded on $\mathbb{R}$. Choose $g \in \mathrm{L}^{2}(\mathbb{R})$ with $\frac{v_{0}}{v_{1}} g \notin \mathrm{L}^{2}(\mathbb{R})$ and set

$$
f:=\mathcal{F}\left(\frac{\mathrm{e}^{-\omega|\mathbf{s}|}}{v_{1}} g\right)=\mathcal{K}_{\omega, v_{1}}^{-1}(g) .
$$

Then $f \in \mathrm{W}_{v_{1}, \omega}^{2}(\mathbb{R})$. However, as

$$
\|f\|_{v_{0}, \omega}=\left\|\frac{v_{0}}{v_{1}} g\right\|_{2}=\infty
$$

one has $f \notin \mathrm{W}_{v_{0}, \omega}^{2}(\mathbb{R})$.

The next lemma gathers immediate embedding properties of the spaces $\mathrm{W}_{v, \omega}^{2}(\mathbb{R})$. The proof is straightforward and therefore omitted.

Lemma 3.2.3. Let $\theta>\omega \geq 0$, and let $v, \widetilde{v}: \mathbb{R} \rightarrow[1, \infty)$ be admissible weights with $v \lesssim \widetilde{v}$. Then the following statements hold:

a) The canonical embedding $\mathrm{W}_{v, \theta}^{2}(\mathbb{R}) \hookrightarrow \mathrm{W}_{v, \omega}^{2}(\mathbb{R})$ is contractive.

b) The canonical embedding $\mathrm{W}_{\widetilde{v}, \omega}^{2}(\mathbb{R}) \hookrightarrow \mathrm{W}_{v, \omega}^{2}(\mathbb{R})$ is continuous. 
Corollary 3.2.4. Let $v: \mathbb{R} \rightarrow[1, \infty)$ be an admissible weight. Then one has $\mathrm{C}_{\mathrm{c}}^{\infty}(\mathbb{R}) \subseteq$ $\mathrm{W}_{v, 0}^{2}(\mathbb{R})$.

Proof. By Lemma 3.1.7 there is $N \in \mathbb{N}$ with $v \lesssim(1+|\mathbf{s}|)^{N}$. Then

$$
\mathrm{C}_{\mathrm{c}}^{\infty}(\mathbb{R}) \subseteq \mathrm{W}^{N, 2}(\mathbb{R}) \subseteq \mathrm{W}_{v, 0}^{2}(\mathbb{R})
$$

Remark 3.2.5. Let $\omega>0$. Then one has $\mathrm{C}_{\mathrm{c}}^{\infty}(\mathbb{R}) \nsubseteq \mathrm{W}_{v, \omega}^{2}(\mathbb{R})$. Indeed, if $\omega>0$, then $\mathrm{W}_{v, \omega}^{2}(\mathbb{R})$-functions can be extended to holomorphic functions on $\mathrm{St}_{\omega}$ (see Corollary 3.2 .13 . Hence, $\mathrm{W}_{v, \omega}^{2}(\mathbb{R}) \cap \mathrm{C}_{\mathrm{c}}^{\infty}(\mathbb{R})=\{0\}$.

\section{$\mathrm{L}^{2}$-Boundary Conditions for Holomorphic Functions on a Strip}

Recall that for a function $f: \overline{\mathrm{St}}_{\omega} \rightarrow \mathbb{C}$ and $r \in[-\omega, \omega]$ we write

$$
f_{r}: \mathbb{R} \rightarrow \mathbb{C}, \quad f_{r}(s):=f(s+\mathrm{i} r),
$$

i.e., $f_{r}$ is essentially the restriction of $f$ to the line $\mathbb{R}+\mathrm{i} r$. In effect, $f_{0}=\left.f\right|_{\mathbb{R}}$. In this subsection, we show that under certain assumptions on $f$ one has

$$
\mathrm{e}^{r \mathbf{s}} f^{\vee}=\left(f_{r}\right)^{\vee}
$$

in a suitable sense, where $f^{\vee}=\left(f_{0}\right)^{\vee}$. See Lemma 3.2 .8 for the exact statement. In other words, scaling the inverse Fourier transform of $f$ by the exponential factor $\mathrm{e}^{r \mathbf{s}}$ yields the inverse Fourier transform of the restriction $f_{r}$. Thus, estimates for $f^{\vee}$ can be obtained from estimates for $\left(f_{r}\right)^{\vee}$. This idea can be found in the proof of [17, Theorem 2.2] for example. There, the authors consider the Mellin transform instead of the Fourier transform.

Recall the notation

$$
\psi=\mathrm{e}^{-\mathbf{z}^{2}} .
$$

We note the following lemma for future reference.

Lemma 3.2.6. Let $r \in \mathbb{R}$, and set $\psi_{n, r}:=\left(\psi\left(\frac{\mathrm{z}}{n}\right)\right)_{r}=\psi\left(\frac{\mathbf{s}}{n}+\mathrm{i} \frac{r}{n}\right)$. Then

$$
f=\lim _{n \rightarrow \infty} \psi_{n, r} f
$$

in $\mathrm{L}^{2}(\mathbb{R})$ for each $f \in \mathrm{L}^{2}(\mathbb{R})$.

Proof. This follows directly from the dominated convergence theorem.

For functions in $\mathrm{H}_{0}^{\infty}\left(\mathrm{St}_{\omega}\right) \cap \mathrm{C}_{\mathrm{b}}\left(\overline{\mathrm{St}}_{\omega}\right)$ one has the following identity:

Lemma 3.2.7. Let $\omega>0$, and let $\psi \in \mathrm{H}_{0}^{\infty}\left(\mathrm{St}_{\omega}\right) \cap \mathrm{C}_{\mathrm{b}}\left(\overline{\mathrm{St}}_{\omega}\right)$. Then

$$
\left(\psi_{r}\right)^{\vee}=\mathrm{e}^{r \mathbf{s}} \psi^{\vee}
$$

for each $r \in[-\omega, \omega]$. 
Proof. By Cauchy's integral theorem,

$$
\psi^{\vee}(s)=\frac{1}{2 \pi} \int_{\mathbb{R}} \psi(t) \mathrm{e}^{\mathrm{i} s t} \mathrm{~d} t=\frac{1}{2 \pi} \int_{\mathbb{R}+\mathrm{i} r} \psi(z) \mathrm{e}^{\mathrm{i} s z} \mathrm{~d} z=\mathrm{e}^{-r s}\left(\psi_{r}\right)^{\vee}(s)
$$

for all $s \in \mathbb{R}$.

The next lemma extends the previous one to $\mathrm{H}^{\infty}\left(\overline{\mathrm{St}}_{\omega}\right)$-functions which are square integrable on the boundary of $\overline{\mathrm{St}}_{\omega}$.

Lemma 3.2.8 (Scaling lemma). Let $\omega>0$, and let $f \in \mathrm{H}^{\infty}\left(\overline{\mathrm{St}}_{\omega}\right)$. Then the following statements are equivalent:

(i) $f_{r} \in \mathrm{L}^{2}(\mathbb{R})$ for all $r \in[-\omega, \omega]$;

(ii) $f_{\omega}, f_{-\omega} \in \mathrm{L}^{2}(\mathbb{R})$;

(iii) $f, \mathrm{e}^{\omega \mid \mathbf{s}} f^{\vee} \in \mathrm{L}^{2}(\mathbb{R})$.

In this case,

$$
\mathrm{e}^{r \mathbf{s}} f^{\vee}=\left(f_{r}\right)^{\vee}
$$

and

$$
\mathrm{e}^{r|\mathbf{s}|} f^{\vee}=\left(f_{-r}\right)^{\vee} \mathbb{1}_{(-\infty, 0)}+\left(f_{r}\right)^{\vee} \mathbb{1}_{(0, \infty)}
$$

in $\mathrm{L}^{2}(\mathbb{R})$ for each $r \in[-\omega, \omega]$.

Proof. The implication from (i) to (ii) is clear. Suppose that (ii) holds. We prove (iii). By the Cauchy-Gauss representation formula (see [21, Section 8]) we have

$$
\begin{aligned}
\int_{\mathbb{R}}|f(t)|^{2} \mathrm{~d} t & =\int_{\mathbb{R}}\left|\frac{1}{2 \pi \mathrm{i}} \int_{\partial \mathrm{St}_{\omega}} \frac{f(z) \mathrm{e}^{-(z-t)^{2}}}{z-t} \mathrm{~d} z\right|^{2} \mathrm{~d} t \\
& \leq c_{0} \int_{\mathbb{R}} \int_{\partial \mathrm{St}_{\omega}}\left|f(z) \mathrm{e}^{-(z-t)^{2}}\right|^{2}|\mathrm{~d} z| \mathrm{d} t \\
& =c_{0} \int_{\partial \operatorname{St}_{\omega}}|f(z)|^{2} \int_{\mathbb{R}}\left|\mathrm{e}^{-(z-t)^{2}}\right|^{2} \mathrm{~d} t|\mathrm{~d} z|,
\end{aligned}
$$

where

$$
c_{0}=\frac{1}{4 \pi^{2}} \int_{\partial \mathrm{St}_{\omega}}\left|\frac{1}{z-t}\right|^{2}|\mathrm{~d} z|=\frac{1}{2 \pi^{2}} \int_{\mathbb{R}} \frac{1}{\omega^{2}+s^{2}} \mathrm{~d} s=\frac{1}{2 \pi \omega} .
$$

Note that

$$
\int_{\mathbb{R}}\left|\mathrm{e}^{-(z-t)^{2}}\right|^{2} \mathrm{~d} t=\mathrm{e}^{2 \omega^{2}} \int_{\mathbb{R}} \mathrm{e}^{-2 s^{2}} \mathrm{~d} s=\frac{\mathrm{e}^{2 \omega^{2}} \sqrt{\pi}}{\sqrt{2}}=: c_{1}
$$

for each $z \in \partial \mathrm{St}_{\omega}$, so that

$$
\|f\|_{2}^{2} \leq c_{0} c_{1} \int_{\partial \mathrm{St}_{\omega}}|f(z)|^{2}|\mathrm{~d} z|=c_{0} c_{1}\left(\left\|f_{\omega}\right\|_{2}^{2}+\left\|f_{-\omega}\right\|_{2}^{2}\right)<\infty,
$$


i.e. $f \in \mathrm{L}^{2}(\mathbb{R})$. Next, fix $r \in\{-\omega, \omega\}$ and let $\psi_{n}$ and $\psi_{n, r}$ as in Lemma 3.2.6. Note that $\psi_{n} f \in \mathrm{H}_{0}^{\infty}\left(\mathrm{St}_{\omega}\right) \cap \mathrm{C}_{\mathrm{b}}\left(\overline{\mathrm{St}}_{\omega}\right)$ for each $n \in \mathbb{N}$. Hence, whenever $\eta \in \mathrm{C}_{\mathrm{c}}(\mathbb{R})$,

$$
\begin{aligned}
\int_{\mathbb{R}} \eta(s)\left(f_{r}\right)^{\vee}(s) \mathrm{d} s & =\lim _{n \rightarrow \infty} \int_{\mathbb{R}} \eta(s)\left(\psi_{n, r} f_{r}\right)^{\vee}(s) \mathrm{d} s \\
& =\lim _{n \rightarrow \infty} \int_{\mathbb{R}} \eta(s) \mathrm{e}^{r s}\left(\psi_{n} f\right)^{\vee}(s) \mathrm{d} s \\
& =\int_{\mathbb{R}} \eta(s) \mathrm{e}^{r s} f^{\vee}(s) \mathrm{d} s
\end{aligned}
$$

by Lemma 3.2.7. As $\mathrm{C}_{\mathrm{c}}(\mathbb{R})$ is dense in $\mathrm{L}^{2}(\mathbb{R})$, this yields that $\mathrm{e}^{r \mathbf{s}} f^{\vee} \in \mathrm{L}^{2}(\mathbb{R})$ and that $\left(f_{r}\right)^{\vee}=\mathrm{e}^{r \mathrm{~s}} f^{\vee}$ in $\mathrm{L}^{2}(\mathbb{R})$, i.e. 3.2 .3 .

For the implication from (iii) to (i) fix $r \in[-\omega, \omega]$. By (iii), $\mathrm{e}^{r \mathrm{~s}} f^{\vee} \in \mathrm{L}^{2}(\mathbb{R})$. Let $\psi_{n}$ and $\psi_{n, r}$ be as before and observe that for each $\eta \in \mathrm{C}_{\mathrm{c}}^{\infty}(\mathbb{R})$,

$$
\begin{aligned}
\int_{\mathbb{R}} \widehat{\eta}(s) \overline{f_{r}(s)} \mathrm{d} s & =\lim _{n \rightarrow \infty} \int_{\mathbb{R}} \widehat{\eta}(s) \overline{\psi_{n, r}(s) f_{r}(s)} \mathrm{d} s \\
& =\lim _{n \rightarrow \infty} 2 \pi \int_{\mathbb{R}} \eta(s) \overline{\left(\psi_{n, r} f_{r}\right)^{\vee}(s)} \mathrm{d} s \\
& =\lim _{n \rightarrow \infty} 2 \pi \int_{\mathbb{R}} \eta(s) \overline{\mathrm{e}^{r s}\left(\psi_{n} f\right)^{\vee}(s)} \mathrm{d} s \\
& =2 \pi \int_{\mathbb{R}} \eta(s) \overline{\mathrm{e}^{r s} f^{\vee}(s)} \mathrm{d} s \\
& =\int_{\mathbb{R}} \widehat{\eta}(s) \overline{\mathcal{F}\left(\mathrm{e}^{r \mathbf{s}} f^{\vee}\right)(s)} \mathrm{d} s .
\end{aligned}
$$

Hence,

$$
f_{r}=\mathcal{F}\left(\mathrm{e}^{r \mathbf{s}} f^{\vee}\right) \in \mathrm{L}^{2}(\mathbb{R}) .
$$

In particular, $\left(f_{r}\right)^{\vee}=\mathrm{e}^{r \mathbf{s}} f^{\vee}$. Identity (3.2.4) follows easily from identity (3.2.3).

Remark 3.2.9. By Fatou's theorem, each function in $\mathrm{H}^{\infty}\left(\mathrm{St}_{\omega}\right)$ admits an $\mathrm{L}^{\infty}$-trace on the upper and the lower boundary of $\mathrm{St}_{\omega}$, say $\widetilde{f}_{\omega}$ and $\widetilde{f}_{-\omega}$. It is possible to generalize Lemma 3.2.8 to $\mathrm{H}^{\infty}\left(\mathrm{St}_{\omega}\right)$-functions by replacing $f_{ \pm \omega}$ with $\widetilde{f}_{ \pm \omega}$ in the statements (i) and (ii) and identities (3.2.3) and (3.2.4). However, for our purposes Lemma 3.2.8 is not needed in this generality. In the next subsection it is shown that $\mathrm{W}_{v}^{2}\left(\overline{\mathrm{St}}_{\omega}\right)$-functions may always be extended to functions in $\mathrm{C}_{\mathrm{b}}\left(\overline{\mathrm{St}}_{\omega}\right)$. Hence, all functions of interest already admit a continuous extension to the boundary of $\mathrm{St}_{\omega}$.

The next lemma is a useful tool for the subsequent sections.

Lemma 3.2.10. Let $\theta>\omega \geq 0$, let $f \in \mathrm{H}^{\infty}\left(\overline{\mathrm{St}}_{\theta}\right)$ with $f_{\theta}, f_{-\theta} \in \mathrm{L}^{2}(\mathbb{R})$, and let $v: \mathbb{R} \rightarrow$ $[1, \infty)$ be an admissible weight. Then $f_{0} \in \mathrm{W}_{v, \omega}^{2}(\mathbb{R})$ with

$$
\left\|f_{0}\right\|_{v, \omega} \leq c_{\theta, \omega, v}\left(\left\|f_{-\theta}\right\|_{2}+\left\|f_{\theta}\right\|_{2}\right),
$$

where $c_{\theta, \omega, v}=\sup _{s \in \mathbb{R}}\left(\mathrm{e}^{(\omega-\theta)|s|} v(s)\right)$. 
Proof. First note that

$$
c:=c_{v, \omega, \theta}:=\sup _{s \in \mathbb{R}}\left(\mathrm{e}^{-(\theta-\omega)|s|} v(s)\right)<\infty
$$

as $\omega-\theta<0$ and $v$ grows at most polynomially by Lemma 3.1.7. Moreover,

$$
\mathrm{e}^{\theta|\mathbf{s}|} f^{\vee}=\left(f_{-\theta}\right)^{\vee} \mathbb{1}_{(-\infty, 0)}+\left(f_{\theta}\right)^{\vee} \mathbb{1}_{(0, \infty)}
$$

by identity (3.2.4). Hence,

$$
\begin{aligned}
\|f\|_{v, \omega}^{2} & =\int_{\mathbb{R}}\left|v(s) \mathrm{e}^{\omega|s|} f^{\vee}(s)\right|^{2} \mathrm{~d} s \leq c^{2} \int_{\mathbb{R}}\left|\mathrm{e}^{\theta|s|} f^{\vee}(s)\right|^{2} \mathrm{~d} s \\
& =c^{2}\left\|\left(f_{-\theta}\right)^{\vee} \mathbb{1}_{(-\infty, 0)}+\left(f_{\theta}\right)^{\vee} \mathbb{1}_{(0, \infty)}\right\|_{2}^{2} \leq c^{2}\left(\left\|f_{-\theta}\right\|_{2}+\left\|f_{\theta}\right\|_{2}\right)^{2} .
\end{aligned}
$$

In the last estimate we have also used Plancherel's theorem.

Corollary 3.2.11. Let $\omega \geq 0$, and let $v: \mathbb{R} \rightarrow[1, \infty)$ be an admissible weight. Then, for each $\psi \in \mathcal{E}\left[\mathrm{St}_{\omega}\right]$ one has $\psi_{0} \in \mathrm{W}_{v, \omega}^{2}(\mathbb{R})$.

Proof. Fix $\psi \in \mathcal{E}\left(\mathrm{St}_{\theta^{\prime}}\right)$ for some width $\theta^{\prime}>\omega$, and let $\theta \in\left(\omega, \theta^{\prime}\right)$. As one has

$$
f_{r} \in \mathrm{L}^{\infty}(\mathbb{R}) \cap \mathrm{L}^{1}(\mathbb{R}) \subseteq \mathrm{L}^{2}(\mathbb{R})
$$

for each $r \in\{-\theta, \theta\}$, the claim follows from Lemma 3.2.10.

\section{Holomorphic Extensions of Sobolev Functions}

Next we show that each $\mathrm{W}_{v, \omega}^{2}(\mathbb{R})$-function admits a unique extension to a function in $\mathrm{C}_{\mathrm{b}}\left(\overline{\mathrm{St}}_{\omega}\right)$ which, if $\omega>0$, is holomorphic on $\mathrm{St}_{\omega}$. Moreover, we prove for $\omega>0$ that the space $\mathrm{W}_{v, \omega}^{2}(\mathbb{R})$ can be identified with the space of all $\mathrm{H}^{\infty}\left(\overline{\mathrm{St}}_{\omega}\right)$-functions whose restrictions to the upper and lower boundary of the strip lie in $\mathrm{W}_{v, 0}^{2}(\mathbb{R})$ (see Proposition 3.2 .16 .

Lemma 3.2.12. Let $\omega \geq 0$, let $v: \mathbb{R} \rightarrow[1, \infty)$ be an admissible weight, and let $f \in$ $\mathrm{W}_{v, \omega}^{2}(\mathbb{R})$. Then $\mathrm{e}^{\omega|\mathbf{s}|} f^{\vee} \in \mathrm{L}^{1}(\mathbb{R})$ with

$$
\left\|\mathrm{e}^{\omega|\mathbf{s}|} f^{\vee}\right\|_{1} \leq\left\|\frac{1}{v}\right\|_{2}\|f\|_{v, \omega} .
$$

Proof. Note that

$$
\mathrm{e}^{\omega|\mathbf{s}|} f^{\vee}=\frac{1}{v}\left(v \mathrm{e}^{\omega \mid \mathbf{s}} f^{\vee}\right) \in \mathrm{L}^{1}(\mathbb{R}) .
$$

Hence, the claim follows from the Cauchy-Schwarz inequality.

As an immediate corollary we obtain: 
Corollary 3.2.13. Let $\omega \geq 0$, let $v: \mathbb{R} \rightarrow[1, \infty)$ be an admissible weight, and let $f \in \mathrm{W}_{v, \omega}^{2}(\mathbb{R})$. Define $F: \overline{\mathrm{St}}_{\omega} \rightarrow \mathbb{C}$ by setting

$$
F(z):=\int_{\mathbb{R}} f^{\vee}(s) \mathrm{e}^{-\mathrm{i} s z} \mathrm{~d} s
$$

for all $z \in \overline{\mathrm{St}}_{\omega}$. Then $F \in \mathrm{C}_{\mathrm{b}}\left(\overline{\mathrm{St}}_{\omega}\right)$ with

$$
\|F\|_{\infty, \overline{\mathrm{St}}_{\omega}} \leq\left\|\frac{1}{v}\right\|_{2}\|f\|_{v, \omega}
$$

and $F=f$ a.e. on $\mathbb{R}$. Moreover, if $\omega>0$, then $F$ is holomorphic on $\mathrm{St}_{\omega}$.

Now we introduce what we call generalized Sobolev functions.

Definition 3.2.14. Let $\omega>0$, and let $v: \mathbb{R} \rightarrow[1, \infty)$ be an admissible weight. The space

$$
\mathrm{W}_{v}^{2}\left(\overline{\mathrm{St}}_{\omega}\right):=\left\{f \in \mathrm{H}^{\infty}\left(\overline{\mathrm{St}}_{\omega}\right) \mid f_{0} \in \mathrm{W}_{v, \omega}^{2}(\mathbb{R})\right\}
$$

equipped with the norm

$$
\|f\|_{\mathrm{W}_{v}^{2}\left(\overline{\mathrm{St}}_{\omega}\right)}:=\left\|f_{0}\right\|_{v, \omega}
$$

is called the generalized Sobolev space on $\overline{\mathrm{St}}_{\omega}$ with respect to the weight $v$. We abbreviate

$$
\mathrm{W}^{\alpha, 2}\left(\overline{\mathrm{St}}_{\omega}\right):=\mathrm{W}_{v}^{2}\left(\overline{\mathrm{St}}_{\omega}\right)
$$

where $\alpha>\frac{1}{2}$ and $v=(1+|\mathbf{s}|)^{\alpha}$. Moreover, set

$$
\mathrm{W}_{v}^{2}(\mathbb{R}):=\left\{f \in \mathrm{C}_{\mathrm{b}}\left(\overline{\mathrm{St}}_{\omega}\right) \mid f \in \mathrm{W}_{v, 0}^{2}(\mathbb{R})\right\}
$$

and

$$
\|f\|_{\mathrm{W}_{v}^{2}(\mathbb{R})}:=\|f\|_{v, 0}
$$

for $f \in \mathrm{W}_{v}^{2}(\mathbb{R})$. By abuse of notation, we also write

$$
\mathcal{K}_{\omega, v}: \mathrm{W}_{v}^{2}\left(\overline{\mathrm{St}}_{\omega}\right) \rightarrow \mathrm{L}^{2}(\mathbb{R}), \quad f \mapsto v \mathrm{e}^{\omega|\mathbf{s}|} f^{\vee} .
$$

Remarks 3.2.15. Let $\omega \geq 0$, and let $v: \mathbb{R} \rightarrow[1, \infty)$ be an admissible weight.

1) The norm $\|\cdot\|_{\mathrm{W}_{v}^{2}\left(\overline{\mathrm{St}}_{\omega}\right)}$ is well defined. To see this, note that the mapping $\mathrm{W}_{v}^{2}\left(\overline{\mathrm{St}}_{\omega}\right) \rightarrow$ $\mathrm{W}_{v, 0}^{2}(\mathbb{R}), f \mapsto f_{0}$, is injective. Indeed, if $\omega=0$, this is trivial. If $\omega>0$, then this is a consequence of the identity theorem for holomorphic functions.

2) The natural analogue of Lemma 3.2.3 is true for the generalized Sobolev spaces $\mathrm{W}_{v}^{2}\left(\overline{\mathrm{St}}_{\omega}\right)$. Moreover, as $\mathcal{K}_{\omega, v}$ clearly is an isometric isomorphism, $\mathrm{W}_{v}^{2}\left(\overline{\mathrm{St}}_{\omega}\right)$ is a separable Hilbert space.

3) By Corollary 3.2.13, the embedding $\mathrm{W}_{v}^{2}\left(\overline{\mathrm{St}}_{\omega}\right) \hookrightarrow \mathrm{C}_{\mathrm{b}}\left(\overline{\mathrm{St}}_{\omega}\right)$ via the inclusion mapping is continuous.

4) One even has $\mathrm{W}_{v}^{2}(\mathbb{R}) \subseteq \mathrm{C}_{0}(\mathbb{R})$ by the Riemann-Lebesgue lemma. Later we will see that $\mathrm{W}_{v}^{2}\left(\overline{\mathrm{St}}_{\omega}\right) \hookrightarrow \mathrm{C}_{0}\left(\overline{\mathrm{St}}_{\omega}\right)$ for all $\omega \geq 0$ (see Corollary 3.2 .22 . 
Proposition 3.2.16. Let $\omega>0$, and let $v: \mathbb{R} \rightarrow[1, \infty)$ be an admissible weight. Then the mapping

$$
T_{\mathrm{W}}: \mathrm{W}_{v}^{2}\left(\overline{\mathrm{St}}_{\omega}\right) \rightarrow \mathrm{H}^{\infty}\left(\overline{\mathrm{St}}_{\omega}\right), \quad T_{\mathrm{W}} f:=f,
$$

is an isomorphism between the Banach spaces $\mathrm{W}_{v}^{2}\left(\overline{\mathrm{St}}_{\omega}\right)$ and

$$
X:=\left\{g \in \mathrm{H}^{\infty}\left(\overline{\mathrm{St}}_{\omega}\right) \mid\|g\|_{X}:=\left\|g_{\omega}\right\|_{\mathrm{W}_{v}^{2}(\mathbb{R})}+\left\|g_{-\omega}\right\|_{\mathrm{W}_{v}^{2}(\mathbb{R})}<\infty\right\} .
$$

Proof. Fix $f \in \mathrm{W}_{v}^{2}\left(\overline{\mathrm{St}}_{\omega}\right)$ and $r \in\{-\omega, \omega\}$. Then,

$$
\begin{gathered}
\left\|f_{r}\right\|_{\mathrm{W}_{v}^{2}(\mathbb{R})}=\left\|v\left(f_{r}\right)^{\vee}\right\|_{2}=\left\|v \mathrm{e}^{r \mathbf{s}} f^{\vee}\right\|_{2} \\
\leq\left\|v \mathrm{e}^{\omega|\mathbf{s}|} f^{\vee}\right\|_{2}=\|f\|_{\mathrm{W}_{v}^{2}\left(\overline{\mathrm{St}_{\omega}}\right)} .
\end{gathered}
$$

Hence, $f \in X$ with

$$
\|f\|_{X} \leq 2\|f\|_{\mathrm{W}_{v}^{2}\left(\overline{\mathrm{St}}_{\omega}\right)} .
$$

Next, fix $g \in X$. As $g_{\omega}, g_{-\omega} \in \mathrm{L}^{2}(\mathbb{R})$, Lemma 3.2 .8 yields that $g_{0} \in \mathrm{L}^{2}(\mathbb{R})$ and that

$$
\mathrm{e}^{\omega|\mathbf{s}|} g^{\vee}=\left(g_{-\omega}\right)^{\vee} \mathbb{1}_{(-\infty, 0)}+\left(g_{\omega}\right)^{\vee} \mathbb{1}_{(0, \infty)} .
$$

Hence,

$$
\left\|g_{0}\right\|_{v, \omega}=\left\|v \mathrm{e}^{\omega|\mathbf{s}|} g^{\vee}\right\|_{2} \leq\left\|v\left(g_{-\omega}\right)^{\vee}\right\|_{2}+\left\|v\left(g_{\omega}\right)^{\vee}\right\|_{2}=\|g\|_{X}<\infty .
$$

Consequently, $g_{0} \in \mathrm{W}_{v, \omega}^{2}(\mathbb{R})$, and therefore $g \in \mathrm{W}_{v}^{2}\left(\overline{\mathrm{St}}_{\omega}\right)$. This concludes the proof.

Remark 3.2.17. Let $\omega>0$, let $v: \mathbb{R} \rightarrow[1, \infty)$ be an admissible weight, and let $f: \overline{\mathrm{St}}_{\omega} \rightarrow \mathbb{C}$ be a function. By Proposition 3.2.16, one has $f \in \mathrm{W}_{v}^{2}\left(\overline{\mathrm{St}}_{\omega}\right)$ if and only if $f \in \mathrm{H}^{\infty}\left(\overline{\mathrm{St}}_{\omega}\right)$ and $f_{r} \in \mathrm{W}_{v}^{2}(\mathbb{R})$ for both $r=-\omega$ and $r=\omega$. This agrees with the intuition behind the sectorial condition (3.0.4) which says that a Hörmander function on a sector is just a holomorphic function whose restrictions to the boundary satisfy classical Hörmander conditions.

As another corollary of Lemma 3.2.12, together with Proposition 3.2.16, one obtains that every generalized Sobolev space is a Banach algebra.

Proposition 3.2.18. Let $\omega \geq 0$, and let $v: \mathbb{R} \rightarrow[1, \infty)$ be an admissible weight. Then $\left(\mathrm{W}_{v}^{2}\left(\overline{\mathrm{St}}_{\omega}\right),\|\cdot\|_{\mathrm{W}_{v}^{2}\left(\overline{\mathrm{St}}_{\omega}\right)}\right)$ is a Banach algebra with respect to pointwise multiplication.

Note that this was already shown by Strichartz in [51] for the case $\omega=0$. We include a proof for convenience.

Proof. By Proposition 3.2.16, we may suppose that $\omega=0$. Fix $f, g \in \mathrm{W}_{v}^{2}(\mathbb{R})$, set $F:=\left|f^{\vee}\right|, F_{v}:=v\left|f^{\vee}\right|$, and define $G$ and $G_{v}$ similarly. For $s \in \mathbb{R}$ we estimate

$$
\begin{aligned}
v(s)\left|(f g)^{\vee}(s)\right| & =v(s)\left|\left(f^{\vee} * g^{\vee}\right)(s)\right| \\
& \leq \int_{\mathbb{R}} v(s)\left|f^{\vee}(s-t)\right|\left|g^{\vee}(t)\right| \mathrm{d} t
\end{aligned}
$$




$$
\begin{aligned}
& \leq M_{v} \int_{\mathbb{R}}(v(s-t)+v(t))\left|f^{\vee}(s-t)\right|\left|g^{\vee}(t)\right| \mathrm{d} t \\
& =M_{v} \int_{\mathbb{R}}\left(F_{v}(s-t) G(t)+F(s-t) G_{v}(t)\right) \mathrm{d} t \\
& =M_{v}\left(F_{v} * G\right)(s)+M_{v}\left(F * G_{v}\right)(s) .
\end{aligned}
$$

By Lemma 3.2.12, both $F$ and $G$ are in $\mathrm{L}^{1}(\mathbb{R})$ while both $F_{v}$ and $G_{v}$ are in $\mathrm{L}^{2}(\mathbb{R})$. Hence,

$$
\begin{aligned}
\|f g\|_{\mathrm{W}_{v}^{2}(\mathbb{R})} & =\left\|v(f g)^{\vee}\right\|_{2} \\
& \leq M_{v}\left(\left\|F_{v} * G\right\|_{2}+\left\|F * G_{v}\right\|_{2}\right) \\
& \leq M_{v}\left(\left\|F_{v}\right\|_{2}\|G\|_{1}+\|F\|_{1}\left\|G_{v}\right\|_{2}\right) \\
& =M_{v}\left(\|f\|_{\mathrm{W}_{v}^{2}(\mathbb{R})}\left\|g^{\vee}\right\|_{1}+\left\|f^{\vee}\right\|_{1}\|g\|_{\mathrm{W}_{v}^{2}(\mathbb{R})}\right) \\
& \leq 2 M_{v}\left\|\frac{1}{v}\right\|_{2}\|f\|_{\mathrm{W}_{v}^{2}(\mathbb{R})}\|g\|_{\mathrm{W}_{v}^{2}(\mathbb{R})},
\end{aligned}
$$

where Lemma 3.2 .12 was used again for the last estimate.

\section{A Density Result for Generalized Sobolev Functions}

Finally, we shall prove that the space $\mathrm{H}_{0}^{\infty}\left(\mathrm{St}_{\theta}\right)$ is dense in $\mathrm{W}_{v}^{2}\left(\overline{\mathrm{St}}_{\omega}\right)$ for each admissible weight $v: \mathbb{R} \rightarrow[1, \infty)$ and $\theta>\omega \geq 0$.

Proposition 3.2.19. Let $\theta>\omega \geq 0$, and let $v: \mathbb{R} \rightarrow[1, \infty)$ be an admissible weight. Then the space $\mathrm{H}_{0}^{\infty}\left(\mathrm{St}_{\theta}\right)$ is dense in $\mathrm{W}_{v}^{2}\left(\overline{\mathrm{St}}_{\omega}\right)$.

Remark 3.2.20. In [35, p. 268], Kriegler and Weis show that the space

$$
K:=\bigcap_{\omega>0}\left\{f \in \mathrm{H}^{\infty}\left(\mathrm{St}_{\omega}\right)|| f \mid \lesssim(1+|\operatorname{Re} \mathbf{z}|)^{-2}, \widehat{f} \text { has compact support }\right\}
$$

is dense in $\mathrm{W}^{\beta, 2}(\mathbb{R})$ for every $\beta>\frac{1}{2}$. As clearly $K \subseteq \bigcap_{\theta>0} \mathcal{E}\left(\mathrm{St}_{\theta}\right)$, Proposition 3.2 .19 can be seen as a refinement of that result.

Proof of Proposition 3.2.19. As the mapping

$$
\mathcal{K}_{\omega, v}^{-1}: \mathrm{L}^{2}(\mathbb{R}) \rightarrow \mathrm{W}_{v}^{2}\left(\overline{\mathrm{St}}_{\omega}\right), \quad g \mapsto \mathcal{F}\left(\frac{\mathrm{e}^{-\omega|\mathbf{s}|}}{v} g\right),
$$

is an isometric isomorphism (see (3.2.7) ), the subspace $\mathcal{F}\left(\mathrm{C}_{\mathrm{c}}(\mathbb{R})\right)$ is dense in $\mathrm{W}_{v}^{2}\left(\overline{\mathrm{St}}_{\omega}\right)$. Hence, we only need to approximate functions in $\mathcal{F}\left(\mathrm{C}_{\mathrm{c}}(\mathbb{R})\right)$. To this end, fix $\eta \in \mathrm{C}_{\mathrm{c}}(\mathbb{R})$ and let $f:=\mathcal{F} \eta$. Note that then $f \in \mathrm{W}_{v}^{2}\left(\overline{\mathrm{St}}_{\theta^{\prime}}\right)$ for all $\theta^{\prime} \geq 0$. In particular, $f \in \mathrm{W}_{v}^{2}\left(\overline{\mathrm{St}}_{\theta}\right)$. Now, let $\theta_{0} \in(\omega, \theta)$, and let $\psi_{n}$ and $\psi_{n, r}$ for $r= \pm \theta_{0}$ be as in Lemma 3.2.6. Then $\left(\psi_{n} f\right)_{n}$ is a sequence in $\mathrm{H}_{0}^{\infty}\left(\mathrm{St}_{\theta}\right)$. As both $f_{\theta_{0}}, f_{-\theta_{0}} \in \mathrm{L}^{2}(\mathbb{R})$,

$$
\left\|f-\psi_{n} f\right\|_{\mathrm{W}_{v}^{2}\left(\overline{\mathrm{St}}_{\omega}\right)} \lesssim \sum_{r= \pm \theta_{0}}\left\|f_{r}-\psi_{n, r} f_{r}\right\|_{2} \stackrel{n \rightarrow \infty}{\longrightarrow} 0
$$


by Lemma 3.2.10 (see also Definition 3.2.14 and Lemma 3.2.6. This concludes the proof.

As a corollary of Proposition 3.2.19 we obtain the strong continuity of the shift group on $\mathrm{W}_{v}^{2}\left(\overline{\mathrm{St}}_{\omega}\right)$.

Corollary 3.2.21. Let $\omega \geq 0$, and let $v: \mathbb{R} \rightarrow[1, \infty)$ be an admissible weight. Then the shift group $\left(\tau_{t}\right)_{t \in \mathbb{R}}$ is a $C_{0}$-group of isometries on $\mathrm{W}_{v}^{2}\left(\overline{\mathrm{St}}_{\omega}\right)$.

Proof. It is clear that each shift operator $\tau_{t}$ is an isometry on $\mathrm{W}_{v}^{2}\left(\overline{\mathrm{St}}_{\omega}\right)$. Furthermore, whenever $f \in \mathrm{H}_{0}^{\infty}\left(\mathrm{St}_{\theta}\right)$ for some $\theta>\theta_{0}>\omega$,

$$
\left\|f-\tau_{t} f\right\|_{\mathrm{W}_{v}^{2}\left(\overline{\mathrm{St}}_{\omega}\right)} \lesssim \sum_{r= \pm \theta_{0}}\left\|f_{r}-\tau_{t} f_{r}\right\|_{2} \stackrel{t \rightarrow 0}{\longrightarrow} 0 .
$$

Hence, Proposition 3.2 .19 yields the claim.

Corollary 3.2.22. Let $\omega \geq 0$, and let $v: \mathbb{R} \rightarrow[1, \infty)$ be an admissible weight. Then $\mathrm{W}_{v}^{2}\left(\overline{\mathrm{St}}_{\omega}\right) \subseteq \mathrm{C}_{0}\left(\overline{\mathrm{St}}_{\omega}\right)$, i.e., for each $f \in \mathrm{W}_{v}^{2}\left(\overline{\mathrm{St}}_{\omega}\right)$ one has

$$
\lim _{z \in \overline{\mathrm{St}}_{\omega},|z| \rightarrow \infty} f(z)=0 .
$$

Proof. Fix $f \in \mathrm{W}_{v}^{2}\left(\overline{\mathrm{St}}_{\omega}\right)$, let $\theta>\omega$, and choose a sequence $\left(f_{n}\right)_{n}$ in $\mathrm{H}_{0}^{\infty}\left(\mathrm{St}_{\theta}\right)$ with $f_{n} \rightarrow f$ in $\mathrm{W}_{v}^{2}\left(\overline{\mathrm{St}}_{\omega}\right)$. Consequently, by Proposition 3.2.16, $f_{n} \rightarrow f$ uniformly on $\overline{\mathrm{St}}_{\omega}$. As $f_{n} \in \mathrm{C}_{0}\left(\overline{\mathrm{St}}_{\omega}\right)$ for each $n \in \mathbb{N}, f \in \mathrm{C}_{0}\left(\overline{\mathrm{St}}_{\omega}\right)$, too.

\subsection{Generalized Hörmander Functions on a Strip}

Recall that for a function $f: \mathbb{R} \rightarrow \mathbb{C}$ the classical Hörmander condition of order $\alpha>\frac{1}{2}$ reads

$$
\sup _{t \in \mathbb{R}}\left\|\tau_{t} \eta \cdot f\right\|_{\mathrm{W}^{\alpha, 2}(\mathbb{R})}<\infty,
$$

where $0 \neq \eta \in \mathrm{C}_{\mathrm{c}}^{\infty}(\mathbb{R})$ may be chosen arbitrarily. That is, if (3.0.6) is satisfied for one non-zero test function, then (3.0.6) holds for every other test function as well. To arrive at the generalized Hörmander condition introduced below, we alter (3.0.6) in two aspects. First, the classical Sobolev spaces $\mathrm{W}^{\alpha, 2}(\mathbb{R})$ are replaced by the generalized spaces $\mathrm{W}_{v}^{2}\left(\overline{\mathrm{St}}_{\omega}\right)$. Second, the arbitrary test function $0 \neq \eta$ is exchanged for an arbitrary holomorphic function $0 \neq \psi \in \mathrm{H}_{0}^{\infty}\left[\mathrm{St}_{\omega}\right]$.

Definition 3.3.1. Let $\omega \geq 0$, and let $v: \mathbb{R} \rightarrow[1, \infty)$ be an admissible weight. A continuous function $f: \overline{\mathrm{St}}_{\omega} \rightarrow \mathbb{C}$ is called a generalized Hörmander function on $\overline{\mathrm{St}}_{\omega}$ with respect to the weight $v$ if there is $0 \neq \psi \in \mathrm{H}_{0}^{\infty}\left[\mathrm{St}_{\omega}\right]$ such that

$$
\tau_{t} \psi \cdot f \in \mathrm{W}_{v}^{2}\left(\overline{\mathrm{St}}_{\omega}\right) \quad \text { for all } \quad t \in \mathbb{R}, \quad \text { and } \quad \sup _{t \in \mathbb{R}}\left\|\tau_{t} \psi \cdot f\right\|_{\mathrm{W}_{v}^{2}\left(\overline{\mathrm{St}}_{\omega}\right)}<\infty
$$


We write $\operatorname{Hör}_{v}^{2}\left(\overline{\mathrm{St}}_{\omega}\right)$ for the space of all generalized Hörmander functions. If $v=(1+|\mathbf{s}|)^{\alpha}$ for some power $\alpha>\frac{1}{2}$, we also write

$$
\operatorname{Hör}^{\alpha, 2}\left(\overline{\mathrm{St}}_{\omega}\right):=\operatorname{Hör}_{v}^{2}\left(\overline{\mathrm{St}}_{\omega}\right) \text {. }
$$

Example 3.3.2. Let $\omega \geq 0$, and let $v: \mathbb{R} \rightarrow[1, \infty)$ be an admissible weight. Then $\mathrm{e}^{-\mathrm{i} s \mathbf{z}} \in \operatorname{Hör}_{v}^{2}\left(\overline{\mathrm{St}}_{\omega}\right)$ for each $s \in \mathbb{R}$.

Proof. Fix $s_{0} \in \mathbb{R}$. Then, by Lemma 3.1.8, one has

$$
\left\|\psi \mathrm{e}^{-\mathrm{i} s_{0} \mathbf{z}}\right\|_{\mathrm{W}_{v}^{2}\left(\overline{\mathrm{St}_{\omega}}\right)}=\left\|v \mathrm{e}^{\omega|\mathbf{s}|} \tau_{s_{0}} \psi^{\vee}\right\|_{2}=\left\|\tau_{-s_{0}} v \cdot \mathrm{e}^{\omega\left|\mathbf{s}+s_{0}\right|} \psi^{\vee}\right\|_{2} \lesssim v v\left(s_{0}\right) \mathrm{e}^{\omega\left|s_{0}\right|}\|\psi\|_{\mathrm{W}_{v}^{2}\left(\overline{\mathrm{St}_{\omega}}\right)}
$$

for every $\psi \in \mathrm{H}_{0}^{\infty}\left[\mathrm{St}_{\omega}\right]$. Hence,

$$
\sup _{t \in \mathbb{R}}\left\|\tau_{t} \psi \cdot \mathrm{e}^{-\mathrm{i} s \mathbf{z}}\right\|_{\mathrm{W}_{v}^{2}\left(\overline{\mathrm{St}}_{\omega}\right)} \lesssim v, \psi v(s) \mathrm{e}^{\omega|s|}<\infty
$$

for each $s \in \mathbb{R}$.

Generalized Sobolev functions on the strip $\overline{\mathrm{St}}_{\omega}$ are bounded, continuous, and even holomorphic on $\mathrm{St}_{\omega}$, if $\omega>0$. Functions in $\mathrm{Hör}_{v}^{2}\left(\overline{\mathrm{St}}_{\omega}\right)$ share the same property.

Lemma 3.3.3. Let $\omega \geq 0$, let $v: \mathbb{R} \rightarrow[1, \infty)$ be an admissible weight, let $f \in \operatorname{Hör}_{v}^{2}\left(\overline{\operatorname{St}}_{\omega}\right)$, and suppose that 3.3.1 holds for $0 \neq \psi \in \mathrm{H}_{0}^{\infty}\left[\mathrm{St}_{\omega}\right]$. Then $f \in \mathrm{C}_{\mathrm{b}}\left(\overline{\mathrm{St}}_{\omega}\right)\left(f \in \mathrm{H}^{\infty}\left(\overline{\mathrm{St}}_{\omega}\right)\right.$ if $\omega>0)$ with

$$
\|f\|_{\infty, \overline{\mathrm{St}}_{\omega}} \lesssim v, \psi \sup _{t \in \mathbb{R}}\left\|\tau_{t} \psi \cdot f\right\|_{\mathrm{W}_{v}^{2}\left(\overline{\mathrm{St}}_{\omega}\right)}
$$

Proof. As $\psi \neq 0$, the identity theorem for holomorphic functions yields the existence of $s \in \mathbb{R}$ and $\varepsilon>0$ such that $\psi(z) \neq 0$ for all $|\operatorname{Re} z-s| \leq \varepsilon$. Without loss of generality we may suppose that $s=0$ (otherwise consider the function $\tau_{s} \psi$ instead). Therefore, whenever $|t-\operatorname{Re} z| \leq \varepsilon$,

$$
f(z)=\frac{1}{\left(\tau_{t} \psi\right)(z)}\left(\tau_{t} \psi \cdot f\right)(z)
$$

Hence,

$$
\|f\|_{\infty, \overline{\mathrm{St}}_{\omega}} \leq \sup _{|\operatorname{Re} z| \leq \varepsilon} \frac{1}{|\psi(z)|} \cdot \sup _{t \in \mathbb{R}}\left\|\tau_{t} \psi \cdot f\right\|_{\infty, \overline{\mathrm{St}}_{\omega}} \lesssim \sup _{t \in \mathbb{R}}\left\|\tau_{t} \psi \cdot f\right\|_{\mathrm{W}_{v}^{2}\left(\overline{\mathrm{St}}_{\omega}\right)}
$$

If $\omega>0$, then $\tau_{t} \psi \cdot f \in \mathrm{H}^{\infty}\left(\overline{\mathrm{St}}_{\omega}\right)$ for all $t \in \mathbb{R}$. Thus it follows from 3.3 .2 that $f$ is holomorphic on $\mathrm{St}_{\omega}$.

\section{Remarks 3.3.4.}

1) In Proposition 3.3 .19 we shall extend Lemma 3.3.3 and show that every function $f \in \operatorname{Hör}_{v}^{2}\left(\overline{\mathrm{St}}_{\omega}\right)$ is even uniformly continuous on $\overline{\mathrm{St}}_{\omega}$. 
2) Alternatively, one could define the space $\operatorname{Hör}_{v}^{2}\left(\overline{\operatorname{St}}_{\omega}\right)$ as follows: A function $f: \mathbb{R} \rightarrow \mathbb{C}$ belongs to $\mathrm{Hör}_{v}^{2}\left(\overline{\mathrm{St}}_{\omega}\right)$ if there is a function $\psi \in \mathrm{H}_{0}^{\infty}\left[\mathrm{St}_{\omega}\right]$ such that $\tau_{t} \psi_{0} \cdot f \in \mathrm{W}_{v, \omega}^{2}(\mathbb{R})$ for all $t \in \mathbb{R}$ and the family $\left(\tau_{t} \psi_{0} \cdot f\right)_{t \in \mathbb{R}}$ is bounded in $\mathrm{W}_{v, \omega}^{2}(\mathbb{R})$. In that case, one can show that there is a function $F \in \mathrm{C}_{\mathrm{b}}\left(\overline{\mathrm{St}}_{\omega}\right)\left(F \in \mathrm{H}^{\infty}\left(\overline{\mathrm{St}}_{\omega}\right)\right.$ if $\left.\omega>0\right)$ such that $f=F$ a.e. on $\mathbb{R}$.

Since the classical Hörmander conditions $(3.0 .2)$ and $(3.0 .6)$ do not depend on the particular choice of the test function $0 \neq \eta \in \mathrm{C}_{\mathrm{c}}^{\infty}\left(\mathbb{R}_{+}\right)$, respectively $0 \neq \eta \in \mathrm{C}_{\mathrm{c}}^{\infty}(\mathbb{R})$, it is natural to ask whether condition (3.3.1) depends on the particular choice of $0 \neq \psi \in$ $\mathrm{H}_{0}^{\infty}\left[\mathrm{St}_{\omega}\right]$.

\section{On the Dependence on $\psi$}

The goal of this subsection is to show that condition 3.3.1 does not depend on the particular choice of $\psi$. The main idea behind the proof is to establish the Calderón-type reproducing formula (3.3.4). We prepare this with the next two lemmata.

Recall from Section 2.1 that for $\theta>\omega>0, z \in \mathrm{St}_{\theta-\omega}$, and $\psi: \mathrm{St}_{\theta} \rightarrow \mathbb{C}$ we write

$$
\left(\tau_{z} \psi\right)(w)=\psi(w-z) \quad\left(w \in \mathrm{St}_{\omega}\right) .
$$

Lemma 3.3.5. Let $\theta>\omega \geq 0$, let $v: \mathbb{R} \rightarrow[1, \infty)$ be an admissible weight, let $f \in$ $\mathrm{Hör}_{v}^{2}\left(\overline{\mathrm{St}}_{\omega}\right)$, and suppose that (3.3.1) is satisfied for $0 \neq \psi \in \mathrm{H}_{0}^{\infty}\left(\mathrm{St}_{\theta}\right)$. Then the following statements hold:

a) The mapping

$$
\mathbb{R} \rightarrow \mathrm{W}_{v}^{2}\left(\overline{\mathrm{St}}_{\omega}\right), \quad t \mapsto \tau_{t} \psi \cdot f,
$$

is weakly continuous.

b) If $\tau_{z} \psi \cdot f \in \mathrm{W}_{v}^{2}\left(\overline{\mathrm{St}}_{\omega}\right)$ for all $z \in \mathrm{St}_{\theta-\omega}$ and

$$
\sup _{z \in \mathrm{St}_{\theta-\omega}}\left\|\tau_{z} \psi \cdot f\right\|_{\mathrm{W}_{v}^{2}\left(\overline{\mathrm{St}}_{\omega}\right)}<\infty
$$

then the mapping

$$
\mathrm{St}_{\theta-\omega} \rightarrow \mathrm{W}_{v}^{2}\left(\overline{\mathrm{St}}_{\omega}\right), \quad z \mapsto \tau_{z} \psi \cdot f
$$

is holomorphic.

Proof. For a), let $\rho:=0$, and for b), let $\rho:=\theta-\omega$. In any case we obtain a mapping

$$
G: \mathrm{St}_{\rho} \rightarrow \mathrm{W}_{v}^{2}\left(\overline{\mathrm{St}}_{\omega}\right), \quad G(z):=\tau_{z} \psi \cdot f .
$$

As the spaces $\mathrm{W}_{v}^{2}\left(\overline{\mathrm{St}}_{\omega}\right)$ and $\mathrm{L}^{2}(\mathbb{R})$ are isomorphic via the unitary $\mathcal{K}_{\omega, v}$ (see 3.2 .7$)$ ), the map $G$ is weakly continuous (holomorphic) if and only if the same is true for $H:=$ $\mathcal{K}_{\omega, v} \circ G$. As $H$ is uniformly bounded on $\mathrm{St}_{\rho}, H$ is weakly continuous (holomorphic) if and only if each scalar function

$$
(H(\mathbf{z}) \mid \eta)_{\mathrm{L}^{2}(\mathbb{R})} \quad(\eta \in D),
$$


is continuous (holomorphic), where $D$ is dense in $\mathrm{L}^{2}(\mathbb{R})$. Here, we take

$$
D:=\left\{\eta \in \mathrm{C}_{\mathrm{c}}^{2}(\mathbb{R}) \mid 0 \notin \operatorname{supp}(\eta)\right\},
$$

and fix $\eta \in D$. Observe that, by Plancherel,

$$
\begin{aligned}
(H(z) \mid \eta)_{\mathrm{L}^{2}(\mathbb{R})} & =\left(v \mathrm{e}^{\omega|\mathbf{s}|} G(z)^{\vee} \mid \eta\right)_{\mathrm{L}^{2}(\mathbb{R})} \\
& =\left(G(z)^{\vee} \mid v \mathrm{e}^{\omega|\mathbf{s}|} \eta\right)_{\mathrm{L}^{2}(\mathbb{R})} \\
& =\frac{1}{2 \pi}\left(G(z) \mid \mathcal{F}\left(v \mathrm{e}^{\omega|\mathbf{s}|} \eta\right)\right)_{\mathrm{L}^{2}(\mathbb{R})} \\
& =\frac{1}{2 \pi}\left(\tau_{z} \psi \mid \bar{f} \cdot \mathcal{F}\left(v \mathrm{e}^{\omega|\mathbf{s}|} \eta\right)\right)_{\mathrm{L}^{2}(\mathbb{R})^{\prime}}
\end{aligned}
$$

By Remark 3.1.10, we may suppose that $v$ is smooth. As $f$ is bounded (Lemma 3.3.3) and $v \mathrm{e}^{\omega|\mathbf{s}|} \eta \in \mathrm{C}_{\mathbf{c}}^{2}(\mathbb{R})$, it follows that $\bar{f} \cdot \mathcal{F}\left(v \mathrm{e}^{\omega|\mathbf{s}|} \eta\right) \in \mathrm{L}^{1}(\mathbb{R})$. It is thus easy to see that $(H(\mathbf{z}) \mid \eta)$ is indeed a continuous function. If $\rho>0$, one readily verifies that $(H(\mathbf{z}) \mid \eta)$ is even holomorphic. As $D$ is dense in $\mathrm{L}^{2}(\mathbb{R})$, the claims follow.

Lemma 3.3.6. Let $\theta \geq \omega \geq 0$, let $v: \mathbb{R} \rightarrow[1, \infty)$ be an admissible weight, and let $\psi, \varphi \in \mathrm{H}_{0}^{\infty}\left[\mathrm{St}_{\theta}\right]$. Then

$$
\sup _{z \in \operatorname{St}_{\theta-\omega}}\left(\int_{\mathbb{R}}\left\|\tau_{t} \psi \cdot \tau_{z} \varphi\right\|_{\mathrm{W}_{v}^{2}\left(\overline{\mathrm{St}_{\omega}}\right)} \mathrm{d} t\right)<\infty
$$

Proof. Let $\theta^{\prime}>\theta$ with $\psi, \varphi \in \mathrm{H}_{0}^{\infty}\left(\mathrm{St}_{\theta^{\prime}}\right)$. Choose $C, c>0$ with $\max \{|\psi|,|\varphi|\} \leq$ $C \mathrm{e}^{-c|\operatorname{Re} z|}$ on $\mathrm{St}_{\theta^{\prime}}$, and fix $t \in \mathbb{R}$ and $z=s-\mathrm{i} \sigma \in \mathrm{St}_{\theta-\omega}$. We show the estimate

$$
\left\|\tau_{t} \psi \cdot \tau_{z} \varphi\right\|_{\mathrm{W}_{v}^{2}\left(\overline{\mathrm{St}}_{\omega}\right)} \lesssim v, \omega, \theta^{\prime} \frac{C^{2}}{\sqrt{c}}(1+2 c|t-s|)^{\frac{1}{2}} \mathrm{e}^{-c|t-s|} .
$$

The claim then follows readily. To establish (3.3.3), first note that

$$
\left\|\tau_{t} \psi \cdot \tau_{z} \varphi\right\|_{\mathrm{W}_{v}^{2}\left(\overline{\mathrm{St}}_{\omega}\right)}=\left\|\tau_{t-s} \psi \cdot \varphi_{\sigma}\right\|_{\mathrm{W}_{v}^{2}\left(\overline{\mathrm{St}}_{\omega}\right)} .
$$

Hence, we may suppose that $s=0$. Moreover, by Lemma 3.2.10 (see also Definition 3.2 .14 ,

$$
\left\|\tau_{t-s} \psi \cdot \varphi_{\sigma}\right\|_{\mathrm{W}_{v}^{2}\left(\overline{\mathrm{St}}_{\omega}\right)} \lesssim v, \omega, \delta \sum_{r= \pm \delta}\left\|\tau_{t} \psi_{r} \cdot \varphi_{\sigma+r}\right\|_{2}
$$

for each $\omega<\delta<\omega+\theta^{\prime}-\theta$. Finally, whenever $r= \pm \delta$,

$$
\left\|\tau_{t} \psi_{r} \cdot \varphi_{r+\sigma}\right\|_{2}^{2} \leq \int_{\mathbb{R}} C^{4} \mathrm{e}^{-2 c|t-u|} \mathrm{e}^{-2 c|u|} \mathrm{d} u=\frac{C^{4}}{2 c}(1+2 c|t|) \mathrm{e}^{-2 c|t|}
$$

by an elementary computation.

For the next lemma we recall the notation

$$
\psi^{*}:=\overline{\psi(\overline{\mathbf{z}})} \quad\left(\psi \in \mathrm{H}_{0}^{\infty}[\mathbb{R}]\right) .
$$


Lemma 3.3.7. Let $\omega \geq 0$, let $v: \mathbb{R} \rightarrow[1, \infty)$ be an admissible weight, let $f \in \operatorname{Hör}_{v}^{2}\left(\overline{\operatorname{St}}_{\omega}\right)$, and suppose that (3.3.1) holds for $0 \neq \psi \in \mathrm{H}_{0}^{\infty}\left[\mathrm{St}_{\omega}\right]$. Then, for every $\varphi \in \mathrm{H}_{0}^{\infty}\left[\mathrm{St}_{\omega}\right]$, the mapping

$$
\mathbb{R} \rightarrow \mathrm{W}_{v}^{2}\left(\overline{\mathrm{St}}_{\omega}\right), \quad r \mapsto\left(\tau_{r} \psi^{*} \cdot \varphi\right)\left(\tau_{r} \psi \cdot f\right),
$$

belongs to $\mathrm{L}^{1}\left(\mathbb{R} ; \mathrm{W}_{v}^{2}\left(\overline{\mathrm{St}}_{\omega}\right)\right)$, and $\varphi f \in \mathrm{W}_{v}^{2}\left(\overline{\mathrm{St}}_{\omega}\right)$ with

$$
\varphi f=\frac{1}{\|\psi\|_{2}^{2}} \int_{\mathbb{R}}\left(\tau_{r} \psi^{*} \cdot \varphi\right)\left(\tau_{r} \psi \cdot f\right) \mathrm{d} r .
$$

In particular,

$$
\|\varphi f\|_{\mathrm{W}_{v}^{2}\left(\overline{\mathrm{St}_{\omega}}\right)} \lesssim v\left(\frac{1}{\|\psi\|_{2}^{2}} \int_{\mathbb{R}}\left\|\tau_{r} \psi^{*} \cdot \varphi\right\|_{\mathrm{W}_{v}^{2}\left(\overline{\mathrm{St}_{\omega}}\right)} \mathrm{d} r\right) \sup _{t \in \mathbb{R}}\left\|\tau_{t} \psi \cdot f\right\|_{\mathrm{W}_{v}^{2}\left(\overline{\mathrm{St}}_{\omega}\right)}
$$

Proof. Fix $\varphi \in \mathrm{H}_{0}^{\infty}\left[\mathrm{St}_{\omega}\right]$ and set

$$
G: \mathbb{R} \rightarrow \mathrm{W}_{v}^{2}\left(\overline{\mathrm{St}}_{\omega}\right), \quad G(r):=\left(\tau_{r} \psi^{*} \cdot \varphi\right)\left(\tau_{r} \psi \cdot f\right) .
$$

Then $G$ is well-defined. By Lemma 3.3.5 a) and Corollary 3.2.21, $G$ is weakly continuous. As $\mathrm{W}_{v}^{2}\left(\overline{\mathrm{St}}_{\omega}\right)$ is separable, Pettis's measurability theorem yields that $G$ is strongly measurable. Finally, note that

$$
\|G(r)\|_{\mathrm{W}_{v}^{2}\left(\overline{\mathrm{St}}_{\omega}\right)} \lesssim\left\|\tau_{r} \psi \cdot f\right\|_{\mathrm{W}_{v}^{2}\left(\overline{\mathrm{St}}_{\omega}\right)}\left\|\tau_{r} \psi^{*} \cdot \varphi\right\|_{\mathrm{W}_{v}^{2}\left(\overline{\mathrm{St}_{\omega}}\right)}
$$

for each $r \in \mathbb{R}$. Hence, by Lemma 3.3.6 and 3.3.1), $G \in \mathrm{L}^{1}\left(\mathbb{R} ; \mathrm{W}_{v}^{2}\left(\overline{\mathrm{St}}_{\omega}\right)\right)$. Next, we show (3.3.4). For this we may suppose that

$$
\|\psi\|_{2}^{2}=\int_{\mathbb{R}} \psi(s-r) \psi^{*}(s-r) \mathrm{d} r=1 \quad(s \in \mathbb{R}) .
$$

As $\mathrm{W}_{v}^{2}\left(\overline{\mathrm{St}}_{\omega}\right)$ canonically embeds into $\mathrm{C}_{\mathrm{b}}\left(\overline{\mathrm{St}}_{\omega}\right)$, the integral

$$
\int_{\mathbb{R}} G(r) \mathrm{d} r
$$

converges also in $\mathrm{C}_{\mathrm{b}}\left(\overline{\mathrm{St}}_{\omega}\right)$. Hence,

$$
\begin{aligned}
\left(\int_{\mathbb{R}} G(r) \mathrm{d} r\right)(s) & =\int_{\mathbb{R}}\left(\tau_{r} \psi \cdot \tau_{r} \psi^{*} \cdot \varphi f\right)(s) \mathrm{d} r \\
& =\int_{\mathbb{R}} \psi(s-r) \psi^{*}(s-r) \varphi(s) f(s) \mathrm{d} s \\
& =\varphi(s) f(s)
\end{aligned}
$$

for all $s \in \mathbb{R}$, where 3.3 .6 was used in the last line. Therefore

$$
\varphi f=\int_{\mathbb{R}} \tau_{r} \psi \cdot \tau_{r} \psi^{*} \cdot \varphi f \mathrm{~d} r
$$

as elements of $\mathrm{C}_{\mathrm{b}}\left(\overline{\mathrm{St}}_{\omega}\right)$. However, as the right hand side is in $\mathrm{W}_{v}^{2}\left(\overline{\mathrm{St}}_{\omega}\right), \varphi f \in \mathrm{W}_{v}^{2}\left(\overline{\mathrm{St}}_{\omega}\right)$, too, and the equality carries over to $\mathrm{W}_{v}^{2}\left(\overline{\mathrm{St}}_{\omega}\right)$. The estimate 3.3.5 follows from 3.3.4 and the triangle inequality. 
As a corollary of Lemma 3.3 .7 we get that the generalized Hörmander condition (3.3.1) does not depend on the particular choice of $\psi$.

Corollary 3.3.8. Let $\omega \geq 0$, let $v: \mathbb{R} \rightarrow[1, \infty)$ be an admissible weight, let $f \in$ $\operatorname{Hör}_{v}^{2}\left(\overline{\mathrm{St}}_{\omega}\right)$, and suppose that condition 3.3.1 holds for $0 \neq \psi \in \mathrm{H}_{0}^{\infty}\left[\mathrm{St}_{\omega}\right]$. Then, for every $\varphi \in \mathrm{H}_{0}^{\infty}\left[\mathrm{St}_{\omega}\right]$ and $t \in \mathbb{R}$ one has $\tau_{t} \varphi \cdot f \in \mathrm{W}_{v}^{2}\left(\overline{\mathrm{St}}_{\omega}\right)$, and

$$
\sup _{t \in \mathbb{R}}\left\|\tau_{t} \varphi \cdot f\right\|_{\mathrm{W}_{v}^{2}\left(\overline{\mathrm{St}}_{\omega}\right)} \lesssim v\left(\frac{1}{\|\psi\|_{2}^{2}} \int_{\mathbb{R}}\left\|\tau_{r} \psi^{*} \cdot \varphi\right\|_{\mathrm{W}_{v}^{2}\left(\overline{\mathrm{St}}_{\omega}\right)} \mathrm{d} r\right) \sup _{t \in \mathbb{R}}\left\|\tau_{t} \psi \cdot f\right\|_{\mathrm{W}_{v}^{2}\left(\overline{\mathrm{St}}_{\omega}\right)} .
$$

Remark 3.3.9. The idea to look for integral representations like (3.3.4) is not new. In fact, Calderón reproducing formulas are a classical tool in the study of function spaces (see e.g., [16, Chapter 1 - 3, Chapter 5 - 6]).

We are now in a position to define a reasonable norm on $\operatorname{Hör}_{v}^{2}\left(\overline{\operatorname{St}}_{\omega}\right)$.

Definition 3.3.10. Let $\omega \geq 0$, and let $v: \mathbb{R} \rightarrow[1, \infty)$ be an admissible weight. For $f \in \operatorname{Hör}_{v}^{2}\left(\overline{\mathrm{St}}_{\omega}\right)$ we set

$$
\|f\|_{\mathrm{Hör}_{v}^{2}\left(\overline{\mathrm{St}_{\omega}}\right)}:=\sup _{t \in \mathbb{R}}\left\|\tau_{t} \psi \cdot f\right\|_{\mathrm{W}_{v}^{2}\left(\overline{\mathrm{St}}_{\omega}\right)}
$$

\section{Remarks 3.3.11.}

1) The map $f \mapsto\|f\|_{\mathrm{Hör}_{v}^{2}\left(\overline{\mathrm{St}}_{\omega}\right)}$ clearly is a norm. Moreover, as a consequence of Corollary 3.3.8, replacing $\psi$ with any other function $0 \neq \psi \in \mathrm{H}_{0}^{\infty}\left[\mathrm{St}_{\omega}\right]$ in the definition above leads to an equivalent norm.

2) By Example 3.3.2, one has

$$
\left\|\mathrm{e}^{-\mathrm{i} s \mathbf{z}}\right\|_{\mathrm{Hör}_{v}^{2}\left(\overline{\mathrm{St}}_{\omega}\right)} \lesssim v v(s) \mathrm{e}^{\omega|s|}
$$

for all $s \in \mathbb{R}$.

Each generalized Hörmander space is a Banach algebra.

Proposition 3.3.12. Let $\omega \geq 0$, and let $v: \mathbb{R} \rightarrow[1, \infty)$ be an admissible weight. Then $\left(\mathrm{Hör}_{v}^{2}\left(\overline{\mathrm{St}}_{\omega}\right),\|\cdot\|_{\mathrm{Hör}_{v}^{2}\left(\overline{\mathrm{St}}_{\omega}\right)}\right)$ is a Banach algebra with respect to pointwise multiplication.

Proof. For the proof of completeness fix a Cauchy sequence $\left(f_{n}\right)_{n}$ in $\operatorname{Hör}_{v}^{2}\left(\overline{\operatorname{St}}_{\omega}\right)$. As $\mathrm{Hör}_{v}^{2}\left(\overline{\mathrm{St}}_{\omega}\right)$ continuously embeds into $\mathrm{C}_{\mathrm{b}}\left(\overline{\mathrm{St}}_{\omega}\right)$ by Lemma 3.3.3. $\left(f_{n}\right)_{n}$ is also a Cauchy sequence in $\mathrm{C}_{\mathrm{b}}\left(\overline{\mathrm{St}}_{\omega}\right)$. Hence, there is $f \in \mathrm{C}_{\mathrm{b}}\left(\overline{\mathrm{St}}_{\omega}\right)$ with

$$
\left\|f_{n}-f\right\|_{\infty, \overline{\mathrm{St}}_{\omega}} \stackrel{n \rightarrow \infty}{\longrightarrow} 0 .
$$

Consequently, for fixed $n \in \mathbb{N}$ and $\psi \in \mathrm{H}_{0}^{\infty}\left[\mathrm{St}_{\omega}\right]$ one has $\psi\left(f_{n}-f_{m}\right) \rightarrow \psi\left(f_{n}-f\right)$ in $\mathrm{L}^{1}(\mathbb{R})$ and therefore $\left(\psi\left(f_{n}-f_{m}\right)\right)^{\vee} \rightarrow\left(\psi\left(f_{n}-f\right)\right)^{\vee}$ uniformly on $\mathbb{R}$ as $m \rightarrow \infty$. Hence, by Fatou's lemma,

$$
\left\|\psi\left(f_{n}-f\right)\right\|_{\mathrm{W}_{v}^{2}\left(\overline{\mathrm{St}}_{\omega}\right)} \leq \liminf _{m \rightarrow \infty}\left\|\psi\left(f_{n}-f_{m}\right)\right\|_{\mathrm{W}_{v}^{2}\left(\overline{\mathrm{St}}_{\omega}\right)} \leq \sup _{m \geq n}\left\|\psi\left(f_{n}-f_{m}\right)\right\|_{\mathrm{W}_{v}^{2}\left(\overline{\mathrm{St}_{\omega}}\right)}
$$


for every $n \in \mathbb{N}$. From this one concludes that $f_{n}-f \in \operatorname{Hör}_{v}^{2}\left(\overline{\operatorname{St}}_{\omega}\right)$, whence $f \in \operatorname{Hör}_{v}^{2}\left(\overline{\operatorname{St}}_{\omega}\right)$, and that

$$
\left\|f_{n}-f\right\|_{\operatorname{Hör}_{v}^{2}\left(\overline{\mathrm{St}}_{\omega}\right)} \leq \sup _{m \geq n}\left\|f_{n}-f_{m}\right\|_{\operatorname{Hör}_{v}^{2}\left(\overline{\mathrm{St}_{\omega}}\right)} .
$$

This yields completeness. For the submultiplicativity of the norm fix $f, g \in \operatorname{Hör}_{v}^{2}\left(\overline{\operatorname{St}}_{\omega}\right)$ and note that

$$
\begin{aligned}
\sup _{t \in \mathbb{R}}\left\|\tau_{t} \psi^{2} \cdot f g\right\|_{\mathrm{W}_{v}^{2}\left(\overline{\mathrm{St}_{\omega}}\right)} & =\sup _{t \in \mathbb{R}}\left\|\left(\tau_{t} \psi \cdot f\right)\left(\tau_{t} \psi \cdot g\right)\right\|_{\mathrm{W}_{v}^{2}\left(\overline{\mathrm{St}}_{\omega}\right)} \\
& \lesssim v\|f\|_{\operatorname{Hör}_{v}^{2}\left(\overline{\mathrm{St}_{\omega}}\right)}\|g\|_{\operatorname{Hör}_{v}^{2}\left(\overline{\mathrm{St}_{\omega}}\right)} .
\end{aligned}
$$

Hence, $f g \in \operatorname{Hör}_{v}^{2}\left(\overline{\mathrm{St}}_{\omega}\right)$ and $\|f g\|_{\mathrm{Hör}_{v}^{2}\left(\overline{\mathrm{St}}_{\omega}\right)} \lesssim\|f\|_{\operatorname{Hör}_{v}^{2}\left(\overline{\mathrm{St}}_{\omega}\right)}\|g\|_{\mathrm{Hör}_{v}^{2}\left(\overline{\mathrm{St}}_{\omega}\right)}$.

There is also another way to see that for each $s \in \mathbb{R}$ the function $\mathrm{e}^{-\mathrm{i} s \mathbf{z}}$ belongs to $\operatorname{Hör}_{v}^{2}\left(\overline{\mathrm{St}}_{\omega}\right)$. Namely, whenever $f \in \mathrm{H}^{\infty}\left(\mathrm{St}_{\theta}\right)$ for $\theta>\omega$, one already has that $f \in$ $\operatorname{Hör}_{v}^{2}\left(\overline{\mathrm{St}}_{\omega}\right)$ by the next proposition.

Proposition 3.3.13. Let $\theta>\omega \geq 0$, and let $v: \mathbb{R} \rightarrow[1, \infty)$ be an admissible weight. Then $\left.f\right|_{\overline{\mathrm{St}}_{\omega}} \in \mathrm{Hör}_{v}^{2}\left(\overline{\mathrm{St}}_{\omega}\right)$ for all $f \in \mathrm{H}^{\infty}\left(\mathrm{St}_{\theta}\right)$. Moreover, the embedding $\mathrm{H}^{\infty}\left(\mathrm{St}_{\theta}\right) \hookrightarrow$ $\mathrm{Hör}_{v}^{2}\left(\overline{\mathrm{St}}_{\omega}\right)$ is continuous.

Proof. Fix $f \in \mathrm{H}^{\infty}\left(\mathrm{St}_{\theta}\right)$, and let $\theta_{0} \in(\omega, \theta)$. For each $t \in \mathbb{R}$ one has

$$
\left\|\tau_{t} \psi \cdot f\right\|_{\mathrm{W}_{v}^{2}\left(\overline{\mathrm{St}}_{\omega}\right)} \lesssim_{v, \theta_{0}, \omega} \sum_{r= \pm \theta_{0}}\left\|\tau_{t} \psi_{r} \cdot f_{r}\right\|_{2} \leq\left(\left\|\psi_{-\theta_{0}}\right\|_{2}+\left\|\psi_{\theta_{0}}\right\|_{2}\right)\|f\|_{\infty, \overline{\mathrm{St}}_{\theta}}
$$

by Lemma 3.2.10 (see also Definition 3.2.14). This yields the claim.

\section{Connection to Classical Hörmander Spaces}

Now we compare the classical Hörmander condition (3.0.6) to condition 3.3.1) in the case $\omega=0$.

Lemma 3.3.14. Let $v: \mathbb{R} \rightarrow[1, \infty)$ be an admissible weight, let $0 \neq \psi \in \mathrm{H}_{0}^{\infty}[\mathbb{R}]$, and let $0 \neq \eta \in \mathrm{C}_{\mathrm{c}}^{\infty}(\mathbb{R})$. Then there is a constant $c=c(v, \eta, \psi)>0$ such that

$$
\frac{1}{c} \sup _{t \in \mathbb{R}}\left\|\tau_{t} \eta \cdot f\right\|_{\mathrm{W}_{v}^{2}(\mathbb{R})} \leq \sup _{t \in \mathbb{R}}\left\|\tau_{t} \psi \cdot f\right\|_{\mathrm{W}_{v}^{2}(\mathbb{R})} \leq c \sup _{t \in \mathbb{R}}\left\|\tau_{t} \eta \cdot f\right\|_{\mathrm{W}_{v}^{2}(\mathbb{R})}
$$

for all $f \in \mathrm{C}_{\mathrm{b}}(\mathbb{R})$.

Proof. Fix $f \in \mathrm{C}_{\mathrm{b}}(\mathbb{R})$. For the left inequality, suppose that

$$
\sup _{t \in \mathbb{R}}\left\|\tau_{t} \psi \cdot f\right\|_{\mathrm{W}_{v}^{2}(\mathbb{R})}<\infty .
$$

Then, by Corollary 3.3.8, we may further suppose that $\psi=\psi$. In that case one has

$$
\left\|\tau_{t} \eta \cdot f\right\|_{\mathrm{W}_{v}^{2}(\mathbb{R})}=\left\|\tau_{t}\left(\frac{\eta}{\psi}\right) \cdot \tau_{t} \psi \cdot f\right\|_{\mathrm{W}_{v}^{2}(\mathbb{R})} \lesssim v\left\|\frac{\eta}{\psi}\right\|_{\mathrm{W}_{v}^{2}(\mathbb{R})}\left\|\tau_{t} \psi \cdot f\right\|_{\mathrm{W}_{v}^{2}(\mathbb{R})}
$$




$$
\leq\left\|\frac{\eta}{\psi}\right\|_{\mathrm{W}_{v}^{2}(\mathbb{R})} \cdot \sup _{s \in \mathbb{R}}\left\|\tau_{t} \psi \cdot f\right\|_{\mathrm{W}_{v}^{2}(\mathbb{R})} .
$$

As $\mathrm{C}_{\mathrm{c}}^{\infty}(\mathbb{R}) \subseteq \mathrm{W}_{v}^{2}(\mathbb{R})$ (Corollary 3.2.4, see also Definition 3.2.14, $\left\|\frac{\eta}{\psi}\right\|_{\mathrm{W}_{v}^{2}(\mathbb{R})}<\infty$, and the left inequality in $(3.3 .8)$ can be concluded. For the right inequality, suppose that

$$
\sup _{t \in \mathbb{R}}\left\|\tau_{t} \eta \cdot f\right\|_{\mathrm{W}_{v}^{2}(\mathbb{R})}<\infty .
$$

On one hand, since $0 \neq \eta$, by multiplying with a suitable constant we may suppose that

$$
\int_{\mathbb{R}} \eta(s-r) \bar{\eta}(s-r) \mathrm{d} r=1 \quad(s \in \mathbb{R}) .
$$

On the other hand, there are constants $c_{0}, c_{1}>0$ (depending on $v, \psi$ and $\eta$ ) such that

$$
\left\|\tau_{t} \bar{\eta} \cdot \tau_{s} \psi\right\|_{\mathrm{W}_{v}^{2}(\mathbb{R})} \leq c_{0} \mathrm{e}^{-c_{1}|t-s|} \quad(s, t \in \mathbb{R}) .
$$

Indeed, by Lemma 3.1.7, there is $N \in \mathbb{N}$ such that

$$
v \lesssim(1+|\mathbf{s}|)^{N}
$$

Moreover, there is a constant $c_{1}>0$ with

$$
\left|\psi^{(k)}\right| \lesssim \mathrm{e}^{-c_{1}|\operatorname{Re} \mathbf{z}|} \quad(0 \leq k \leq N) .
$$

Hence, for each $t \in \mathbb{R}$ one has

$$
\begin{aligned}
\left\|\tau_{-t} \bar{\eta} \cdot \psi\right\|_{\mathrm{W}_{v}^{2}(\mathbb{R})} & =\left\|\bar{\eta} \cdot \tau_{t} \psi\right\|_{\mathrm{W}_{v}^{2}(\mathbb{R})} \lesssim\left\|\bar{\eta} \cdot \tau_{t} \psi\right\|_{\mathrm{W}^{N, 2}(\mathbb{R})} \\
& \approx \sum_{k=0}^{N}\left\|\bar{\eta}^{(k)} \cdot \tau_{t} \psi^{(N-k)}\right\|_{2} \\
& \lesssim \sup _{r \in \operatorname{supp}(\eta)}\left|\psi^{(N-k)}(r-t)\right| \\
& \lesssim \sup _{r \in \operatorname{supp}(\eta)}\left(\mathrm{e}^{-c_{1}|r-t|}\right) \\
& \leq \sup _{r \in \operatorname{supp}(\eta)}\left(\mathrm{e}^{c_{1}|r|}\right) \cdot \mathrm{e}^{-c_{1}|t|} .
\end{aligned}
$$

This yields (3.3.10). Now, using both (3.3.9) and 3.3.10 one can argue as in the proof of Lemma 3.3 .7 to arrive at the estimate

$$
\sup _{t \in \mathbb{R}}\left\|\tau_{t} \psi \cdot f\right\|_{\mathrm{W}_{v}^{2}(\mathbb{R})} \lesssim\left(\int_{\mathbb{R}}\left\|\tau_{r} \bar{\eta} \cdot \psi\right\|_{\mathrm{W}_{v}^{2}(\mathbb{R})} \mathrm{d} r\right) \sup _{s \in \mathbb{R}}\left\|\tau_{s} \eta \cdot f\right\|_{\mathrm{W}_{v}^{2}(\mathbb{R})} \lesssim \sup _{s \in \mathbb{R}}\left\|\tau_{s} \eta \cdot f\right\|_{\mathrm{W}_{v}^{2}(\mathbb{R})} .
$$

Remark 3.3.15. By Lemma 3.3.14, if one replaces test functions $\eta \in \mathrm{C}_{\mathrm{c}}^{\infty}(\mathbb{R})$ with holomorphic functions $\psi \in \mathrm{H}_{0}^{\infty}[\mathbb{R}]$ in the classical Hörmander condition (3.0.6), then one obtains the same function space. This justifies our shift from test functions to $\mathrm{H}_{0}^{\infty}$-functions and shows that condition (3.3.1) does indeed generalize condition 3.0.6). 
The next proposition (together with the previous lemma) recovers the strip type condition (3.0.7). That is, if $\omega>0$, a function $f: \overline{\mathrm{St}}_{\omega} \rightarrow \mathbb{C}$ belongs to $\operatorname{Hör}_{v}^{2}\left(\overline{\mathrm{St}}_{\omega}\right)$ if and only if $f \in \mathrm{H}^{\infty}\left(\overline{\mathrm{St}}_{\omega}\right)$ and the restrictions $f_{ \pm \omega}$ belong to $\mathrm{Hör}_{v}^{2}(\mathbb{R})$. This is the analogue of Proposition 3.2.16 for Hörmander functions.

Proposition 3.3.16. Let $\omega>0$, and let $v: \mathbb{R} \rightarrow[1, \infty)$ be an admissible weight. Then $\mathrm{Hör}_{v}^{2}\left(\overline{\mathrm{St}}_{\omega}\right)$ is isomorphic to

$$
X:=\left\{g \in \mathrm{H}^{\infty}\left(\overline{\mathrm{St}}_{\omega}\right) \mid\|g\|_{X}:=\left\|g_{\omega}\right\|_{\operatorname{Hör}_{v}^{2}(\mathbb{R})}+\left\|g_{-\omega}\right\|_{\operatorname{Hör}_{v}^{2}(\mathbb{R})}<\infty\right\}
$$

via the map

$$
T_{\mathrm{H}}: \operatorname{Hör}_{v}^{2}\left(\overline{\mathrm{St}}_{\omega}\right) \rightarrow X, \quad T_{\mathrm{H}} f:=f .
$$

Proof. Let $f \in \operatorname{Hör}_{v}^{2}\left(\overline{\mathrm{St}}_{\omega}\right)$. Then $f \in \mathrm{H}^{\infty}\left(\overline{\mathrm{St}}_{\omega}\right)$ by Lemma 3.3.2. By Proposition 3.2.16.

$$
\left\|\tau_{t} \psi \cdot f\right\|_{\mathrm{W}_{v}^{2}\left(\overline{\mathrm{St}}_{\omega}\right)} \approx \sum_{r= \pm \omega}\left\|\tau_{t} \psi_{r} \cdot f_{r}\right\|_{\mathrm{W}_{v}^{2}(\mathbb{R})}
$$

for every $t \in \mathbb{R}$, hence $f_{ \pm \omega} \in \operatorname{Hör}_{v}^{2}(\mathbb{R})$ and

$$
\|f\|_{X} \lesssim\|f\|_{\operatorname{Hör}_{v}^{2}\left(\overline{\mathrm{St}}_{\omega}\right)}<\infty .
$$

Therefore, $T_{\mathrm{H}}$ is well defined and bounded. Next, fix $g \in X$. Note that for every $t \in \mathbb{R}$ and $r \in\{-\omega, \omega\}$ one has $\tau_{t} \psi_{r} \cdot g_{r} \in \mathrm{W}_{v}^{2}(\mathbb{R})$. Hence, by Proposition 3.2.16. $\tau_{t} \psi \cdot g \in \mathrm{W}_{v}^{2}\left(\overline{\mathrm{St}}_{\omega}\right)$ with

$$
\left\|\tau_{t} \psi \cdot g\right\|_{\mathrm{W}_{v}^{2}\left(\overline{\mathrm{St}}_{\omega}\right)} \approx \sum_{r= \pm \omega}\left\|\tau_{t} \psi_{r} \cdot f_{r}\right\|_{\mathrm{W}_{v}^{2}(\mathbb{R})} .
$$

Consequently, $g \in \operatorname{Hör}_{v}^{2}\left(\overline{\mathrm{St}}_{\omega}\right)$ with $\|g\|_{\mathrm{Hör}_{v}^{2}\left(\overline{\mathrm{St}}_{\omega}\right)} \lesssim\|g\|_{X}$. Therefore, $T_{\mathrm{H}}$ is bijective. This yields the claim.

\section{Hörmander Functions are Uniformly Continuous}

Next, we extend Lemma 3.3.3 by showing that each generalized Hörmander function is uniformly continuous on $\overline{\mathrm{St}}_{\omega}$. First, note the following corollary of Lemma 3.3.5.b), Lemma 3.3.6 and Lemma 3.3.7.

Corollary 3.3.17. Let $\theta>\omega \geq 0$, let $v: \mathbb{R} \rightarrow[1, \infty)$ be an admissible weight, let $f \in \operatorname{Hör}_{v}^{2}\left(\overline{\mathrm{St}}_{\omega}\right)$, and let $\psi \in \mathrm{H}_{0}^{\infty}\left[\mathrm{St}_{\theta}\right]$. Then the mapping

$$
\mathrm{St}_{\theta-\omega} \rightarrow \mathrm{W}_{v}^{2}\left(\overline{\mathrm{St}}_{\omega}\right), \quad z \mapsto \tau_{z} \psi \cdot f,
$$

is well defined and belongs to $\mathrm{H}^{\infty}\left(\mathrm{St}_{\theta-\omega} ; \mathrm{W}_{v}^{2}\left(\overline{\mathrm{St}}_{\omega}\right)\right)$.

Proof. Fix $z \in \mathrm{St}_{\theta-\omega}$. By Lemma 3.3.7, $\tau_{z} \psi \cdot f \in \mathrm{W}_{v}^{2}\left(\overline{\mathrm{St}}_{\omega}\right)$. By the estimate 3.3.5,

$$
\left\|\tau_{z} \psi \cdot f\right\|_{\mathrm{W}_{v}^{2}\left(\overline{\mathrm{St}_{\omega}}\right)} \lesssim v\left(\frac{1}{\|\psi\|_{2}^{2}} \int_{\mathbb{R}}\left\|\tau_{r} \psi^{*} \cdot \tau_{z} \psi\right\|_{\mathrm{W}_{v}^{2}\left(\overline{\mathrm{St}}_{\omega}\right)} \mathrm{d} r\right)\|f\|_{\mathrm{Hör}_{v}^{2}\left(\overline{\mathrm{St}_{\omega}}\right)},
$$

where the right hand side is uniformly bounded in $z \in \mathrm{St}_{\theta-\omega}$ by Lemma 3.3.6. Hence, Lemma 3.3.5.b) yields the claim. 
Also note the following representation formula for generalized Hörmander functions.

Lemma 3.3.18. Let $\omega \geq 0$, let $v: \mathbb{R} \rightarrow[1, \infty)$ be an admissible weight, and let $f \in$ $\operatorname{Hör}_{v}^{2}\left(\overline{\mathrm{St}}_{\omega}\right)$. Then, for each $z \in \overline{\mathrm{St}}_{\omega}$,

$$
f(z)=\int_{\mathbb{R}}\left(\tau_{z} \psi \cdot f\right)^{\vee}(s) \mathrm{e}^{-\mathrm{i} s z} \mathrm{~d} s .
$$

Proof. For each $u \in \mathbb{C}, \tau_{u} \psi \cdot f$ is a Sobolev function and therefore admits the representation

$$
\left(\tau_{u} \psi \cdot f\right)(z)=\int_{\mathbb{R}}\left(\tau_{u} \psi \cdot f\right)^{\vee}(s) \mathrm{e}^{-\mathrm{i} s z} \mathrm{~d} s \quad\left(z \in \overline{\mathrm{St}}_{\omega}\right)
$$

by Corollary 3.2 .13 . Hence,

$$
f(z)=\psi(0) f(z)=\left(\tau_{z} \psi \cdot f\right)(z)=\int_{\mathbb{R}}\left(\tau_{z} \psi \cdot f\right)^{\vee}(s) \mathrm{e}^{-\mathrm{i} s z} \mathrm{~d} s
$$

for each $z \in \overline{\mathrm{St}}_{\omega}$.

Proposition 3.3.19. Let $\omega \geq 0$, let $v: \mathbb{R} \rightarrow[1, \infty)$ be an admissible weight, and let $f \in \operatorname{Hör}_{v}^{2}\left(\overline{\mathrm{St}}_{\omega}\right)$. Then $f$ is uniformly continuous on $\overline{\mathrm{St}}_{\omega}$. Moreover, the embedding $\mathrm{Hör}_{v}^{2}\left(\overline{\mathrm{St}}_{\omega}\right) \hookrightarrow \mathrm{UC}_{\mathrm{b}}\left(\overline{\mathrm{St}}_{\omega}\right)$ is continuous.

Proof. By Corollary 3.3.17, the map

$$
\Psi_{f}: \mathbb{C} \rightarrow \mathrm{W}_{v}^{2}\left(\overline{\mathrm{St}}_{\omega}\right), \quad \Psi_{f}(z):=\tau_{z} \psi \cdot f,
$$

is well defined, entire, and bounded on each strip $\mathrm{St}_{\delta}, \delta>0$. In particular, $\Psi_{f}$ is uniformly continuous on $\overline{\mathrm{St}}_{\omega}$. By (3.3.11),

$$
\begin{aligned}
f(z) & =\int_{\mathbb{R}}\left(\tau_{z} \psi \cdot f\right)^{\vee}(s) \mathrm{e}^{-\mathrm{i} s z} \mathrm{~d} s \\
& =\int_{\mathbb{R}} v(s) \mathrm{e}^{-\omega|s|}\left(\tau_{z} \psi \cdot f\right)^{\vee}(s) \cdot \frac{\mathrm{e}^{-\omega|s|}}{v(s)} \mathrm{e}^{-\mathrm{i} s z} \mathrm{~d} s \\
& =\left(\mathcal{K}_{\omega, v} \Psi_{f}(z) \mid \frac{\mathrm{e}^{-\omega|s|}}{v} \mathrm{e}^{-\mathrm{i} s z}\right)_{\mathrm{L}^{2}(\mathbb{R})}
\end{aligned}
$$

for each $z \in \overline{\mathrm{St}}_{\omega}$. As $\mathcal{K}_{\omega, v}: \mathrm{W}_{v}^{2}\left(\overline{\mathrm{St}}_{\omega}\right) \rightarrow \mathrm{L}^{2}(\mathbb{R})$ (see 3.2 .7 ) is an isometric isomorphism, $\mathcal{K}_{\omega, v} \circ \Psi_{f}$ is uniformly continuous as well. Hence, once we verify that

$$
H: \overline{\mathrm{St}}_{\omega} \rightarrow \mathrm{L}^{2}(\mathbb{R}), \quad H(z):=\frac{\mathrm{e}^{-\omega|\mathbf{s}|}}{v} \mathrm{e}^{-\mathrm{i} \mathbf{s} z},
$$

is uniformly continuous on $\overline{\mathrm{St}}_{\omega}$, it follows that $f \in \mathrm{UC}_{\mathrm{b}}\left(\overline{\mathrm{St}}_{\omega}\right)$. To this end, let $z, u \in \overline{\mathrm{St}}_{\omega}$ and observe that

$$
\|H(z)-H(u)\|_{2}^{2}=\int_{\mathbb{R}} \frac{\mathrm{e}^{-2 \omega|s|}}{v(s)^{2}}\left|\mathrm{e}^{-\mathrm{i} s z}-\mathrm{e}^{-\mathrm{i} s u}\right|^{2} \mathrm{~d} s \stackrel{|z-u| \rightarrow 0}{\longrightarrow} 0
$$

by the dominated convergence theorem. Therefore, $H$ is indeed uniformly continuous. For the rest of the claim, recall that by Lemma 3.3.3 the embedding $\operatorname{Hör}_{v}^{2}\left(\overline{\operatorname{St}}_{\omega}\right) \hookrightarrow$ $\mathrm{C}_{\mathrm{b}}\left(\overline{\mathrm{St}}_{\omega}\right)$ is continuous. 
Remark 3.3.20. In view of Proposition 3.3.19, it is natural to ask whether the shift group $\left(\tau_{s}\right)_{s \in \mathbb{R}}$ is strongly continuous on $\operatorname{Hör}_{v}^{2}\left(\overline{\mathrm{St}}_{\omega}\right)$. In general, this is not the case as the next example shows. Let $\omega=0$, let $v=1+|\mathbf{s}|$, and let $\eta \in \mathrm{C}_{\mathrm{c}}^{\infty}(\mathbb{R})$ with $\left.\eta\right|_{[-1,1]}=1$. Then, by Lemma 3.3.14.

$$
\|g\|_{\mathrm{Hör}^{1,2}(\mathbb{R})} \approx \sup _{R \in \mathbb{R}}\left\|\tau_{R} \eta \cdot g\right\|_{\mathrm{W}^{1,2}(\mathbb{R})} \gtrsim \sup _{R \in \mathbb{R}}\left(\int_{R-1}^{R+1}\left|g^{\prime}(s)\right|^{2} \mathrm{~d} s\right)^{\frac{1}{2}}
$$

for all $g \in \operatorname{Hör}^{1,2}(\mathbb{R})$. Define

$$
f: \mathbb{R} \rightarrow \mathbb{C}, \quad f(t):=\int_{0}^{t} \mathrm{e}^{\mathrm{i} s^{2}} \mathrm{~d} s .
$$

It is well known that the limit

$$
\lim _{t \rightarrow \infty} f(t)=\int_{0}^{\infty} \mathrm{e}^{\mathrm{i} s^{2}} \mathrm{~d} s
$$

exists (this is the Fresnel integral, see [45, (7.8)] for example). As $f \in \mathrm{C}_{\mathrm{b}}^{1}(\mathbb{R})$, one has $f \in \operatorname{Hör}^{1,2}(\mathbb{R})$ as well. However, using 3.3 .12 , we have for each $t>0$

$$
\begin{aligned}
\left\|\tau_{t} f-f\right\|_{\mathrm{Hö} 1,2(\mathbb{R})}^{2} & \gtrsim \sup _{R \in \mathbb{R}} \int_{R-1}^{R+1}\left|f^{\prime}(s-t)-f^{\prime}(s)\right|^{2} \mathrm{~d} s \\
& =\sup _{R \in \mathbb{R}} \int_{R-1}^{R+1}\left|\mathrm{e}^{\mathrm{i}(s-t)^{2}}-\mathrm{e}^{\mathrm{i} s^{2}}\right|^{2} \mathrm{~d} s \\
& =\sup _{R \in \mathbb{R}} \int_{R-1}^{R+1}\left|\mathrm{e}^{-2 \mathrm{i} s t} \mathrm{e}^{\mathrm{i} t^{2}}-1\right|^{2} \mathrm{~d} s \\
& \geq \int_{\frac{\pi}{2 t}-1}^{\frac{\pi}{2 t}+1}\left|\mathrm{e}^{-2 \mathrm{i} s t} \mathrm{e}^{\mathrm{i} t^{2}}-1\right|^{2} \mathrm{~d} s \\
& =\frac{1}{2 t} \int_{\pi-2 t}^{\pi+2 t}\left|\mathrm{e}^{-\mathrm{i} s} \mathrm{e}^{\mathrm{i} t^{2}}-1\right|^{2} \mathrm{~d} s \\
& \stackrel{t \searrow 0}{\longrightarrow} 4 .
\end{aligned}
$$

Hence, $\tau_{t} f$ does not converge to $f$ in $\operatorname{Hör}^{1,2}(\mathbb{R})$ as $t \searrow 0$.

\section{An Important Inequality}

We conclude Section 3.3 with an important inequality which plays a key role in the proof of our main result (Theorem 4.3.1).

Theorem 3.3.21. Let $\theta \geq \omega \geq 0$, let $v: \mathbb{R} \rightarrow[1, \infty)$ be an admissible weight, and let $\psi \in \mathrm{H}_{0}^{\infty}\left[\mathrm{St}_{\theta}\right]$. Then

$$
\sup _{s \in \mathbb{R}, z \in \operatorname{St}_{\theta-\omega}}\left|v(s) \mathrm{e}^{\omega|s|}\left(\tau_{z} \psi f\right)^{\vee}(s)\right| \lesssim v, \psi\|f\|_{\operatorname{Horr}_{v}^{2}\left(\overline{\operatorname{St}}_{\omega}\right)}
$$

for each $f \in \operatorname{Hör}_{v}^{2}\left(\overline{\mathrm{St}}_{\omega}\right)$. 
Proof. Fix $z \in \mathrm{St}_{\theta-\omega}$ and define

$$
G_{z}: \mathbb{R} \rightarrow \mathrm{L}^{1}(\mathbb{R}), \quad G_{z}(r):=\left(\tau_{r} \psi^{*} \cdot \tau_{z} \psi\right)\left(\tau_{r} \psi \cdot f\right)=\left(\tau_{r} \psi^{*} \cdot \tau_{r} \psi\right)\left(\tau_{z} \psi \cdot f\right) .
$$

Then $G_{z}$ is a well-defined element of $\mathrm{L}^{1}\left(\mathbb{R} ; \mathrm{L}^{1}(\mathbb{R})\right)$. To see this, just note that whenever $g \in \mathrm{L}^{1}(\mathbb{R})$ and $h \in \mathrm{L}^{\infty}(\mathbb{R})$ one has $\tau_{\mathbf{s}} g \cdot h \in \mathrm{L}^{1}\left(\mathbb{R} ; \mathrm{L}^{1}(\mathbb{R})\right)$. Indeed, by the strong continuity of the shift group on $\mathrm{L}^{1}(\mathbb{R})$, the mapping $\tau_{\mathbf{s}} g \cdot h$ is continuous and thus measurable, and one has

$$
\begin{aligned}
\int_{\mathbb{R}}\left\|\tau_{r} g \cdot h\right\|_{1} \mathrm{~d} r & =\int_{\mathbb{R}} \int_{\mathbb{R}}|g(s-r)||h(s)| \mathrm{d} s \mathrm{~d} r \\
& =\int_{\mathbb{R}} \int_{\mathbb{R}}|g(s-r)||h(s)| \mathrm{d} r \mathrm{~d} s=\|g\|_{1}\|h\|_{1}<\infty .
\end{aligned}
$$

Next, observe that

$$
\tau_{z} \psi \cdot f=\frac{1}{\|\psi\|_{2}^{2}} \int_{\mathbb{R}} G_{z}(r) \mathrm{d} r=\frac{1}{\pi} \int_{\mathbb{R}}\left(\tau_{r} \psi^{*} \cdot \tau_{z} \psi\right)\left(\tau_{r} \psi \cdot f\right) \mathrm{d} r
$$

in $\mathrm{L}^{1}(\mathbb{R})$. Hence, since $\mathcal{F}^{-1} \in \mathcal{L}\left(\mathrm{L}^{1}(\mathbb{R}) ; \mathrm{C}_{0}(\mathbb{R})\right)$,

$$
\left(\tau_{z} \psi \cdot f\right)^{\vee}(s)=\frac{1}{\pi} \int_{\mathbb{R}}\left(\tau_{r} \psi \cdot \tau_{r} \psi^{*} \cdot \tau_{z} \psi \cdot f\right)^{\vee}(s) \mathrm{d} r
$$

for every $s \in \mathbb{R}$. Now choose $c \geq 0$ such that $v(s+t) \leq c v(s) v(t)$ for all $s, t \in \mathbb{R}$ (see Lemma 3.1.8). Note that for each $g, h \in \mathrm{L}^{2}\left(\mathbb{R}, v \mathrm{e}^{\omega|\mathbf{s}|} \mathrm{d} s\right)$ one has

$$
\begin{aligned}
v(s) \mathrm{e}^{\omega|s|}|(g * h)(s)| & \leq \int_{\mathbb{R}} v(s) \mathrm{e}^{\omega|s|}|g(t-s) h(t)| \mathrm{d} t \\
& \leq c \int_{\mathbb{R}}\left(v(t-s) \mathrm{e}^{\omega|t-s|}|g(t-s)|\right) \cdot\left(v(t) \mathrm{e}^{\omega|t|}|h(t)|\right) \mathrm{d} t \\
& \leq c\left\|v \mathrm{e}^{\omega|\mathbf{s}|} g\right\|_{2}\left\|v \mathrm{e}^{\omega|\mathbf{s}|} h\right\|_{2} .
\end{aligned}
$$

Therefore, using (3.3.13), we may estimate

$$
\begin{aligned}
\left|v(s) \mathrm{e}^{\omega|s|}\left(\tau_{z} \psi \cdot f\right)^{\vee}(s)\right| & \leq \frac{1}{\pi} \int_{\mathbb{R}} v(s) \mathrm{e}^{\omega|s|}\left|\left(\tau_{r} \psi \cdot \tau_{r} \psi^{*} \cdot \tau_{z} \psi \cdot f\right)^{\vee}(s)\right| \mathrm{d} r \\
& =\frac{1}{\pi} \int_{\mathbb{R}} v(s) \mathrm{e}^{\omega|s|}\left|\left(\tau_{r} \psi^{*} \cdot \tau_{z} \psi\right)^{\vee} *\left(\tau_{r} \psi \cdot f\right)^{\vee}\right|(s) \mathrm{d} r \\
& \leq \frac{c}{\pi} \int_{\mathbb{R}}\left\|\tau_{r} \psi^{*} \cdot \tau_{z} \psi\right\|_{\mathrm{W}_{v}^{2}(\mathbb{R})}\left\|\tau_{r} \psi \cdot f\right\|_{\mathrm{W}_{v}^{2}(\mathbb{R})} \mathrm{d} r \\
& \leq \frac{c}{\pi}\left(\int_{\mathbb{R}}\left\|\tau_{r} \psi^{*} \cdot \tau_{z} \psi\right\|_{\mathrm{W}_{v}^{2}(\mathbb{R})} \mathrm{d} r\right)\|f\|_{\operatorname{Hör}_{v}^{2}\left(\overline{\operatorname{St}}_{\omega}\right)}
\end{aligned}
$$

for each $s \in \mathbb{R}$ and $z \in \mathrm{St}_{\theta-\omega}$. As

$$
\sup _{z \in \operatorname{St}_{\theta-\omega}}\left(\int_{\mathbb{R}}\left\|\tau_{r} \psi \cdot \tau_{z} \psi\right\|_{\mathrm{W}_{v}^{2}\left(\overline{\mathrm{St}}_{\omega}\right)} \mathrm{d} r\right)<\infty
$$

by Lemma 3.3.6, the claim follows. 
Remark 3.3.22. A sectorial version of Theorem 3.3 .21 for polynomial weights and very special $\psi$ is given by [6, Lemma 6]. That version appears implicitly in the proofs of [41, Theorem 4] and [17, Theorem 2.2].

\subsection{Generalized Hörmander Functions on a Sector}

We finally arrive at Hörmander functions on sectors.

Definition 3.4.1. For $\omega \in[0, \pi)$ write

$$
\overline{\mathrm{S}}_{\omega}^{*}:=\overline{\mathrm{S}}_{\omega} \backslash\{0\} .
$$

Let $v: \mathbb{R} \rightarrow[1, \infty)$ be an admissible weight. The space

$$
\mathrm{W}_{v}^{2}\left(\overline{\mathrm{S}}_{\omega}^{*}\right):=\left\{f \in \mathrm{C}_{\mathrm{b}}\left(\overline{\mathrm{S}}_{\omega}^{*}\right) \mid f \circ \mathrm{e}^{\mathbf{z}} \in \mathrm{W}_{v}^{2}\left(\overline{\mathrm{St}}_{\omega}\right)\right\}
$$

equipped with the norm

$$
\|f\|_{\mathrm{W}_{v}^{2}\left(\overline{\mathrm{S}}_{\omega}^{*}\right)}:=\left\|f \circ \mathrm{e}^{\mathbf{z}}\right\|_{\mathrm{W}_{v}^{2}\left(\overline{\mathrm{St}_{\omega}}\right)}
$$

is called the generalized Sobolev space on $\mathrm{S}_{\omega}$ with respect to $v$. The space

$$
\operatorname{Hör}_{v}^{2}\left(\overline{\mathrm{S}}_{\omega}^{*}\right):=\left\{f: \overline{\mathrm{S}}_{\omega}^{*} \rightarrow \mathbb{C} \mid f \circ \mathrm{e}^{\mathbf{z}} \in \operatorname{Hör}_{v}^{2}\left(\overline{\operatorname{St}}_{\omega}\right)\right\}
$$

with the norm

$$
\|f\|_{\mathrm{Hör}_{v}^{2}\left(\overline{\mathrm{S}}_{\omega}^{*}\right)}:=\left\|f \circ \mathrm{e}^{\mathbf{z}}\right\|_{\operatorname{Hör}_{v}^{2}\left(\overline{\mathrm{St}}_{\omega}\right)}
$$

is called the generalized Hörmander space on $\mathrm{S}_{\omega}$ with respect to $v$. We abbreviate

$$
\mathrm{W}^{\alpha, 2}\left(\overline{\mathrm{S}}_{\omega}^{*}\right):=\mathrm{W}_{v}^{2}\left(\overline{\mathrm{S}}_{\omega}^{*}\right), \quad \text { and } \quad \operatorname{Hör}^{\alpha, 2}\left(\overline{\mathrm{S}}_{\omega}^{*}\right):=\operatorname{Hör}_{v}^{2}\left(\overline{\mathrm{S}}_{\omega}^{*}\right) \text {, }
$$

where $\alpha>\frac{1}{2}$ and $v=(1+|\mathbf{s}|)^{\alpha}$.

The key properties of the spaces $\mathrm{W}_{v}^{2}\left(\overline{\mathrm{S}}_{\omega}^{*}\right)$ and $\operatorname{Hör}_{v}^{2}\left(\overline{\mathrm{S}}_{\omega}^{*}\right)$ are collected in the next two propositions. As each statement follows directly from its strip counterpart, we omit the proofs. (Recall from Remark 2.4.3 that $f \in \mathcal{E}\left(\mathrm{S}_{\theta}\right)$, resp. $f \in \mathrm{H}_{0}^{\infty}\left(\mathrm{S}_{\theta}\right)$, if and only if $f \circ \mathrm{e}^{\mathbf{z}} \in \mathcal{E}\left(\mathrm{St}_{\theta}\right)$, resp. $f \circ \mathrm{e}^{\mathbf{z}} \in \mathrm{H}_{0}^{\infty}\left(\mathrm{St}_{\theta}\right)$.)

Proposition 3.4.2. Let $0 \leq \omega<\theta<\pi$, and let $v: \mathbb{R} \rightarrow[1, \infty)$ be an admissible weight. Then the following statements hold:

a) One has $\mathcal{E}\left[\mathrm{S}_{\omega}\right] \subseteq \mathrm{W}_{v}^{2}\left(\overline{\mathrm{S}}_{\omega}^{*}\right)$, and the subspace $\mathrm{H}_{0}^{\infty}\left(\mathrm{S}_{\theta}\right)$ is dense in $\mathrm{W}_{v}^{2}\left(\overline{\mathrm{S}}_{\omega}^{*}\right)$.

b) The canonical embeddings

$$
\mathrm{W}_{v}^{2}\left(\overline{\mathrm{S}}_{\omega}^{*}\right) \hookrightarrow \mathrm{C}_{0}\left(\overline{\mathrm{S}}_{\omega}\right), \quad \operatorname{Hör}_{v}^{2}\left(\overline{\mathrm{S}}_{\omega}^{*}\right) \hookrightarrow \mathrm{C}_{\mathrm{b}}\left(\overline{\mathrm{S}}_{\omega}^{*}\right), \quad \text { and } \quad \mathrm{H}^{\infty}\left(\mathrm{S}_{\theta}\right) \hookrightarrow \operatorname{Hör}_{v}^{2}\left(\overline{\mathrm{S}}_{\omega}^{*}\right)
$$

are continuous.

Proposition 3.4.3. Let $\omega \in[0, \pi)$, let $v: \mathbb{R} \rightarrow[1, \infty)$ be an admissible weight, and let $\mathrm{F} \in\left\{\mathrm{W}_{v}^{2}, \mathrm{Hör}_{v}^{2}\right\}$. Then the following statements hold:

a) The space $\left(\mathrm{F}\left(\overline{\mathrm{S}}_{\omega}^{*}\right),\|\cdot\|_{\mathrm{F}\left(\overline{\mathrm{S}}_{\omega}^{*}\right)}\right)$ is a Banach algebra with respect to pointwise multiplication.

b) If $\omega>0$, then $f \in \mathrm{F}\left(\overline{\mathrm{S}}_{\omega}^{*}\right)$ if and only if $f \in \mathrm{H}^{\infty}\left(\mathrm{S}_{\omega}\right) \cap \mathrm{C}_{\mathrm{b}}\left(\overline{\mathrm{S}}_{\omega}^{*}\right)$ and $f\left(\mathrm{e}^{\mathrm{\pm i} \omega} \mathrm{s}\right) \in \mathrm{F}\left(\mathbb{R}_{+}\right)$. 


\section{Connection to Classical Hörmander Spaces}

Let $\alpha>\frac{1}{2}$, and let $\omega>0$. By Proposition 3.4.3. b) and Lemma 3.3.14, a function $f: \overline{\mathrm{S}}_{\omega}^{*} \rightarrow \mathbb{C}$ belongs to the space Hör ${ }^{\alpha, 2}\left(\overline{\mathrm{S}}_{\omega}^{*}\right)$ if and only if $f$ is holomorphic and bounded on the sector $\mathrm{S}_{\omega}$ and both restrictions $f\left(\mathrm{e}^{ \pm \mathrm{i} \omega} \mathrm{e}^{\mathrm{s}}\right)$ satisfy the condition (3.0.6), i.e.,

$$
\sup _{t \in \mathbb{R}}\left\|\tau_{t} \eta \cdot f\left(\mathrm{e}^{ \pm \mathrm{i} \omega} \mathrm{e}^{\mathbf{s}}\right)\right\|_{\mathrm{W}^{\alpha, 2}(\mathbb{R})}<\infty
$$

where $0 \neq \eta \in \mathrm{C}_{\mathrm{c}}^{\infty}(\mathbb{R})$ is arbitrary. As this is equivalent to $f\left(\mathrm{e}^{ \pm \mathrm{i} \omega} \mathbf{s}\right)$ satisfying the classical Hörmander condition 3.0 .3 (cf. [32, (4.13)]), it follows that $f \in \operatorname{Hör}^{\alpha, 2}\left(\overline{\mathrm{S}}_{\omega}^{*}\right)$ if and only if $f$ satisfies the sectorial Hörmander condition (3.0.4). Hence, the spaces $\mathrm{Hör}^{\alpha, 2}\left(\overline{\mathrm{S}}_{\omega}^{*}\right)$ coincide with the spaces $\mathrm{H}^{\infty}\left(\mathrm{S}_{\omega} ; \alpha\right)$ considered in [17], [39], [47], and [6]. 


\section{Chapter 4}

\section{The Holomorphic Hörmander Functional Calculus}

The goal of this chapter is to establish a Hörmander calculus for strip type and sectorial operators based on the classical holomorphic calculus. More precisely, given a sectorial operator $A$ with angle of sectoriality $\omega \in[0, \pi)$, for example, we want to find a reasonable extension of the holomorphic calculus of $A$ which covers all expressions

$$
f(A) \quad\left(f \in \operatorname{Hör}_{v}^{2}\left(\overline{\mathrm{S}}_{\omega}^{*}\right)\right),
$$

that is, the angle $\omega$ may be chosen sharp. This is not feasible by means of the holomorphic calculus alone.

The basic idea behind our construction is to consider a subclass of strip type and sectorial operators for which the elementary holomorphic calculus can be extended to a bounded Sobolev calculus. Recall that any Hörmander function may be algebraically regularized to a Sobolev function by multiplication with an $\mathrm{H}_{0}^{\infty}$-function. Hence, once a bounded Sobolev calculus is established, a Hörmander calculus is obtained by the classical algebraic extension procedure.

This idea goes back to Kriegler and Weis who considered the case where one may only establish an unbounded Sobolev calculus for 0-sectorial operators and operators of strip type 0 (cf. [32], [35, and [36]). It should be mentioned that Kriegler and Weis do not work with the algebraic extension procedure but rather follow a more "hands-on" approach to define their functional calculus.

Chapter 4 consists of four sections. As we did in Chapter 3 , we concentrate on the strip case first and discuss the (extended) Sobolev calculus of strip type operators in Section 4.1. Amongst other things, a convergence lemma for the Hörmander functional calculus is presented (see Theorem 4.1.14).

As the setting of Section 4.1 is actually more restrictive (in the case of strip type 0 ) than the one considered by Kriegler and Weis, Section 4.2 is dedicated to the discussion 
on how one may extend Kriegler's and Weis's methods to non-zero strip type operators. Here we generalize the results of Section 4.1. However, the purpose of Section 4.2 is only an informative one as the contents are not used in the subsequent part of the text.

In Section 4.3 , we discuss conditions for strip type operators that lead to a bounded (or even $\gamma$-bounded) Hörmander calculus. In particular, the main result of this thesis is presented in the form of Theorem 4.3.1.

Finally, Section 4.4 deals with the question of how to define a Hörmander calculus for sectorial operators and how results from the strip case may be carried over to the sectorial case. The answer to the second question is given in form of a composition rule for the respective calculi (Theorem 4.4.3). We also give a sectorial version of our main result (Theorem 4.4.4).

\subsection{The Extended Sobolev Functional Calculus}

Let $A$ be a strip type operator of strip type $\omega \geq 0$. We want to extend the holomorphic calculus of $A$ in such a way that also expressions $f(A)$ are covered where the function $f$ may only be defined on the closed strip $\overline{\mathrm{St}}_{\omega}$.

The difficulty lies in the fact that $f$ may not be "reachable" within the holomorphic calculus. The operator $f(A)$ is only defined in the holomorphic calculus of $A$ if $f$ can be algebraically regularized to a function in $\mathcal{E}\left[\mathrm{St}_{\omega}\right]$. However, if $f$ cannot be suitably extended to a larger strip $\mathrm{St}_{\theta}$, then there is no non-trivial function $e \in \mathcal{E}\left[\mathrm{St}_{\omega}\right]$ with ef $\in \mathcal{E}\left[\mathrm{St}_{\omega}\right]$. Hence, this "domain gap" cannot by bridged by algebraic means alone.

The answer to this problem lies in looking for a topological solution instead of an algebraic one. Recall that for each $\theta>\omega$ and each admissible weight $v: \mathbb{R} \rightarrow[1, \infty)$ the space $\mathrm{H}_{0}^{\infty}\left(\mathrm{St}_{\theta}\right)$ is dense in $\mathrm{W}_{v}^{2}\left(\overline{\mathrm{St}}_{\omega}\right)$. Hence, one may consider all those strip type operators whose elementary calculus on $\mathrm{H}_{0}^{\infty}\left(\mathrm{St}_{\theta}\right)$ can be topologically extended to the algebra $\mathrm{W}_{v}^{2}\left(\overline{\mathrm{St}}_{\omega}\right)$.

\section{The Bounded Sobolev Calculus}

Throughout the entire Section 4.1, the following class of operators is of interest.

Definition 4.1.1. Let $A$ be a strip type operator on a Banach space $X$ and let $\omega \geq$ $\omega_{\text {st }}(A)$. We say that $A$ has a bounded $\mathrm{W}_{v}^{2}\left(\overline{\mathrm{St}}_{\omega}\right)$-calculus if there is $\theta>\omega$ and a constant $c \geq 0$ such that

$$
\|\psi(A)\| \leq c\|\psi\|_{\mathrm{W}_{v}^{2}\left(\overline{\mathrm{St}_{\omega}}\right)}
$$

for all $\psi \in \mathrm{H}_{0}^{\infty}\left(\mathrm{St}_{\theta}\right)$.

Suppose that $A$ is a strip type operator given as in Definition 4.1.1. Then, by Proposition 3.2 .19 , one can extend the elementary holomorphic calculus of $A$ to a bounded algebra homomorphism

$$
\Phi: \mathrm{W}_{v}^{2}\left(\overline{\mathrm{St}}_{\omega}\right) \rightarrow \mathcal{L}(X) .
$$


The next lemma shows that $\Phi$ extends the elementary holomorphic calculus of $A$ in a natural way.

Lemma 4.1.2. Let $A$ and $\Phi$ be given as above, and let $f \in \mathcal{E}\left[\mathrm{St}_{\omega}\right]$. Then

$$
\Phi(f)=f(A) .
$$

Proof. Let $\theta>\omega$ be given as in Definition 4.1.1, and let $\theta_{0} \in(\omega, \theta)$ such that $f \in \mathcal{E}\left[\mathrm{St}_{\theta_{0}}\right]$. Then $f \in \mathrm{W}_{v}^{2}\left(\overline{\mathrm{St}}_{\theta_{0}}\right)$ as well. Hence, there is a sequence $\left(\psi_{n}\right)_{n}$ in $\mathrm{H}_{0}^{\infty}\left(\mathrm{St}_{\theta}\right)$ with $\psi_{n} \rightarrow f$ in $\mathrm{W}_{v}^{2}\left(\overline{\mathrm{St}}_{\theta_{0}}\right)$. In this case, $\psi_{n} \rightarrow f$ in $\mathrm{W}_{v}^{2}\left(\overline{\mathrm{St}}_{\omega}\right)$ as well, whence

$$
\Phi(f)=\lim _{n \rightarrow \infty} \Phi\left(\psi_{n}\right)=\lim _{n \rightarrow \infty} \psi_{n}(A)
$$

in $\mathcal{L}(X)$. Now, let $|\operatorname{Im} \lambda|>\omega$. Noting that $\psi_{n} \rightarrow f$ also uniformly on $\overline{\operatorname{St}}_{\theta_{0}}$ by Corollary 3.2 .13 and writing the following operators as Cauchy integrals, one obtains

$$
\begin{aligned}
R(\lambda, A)^{2} f(A) & =\left(\frac{f}{(\lambda-\mathbf{z})^{2}}\right)(A)=\lim _{n \rightarrow \infty}\left(\frac{\psi_{n}}{(\lambda-\mathbf{z})^{2}}\right)(A) \\
& =\lim _{n \rightarrow \infty} R(\lambda, A)^{2} \psi_{n}(A)=R(\lambda, A)^{2} \Phi(f) .
\end{aligned}
$$

As $R(\lambda, A)^{2}$ is injective, $\Phi(f)=f(A)$.

The Sobolev functional calculus appears naturally in the context of $C_{0}$-groups.

Examples 4.1.3. Let $-\mathrm{i} A$ generate a $C_{0}$-group $\left(U_{s}\right)_{s \in \mathbb{R}}$ on a Banach space $X$.

1) Suppose that $\left(U_{s}\right)_{s \in \mathbb{R}}$ is bounded, i.e. $M:=\sup _{s \in \mathbb{R}}\left\|U_{s}\right\|<\infty$. Then $A$ has a bounded $\mathrm{W}_{v}^{2}(\mathbb{R})$-calculus for each admissible weight $v: \mathbb{R} \rightarrow[1, \infty)$.

Proof. Let $v: \mathbb{R} \rightarrow[1, \infty)$ be an admissible weight, and let $\psi \in \mathcal{E}[\mathbb{R}]$. By Proposition 2.5.14,

$$
\psi(A)=\int_{\mathbb{R}} \psi^{\vee}(s) U_{s} \mathrm{~d} s=\int_{\mathbb{R}}\left(v(s) \psi^{\vee}(s)\right) \cdot\left(\frac{1}{v(s)} U_{s}\right) \mathrm{d} s,
$$

hence

$$
\|\psi(A)\| \leq M\left\|\frac{1}{v}\right\|_{2}\left\|v \psi^{\vee}\right\|_{2}=M\left\|\frac{1}{v}\right\|_{2}\|\psi\|_{\mathrm{W}_{v}^{2}(\mathbb{R})}
$$

by the Cauchy-Schwarz inequality.

2) More generally, suppose that there is $\omega \geq 0$ and a measurable function $v_{U}: \mathbb{R} \rightarrow$ $[0, \infty)$ with

$$
\left\|U_{s}\right\| \leq v_{U}(s) \mathrm{e}^{\omega|s|} \quad(s \in \mathbb{R}) .
$$

Then, $A$ has a bounded $\mathrm{W}_{v}^{2}\left(\overline{\mathrm{St}}_{\omega}\right)$-calculus for every admissible weight with $\frac{v_{U}}{v} \in$ $\mathrm{L}^{2}(\mathbb{R})$. This can be proven similarly to the example above. However, this is also a consequence of Proposition 4.1.6 below. 
Remark 4.1.4. Let $-\mathrm{i} A$ generate a $C_{0}$-group $\left(U_{s}\right)_{s \in \mathbb{R}}$. By Example 4.1.3 2$), A$ has a bounded $\mathrm{W}_{v}^{2}\left(\overline{\mathrm{St}}_{\omega}\right)$-calculus for every $\omega>\theta(U)$ (see Definition 2.5 .3 for $\theta(U)$ ) and every admissible weight $v: \mathbb{R} \rightarrow[1, \infty)$. Therefore, the most interesting question is whether there is an admissible weight $v$ such that $A$ also admits a bounded $\mathrm{W}_{v}^{2}\left(\overline{\operatorname{St}}_{\omega}\right)$-calculus for $\omega=\theta(U)$. To this end, Example 4.1.3.2) suggests to consider the function

$$
v_{U}(s):=\mathrm{e}^{-\theta(U)|s|}\left\|U_{s}\right\| \quad(s \in \mathbb{R}),
$$

and to look for admissible weights $v$ with the property that $\frac{v_{U}}{v} \in \mathrm{L}^{2}(\mathbb{R})$. Such a weight need not exist as the next example shows.

Example 4.1.5. Let $w: \mathbb{R} \rightarrow[1, \infty)$ be a continuous function such that

$$
w(s+t) \leq w(s) w(t) \quad(s, t \in \mathbb{R}) .
$$

Consider the shift group $\left(\tau_{s}\right)_{s \in \mathbb{R}}$ on the weighted space $\mathrm{L}^{p}(\mathbb{R}, w(s) \mathrm{d} s)$ for $1 \leq p<\infty$. Then

$$
\left\|\tau_{s}\right\|=w(s)^{\frac{1}{p}} \quad(s \in \mathbb{R}) .
$$

Proof. Fix $s \in \mathbb{R}$. Clearly, $\left\|\tau_{s}\right\| \leq w(s)^{\frac{1}{p}}$. For the reverse inequality, set $h_{\varepsilon}:=$ $(2 \varepsilon)^{-\frac{1}{p}} \mathbb{1}_{[-\varepsilon, \varepsilon]}$ for $\varepsilon>0$ and observe that

$$
\left\|\tau_{s} h_{\varepsilon}\right\|_{\mathrm{L}^{p}(\mathbb{R}, w(s) \mathrm{d} s)}^{p} \stackrel{\varepsilon \searrow 0}{\longrightarrow} w(s) .
$$

This yields the claim.

Now, let $\omega \geq 0$, and set

$$
w:=\exp (\omega p|\mathbf{s}|+p \sqrt{|\mathbf{s}|})
$$

Then the shift $\left(\tau_{s}\right)_{s \in \mathbb{R}}$ has group type $\theta(\tau)=\omega$ on $\mathrm{L}^{p}(\mathbb{R}, w(s) \mathrm{d} s)$, and

$$
v_{\tau}:=\mathrm{e}^{-\theta(\tau)|\mathbf{s}|}\left\|\tau_{\mathbf{s}}\right\|=\mathrm{e}^{\sqrt{|\mathbf{s}|}}
$$

As $v_{\tau}$ still grows faster than any polynomial, there is no admissible weight $v: \mathbb{R} \rightarrow[1, \infty)$ with $\frac{v_{\tau}}{v} \in \mathrm{L}^{2}(\mathbb{R})$.

The next proposition yields a characterization of group generators with a bounded Sobolev calculus. This is a generalization of [35, Lemma 3.7] which treats the special case where $\omega=0$ and $v$ is a polynomial weight.

Proposition 4.1.6. Let $-\mathrm{i} A$ be the generator of a $C_{0}$-group $\left(U_{s}\right)_{s \in \mathbb{R}}$ on a Banach space $X$, and let $\omega \geq \theta(U)$. Further let $v: \mathbb{R} \rightarrow[1, \infty)$ be an admissible weight. Then the following statements are equivalent:

(i) The mapping $\frac{\mathrm{e}^{-\omega|\mathbf{s}|}}{v} U_{\mathbf{s}}$ has weakly square integrable orbits, that is, for each $x \in X$ and $x^{\prime} \in X^{\prime}$ one has

$$
\frac{\mathrm{e}^{-\omega|\mathbf{s}|}}{v}\left\langle U_{\mathbf{s}} x, x^{\prime}\right\rangle \in \mathrm{L}^{2}(\mathbb{R}) ;
$$


(ii) A has a bounded $\mathrm{W}_{v}^{2}\left(\overline{\mathrm{St}}_{\omega}\right)$-calculus.

In this case,

$$
\left\langle\Phi(f) x, x^{\prime}\right\rangle=\int_{\mathbb{R}} f^{\vee}(s)\left\langle U_{s} x, x^{\prime}\right\rangle \mathrm{d} s
$$

for all $f \in \mathrm{W}_{v}^{2}\left(\overline{\mathrm{St}}_{\omega}\right), x \in X$ and $x^{\prime} \in X^{\prime}$, where $\Phi$ denotes the Sobolev calculus of $A$.

Proof of Proposition 4.1.6. First suppose that (i) holds. By the closed graph theorem there is a constant $c \geq 0$ such that

$$
\left\|\frac{\mathrm{e}^{-\omega|\mathbf{s}|}}{v}\left\langle U_{\mathbf{s}} x, x^{\prime}\right\rangle\right\|_{2} \leq c\|x\|\left\|x^{\prime}\right\|
$$

for all $x \in X$ and $x^{\prime} \in X^{\prime}$. Let $f \in \mathcal{E}\left[\mathrm{St}_{\omega}\right], x \in X$, and $x^{\prime} \in X^{\prime}$. Then, by Proposition 2.5 .14 ,

$$
\begin{aligned}
\left|\left\langle f(A) x, x^{\prime}\right\rangle\right| & =\left|\int_{\mathbb{R}} f^{\vee}(s)\left\langle U_{s} x, x^{\prime}\right\rangle \mathrm{d} s\right| \\
& =\left|\int_{\mathbb{R}}\left(v(s) \mathrm{e}^{\omega|s|} f^{\vee}(s)\right) \cdot\left(\frac{\mathrm{e}^{-\omega|s|}}{v(s)}\left\langle U_{s} x, x^{\prime}\right\rangle\right) \mathrm{d} s\right| \\
& \leq c\|f\|_{\mathrm{W}_{v}^{2}\left(\overline{\mathrm{St}}_{\omega}\right)}\|x\|\left\|x^{\prime}\right\|,
\end{aligned}
$$

where we have used 4.1.2 and the Cauchy-Schwarz inequality for the last estimate. This yields (ii). Now suppose that (ii) holds. By Lemma 3.1.9, we may suppose that $v \in \mathrm{C}^{2}(\mathbb{R})$. Let $\eta \in \mathrm{C}_{\mathrm{c}}^{2}(\mathbb{R})$ with $0 \notin \operatorname{supp}(\eta)$. As $\frac{\mathrm{e}^{-\omega|\mathbf{s}|}}{v} \eta \in \mathrm{C}_{\mathrm{c}}^{2}(\mathbb{R})$, one has

$$
\mathcal{F}\left(\frac{\mathrm{e}^{-\omega|\mathbf{s}|}}{v} \eta\right) \in \bigcap_{\theta>0} \mathcal{E}\left(\mathrm{St}_{\theta}\right) .
$$

Therefore, Proposition 2.5 .14 yields for every $x \in X$ and $x^{\prime} \in X^{\prime}$ that

$$
\begin{aligned}
\left|\int_{\mathbb{R}} \eta(s) \frac{\mathrm{e}^{-\omega|s|}}{v(s)}\left\langle U_{s} x, x^{\prime}\right\rangle \mathrm{d} s\right| & =\left|\int_{\mathbb{R}} \mathcal{F}^{-1} \mathcal{F}\left(\frac{\mathrm{e}^{-\omega \mid \mathbf{s}} \mid}{v} \eta\right)\left\langle U_{s} x, x^{\prime}\right\rangle \mathrm{d} s\right| \\
& =\left|\left\langle\mathcal{F}\left(\frac{\mathrm{e}^{-\omega|\mathbf{s}|}}{v} \eta\right)(A) x, x^{\prime}\right\rangle\right| \\
& \lesssim\left\|\mathcal{F}\left(\frac{\mathrm{e}^{-\omega|\mathbf{s}|}}{v} \eta\right)\right\|_{\mathrm{W}_{v}^{2}\left(\overline{\left.\mathrm{St}_{\omega}\right)}\right.}\|x\|\left\|x^{\prime}\right\| \\
& =\|\eta\|_{2}\|x\|\left\|x^{\prime}\right\| .
\end{aligned}
$$

As the space $D=\left\{\eta \in \mathrm{C}_{\mathrm{c}}^{2}(\mathbb{R}) \mid 0 \notin \operatorname{supp}(\eta)\right\}$ is dense in $\mathrm{L}^{2}(\mathbb{R})$, (i) follows. For verify the identity (4.1.2), first note that 4.1.2) holds for all functions $f \in \mathcal{E}\left[\mathrm{St}_{\omega}\right]$ by Lemma 4.1.2 and Proposition 2.5.14. Now, let $f \in \mathrm{W}_{v}^{2}\left(\overline{\mathrm{St}}_{\omega}\right)$ and choose a sequence $\left(\psi_{n}\right)_{n}$ in $\mathrm{H}_{0}^{\infty}\left[\mathrm{St}_{\omega}\right]$ 
with $\psi_{n} \rightarrow f$ in $\mathrm{W}_{v}^{2}\left(\overline{\mathrm{St}}_{\omega}\right)$. Then, $v \mathrm{e}^{\omega \mid \mathbf{s}}\left|\psi_{n}^{\vee} \rightarrow v \mathrm{e}^{\omega \mid \mathbf{s}}\right| f^{\vee}$ in $\mathrm{L}^{2}(\mathbb{R})$. Hence, by (i), whenever $x \in X$ and $x^{\prime} \in X^{\prime}$,

$$
\begin{aligned}
\left\langle\Phi(f) x, x^{\prime}\right\rangle & =\lim _{n \rightarrow \infty}\left\langle\Phi\left(\psi_{n}\right) x, x^{\prime}\right\rangle \\
& =\lim _{n \rightarrow \infty} \int_{\mathbb{R}} \psi_{n}^{\vee}(s)\left\langle U_{s} x, x^{\prime}\right\rangle \mathrm{d} s \\
& =\lim _{n \rightarrow \infty} \int_{\mathbb{R}} v(s) \mathrm{e}^{\omega|s|} \psi_{n}^{\vee}(s) \cdot \frac{\mathrm{e}^{-\omega|s|}}{v(s)}\left\langle U_{s} x, x^{\prime}\right\rangle \mathrm{d} s \\
& =\int_{\mathbb{R}} v(s) \mathrm{e}^{\omega|s|} f^{\vee}(s) \cdot \frac{\mathrm{e}^{-\omega|s|}}{v(s)}\left\langle U_{s} x, x^{\prime}\right\rangle \mathrm{d} s=\int_{\mathbb{R}} f^{\vee}(s)\left\langle U_{s} x, x^{\prime}\right\rangle \mathrm{d} s .
\end{aligned}
$$

\section{The Extended Calculus}

From a bounded Sobolev calculus one obtains an extended calculus by means of the algebraic extension procedure.

Definition 4.1.7. Let $A$ be a strip type operator on a Banach space $X$, let $\omega \geq \omega_{\text {st }}(A)$, let $v: \mathbb{R} \rightarrow[1, \infty)$ be an admissible weight, and suppose that $A$ has a bounded $\mathrm{W}_{v}^{2}\left(\overline{\operatorname{St}}_{\omega}\right)$ calculus. Write $\mathrm{C}_{\omega}:=\mathrm{C}\left(\overline{\mathrm{St}}_{\omega}\right)$ for the space of all continuous functions on $\overline{\mathrm{St}}_{\omega}$, and let $\Phi: \mathrm{W}_{v}^{2}\left(\overline{\mathrm{St}}_{\omega}\right) \rightarrow \mathcal{L}(X)$ denote the Sobolev calculus of $A$. Then the algebraic extension

$$
\Phi:\left[\mathrm{C}_{\omega}\right]_{\mathrm{W}_{v}^{2}\left(\overline{\mathrm{St}}_{\omega}\right)} \rightarrow \mathcal{C}(X)
$$

(see Definition 2.2.3) is called the extended $\mathrm{W}_{v}^{2}\left(\overline{\mathrm{St}}_{\omega}\right)$-calculus or just extended Sobolev calculus of $A$.

Remark 4.1.8. One may argue that only the case $\omega=\omega_{\mathrm{st}}(A)$ is of real interest in Definition 4.1.7. Indeed, our actual goal is to find a calculus which covers all expressions $f(A)$ for $f \in \operatorname{Hör}_{v}^{2}\left(\overline{\mathrm{St}}_{\omega}\right)$. On one hand, for every $\omega>0$ one has $\operatorname{Hör}_{v}^{2}\left(\overline{\operatorname{St}}_{\omega}\right) \subseteq \mathrm{H}^{\infty}\left(\mathrm{St}_{\omega}\right)$ by Lemma 3.3.3. On the other hand, if $\omega>\omega_{\text {st }}(A)$, then an $\mathrm{H}^{\infty}\left(\mathrm{St}_{\omega}\right)$-calculus is already available for $A$. Hence, there is a $\operatorname{Hör}_{v}^{2}\left(\overline{\mathrm{St}}_{\omega}\right)$-calculus as well. However, whether $\omega=$ $\omega_{\mathrm{st}}(A)$ or not makes no difference for the abstract theory presented in the remainder of this section. This is why we stick to the general assumption that $\omega \geq \omega_{\mathrm{st}}(A)$.

The extended Sobolev calculus extends the holomorphic calculus of $A$. This is a corollary of Lemma 4.1.2.

Corollary 4.1.9. In the situation of Definition 4.1.7, let $\theta^{\prime}>\omega$, and let $f: \mathrm{St}_{\theta^{\prime}} \rightarrow \mathbb{C}$ be a holomorphic function such that $f(A)$ is defined in the holomorphic calculus of $A$. Then $\Phi(f)$ is defined in the extended Sobolev calculus of $A$ and

$$
\Phi(f)=f(A) .
$$

Proof. Let

$$
[f]_{\mathrm{Hol}}:=[f]_{\mathcal{E}\left[\mathrm{St}_{\omega}\right]}=\left\{e \in \mathcal{E}\left[\mathrm{St}_{\omega}\right] \mid e f \in \mathcal{E}\left[\mathrm{St}_{\omega}\right]\right\}
$$


For every $e \in[f]_{\text {Hol }}$ one has

$$
(e f)(A)=\Phi(e f)=\Phi(e) \Phi(f)=e(A) \Phi(f)
$$

by Lemma 4.1.2. Consequently, for every $x, y \in X$,

$$
f(A) x=y
$$

if and only if

$$
e(A) y=(e f)(A) x=e(A) \Phi(f) x
$$

for all $e \in[f]_{\mathrm{Hol}}$. As $[f]_{\mathrm{Hol}}$ is an anchor set by hypothesis, it follows that $f(A) x=y$ if and only if $\Phi(f) x=y$. Hence, $f(A)=\Phi(f)$.

From now on we write $f(A)$ instead of $\Phi(f)$ whenever $\Phi(f)$ is defined within the extended Sobolev calculus of $A$.

Proposition 4.1.10. Let $A$ be a strip type operator on some Banach space, let $\omega \geq$ $\omega_{\mathrm{st}}(A)$, and let $v: \mathbb{R} \rightarrow[1, \infty)$ be an admissible weight. Suppose that $A$ has a bounded $\mathrm{W}_{v}^{2}\left(\overline{\mathrm{St}}_{\omega}\right)$-calculus. Then for every function $f \in \operatorname{Hör}_{v}^{2}\left(\overline{\mathrm{St}}_{\omega}\right)$ the operator $f(A)$ is defined in the extended $\mathrm{W}_{v}^{2}\left(\overline{\mathrm{St}}_{\omega}\right)$-calculus and one has

$$
f(A)=\psi(A)^{-1}(\psi f)(A) .
$$

Proof. Let $f \in \operatorname{Hör}_{v}^{2}\left(\overline{\mathrm{St}}_{\omega}\right)$. Then $\psi f \in \mathrm{W}_{v}^{2}\left(\overline{\mathrm{St}}_{\omega}\right)$ by Lemma 3.3.7. As $\psi(A)$ is injective by Corollary 2.3.14, the claim follows.

Remark 4.1.11. Note that in 4.1.3 the function $\psi=\mathrm{e}^{-\mathbf{z}^{2}}$ can be replaced with any other function $\psi \in \mathrm{H}_{0}^{\infty}\left[\mathrm{St}_{\omega}\right]$ such that $\psi(A)$ is injective. Furthermore, recall that by Lemma 2.3.13 the set

$$
\mathcal{E}_{\psi}:=\left\{\tau_{t} \psi \mid t \in \mathbb{R}\right\}
$$

is an anchor set with respect to the holomorphic calculus of $A$ for each non-zero $\psi \in$ $\mathrm{H}_{0}^{\infty}\left(\mathrm{St}_{\theta}\right)$. Hence, $\mathcal{E}_{\psi}$ is also an anchor set with respect to the $\mathrm{W}_{v}^{2}\left(\overline{\operatorname{St}}_{\omega}\right)$-calculus of $A$. Therefore, every function $f \in \operatorname{Hör}_{v}^{2}\left(\overline{\mathrm{St}}_{\omega}\right)$ is even anchored by the set $\mathcal{E}_{\psi}$ regardless of whether $\psi(A)$ is an injective operator or not.

Definition 4.1.12. Let $A, \omega$, and $v$ be given as in Definition 4.1.7. Then the mapping

$$
\operatorname{Hör}_{v}^{2}\left(\overline{\mathrm{St}}_{\omega}\right) \rightarrow \mathcal{C}(X), \quad f \mapsto f(A),
$$

is called the $\operatorname{Hör}_{v}^{2}\left(\overline{\mathrm{St}}_{\omega}\right)$-calculus or simply the Hörmander calculus of $A$. We say that $A$ has a bounded $\operatorname{Hör}_{v}^{2}\left(\overline{\mathrm{St}}_{\omega}\right)$-calculus if $f(A) \in \mathcal{L}(X)$ for all $f \in \operatorname{Hör}_{v}^{2}\left(\overline{\mathrm{St}}_{\omega}\right)$.

Remarks 4.1.13.

1) Let $A$ be an operator with bounded $\operatorname{Hör}_{v}^{2}\left(\overline{\mathrm{St}}_{\omega}\right)$-calculus. Then one can show similarly to Remark 2.3 .12 that there is a constant $c \geq 0$ such that

$$
\|f(A)\| \leq c\|f\|_{\operatorname{Hör}_{v}^{2}\left(\overline{\operatorname{St}}_{\omega}\right)} \quad\left(f \in \operatorname{Hör}_{v}^{2}\left(\overline{\mathrm{St}}_{\omega}\right)\right) .
$$

2) It would be more accurate to speak of a "calculus of Hörmander-Mikhlin type" in Definition 4.1.12, since Hörmander himself did not introduce this calculus. However, since the term "Hörmander functional calculus" is already established in the works of Kriegler and Weis (see [32, 34], and [36]), we will keep the given terminology. 


\section{Convergence Lemma}

We prove a convergence lemma for the Hörmander calculus. To this end, recall that a set $\mathcal{E} \subseteq \mathrm{W}_{v}^{2}\left(\overline{\mathrm{St}}_{\omega}\right)$ is said to have total range with respect to the Sobolev calculus if the set

$$
\{\psi(A) x \mid \psi \in \mathcal{E}, x \in X\}=\bigcup_{\psi \in \mathcal{E}} \operatorname{ran} \psi(A)
$$

is total in $X$. Moreover, if $A$ is densely defined, the canonical examples for sets $\mathcal{E}$ with total range are given by

$$
\mathcal{E}=\mathcal{E}_{\psi}=\left\{\tau_{t} \psi \mid t \in \mathbb{R}\right\},
$$

where $0 \neq \psi \in \mathrm{H}_{0}^{\infty}\left[\mathrm{St}_{\omega}\right]$ (see Lemma 2.3.13).

Theorem 4.1.14 (Convergence lemma). Let $A$ be a strip type operator on a Banach space $X$, let $\omega \geq \omega_{\mathrm{st}}(A)$, let $v: \mathbb{R} \rightarrow[1, \infty)$ be an admissible weight, and suppose that $A$ has a bounded $\mathrm{W}_{v}^{2}\left(\overline{\mathrm{St}}_{\omega}\right)$-calculus. Let $f \in \operatorname{Hör}_{v}^{2}\left(\overline{\mathrm{St}}_{\omega}\right)$, and let $\left(f_{n}\right)_{n}$ be a sequence in $\mathrm{Hör}_{v}^{2}\left(\overline{\mathrm{St}}_{\omega}\right)$ satisfying the following conditions:

1) There is a set $\mathcal{E} \subseteq \mathrm{H}_{0}^{\infty}\left[\mathrm{St}_{\omega}\right]$ with total range (with respect to the $\mathrm{W}_{v}^{2}\left(\overline{\mathrm{St}}_{\omega}\right)$-calculus of $A$ ) such that

$$
\left\|\psi\left(f_{n}-f\right)\right\|_{\mathrm{W}_{v}^{2}\left(\overline{\mathrm{St}}_{\omega}\right)} \stackrel{n \rightarrow \infty}{\longrightarrow} 0 \quad(\psi \in \mathcal{E}) ;
$$

2) $\sup _{n \in \mathbb{N}}\left\|f_{n}(A)\right\|<\infty$.

Then, $f(A) \in \mathcal{L}(X)$ and $f_{n}(A) \rightarrow f(A)$ strongly on $X$.

Proof. Observe that

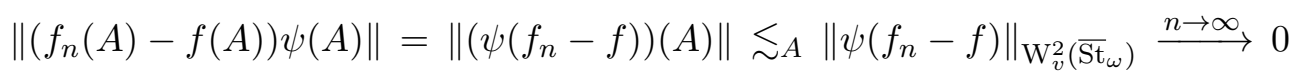

for all $\psi \in \mathcal{E}$. As $\mathcal{E}$ has total range and the sequence $\left(f_{n}(A)\right)_{n}$ is uniformly bounded, the claim follows.

\section{Remarks 4.1.15.}

1) Theorem 4.1.14 extends the holomorphic convergence lemma (Theorem 2.3.10 in the following sense: Let $\theta>\omega$, and let $\left(f_{n}\right)_{n}$ be a uniformly bounded sequence in $\mathrm{H}^{\infty}\left(\mathrm{St}_{\theta}\right)$ that converges pointwise on $\mathrm{St}_{\theta}$ to a function $f \in \mathrm{H}^{\infty}\left(\mathrm{St}_{\theta}\right)$. Then,

$$
\left\|\psi\left(f_{n}-f\right)\right\|_{\mathrm{W}_{v}^{2}\left(\overline{\mathrm{St}}_{\omega}\right)} \stackrel{n \rightarrow \infty}{\longrightarrow} 0 \quad\left(\psi \in \mathcal{E}\left(\mathrm{St}_{\theta}\right)\right) .
$$

Indeed, for each $\psi \in \mathcal{E}\left(\mathrm{St}_{\theta}\right)$ and $\theta_{0} \in(\omega, \theta)$ one has

$$
\left\|\psi\left(f_{n}-f\right)\right\|_{\mathrm{W}_{v}^{2}\left(\overline{\mathrm{St}}_{\omega}\right)} \lesssim \sum_{r= \pm \theta_{0}}\left\|\psi_{r}\left(\left(f_{n}\right)_{r}-f_{r}\right)\right\|_{2} \stackrel{n \rightarrow \infty}{\longrightarrow} 0 .
$$

As the set $\mathcal{E}\left(\mathrm{St}_{\theta}\right)$ has total range if $A$ is densely defined (see Lemma 2.3.13), the sequence $\left(f_{n}\right)_{n}$ and $f$ therefore satisfy condition 1$)$ of Theorem 4.1.14. 
2) In fact, Theorem 4.1.14 rather is a property of the extended Sobolev calculus than the Hörmander calculus. One can drop the conditions $f_{n}, f \in \operatorname{Hör}_{v}^{2}\left(\overline{\mathrm{St}}_{\omega}\right)$ in the hypothesis and simply require $f_{n}(A)$ and $f(A)$ to be defined in the extended Sobolev calculus instead. (Of course, one also needs to guarantee that $\psi f, \psi f_{n} \in \mathrm{W}_{v}^{2}\left(\overline{\mathrm{St}}_{\omega}\right)$ for all $\psi \in \mathcal{E}$.) Then the statement still holds (with the same proof). However, the convergence lemma is only applied to the Hörmander calculus in the sequel which is why we stick with the given formulation.

3) One can also formulate a "weak" convergence lemma for the Hörmander calculus which infers a weak convergence under less restrictive assumptions (cf. Theorem 4.2.17 and Remark 4.2.18).

In the next lemma, we show that for a given function $f \in \operatorname{Hör}_{v}^{2}\left(\overline{\mathrm{St}}_{\omega}\right)$ one can always find a bounded sequence $\left(f_{n}\right)_{n}$ in $\mathrm{H}_{0}^{\infty}\left[\mathrm{St}_{\omega}\right]$ such that condition 1) of Theorem 4.1.14 is satisfied. Moreover, this sequence may be chosen in $\mathrm{H}_{0}^{\infty}\left(\mathrm{St}_{\theta}\right)$ for any width $\theta>\omega$.

Lemma 4.1.16. Let $\theta>\omega \geq 0$, and let $v: \mathbb{R} \rightarrow[1, \infty)$ be an admissible weight. Then there is a constant $K \geq 0$ with the following property: For each $f \in \operatorname{Hör}_{v}^{2}\left(\overline{\operatorname{St}}_{\omega}\right)$ there is a sequence $\left(\varphi_{n}\right)_{n}$ in $\mathrm{H}_{0}^{\infty}\left(\mathrm{St}_{\theta}\right)$ such that

$$
\sup _{n \in \mathbb{N}}\left\|\varphi_{n}\right\|_{\operatorname{Hör}_{v}^{2}\left(\overline{\operatorname{St}}_{\omega}\right)} \leq K\|f\|_{\operatorname{Hör}_{v}^{2}\left(\overline{\operatorname{St}}_{\omega}\right)}
$$

and

$$
\left\|\tau_{t} \psi \cdot\left(\varphi_{n}-f\right)\right\|_{\mathrm{W}_{v}^{2}\left(\overline{\mathrm{St}}_{\omega}\right)} \stackrel{n \rightarrow \infty}{\longrightarrow} 0
$$

for all $t \in \mathbb{R}$.

Proof. First we construct a sequence $\left(\phi_{n}\right)_{n}$ in $\mathrm{H}_{0}^{\infty}\left(\mathrm{St}_{\theta}\right)$ such that

$$
\left\|\psi\left(\phi_{n}-\mathbf{1}\right)\right\|_{\mathrm{W}_{v}^{2}\left(\overline{\mathrm{St}}_{\omega}\right)} \stackrel{n \rightarrow \infty}{\longrightarrow} 0 \quad\left(\psi \in \mathrm{H}_{0}^{\infty}\left(\mathrm{St}_{\theta}\right)\right)
$$

and

$$
\sup _{n \in \mathbb{N}}\left\|\phi_{n}\right\|_{\operatorname{Hör}_{v}^{2}\left(\overline{\operatorname{St}_{\omega}}\right)}<\infty .
$$

To this end, let $\phi \in \mathrm{H}_{0}^{\infty}\left(\mathrm{St}_{\theta}\right)$ with $\phi(0)=1$, and set

$$
\phi_{n}:=\phi\left(\frac{\mathbf{z}}{n}\right) \quad \text { and } \quad \phi_{n, r}:=\left(\phi_{n}\right)_{r} \quad(r \in\{-\omega, \omega\}) .
$$

Further let $N \in \mathbb{N}$ with $v \lesssim(1+|\mathbf{s}|)^{N}$ (see Lemma 3.1.7). For every $\psi \in \mathrm{H}_{0}^{\infty}\left(\mathrm{St}_{\theta}\right)$ one has

$$
\begin{array}{r}
\left\|\psi\left(\phi_{n}-\mathbf{1}\right)\right\|_{\mathrm{W}_{v}^{2}\left(\overline{\mathrm{St}_{\omega}}\right)} \lesssim v \sum_{r= \pm \omega}\left\|\psi_{r}\left(\phi_{n, r}-\mathbf{1}\right)\right\|_{\mathrm{W}^{N, 2}(\mathbb{R})} \\
\lesssim \sum_{r= \pm \omega} \sum_{k=0}^{N} \frac{1}{n^{k}}\left\|\left(\psi_{r}\right)^{(N-k)}\left(\phi_{n, r}-\mathbf{1}\right)^{(k)}\right\|_{2}
\end{array}
$$




$$
\leq \sum_{\substack{r= \pm \omega \\ n \rightarrow \infty}} 0
$$

by the dominated convergence theorem. Similarly one estimates

$$
\begin{aligned}
\left\|\phi_{n}\right\|_{\mathrm{Hör}_{v}^{2}(\mathbb{R})} & \lesssim v \sup _{t \in \mathbb{R}} \sum_{r= \pm \omega}\left\|\tau_{r} \psi \cdot \phi_{n, r}\right\|_{\mathrm{W}^{N, 2}(\mathbb{R})} \\
& \lesssim \sup _{t \in \mathbb{R}}\left(\sum_{r= \pm \omega} \sum_{k=0}^{N} \frac{1}{n^{k}}\left\|\left(\tau_{r} \psi\right)^{(N-k)}\right\|_{2}\left\|\phi^{(k)}\right\|_{\infty, \overline{\mathrm{St}} \omega}\right) \\
& \leq \sum_{k=0}^{N}\left\|\psi^{(N-k)}\right\|_{2}\left\|\phi^{(k)}\right\|_{\infty, \overline{\mathrm{St}} \omega},
\end{aligned}
$$

whence $\sup _{n \in \mathbb{N}}\left\|\phi_{n}\right\|_{\mathrm{Hör}_{v}^{2}\left(\overline{\mathrm{St}}_{\omega}\right)}<\infty$. Therefore, the sequence $\left(\phi_{n}\right)_{n}$ has the claimed properties. Now, fix a function $f \in \operatorname{Hör}_{v}^{2}\left(\overline{\operatorname{St}}_{\omega}\right)$ and set $g_{n}:=\phi_{n} f$. Then $\left(g_{n}\right)_{n}$ is a sequence in $\mathrm{W}_{v}^{2}\left(\overline{\mathrm{St}}_{\omega}\right)$,

$$
\sup _{n \in \mathbb{N}}\left\|g_{n}\right\|_{\operatorname{Hör}_{v}^{2}\left(\overline{\operatorname{St}}_{\omega}\right)} \lesssim v \sup _{n \in \mathbb{N}}\left\|\phi_{n}\right\|_{\operatorname{Hör}_{v}^{2}\left(\overline{\mathrm{St}}_{\omega}\right)}\|f\|_{\operatorname{Hör}_{v}^{2}\left(\overline{\operatorname{St}_{\omega}}\right)}<\infty
$$

and

$$
\left\|\psi^{2}\left(g_{n}-f\right)\right\|_{\mathrm{W}_{v}^{2}\left(\overline{\mathrm{St}_{\omega}}\right)} \lesssim v\left\|\psi\left(\phi_{n}-\mathbf{1}\right)\right\|_{\mathrm{W}_{v}^{2}\left(\overline{\mathrm{St}_{\omega}}\right)}\|\psi f\|_{\mathrm{W}_{v}^{2}\left(\overline{\mathrm{St}_{\omega}}\right)} \stackrel{n \rightarrow \infty}{\longrightarrow} 0
$$

for each $\psi \in \mathrm{H}_{0}^{\infty}\left[\mathrm{St}_{\omega}\right]$. In particular, if we set $\psi:=\psi^{\frac{1}{2}}=\mathrm{e}^{-\frac{1}{2} \mathbf{z}^{2}}$, then

$$
\left\|\tau_{t} \psi \cdot\left(g_{n}-f\right)\right\|_{\mathrm{W}_{v}^{2}\left(\overline{\mathrm{St}}_{\omega}\right)}=\left\|\left(\tau_{t} \psi\right)^{2} \cdot\left(g_{n}-f\right)\right\|_{\mathrm{W}_{v}^{2}\left(\overline{\mathrm{St}}_{\omega}\right)} \stackrel{n \rightarrow \infty}{\longrightarrow} 0
$$

for all $t \in \mathbb{R}$. Finally, recall that $\mathrm{H}_{0}^{\infty}\left(\mathrm{St}_{\theta}\right)$ is dense in $\mathrm{W}_{v}^{2}\left(\overline{\mathrm{St}}_{\omega}\right)$, whence one can choose $\varphi_{n} \in \mathrm{H}_{0}^{\infty}\left(\mathrm{St}_{\theta}\right)$ with

$$
\left\|g_{n}-\varphi_{n}\right\|_{\mathrm{W}_{v}^{2}\left(\overline{\mathrm{St}}_{\omega}\right)} \leq \frac{1}{n}\|f\|_{\mathrm{Hör}_{v}^{2}\left(\overline{\mathrm{St}}_{\omega}\right)}
$$

for each $n \in \mathbb{N}$. Then

$$
\begin{aligned}
\left\|\varphi_{n}\right\|_{\mathrm{Hör}_{v}^{2}\left(\overline{\mathrm{St}}_{\omega}\right)} & =\sup _{t \in \mathbb{R}}\left\|\tau_{t} \psi \cdot \varphi_{n}\right\|_{\mathrm{W}_{v}^{2}\left(\overline{\mathrm{St}}_{\omega}\right)} \\
& \leq \sup _{t \in \mathbb{R}}\left(\left\|\tau_{t} \psi \cdot\left(\varphi_{n}-g_{n}\right)\right\|_{\mathrm{W}_{v}^{2}\left(\overline{\mathrm{St}}_{\omega}\right)}+\left\|\tau_{t} \psi \cdot g_{n}\right\|_{\mathrm{W}_{v}^{2}\left(\overline{\mathrm{St}}_{\omega}\right)}\right) \\
& \lesssim v \frac{1}{n}\|\psi\|_{\mathrm{W}_{v}^{2}\left(\overline{\mathrm{St}_{\omega}}\right)}\|f\|_{\mathrm{Hör}_{v}^{2}\left(\overline{\mathrm{St}}_{\omega}\right)}+\left\|g_{n}\right\|_{\mathrm{Hör}_{v}^{2}\left(\overline{\mathrm{St}}_{\omega}\right)} \\
& \lesssim v\left(\|\psi\|_{\mathrm{W}_{v}^{2}\left(\overline{\mathrm{St}_{\omega}}\right)}+\sup _{k \in \mathbb{N}}\left\|\phi_{k}\right\|_{\mathrm{Hör}_{v}^{2}\left(\overline{\mathrm{St}_{\omega}}\right)}\right)\|f\|_{\operatorname{Hör}_{v}^{2}\left(\overline{\mathrm{St}}_{\omega}\right)} .
\end{aligned}
$$

Therefore, we may set

$$
K:=\|\psi\|_{\mathrm{W}_{v}^{2}\left(\overline{\mathrm{St}}_{\omega}\right)}+\sup _{k \in \mathbb{N}}\left\|\phi_{k}\right\|_{\mathrm{Hör}_{v}^{2}\left(\overline{\mathrm{St}}_{\omega}\right)}
$$


(times a constant only depending on $v$ ) to establish (4.1.4). One readily verifies that

$$
\left\|\tau_{t} \psi \cdot\left(\varphi_{n}-f\right)\right\|_{\mathrm{W}_{v}^{2}\left(\overline{\mathrm{St}}_{\omega}\right)} \stackrel{n \rightarrow \infty}{\longrightarrow} 0
$$

for each $t \in \mathbb{R}$. This concludes the proof.

\subsection{A More General Approach To Hörmander Functional Calculi}

In the previous section, the construction of the Hörmander functional calculus was based on the presence of a bounded Sobolev calculus. However, it is possible to define a Hörmander calculus in a less restrictive setting. In this section, we present a way to define a more general (possibly unbounded) Sobolev calculus from which a Hörmander calculus may be constructed. For the case that $A$ is 0 -sectorial or of strip type 0 , this is discussed e.g., in [32], 35], and [36]. Here we give an abstract approach to this problem for a certain subclass of strip type operators. The presented methods generalize those used in [32], 35], and [36].

The contents of this section are not used in Section 4.3 or thereafter. The reader who is more interested in results on bounded Hörmander calculi may skip ahead.

For the first step we introduce unbounded algebra homomorphisms between normed algebras.

Definition 4.2.1. Let $\mathcal{A}, \mathcal{B}$ be normed algebras. A (possibly unbounded) linear operator $\Phi \subseteq \mathcal{A} \oplus \mathcal{B}$ is called a (possibly unbounded) algebra homomorphism if $(x u, y v) \in \Phi$ for all $(x, y),(u, v) \in \Phi$. An algebra homomorphism $\Phi$ is called closable if it is closable as a linear operator, i.e., if the closure $\bar{\Phi}$ in $\mathcal{A} \oplus \mathcal{B}$ is an operator as well.

Remarks 4.2.2. Let $\mathcal{A}, \mathcal{B}$ be normed algebras, and let $\Phi \subseteq \mathcal{A} \oplus \mathcal{B}$ be a possibly unbounded algebra homomorphism.

1) The space $\operatorname{dom} \Phi$ is a subalgebra of $\mathcal{A}$.

2) One has the following:

$$
\forall(x, y),(u, v) \in \bar{\Phi}: \quad(x u, y v) \in \bar{\Phi}
$$

This follows from the continuity of the multiplication on $\mathcal{A}$ and $\mathcal{B}$. As a consequence, if $\Phi$ is closable, then $\bar{\Phi}$ is also an algebra homomorphism.

Definition 4.2.3. Let $A$ be a strip type operator on a Banach space $X$, let $\omega \geq \omega_{\mathrm{st}}(A)$, and let $v: \mathbb{R} \rightarrow[1, \infty)$ be an admissible weight. Define

$$
\Phi_{A, \omega, v, \text { aux }}:=\Phi_{\text {aux }}:=\left\{(\psi, T) \in \mathrm{W}_{v}^{2}\left(\overline{\mathrm{St}}_{\omega}\right) \oplus \mathcal{L}(X) \mid \psi \in \mathcal{E}\left[\mathrm{St}_{\omega}\right], T=\psi(A)\right\} .
$$

Then

$$
\mathcal{E}_{v, A}^{2}\left(\overline{\mathrm{St}}_{\omega}\right):=\mathcal{E}_{v}^{2}\left(\overline{\mathrm{St}}_{\omega}\right):=\operatorname{dom} \bar{\Phi}_{\mathrm{aux}}
$$

is called the algebra of elementary Sobolev functions on the strip $\overline{\mathrm{St}}_{\omega}$ (with respect to $A$ ). If $\Phi_{\text {aux }}$ is closable, then the operator $A$ is said to admit an auxiliary (Sobolev) calculus and $\bar{\Phi}_{\text {aux }}$ is called the auxiliary (Sobolev) calculus of $A$. 
In the following we write $\Phi_{\text {aux }}$ instead of $\Phi_{A, \omega, v \text {,aux }}$ as $A, \omega$, and $v$ are always clear from the context.

Remark 4.2.4. Note that $\Phi_{\text {aux }}$ is just the elementary holomorphic calculus of $A$ but considered as an algebra homomorphism between $\mathrm{W}_{v}^{2}\left(\overline{\mathrm{St}}_{\omega}\right)$ and $\mathcal{L}(X)$.

The auxiliary calculus (if existent) takes over the role of the elementary calculus in this setting.

Definition 4.2.5. Let $A, \omega$, and $v$ be given as in Definition 4.2.3, and suppose that $A$ admits an auxiliary Sobolev functional calculus. Write $\mathrm{C}_{\omega}:=\mathrm{C}\left(\overline{\mathrm{St}}_{\omega}\right)$ and $\mathcal{E}:=\mathcal{E}_{v, A}^{2}\left(\overline{\mathrm{St}}_{\omega}\right)$. Then the algebraic extension

$$
\bar{\Phi}_{\mathrm{aux}}:\left[\mathrm{C}_{\omega}\right]_{\mathcal{E}} \rightarrow \mathcal{C}(X)
$$

is called the extended auxiliary (Sobolev) calculus of $A$.

If $A$ admits an auxiliary calculus, then the extended auxiliary calculus extends the holomorphic calculus of $A$. This is a generalization of Corollary 4.1.9.

Lemma 4.2.6. Let $A, \omega$, and $v$ be given as above, and suppose that $A$ admits an auxiliary calculus. Let $\theta^{\prime}>\omega$, and let $f: \mathrm{St}_{\theta^{\prime}} \rightarrow \mathbb{C}$ be a holomorphic function such that $f(A)$ is defined in the holomorphic calculus of $A$. Then $\bar{\Phi}_{\text {aux }}(f)$ is defined in the extended auxiliary calculus of $A$ and

$$
\bar{\Phi}_{\mathrm{aux}}(f)=f(A) .
$$

Proof. Just observe that $\bar{\Phi}_{\text {aux }}(\psi)=\psi(A)$ for all $\psi \in \mathcal{E}\left[\mathrm{St}_{\omega}\right]$. Arguing as in the proof of Corollary 4.1 .9 one concludes the claim.

From now on, whenever $\bar{\Phi}_{\text {aux }}(f)$ is defined within the extended auxiliary calculus of $A$, we write $f(A):=\bar{\Phi}_{\text {aux }}(f)$.

So far the setting is very general and this comes with a big drawback. Without additional information on $A$ there is not much that can be said about the elementary Sobolev algebra or the auxiliary calculus. In particular, it is not clear whether an extended auxiliary calculus covers the class of Hörmander functions, or when an operator admits an auxiliary calculus to begin with.

The next definition introduces a criterion for the existence of an auxiliary calculus (see Proposition 4.2.9).

Definition 4.2.7. Let $A, \omega$, and $v$ be given as in Definition 4.2.3. Set

$$
\mathcal{W}_{A, \omega, v}:=\mathcal{W}_{A}:=\left\{h \in \mathcal{E}\left[\mathrm{St}_{\omega}\right] \mid \forall \psi \in \mathcal{E}\left[\mathrm{St}_{\omega}\right]:\|(\psi h)(A)\| \lesssim_{h}\|\psi\|_{\mathrm{W}_{v}^{2}\left(\overline{\mathrm{St}}_{\omega}\right)}\right\} .
$$

The operator $A$ is said to be $(\omega, v)$-Sobolev anchored or just Sobolev anchored if $\mathcal{W}_{A}$ is an anchor set with respect to the holomorphic calculus of $A$. 
It is possible to characterize the elements of $\mathcal{W}_{A}$. Note the similarities to Proposition 4.1 .6 .

Proposition 4.2.8. Let $A, \omega$, and $v$ be given as in Definition 4.2.7. Further, let $h \in$ $\mathcal{E}\left[\mathrm{St}_{\omega}\right]$ and set $U_{h}(\mathbf{z}, s):=\mathrm{e}^{-\mathrm{i} s \mathbf{z}} h$ for each $s \in \mathbb{R}$. Then the following statements are equivalent:

(i) $h \in \mathcal{W}_{A, \omega, v}$;

(ii) $\exists c \geq 0, \theta>\omega: \forall \psi \in \mathrm{H}_{0}^{\infty}\left(\mathrm{St}_{\theta}\right): \quad\|(\psi h)(A)\| \leq c\|\psi\|_{\mathrm{W}_{v}^{2}\left(\overline{\mathrm{St}_{\omega}}\right)}$;

(iii) $\forall x \in X, x^{\prime} \in X^{\prime}: \quad \frac{\mathrm{e}^{-\omega|\mathbf{s}|}}{v}\left\langle U_{h}(A, \mathbf{s}) x, x^{\prime}\right\rangle \in \mathrm{L}^{2}(\mathbb{R})$.

Proof. Clearly (i) implies (ii). For the converse implication let

$$
T_{h}: \mathrm{H}_{0}^{\infty}\left(\mathrm{St}_{\theta}\right) \rightarrow \mathcal{L}(X), \quad T_{h} \psi:=(\psi h)(A)=\psi(A) h(A) .
$$

By (ii), $T_{h}$ can be continuously extended to $\mathrm{W}_{v}^{2}\left(\overline{\mathrm{St}}_{\omega}\right)$. By abuse of notation, we denote this extension also by $T_{h}$. Arguing similarly to the proof of Lemma 4.1.2, one can show that $T_{h} f=f(A) h(A)$ for all $f \in \mathcal{E}\left[\mathrm{St}_{\omega}\right]$. This yields (i). To prove the equivalence of (i) and (iii), first note that

$$
f(A) h(A)=\int_{\mathbb{R}} f^{\vee}(s) U_{h}(A, s) \mathrm{d} s
$$

for all $f \in \mathcal{E}\left[\mathrm{St}_{\omega}\right]$, where integral on the right converges absolutely in the operator norm. Indeed, writing the operators $U_{h}(A, s)$ as Cauchy integrals, one easily verifies that the mapping

$$
\mathbb{R} \rightarrow \mathcal{L}(X), \quad s \mapsto U_{h}(A, s),
$$

is continuous and that one has $\left\|U_{h}(A, s)\right\| \lesssim_{h, \delta} \mathrm{e}^{\delta|s|}$ for all $\delta>\omega$. Hence, whenever $\delta>\omega$ and $f \in \mathcal{E}\left[\mathrm{St}_{\delta}\right] \subseteq \mathrm{W}_{v}^{2}\left(\overline{\mathrm{St}}_{\delta}\right)$, the right hand side in 4.2 .1 well defined and absolutely convergent, since $\mathrm{e}^{\delta|\mathbf{s}|} f^{\vee} \in \mathrm{L}^{1}(\mathbb{R})$ in this case. Consequently, the integral in (4.2.1) is also well defined for every choice of $f \in \mathcal{E}\left[\mathrm{St}_{\omega}\right]$. Moreover, for every $f \in \mathcal{E}\left[\mathrm{St}_{\omega}\right]$ and suitable $\delta>0$ one has

$$
\begin{aligned}
f(A) h(A) & =\frac{1}{2 \pi \mathrm{i}} \int_{\partial \mathrm{St}_{\delta}} f(z) h(z) R(z, A) \mathrm{d} z \\
& =\frac{1}{2 \pi \mathrm{i}} \int_{\partial \mathrm{St}_{\delta}}\left(\int_{\mathbb{R}} f^{\vee}(s) \mathrm{e}^{-\mathrm{i} s z} \mathrm{~d} s\right) h(z) R(z, A) \mathrm{d} z \\
& =\frac{1}{2 \pi \mathrm{i}} \int_{\partial \mathrm{St}_{\delta}} \int_{\mathbb{R}} f^{\vee}(s) \mathrm{e}^{-\mathrm{i} s z} h(z) R(z, A) \mathrm{d} s \mathrm{~d} z \\
& =\frac{1}{2 \pi \mathrm{i}} \int_{\mathbb{R}} \int_{\partial \mathrm{St}_{\delta}} f^{\vee}(s) \mathrm{e}^{-\mathrm{i} s z} h(z) R(z, A) \mathrm{d} z \mathrm{~d} s \\
& =\int_{\mathbb{R}} f^{\vee}(s)\left(\frac{1}{2 \pi \mathrm{i}} \int_{\partial \mathrm{St}_{\delta}} \mathrm{e}^{-\mathrm{i} s z} h(z) R(z, A) \mathrm{d} z\right) \mathrm{d} s \\
& =\int_{\mathbb{R}} f^{\vee}(s) U_{h}(A, s) \mathrm{d} s,
\end{aligned}
$$


which is 4.2.1. Now one can adapt the proof of Proposition 4.1.6 to arrive at the claim.

Next, we show that $A$ admits an auxiliary calculus if $A$ is Sobolev anchored. In this case, one can also say more about the elementary Sobolev algebra and even obtains a weak representation formula for the auxiliary calculus.

Proposition 4.2.9. Let $A$ be a strip type operator on a Banach space $X$, let $\omega \geq$ $\omega_{\mathrm{st}}(A)$, and let $v: \mathbb{R} \rightarrow[1, \infty)$ be an admissible weight. Suppose that $A$ is $(\omega, v)$-Sobolev anchored. Then the following statements hold:

a) $\Phi_{\mathrm{aux}}$ is closable, i.e., $A$ admits an auxiliary Sobolev calculus.

b) For each $f \in \mathrm{W}_{v}^{2}\left(\overline{\mathrm{St}}_{\omega}\right)$ and $h \in \mathcal{W}_{A}$ one has $f h \in \mathcal{E}_{v}^{2}\left(\overline{\mathrm{St}}_{\omega}\right)$. Moreover,

$$
\left\langle\bar{\Phi}_{\text {aux }}(f h) x, x^{\prime}\right\rangle=\int_{\mathbb{R}} f^{\vee}(s)\left\langle U_{h}(A, s) x, x^{\prime}\right\rangle \mathrm{d} s
$$

for all $x \in X$ and $x^{\prime} \in X^{\prime}$, where $U_{h}(\mathbf{z}, s):=\mathrm{e}^{-\mathrm{i} s \mathbf{z}} h$ for $s \in \mathbb{R}$.

Proof. For a), let $\left(\psi_{n}, T_{n}\right) \in \Phi_{\text {aux }}$ with $\psi_{n} \rightarrow 0$ in $\mathrm{W}_{v}^{2}\left(\overline{\mathrm{St}}_{\omega}\right)$ and $T_{n}=\psi_{n}(A) \rightarrow T$ in $\mathcal{L}(X)$. We need to show that $T=0$. Note that for every $h \in \mathcal{W}_{A}$ one has

$$
h(A) T=\lim _{n \rightarrow \infty}\left(h \psi_{n}\right)(A)=0
$$

since $\psi_{n} \rightarrow 0$ in $\mathrm{W}_{v}^{2}\left(\overline{\mathrm{St}}_{\omega}\right)$. Hence,

$$
\operatorname{ran} T \subseteq \bigcap_{h \in \mathcal{W}_{A}} \operatorname{ker} h(A)=\{0\}
$$

i.e., $T=0$. For b), fix $f \in \mathrm{W}_{v}^{2}\left(\overline{\mathrm{St}}_{\omega}\right)$ and $h \in \mathcal{W}_{A}$. Choose $\psi_{n} \in \mathcal{E}\left[\overline{\operatorname{St}}_{\omega}\right]$ with $\psi_{n} \rightarrow f$ in $\mathrm{W}_{v}^{2}\left(\overline{\mathrm{St}}_{\omega}\right)$. Then, $\psi_{n} h \rightarrow f h$ in $\mathrm{W}_{v}^{2}\left(\overline{\mathrm{St}}_{\omega}\right)$. Moreover, by the definition of $\mathcal{W}_{A}$, the sequence $\left(\psi_{n}(A) h(A)\right)_{n}$ is a Cauchy sequence and therefore converges to some operator $T \in \mathcal{L}(X)$. Hence, $(f h, T) \in \bar{\Phi}_{\text {aux }}$. Moreover,

$$
\psi_{n}(A) h(A)=\int_{\mathbb{R}} \psi_{n}^{\vee}(s) U_{h}(A, s) \mathrm{d} s
$$

for each $n \in \mathbb{N}$, by (4.2.1). The claim may now be concluded from Proposition 4.2.8.(iii).

Remark 4.2.10. One does not need $A$ to be Sobolev anchored to conclude that $f h \in$ $\mathcal{E}_{v}^{2}\left(\overline{\mathrm{St}}_{\omega}\right)$ whenever $f \in \mathrm{W}_{v}^{2}\left(\overline{\mathrm{St}}_{\omega}\right)$ and $h \in \mathcal{W}_{A}$. However, if $A$ is Sobolev anchored, then it follows that $f(A)$ is defined in the extended auxiliary calculus for every $f \in \mathrm{W}_{v}^{2}\left(\overline{\mathrm{St}}_{\omega}\right)$, since every Sobolev function is anchored by the set $\mathcal{W}_{A}$.

We give an example for an operator which does not generate a $C_{0}$-group but still admits an auxiliary Sobolev calculus. 
Example 4.2.11. Consider the (negative) Laplacian $-\Delta$ on $L^{p}(\mathbb{R})$ for $1<p<\infty$, $p \neq 2$. Then $-\Delta$ is a strip type operator of strip type 0 (see [23, p. 236]). However, the operators $\mathrm{e}^{\text {is } \Delta}$ are unbounded for all $s \neq 0$ (see [23, Proposition 8.3.8]). Hence, $-\Delta$ does not generate a $C_{0}$-group on $\mathrm{L}^{p}(\mathbb{R})$. On the other hand, by [23, Theorem 6.2.b)] (which is a reformulation of the Mikhlin multiplier theorem),

$$
\begin{aligned}
& \left\|\mathrm{e}^{\mathrm{i} t \Delta} R(\lambda,-\Delta)\right\| \lesssim \max _{k=0,1}\left\|\mathrm{~s}^{k}\left(\frac{\mathrm{e}^{\mathrm{i} t \mathbf{s}^{2}}}{\lambda+\mathbf{s}^{2}}\right)^{(k)}\right\|_{\infty, \mathbb{R} \backslash\{0\}} \\
& \leq\left\|\frac{\mathrm{e}^{\mathrm{i} t \mathbf{s}^{2}}}{\lambda+\mathbf{s}^{2}}\right\|_{\infty, \mathbb{R} \backslash\{0\}}+\left\|\mathrm{i} t \cdot \frac{2 \mathbf{s}^{2} \mathrm{e}^{\mathrm{i} t \mathbf{s}^{2}}}{\lambda+\mathbf{s}^{2}}-\frac{2 \mathbf{s}^{2} \mathrm{e}^{\mathrm{i} t \mathbf{s}^{2}}}{\left(\lambda+\mathbf{s}^{2}\right)^{2}}\right\|_{\infty, \mathbb{R} \backslash\{0\}} \lesssim 1+|t|
\end{aligned}
$$

for all $t \in \mathbb{R}$ and $|\operatorname{Im} \lambda|>0$. Hence, by Proposition 4.2.8. $(\lambda-\mathbf{z})^{-1} \in \mathcal{W}_{-\Delta, 0, v}$ whenever $v: \mathbb{R} \rightarrow[1, \infty)$ is an admissible weight with $\frac{1+|\mathbf{s}|}{v} \in \mathrm{L}^{2}(\mathbb{R})$. As $R(\lambda,-\Delta)$ is injective, it follows that $-\Delta$ is $(0, v)$-Sobolev anchored for each such $v$. The operator $-\Delta$ is even densely Sobolev anchored (see Definition 4.2.15), since each resolvent $R(\lambda,-\Delta)$ has dense range.

Remark 4.2.12. The question whether $-\Delta$ also admits a bounded Sobolev calculus on $\mathrm{L}^{p}(\mathbb{R})$ remains open for now. However, the author conjectures that this is not the case.

For Sobolev anchored operators we recover a Hörmander functional calculus.

Proposition 4.2.13. Let $A$ be a strip type operator on a Banach space $X$, let $\omega \geq$ $\omega_{\mathrm{st}}(A)$, and let $v: \mathbb{R} \rightarrow[1, \infty)$ be an admissible weight. Suppose that $A$ is $(\omega, v)$-Sobolev anchored. Then for each $f \in \operatorname{Hör}_{v}^{2}\left(\overline{\mathrm{St}}_{\omega}\right)$ the operator $f(A)$ is defined within the extended auxiliary calculus of $A$.

Proof. Let $f \in \operatorname{Hör}_{v}^{2}\left(\mathrm{St}_{\omega}\right)$. Then, $\psi f \in \mathrm{W}_{v}^{2}\left(\overline{\mathrm{St}}_{\omega}\right)$. Hence, by Proposition 4.2.9. b), $h \psi f \in \mathcal{E}_{v}^{2}\left(\overline{\mathrm{St}}_{\omega}\right)$ for each $h \in \mathcal{W}_{A}$. As $\mathcal{W}_{A}$ is an anchor set and $\psi(A)$ is injective, $\psi \cdot \mathcal{W}_{A}$ is an anchor set as well. Hence, $f$ is anchored in $\mathcal{E}_{v}^{2}\left(\overline{\mathrm{St}}_{\omega}\right)$, i.e., $f(A)$ is defined within the extended auxiliary calculus of $A$.

Remark 4.2.14 (Connection to Kriegler and Weis's work). The following setting is considered in [36]: Let $v=(1+|\mathbf{s}|)^{\beta}$ for some $\beta>\frac{1}{2}$, and let $A$ be an injective 0 -sectorial operator on a Banach space $X$ such that $\operatorname{dom} A \cap \operatorname{ran} A$ is dense in $X$. For $\theta \in \mathbb{R}$ define

$$
\rho_{\theta}(z):=z^{\theta}(1+z)^{-2 \theta} \quad\left(z \in \mathrm{S}_{\frac{\pi}{2}}\right) .
$$

The standing assumption on $A$ in [36] is that there are constants $\theta, c>0$ such that

$$
\int_{\mathbb{R}}\left|\frac{1}{v(s)}\left\langle A^{-\mathrm{i} s} y, x^{\prime}\right\rangle\right|^{2} \mathrm{~d} s \leq c\left\|\rho_{-\theta}(A) y\right\|^{2}\left\|x^{\prime}\right\|^{2}
$$

for all $y \in \operatorname{ran} \rho_{\theta}(A)$ and $x^{\prime} \in X^{\prime}$ (cf. [35, (3.9)]). As $\rho_{-\theta}(A)=\rho_{\theta}(A)^{-1}$, this rewrites to the condition that

$$
\int_{\mathbb{R}}\left|\frac{1}{v(s)}\left\langle A^{-\mathrm{i} s} \rho_{\theta}(A) x, x^{\prime}\right\rangle\right|^{2} \mathrm{~d} s \leq c\|x\|^{2}\left\|x^{\prime}\right\|^{2}
$$


for all $x \in X$ and $x^{\prime} \in X^{\prime}$. Hence, by (a sectorial analogue of) Proposition 4.2.8. condition 4.2 .3 is equivalent to saying that $\rho_{\theta} \in \mathcal{W}_{A}$ (where the definition of $\mathcal{W}_{A}$ is adapted accordingly to the sectorial case). Since $A$ is injective, so is $\rho_{\theta}(A)=A^{\theta}(\mathrm{I}+A)^{-\theta}$. Therefore, if $\rho_{\theta} \in \mathcal{W}_{A}$, then $A$ is $(0, v)$-Sobolev anchored.

\section{Convergence Lemmata for the Auxiliary Calculus}

One can generalize Theorem 4.1.14 to a convergence lemma for the extended auxiliary calculus. For this we introduce the following terminology.

Definition 4.2.15. Let $A, \omega$, and $v$ be given as in Definition 4.2.3. Then $A$ is called densely $(\omega, v)$-Sobolev anchored, if the set $\mathcal{W}_{A}$ has total range with respect to the holomorphic calculus of $A$.

Theorem 4.2.16 (Convergence lemma). Let $A$ be a strip type operator on a Banach space $X$, let $\omega \geq \omega_{\mathrm{st}}(A)$, and let $v: \mathbb{R} \rightarrow[1, \infty)$ be an admissible weight. Suppose that $A$ is densely $(\omega, v)$-Sobolev anchored. Let $f, f_{n} \in \mathrm{C}\left(\overline{\mathrm{St}}_{\omega}\right)$ such that the operators $f(A)$ and $f_{n}(A)$ are defined in the extended auxiliary calculus of $A$. Further suppose that:

1) there is a set $\mathcal{E} \subseteq \mathcal{E}_{v, A}^{2}\left(\overline{\mathrm{St}}_{\omega}\right)$ with total range such that $f \psi, f_{n} \psi \in \mathrm{W}_{v}^{2}\left(\overline{\mathrm{St}}_{\omega}\right)$ and

$$
\left\|\psi\left(f_{n}-f\right)\right\|_{\mathrm{W}_{v}^{2}\left(\overline{\mathrm{St}}_{\omega}\right)} \stackrel{n \rightarrow \infty}{\longrightarrow} 0
$$

for all $\psi \in \mathcal{E}$

2) $\sup _{n \in \mathbb{N}}\left\|f_{n}(A)\right\|<\infty$.

Then, $f(A) \in \mathcal{L}(X)$ and $f_{n}(A) \rightarrow f(A)$ strongly on $X$.

The proof is just a slight variation of the proof of Theorem 4.1.14.

Proof. Let $\psi \in \mathcal{E}$ and $h \in \mathcal{W}_{A}$. Then

$$
\left\|\left(f_{n}(A)-f(A)\right) \psi(A) h(A)\right\|=\left\|\left(\left(f_{n}-f\right) \psi h\right)(A)\right\| \lesssim h\left\|\left(f_{n}-f\right) \psi\right\|_{\mathrm{W}_{v}^{2}\left(\overline{\mathrm{St}_{\omega}}\right)} \stackrel{n \rightarrow \infty}{\longrightarrow} 0 .
$$

As both $\mathcal{E}$ and $\mathcal{W}_{A}$ have dense range, so has $\mathcal{E} \cdot \mathcal{W}_{A}$. As the sequence $\left(f_{n}(A)\right)_{n}$ is uniformly bounded, the claim follows.

We also note the following "weak" convergence lemma.

Theorem 4.2.17 (Weak convergence lemma). Let $A$ be a strip type operator on a Banach space $X$, let $\omega \geq \omega_{\mathrm{st}}(A)$, and let $v: \mathbb{R} \rightarrow[1, \infty)$ be an admissible weight. Suppose that $A$ is densely $(\omega, v)$-Sobolev anchored. Let $f, f_{n} \in \mathrm{C}\left(\overline{\mathrm{St}}_{\omega}\right)$ such that the operators $f(A)$ and $f_{n}(A)$ are defined in the extended auxiliary calculus of $A$ and suppose that the following assertions hold:

1) there is a set $\mathcal{E} \subseteq \mathcal{E}_{v, A}^{2}\left(\overline{\mathrm{St}}_{\omega}\right)$ with total range such that $f \psi, f_{n} \psi \in \mathrm{W}_{v}^{2}\left(\overline{\mathrm{St}}_{\omega}\right)$ and

$$
\psi\left(f_{n}-f\right) \stackrel{n \rightarrow \infty}{\longrightarrow} 0 \quad \text { a.e. on } \mathbb{R}
$$

for all $\psi \in \mathcal{E}$; 
2) $\sup _{n \in \mathbb{R}}\left\|f_{n} \psi\right\|_{\mathrm{W}_{v}^{2}\left(\overline{\mathrm{St}}_{\omega}\right)}<\infty$ for all $\psi \in \mathcal{E}$

3) $\sup _{n \in \mathbb{N}}\left\|f_{n}(A)\right\|<\infty$.

Then, $f(A) \in \mathcal{L}(X)$ and $f_{n}(A) \rightarrow f(A)$ weakly on $X$, i.e.,

$$
\left\langle f_{n}(A) x, x^{\prime}\right\rangle \stackrel{n \rightarrow \infty}{\longrightarrow}\left\langle f(A) x, x^{\prime}\right\rangle
$$

for all $x \in X$ and $x^{\prime} \in X^{\prime}$.

Proof. Fix $x \in X$ and $x^{\prime} \in X^{\prime}$, and let $\psi, \varphi \in \mathcal{E}$ and $h \in \mathcal{W}_{A}$. Set $g_{n}:=\left(f_{n}-f\right) \psi \varphi$ and $\widetilde{g_{n}}:=\mathcal{K}_{\omega, v} g_{n}=v \mathrm{e}^{\omega|\mathbf{s}|} g_{n}^{\vee}$. Then, by Proposition 4.2.9. b), one has

$$
\left\langle\left(f_{n}(A)-f(A)\right) \psi(A) \varphi(A) h(A) x, x^{\prime}\right\rangle=\int_{\mathbb{R}} g_{n}^{\vee}(s)\left\langle h(A, s) x, x^{\prime}\right\rangle \mathrm{d} s .
$$

Due to 2$)$, the sequence $\left(\left(f_{n}-f\right) \psi\right)_{n}$ is uniformly bounded on $\mathbb{R}$. Hence $g_{n} \rightarrow 0$ in $\mathrm{L}^{1}(\mathbb{R})$ and therefore $g_{n}^{\vee} \rightarrow 0$ uniformly on $\mathbb{R}$. As another consequence of 2$)$, the sequence $\left(\widetilde{g_{n}}\right)_{n}$ is bounded in $\mathrm{L}^{2}(\mathbb{R})$. Thus, it follows that $\left(\widetilde{g_{n}}\right)_{n}$ converges weakly to 0 in $\mathrm{L}^{2}(\mathbb{R})$. In particular,

$$
\int_{\mathbb{R}} g_{n}^{\vee}(s)\left\langle h(A, s) x, x^{\prime}\right\rangle \mathrm{d} s=\int_{\mathbb{R}} \widetilde{g_{n}}(s)\left(\frac{\mathrm{e}^{-\omega|s|}}{v(s)}\left\langle h(A, s) x, x^{\prime}\right\rangle\right) \mathrm{d} s \stackrel{n \rightarrow \infty}{\longrightarrow} 0 .
$$

As both $\mathcal{E}$ and $\mathcal{W}_{A}$ have dense range, so has the set $\mathcal{E} \cdot \mathcal{E} \cdot \mathcal{W}_{A}$. Consequently, as the sequence $\left(f_{n}(A)\right)_{n}$ is uniformly bounded, the claim follows readily.

Remark 4.2.18. Consider the special case where $A$ admits a bounded $\mathrm{W}_{v}^{2}\left(\overline{\mathrm{St}}_{\omega}\right)$-calculus and $\left(f_{n}\right)_{n}$ is a bounded sequence in $\operatorname{Hör}_{v}^{2}\left(\overline{\mathrm{St}}_{\omega}\right)$ such that $f_{n} \rightarrow f \in \operatorname{Hör}_{v}^{2}\left(\overline{\mathrm{St}}_{\omega}\right)$ a.e. on $\mathbb{R}$. Then Theorem 4.2.17 yields that $f(A) \in \mathcal{L}(X)$ and $f_{n}(A) \rightarrow f(A)$ weakly on $X$.

\subsection{Results on Bounded Hörmander Calculi}

In this section, we deal with the question of when a strip type operator admits a bounded or even $\gamma$-bounded Hörmander calculus. As bounded Sobolev calculi arise naturally in the following considerations, the notion of a possibly unbounded Sobolev calculus is not needed, and we can leave the more general setting of the previous section behind.

The next theorem is the main result of this thesis. It is a generalization of [17, Theorem 2.2].

Theorem 4.3.1. Let $X$ be a Banach space of type $p \in[1,2]$ and cotype $q \in[2, \infty)$, and let $-\mathrm{i} A$ be the generator of a $C_{0}$-group $\left(U_{s}\right)_{s \in \mathbb{R}}$ on $X$. Suppose there is a measurable function $v_{U}: \mathbb{R} \rightarrow(0, \infty)$ and a constant $\omega \geq 0$ such that

$$
\left\|U_{s}\right\| \leq v_{U}(s) \mathrm{e}^{\omega|s|}
$$

for all $s \in \mathbb{R}$. Further suppose there is $\theta>\omega$ such that $A$ has a bounded $\mathrm{H}^{\infty}\left(\mathrm{St}_{\theta}\right)$ calculus. Let $r \in[1,2]$ with $\frac{1}{r}>\frac{1}{p}-\frac{1}{q}$, and let $v: \mathbb{R} \rightarrow[1, \infty)$ be an admissible weight with $\frac{v_{U}}{v} \in \mathrm{L}^{r}(\mathbb{R})$. Then $A$ has a bounded $\operatorname{Hör}_{v}^{2}\left(\overline{\mathrm{St}}_{\omega}\right)$-calculus. If, in addition, $X$ has Pisier's property $(\alpha)$, then this calculus is $\gamma$-bounded. 
By type and cotype we mean the Rademacher type and cotype of $X$ (see Definition 2.6 .2 .

\section{Remarks 4.3.2.}

1) The growth condition 4.3.1 is quite natural: Recall from Remark 3.3.112) that one has

$$
\left\|\mathrm{e}^{-\mathrm{i} s \mathbf{z}}\right\|_{\operatorname{Hör}_{v}^{2}\left(\overline{\mathrm{St}}_{\omega}\right)} \lesssim v(s) \mathrm{e}^{\omega|\mathbf{s}|}
$$

for all $s \in \mathbb{R}, \omega \geq 0$, and admissible weights $v: \mathbb{R} \rightarrow[1, \infty)$. In particular, if $A$ has a bounded $\operatorname{Hör}_{v}^{2}\left(\overline{\mathrm{St}}_{\omega}\right)$-calculus, one obtains the estimates

$$
\left\|U_{s}\right\|=\left\|\left(\mathrm{e}^{-\mathrm{i} s \mathbf{z}}\right)(A)\right\| \lesssim v(s) \mathrm{e}^{\omega|s|} \quad(s \in \mathbb{R}) .
$$

It is natural to ask, whether the converse is true as well, that is, whether a growth condition like (4.3.1) already leads to a bounded Hörmander calculus.

2) In [17, García-Cuerva, Mauceri, Meda, Sjögren, and Torrea consider the following setting: Let $-B$ be the generator of a symmetric contraction semigroup on some measure space (see Definition 5.2.1, and set $U_{s}:=B^{-\mathrm{i} s}$ for $s \in \mathbb{R}$. Let $\omega \in\left[0, \frac{\pi}{2}\right)$, $\alpha \geq 0$, and $p \in(1, \infty)$, and suppose that

$$
\left\|B^{-i s}\right\| \lesssim(1+|s|)^{\alpha} \mathrm{e}^{\omega|s|}
$$

on $\mathrm{L}^{p}$ for all $s \in \mathbb{R}$. From these estimates they conclude a bounded $\operatorname{Hör}^{\beta, 2}\left(\overline{\mathrm{S}}_{\omega}^{*}\right)$ calculus for $B$ (see Section 4.4 for all $\beta>\alpha+1$ (cf. [17, Theorem 2.2]). This is based on earlier work of Meda (see [41], and see also [11]).

There is a noticable loss of information between the estimates $(4.3 .2)$ and the order of the obtained calculus given in form of a gap of size one between $\alpha$ and $\beta$. The reason for this gap is that the proof in [17] relies on the integrability condition

$$
\frac{(1+|\mathbf{s}|)^{\alpha}}{(1+|\mathbf{s}|)^{\beta}}=\frac{1}{(1+|\mathbf{s}|)^{\beta-\alpha}} \in \mathrm{L}^{1}(\mathbb{R})
$$

In [36], Kriegler and Weis narrowed this gap for the special case $\omega=0$. They showed that in the setting above it suffices to require

$$
\frac{1}{(1+|\mathbf{s}|)^{\beta-\alpha}}=\frac{v_{\mathrm{BIP}}}{(1+|\mathbf{s}|)^{\beta}} \in \mathrm{L}^{2}(\mathbb{R})
$$

which reduces the gap between $\alpha$ and $\beta$ to the size $\frac{1}{2}$. Moreover, Kriegler and Weis showed that the obtained calculus is even $\gamma$-bounded (see [36, Theorem 6.1.(2)]). Aside from the restriction that $\omega=0$, Kriegler and Weis's considerations were also more general as they only considered 0 -sectorial operators with bounded $\mathrm{H}^{\infty}$ calculus and polynomially bounded imaginary powers (plus certain geometric conditions on the underlying Banach space).

Theorem 4.3.1 carries Kriegler and Weis's improvements over to the case where $\omega \neq 0$. In fact, Theorem 4.3.1 tells even a little bit more in the setting considered 
by García-Cuerva et al. From 4.3.2 one can derive a $\gamma$-bounded Hör ${ }_{v}^{2}\left(\overline{\mathrm{S}}_{\omega}^{*}\right)$-calculus for all admissible weights $v$ with

$$
\frac{(1+|\mathbf{s}|)^{\alpha}}{v} \in \mathrm{L}^{2}(\mathbb{R})
$$

In particular, one may choose

$$
v=(1+|\mathbf{s}|)^{\alpha+\frac{1}{2}}(\ln (\mathrm{e}+|\mathbf{s}|))^{\beta} \quad(\beta>1)
$$

or

$$
v=(1+|\mathbf{s}|)^{\alpha+\frac{1}{2}} \ln (\mathrm{e}+|\mathbf{s}|)(\ln (\mathrm{e} \ln (\mathrm{e}+|\mathbf{s}|)))^{\beta} \quad(\beta>1)
$$

and so on. Hence, compared to known results, the order of the calculus can still be improved by logarithmic factors.

However, the result of Kriegler and Weis remains more general in one point: They also study Hörmander spaces based on Sobolev spaces $\mathrm{W}^{\alpha, p}(\mathbb{R})$ for powers $p \neq 2$. This is not present in our theory.

The proof of Theorem 4.3.1 is based on the methods used by Kriegler and Weis in [36] - [36, Theorem 5.1, Corollary 5.2, and Theorem 6.2, resp. Theorem 10.2] to be more precise. The main idea is that, under consideration of the geometry of the underlying Banach space, one can infer a bounded or even $\gamma$-bounded Hörmander calculus from the $\gamma$-boundedness of certain operator families. A precise formulation is given in Proposition 4.3.5, which is the main tool for the proof of Theorem 4.3.1. As preparation for the proof of Proposition 4.3.5, we first characterize all densely defined strip type operators with bounded or even $\gamma$-bounded Hörmander calculus.

\section{Characterization of $(\gamma-)$ Bounded Hörmander Calculi}

From Theorem 4.1 .14 we obtain a characterization of all densely defined strip type operators with a bounded $\operatorname{Hör}_{v}^{2}\left(\overline{\mathrm{St}}_{\omega}\right)$-calculus. Note the similarities to [10, Corollary $2.2]$.

Theorem 4.3.3. Let $A$ be a densely defined strip type operator on some Banach space, let $\theta>\omega \geq \omega_{\text {st }}(A)$, and let $v: \mathbb{R} \rightarrow[1, \infty)$ be an admissible weight. Then the following statements are equivalent:

(i) The operator $A$ admits a bounded $\operatorname{Hör}_{v}^{2}\left(\overline{\mathrm{St}}_{\omega}\right)$-calculus.

(ii) There is a constant $c \geq 0$ such that

$$
\|\psi(A)\| \leq c\|\psi\|_{\mathrm{Hör}_{v}^{2}\left(\overline{\mathrm{St}}_{\omega}\right)}
$$

for all $\psi \in \mathrm{H}_{0}^{\infty}\left(\mathrm{St}_{\theta}\right)$.

Proof. The implication (i) $\Rightarrow$ (ii) is trivial. Suppose that (ii) holds. Then, in particular, $A$ has a bounded $\mathrm{W}_{v}^{2}\left(\overline{\mathrm{St}}_{\omega}\right)$-calculus. Now the claim follows from Lemma 4.1 .16 and Theorem 4.1.14. 
The next result characterizes all densely defined strip type operators with a $\gamma$ bounded Hörmander calculus.

Theorem 4.3.4. Let $A$ be a densely defined strip type operator on some Banach space, let $\theta>\omega \geq \omega_{\mathrm{st}}(A)$, and let $v: \mathbb{R} \rightarrow[1, \infty)$ be an admissible weight. Then the following statements are equivalent:

(i) The operator $A$ admits a $\gamma$-bounded $\operatorname{Hör}_{v}^{2}\left(\overline{\operatorname{St}}_{\omega}\right)$-calculus.

(ii) The set

$$
\left\{\psi(A) \mid \psi \in \mathrm{H}_{0}^{\infty}\left(\mathrm{St}_{\theta}\right),\|\psi\|_{\mathrm{Hör}_{v}^{2}\left(\overline{\mathrm{St}}_{\omega}\right)} \leq 1\right\}
$$

is $\gamma$-bounded.

Proof. We only need to show that (ii) implies (i). As $\gamma$-boundedness implies boundedness in $\mathcal{L}(X)$ we conclude from Theorem 4.3 .3 that $A$ admits a bounded $\mathrm{Hör}_{v}^{2}\left(\mathrm{St}_{\omega}\right)$-calculus. Now choose $K>0$ as in Lemma 4.1.16. Then, since $A$ has a bounded $\operatorname{Hör}_{v}^{2}\left(\overline{\mathrm{St}}_{\omega}\right)$-calculus, the convergence lemma Theorem 4.1.14 yields that each $f(A)$ with $\|f\|_{\operatorname{Hör}_{v}^{2}\left(\overline{\operatorname{St}}_{\omega}\right)} \leq 1$ lies in the strong closure of the set

$$
\mathcal{E}_{K}:=\left\{\psi \in \mathrm{H}_{0}^{\infty}\left(\mathrm{St}_{\theta}\right) \mid\|\psi\|_{\mathrm{Hör}_{v}^{2}\left(\overline{\mathrm{St}_{\omega}}\right)} \leq K\right\} .
$$

As $\mathcal{E}_{K}$ is $\gamma$-bounded as well, so is its closure in the strong operator topology (Theorem 2.6.6. This yields the claim.

\section{Main Tool for the Proof of Theorem 4.3.1}

The following proposition is essentially an extension of [36, Corollary 5.2].

Proposition 4.3.5. Let $A$ be a densely defined strip type operator on a Banach space $X$, let $\theta>\omega \geq \omega_{\mathrm{st}}(A)$, let $v: \mathbb{R} \rightarrow[1, \infty)$ be an admissible weight, and suppose that $A$ has a bounded $\mathrm{H}^{\infty}\left(\mathrm{St}_{\theta}\right)$-calculus.

a) Suppose that $X$ is of finite cotype and that

$$
\llbracket\left(\tau_{n} \psi \cdot f\right)(A) \mid n \in \mathbb{Z} \rrbracket_{\gamma} \lesssim \psi\|f\|_{\operatorname{Hör}_{v}^{2}\left(\overline{\mathrm{St}}_{\omega}\right)}
$$

for all $\psi, f \in \mathrm{H}_{0}^{\infty}\left(\mathrm{St}_{\theta}\right)$. Then $A$ has a bounded $\mathrm{Hör}_{v}^{2}\left(\overline{\mathrm{St}}_{\omega}\right)$-calculus.

b) Suppose that $X$ has Pisier's property $(\alpha)$ and that

$$
\llbracket\left(\tau_{n} \psi \cdot f_{k}\right)(A) \mid n \in \mathbb{Z}, 1 \leq k \leq N \rrbracket_{\gamma} \lesssim \max _{1 \leq k \leq N}\left\|f_{k}\right\|_{\operatorname{Hör}_{v}^{2}\left(\overline{\operatorname{St}}_{\omega}\right)}
$$

for all $\psi, f_{1}, \ldots, f_{N} \in \mathrm{H}_{0}^{\infty}\left(\mathrm{St}_{\theta}\right)$. Then $A$ has a $\gamma$-bounded $\operatorname{Hör}_{v}^{2}\left(\overline{\mathrm{St}}_{\omega}\right)$-calculus.

The proof of Proposition 4.3.5 makes use of the following Paley-Littlewood type result. 
Lemma 4.3.6. Let $A$ be a strip type operator on a Banach space $X$ of finite cotype, and suppose there is $\theta>0$ such that $A$ has a bounded $\mathrm{H}^{\infty}\left(\mathrm{St}_{\theta}\right)$-calculus. Let $\psi_{0}, \psi_{1} \in$ $\mathrm{H}_{0}^{\infty}\left(\mathrm{St}_{\theta}\right)$ such that there is $\psi_{2} \in \mathrm{H}_{0}^{\infty}\left(\mathrm{St}_{\theta}\right)$ with

$$
\sum_{n=-\infty}^{\infty} \psi_{0}(z-n) \psi_{1}(z-n) \psi_{2}(z-n)=1
$$

for all $z \in \mathrm{St}_{\theta}$. Then the following norm equivalence holds on $X$ :

$$
\begin{aligned}
\|x\| & \approx \sup _{N \in \mathbb{N}}\left(\mathbb{E}\left\|\sum_{n=-N}^{N} \gamma_{n} \psi_{0}(A-n) x\right\|^{2}\right)^{1 / 2} \\
& \approx \sup _{N \in \mathbb{N}}\left(\mathbb{E}\left\|\sum_{n=-N}^{N} \gamma_{n} \psi_{1}(A-n) \psi_{0}(A-n) x\right\|^{2}\right)^{1 / 2}
\end{aligned}
$$

where $\psi_{k}(A-n):=\left(\tau_{n} \psi_{k}\right)(A)$ for $k=0,1$ and $n \in \mathbb{Z}$.

Proof. We show that the map

$$
\mathrm{H}_{0}^{\infty}\left(\mathrm{St}_{\theta}\right) \rightarrow \mathrm{H}^{\infty}\left(\mathrm{St}_{\theta} ; \ell^{1}(\mathbb{Z})\right), \quad \psi \mapsto\left[z \mapsto(\psi(z-n))_{n \in \mathbb{Z}}\right]
$$

is well defined. Then, the claim may be concluded from [21, Theorem 4.1] and [21, Corollary 3.10]. Fix $\psi \in \mathrm{H}_{0}^{\infty}\left(\mathrm{St}_{\theta}\right)$ and $z \in \mathrm{St}_{\theta}$. Choose $n_{z} \in \mathbb{Z}$ with $\left|\operatorname{Re} z-n_{z}\right|<1$, and let $c>0$ such that $|\psi| \lesssim \mathrm{e}^{-c|\operatorname{Re} \mathbf{z}|}$. Then

$$
\sum_{n \in \mathbb{Z}}|\psi(z-n)| \lesssim \sum_{n \in \mathbb{Z}} \mathrm{e}^{-c|\operatorname{Re} z-n|} \leq \sum_{n \in \mathbb{Z}} \mathrm{e}^{c} \mathrm{e}^{-c\left|n_{z}-n\right|}=\mathrm{e}^{c} \sum_{n \in \mathbb{Z}} \mathrm{e}^{-c|n|}=\frac{\mathrm{e}^{c}\left(\mathrm{e}^{c}+1\right)}{\mathrm{e}^{c}-1}
$$

Hence,

$$
\sup _{z \in \mathrm{St}_{\theta}}\left\|(\psi(z-n))_{n \in \mathbb{Z}}\right\|_{1} \lesssim \frac{\mathrm{e}^{c}\left(\mathrm{e}^{c}+1\right)}{\mathrm{e}^{c}-1}<\infty .
$$

As the holomorphy of the map

$$
\mathrm{St}_{\theta} \rightarrow \ell^{1}(\mathbb{Z}), \quad z \mapsto(\psi(z-n))_{n \in \mathbb{Z}}
$$

is clear, we conclude the proof.

\section{Remarks 4.3.7.}

1) To see that there exist functions $\psi_{0}, \psi_{1}, \psi_{2} \in \mathrm{H}_{0}^{\infty}\left(\mathrm{St}_{\theta}\right)$ as in the hypothesis of Lemma 4.3.6, one can argue as follows: First, choose a function $\varphi \in \mathrm{H}_{0}^{\infty}\left(\mathrm{St}_{\theta}\right)$ with $\varphi\left(\mathrm{St}_{\theta}\right) \subseteq \mathrm{S}_{\omega}$ for some angle $\omega \in\left(0, \frac{\pi}{2}\right]$ and such that

$$
\int_{\mathbb{R}} \varphi(s) \mathrm{d} s=1 .
$$


(One can consider $\varphi:=\alpha(\cosh (\beta \mathbf{z}))^{-1}$ for suitable constants $\alpha, \beta>0$.) Then

$$
\psi(z):=\int_{0}^{1} \varphi(s-z) \mathrm{d} s \quad\left(z \in \mathrm{St}_{\theta}\right)
$$

defines a function in $\mathrm{H}_{0}^{\infty}\left(\mathrm{St}_{\theta}\right)$ with

$$
\sum_{n=-\infty}^{\infty} \psi(z-n)=\sum_{n=-\infty}^{\infty} \int_{n}^{n+1} \varphi(s-z) \mathrm{d} s=\int_{\mathbb{R}} \varphi(s-z) \mathrm{d} s=1
$$

for each $z \in \mathrm{St}_{\theta}$. As $\varphi\left(\mathrm{St}_{\theta}\right) \subseteq \mathrm{S}_{\omega}$, one has $\psi\left(\mathrm{St}_{\theta}\right) \subseteq \overline{\mathrm{S}}_{\omega}$. Hence, by the open mapping theorem for holomorphic functions, $\psi\left(\mathrm{St}_{\theta}\right) \subseteq \mathrm{S}_{\omega}$. Therefore, one may choose $\psi_{k}:=\psi^{\frac{1}{3}}$ for $k=0,1,2$.

2) Let $X$ be a Banach space, let a sequence $\left(x_{n}\right)_{n}$ in $X$, and independent Gaussians $\left(\gamma_{n}\right)_{n}$. Then, as a consequence of [31, Proposition 6.1.5], the sequence

$$
\left(\mathbb{E}\left\|\sum_{n=0}^{N} \gamma_{n} x_{n}\right\|^{2}\right)_{N \in \mathbb{N}}
$$

is increasing. Therefore, the suprema in Lemma 4.3 .6 can be exchanged with limits.

Proof of Proposition 4.3.5. We prove statement b) first. Let $f_{1}, \ldots, f_{N} \in \mathrm{H}_{0}^{\infty}\left(\mathrm{St}_{\theta}\right)$, and choose $\psi_{0}, \psi_{1} \in \mathrm{H}_{0}^{\infty}\left(\mathrm{St}_{\theta}\right)$ as in Lemma 4.3.6. Let $x_{1}, \ldots, x_{N} \in X$, and observe that

$$
\begin{aligned}
& \left(\mathbb{E}\left\|\sum_{k=1}^{N} \gamma_{k} f_{k}(A) x_{k}\right\|^{2}\right)^{\frac{1}{2}} \\
& \left(\mathbb{E}\left(\sup _{M \in \mathbb{N}} \mathbb{E}^{\prime}\left\|\sum_{m=-M}^{M} \sum_{k=1}^{N} \gamma_{m}^{\prime} \gamma_{k} \psi_{1}(A-m) \psi_{0}(A-m) f_{k}(A) x_{k}\right\|^{2}\right)\right)^{\frac{1}{2}} \\
= & \sup _{M \in \mathbb{N}}\left(\mathbb{E} \mathbb{E}^{\prime}\left\|\sum_{m=-M}^{M} \sum_{k=1}^{N} \gamma_{m}^{\prime} \gamma_{k}\left(\tau_{m} \psi_{1} \cdot f_{k}\right)(A) \psi_{0}(A-m) x_{k}\right\|^{2}\right)^{\frac{1}{2}} \\
\approx & \sup _{M \in \mathbb{N}}\left(\mathbb{E}\left\|\sum_{m=-M}^{M} \sum_{k=1}^{N} \gamma_{m, k}\left(\tau_{m} \psi_{1} \cdot f_{k}\right)(A) \psi_{0}(A-m) x_{k}\right\|^{2}\right)^{\frac{1}{2}} \\
\lesssim & \max _{1 \leq n \leq N}\left\|f_{n}\right\|_{\operatorname{Hör}_{v}^{2}\left(\overline{\operatorname{St}_{\omega}}\right)} \cdot \sup _{M \in \mathbb{N}}\left(\mathbb{E}\left\|\sum_{m=-M}^{M} \sum_{k=1}^{N} \gamma_{m, k} \psi_{0}(A-m) x_{k}\right\|^{2}\right)^{\frac{1}{2}} \\
& \max _{1 \leq n \leq N}\left\|f_{n}\right\|_{\operatorname{Hör}_{v}^{2}\left(\overline{\operatorname{St}}_{\omega}\right)} \cdot\left(\mathbb{E}\left\|\sum_{k=1}^{N} \gamma_{k} x_{k}\right\|^{2}\right)^{\frac{1}{2}} \cdot
\end{aligned}
$$


Here, Lemma 4.3.6 was applied in the second and sixth line, the monotone convergence theorem together with Remark 4.3.7.2) in the third line, property $(\alpha)$ in the fourth and sixth line, and the hypothesis in the fifth line. It follows that the set

$$
\left\{\psi(A) \mid \psi \in \mathrm{H}_{0}^{\infty}\left(\mathrm{St}_{\theta}\right),\|\psi\|_{\mathrm{Hör}_{v}^{2}\left(\overline{\mathrm{St}}_{\omega}\right)} \leq 1\right\}
$$

is $\gamma$-bounded, whence the claim follows from Theorem 4.3.4. For the proof of a) repeat the estimate from above, except only consider the case $N=1$ and appeal to Theorem 4.3 .3 instead (this does not require property $(\alpha)$ ).

As a corollary of Proposition 4.3.5 we obtain a partial generalization of [36, Theorem $5.1]$ :

Theorem 4.3.8. Let $A$ be a densely defined strip type operator on a Banach space $X$, let $\omega \geq \omega_{\mathrm{st}}(A)$, let $v: \mathbb{R} \rightarrow[1, \infty)$ be an admissible weight, and suppose that $A$ has a bounded $\mathrm{H}^{\infty}\left(\mathrm{St}_{\theta}\right)$-calculus for some width $\theta>\omega$.

a) Suppose that $X$ is of finite cotype and that $A$ has a $\gamma$-bounded $\mathrm{W}_{v}^{2}\left(\overline{\mathrm{St}}_{\omega}\right)$-calculus. Then $A$ has a bounded $\operatorname{Hör}_{v}^{2}\left(\overline{\mathrm{St}}_{\omega}\right)$-calculus.

b) Suppose that $X$ has Pisier's property $(\alpha)$. Then $A$ has a $\gamma$-bounded $\operatorname{Hör}_{v}^{2}\left(\overline{\operatorname{St}}_{\omega}\right)$ calculus if and only if $A$ has a $\gamma$-bounded $\mathrm{W}_{v}^{2}\left(\overline{\mathrm{St}}_{\omega}\right)$-calculus.

Proof. Note that if $A$ has a $\gamma$-bounded $\mathrm{W}_{v}^{2}\left(\overline{\mathrm{St}}_{\omega}\right)$-calculus, then

$$
\begin{aligned}
\llbracket\left(\tau_{n} \psi \cdot f_{k}\right)(A) \mid n \in \mathbb{Z}, 1 \leq k \leq N \rrbracket & \lesssim \max _{1 \leq k \leq N} \sup _{n \in \mathbb{Z}}\left\|\tau_{n} \psi \cdot f_{k}\right\|_{\mathrm{W}_{v}^{2}\left(\overline{\mathrm{St}}_{\omega}\right)} \\
& \lesssim \psi \max _{1 \leq k \leq N}\left\|f_{k}\right\|_{\operatorname{Hör}_{v}^{2}\left(\overline{\mathrm{St}}_{\omega}\right)} .
\end{aligned}
$$

Hence, applying Proposition 4.3.5 yields a) and the reverse implication of b). Since a $\gamma$ bounded $\mathrm{Hör}_{v}^{2}\left(\overline{\mathrm{St}}_{\omega}\right)$-calculus clearly implies a $\gamma$-bounded $\mathrm{W}_{v}^{2}\left(\overline{\mathrm{St}}_{\omega}\right)$-calculus, we conclude the proof.

\section{Proof of the Main Result}

The following proof is based on the proof of [36, Theorem 6.1.(2)].

Proof of Theorem 4.3.1. Fix $\psi \in \mathrm{H}_{0}^{\infty}\left(\mathrm{St}_{\theta}\right)$. We show that there is a constant $c_{\psi} \geq 0$ with

$$
\llbracket\left(\tau_{n} \psi \cdot f_{k}\right)(A) \mid n \in \mathbb{Z}, 1 \leq k \leq N \rrbracket_{\gamma} \leq c_{\psi} \max _{1 \leq k \leq N}\left\|f_{k}\right\|_{\operatorname{Hör}_{v}^{2}\left(\overline{\mathrm{St}}_{\omega}\right)}
$$

for all $f_{1}, \ldots, f_{N} \in \mathrm{H}_{0}^{\infty}\left(\mathrm{St}_{\theta}\right)$. Then the claim may be concluded from Proposition 4.3.5 To this end, fix $f_{1}, \ldots, f_{N} \in \mathrm{H}_{0}^{\infty}\left(\mathrm{St}_{\theta}\right)$, and note that

$$
\begin{aligned}
\left(\tau_{n} \psi \cdot f_{m}\right)(A) x & =\int_{\mathbb{R}}\left(\tau_{n} \psi \cdot f_{m}\right)^{\vee}(s) U_{s} x \mathrm{~d} s \\
& =\int_{\mathbb{R}}\left(v(s) \mathrm{e}^{\omega|s|}\left(\tau_{n} \psi \cdot f_{m}\right)^{\vee}(s)\right) \cdot\left(\frac{\mathrm{e}^{-\omega|s|}}{v(s)} U_{s} x\right) \mathrm{d} s
\end{aligned}
$$


for each $n \in \mathbb{Z}, 1 \leq m \leq N$, and $x \in X$. By hypothesis,

$$
\frac{\mathrm{e}^{-\omega|\mathbf{s}|}}{v}\left\|U_{\mathbf{s}}\right\|=\frac{v_{U}}{v} \cdot \frac{\mathrm{e}^{-\omega|\mathbf{s}|}}{v_{U}}\left\|U_{\mathbf{s}}\right\| \in \mathrm{L}^{r}(\mathbb{R})
$$

Hence, once we have shown the estimate

$$
\sup _{n \in \mathbb{Z}}\left(\int_{\mathbb{R}}\left|v(s) \mathrm{e}^{\omega|s|}\left(\tau_{n} \psi \cdot f_{m}\right)^{\vee}(s)\right|^{r^{\prime}} \mathrm{d} s\right)^{\frac{1}{r^{\prime}}} \lesssim \psi\left\|f_{m}\right\|_{\operatorname{Hör}_{v}^{2}\left(\overline{\operatorname{St}}_{\omega}\right)}
$$

for $\frac{1}{r^{\prime}}=1-\frac{1}{r}$, the claim follows from Theorem 2.6.7. For this, note that on one hand

$$
\sup _{n \in \mathbb{Z}}\left(\int_{\mathbb{R}}\left|v(s) \mathrm{e}^{\omega|s|}\left(\tau_{n} \psi \cdot f_{m}\right)^{\vee}(s)\right|^{2} \mathrm{~d} s\right)^{\frac{1}{2}}=\sup _{n \in \mathbb{Z}}\left\|\tau_{n} \psi \cdot f_{m}\right\|_{\mathrm{W}_{v}^{2}\left(\overline{\mathrm{St}}_{\omega}\right)} \lesssim v, \psi\left\|f_{m}\right\|_{\operatorname{Hör}_{v}^{2}\left(\overline{\mathrm{St}}_{\omega}\right)} .
$$

On the other hand,

$$
\sup _{n \in \mathbb{Z}} \sup _{s \in \mathbb{R}}\left|v(s) \mathrm{e}^{\omega|s|}\left(\tau_{n} \psi \cdot f_{m}\right)^{\vee}(s)\right| \lesssim v, \psi\left\|f_{m}\right\|_{\operatorname{Hor}_{v}^{2}\left(\overline{\operatorname{St}_{\omega}}\right)}
$$

by Theorem 3.3.21. As $r^{\prime} \in[2, \infty]$, the estimate 4.3.5 follows by interpolation.

Remark 4.3.9 (Comparison to Kriegler's and Weis's work). Keeping the notation of Theorem 4.3.1. Kriegler and Weis consider the case where $\omega=0$ and $v_{U}=v_{\alpha}:=$ $(1+|\mathbf{s}|)^{\alpha}$ for $\alpha>0$. In the proof of [36, Theorem 6.1] the general aim is to show that the family $\left(\frac{1}{v_{\beta}} U_{s}\right)_{s \in \mathbb{R}}$ is $R\left[\mathrm{~L}^{2}(\mathbb{R})\right]$-bounded for a certain range $\beta>\alpha$. That is, they aim to show that the sets

$$
\left\{\int_{\mathbb{R}} f(s) \frac{1}{v_{\beta}(s)} U_{s} \mathrm{~d} s \mid\|f\|_{2} \leq 1\right\}
$$

are $R$-bounded. This is equivalent to saying that $A$ admits an $R$-bounded $\mathrm{W}^{\beta, 2}(\mathbb{R})$ calculus ([35, Theorem 6.1]). Once an $R$-bounded Sobolev calculus is established, a bounded or even $R$-bounded Hörmander calculus is inferred as in Theorem 4.3.8.

The proof of Theorem 4.3.1 works along the same lines. However, we show that it is enough to verify the $\gamma$-bounds (4.3.4) from Proposition 4.3.5. In a Banach space with property $(\alpha)$ these bounds imply a $\gamma$-bounded Sobolev calculus by Theorem 4.3.8. However, if the underlying Banach space does not have property $(\alpha)$, having the bounds (4.3.4) available is a possibly weaker condition than the presence of a $\gamma$-bounded Sobolev calculus.

Another slight improvement is that we consider a finer scale of Hörmander spaces and thus obtain a finer scale of quality of the respective functional calculi (see Remark 4.3 .2 .

Still, our theory does not cover Hörmander spaces $\operatorname{Hör}^{\alpha, p}(\mathbb{R})$ for other powers $p \neq 2$, while the results of Kriegler and Weis do. 


\section{An Abstract Multiplier Theorem for General Banach Spaces}

The following theorem extends [17, Theorem 2.2] to general Banach spaces. The proof is based on the more classical approach described in [6, p. $944-946]$.

Theorem 4.3.10. Let $-\mathrm{i} A$ be the generator of a $C_{0}$-group $\left(U_{s}\right)_{s \in \mathbb{R}}$ on a Banach space $X$. Suppose there is a measurable function $v_{U}: \mathbb{R} \rightarrow(0, \infty)$ and a constant $\omega \geq 0$ such that

$$
\left\|U_{s}\right\| \leq v_{U}(s) \mathrm{e}^{\omega|s|}
$$

for all $s \in \mathbb{R}$. Further suppose there is $\theta>\omega$ such that $A$ has a bounded $\mathrm{H}^{\infty}\left(\mathrm{St}_{\theta}\right)$ calculus, and let $v: \mathbb{R} \rightarrow[1, \infty)$ be an admissible weight with $\frac{v_{U}}{v} \in \mathrm{L}^{1}(\mathbb{R})$. Then $A$ has a bounded $\operatorname{Hör}_{v}^{2}\left(\overline{\mathrm{St}}_{\omega}\right)$-calculus.

Remark 4.3.11. The main difference between Theorem 4.3.1 and Theorem 4.3.10 is that the latter drops the geometric assumption on the Banach space $X$. This comes at the cost of the requirement that $\frac{v_{U}}{v} \in \mathrm{L}^{1}(\mathbb{R})$.

Proof of Theorem 4.3.10. We may suppose that $\theta>\omega$. Let $f \in \mathrm{H}_{0}^{\infty}\left(\mathrm{St}_{\theta}\right)$. Recall from Lemma 3.3.18 that $f$ admits the representation formula

$$
f(z)=\int_{\mathbb{R}}\left(\tau_{z} \psi \cdot f\right)^{\vee}(s) \mathrm{e}^{-\mathrm{i} s z} \mathrm{~d} s \quad\left(z \in \mathrm{St}_{\theta}\right) .
$$

We establish the identity

$$
f(A)=\int_{\mathbb{R}} F(A, s) U_{s} \mathrm{~d} s
$$

as strong integrals, where $F(\mathbf{z}, s):=\left(\tau_{\mathbf{z}} \psi \cdot f\right)^{\vee}(s)$ and $F(A, s):=F(\mathbf{z}, s)(A)$ for $s \in \mathbb{R}$. To this end, first note that the mapping

$$
\mathbb{C} \rightarrow \mathrm{L}^{1}(\mathbb{R}), \quad z \mapsto \tau_{z} \psi \cdot f,
$$

is entire, hence so is $F(\mathbf{z}, s)$ for every $s \in \mathbb{R}$. Moreover, by Theorem 3.3 .21 .

$$
\|F(\mathbf{z}, s)\|_{\infty, \mathrm{St}_{\theta}} \lesssim \frac{\mathrm{e}^{-\omega|s|}}{v(s)}\|f\|_{\mathrm{Hör}_{v}^{2}\left(\overline{\mathrm{St}}_{\omega}\right)} .
$$

Therefore, $F(A, s)$ is well-defined within the holomorphic calculus of $A$ and

$$
\|F(A, s)\| \lesssim \frac{\mathrm{e}^{-\omega|s|}}{v(s)}\|f\|_{\operatorname{Hör}_{v}^{2}\left(\overline{\mathrm{St}}_{\omega}\right)} .
$$

Now, let $|\operatorname{Im} \lambda|>\omega$ and observe for $\delta \in(\omega, \theta)$,

$$
\begin{aligned}
f(A) R(\lambda, A)^{2} & =\frac{1}{2 \pi \mathrm{i}} \int_{\partial \mathrm{St}_{\delta}} \frac{f(z)}{(\lambda-z)^{2}} R(z, A) \mathrm{d} z \\
& =\frac{1}{2 \pi \mathrm{i}} \int_{\partial \mathrm{St}_{\delta}}\left(\int_{\mathbb{R}}\left(\tau_{z} \psi \cdot f\right)^{\vee}(s) \mathrm{e}^{-\mathrm{i} s z} \mathrm{~d} s\right) \frac{1}{(\lambda-z)^{2}} R(z, A) \mathrm{d} z
\end{aligned}
$$




$$
\begin{aligned}
& =\frac{1}{2 \pi \mathrm{i}} \int_{\partial \mathrm{St}_{\delta}} \int_{\mathbb{R}}\left(\tau_{z} \psi \cdot f\right)^{\vee}(s) \mathrm{e}^{-\mathrm{i} s z} \frac{1}{(\lambda-z)^{2}} R(z, A) \mathrm{d} s \mathrm{~d} z \\
& =\int_{\mathbb{R}}\left(\frac{1}{2 \pi \mathrm{i}} \int_{\partial \mathrm{St}_{\delta}}\left(\tau_{z} \psi \cdot f\right)^{\vee}(s) \mathrm{e}^{-\mathrm{i} s z} \frac{1}{(\lambda-z)^{2}} R(z, A) \mathrm{d} z\right) \mathrm{d} s \\
& =\int_{\mathbb{R}} F(A, s) U_{s} R(\lambda, A)^{2} \mathrm{~d} s \\
& =\left(\int_{\mathbb{R}} F(A, s) U_{s} \mathrm{~d} s\right) R(\lambda, A)^{2} .
\end{aligned}
$$

As $R(\lambda, A)^{2}$ has dense range, one obtains identity 4.3.7. Hence, one can estimate

$$
\begin{aligned}
\|f(A)\| & \leq \int_{\mathbb{R}}\|F(A, s)\|\left\|U_{s}\right\| \mathrm{d} s \\
& \lesssim \int_{R} \frac{\mathrm{e}^{-\omega|s|}}{v(s)}\|f\|_{\operatorname{Hor}_{v}^{2}\left(\overline{\mathrm{St}}_{\omega}\right)}\left\|U_{s}\right\| \mathrm{d} s \\
& \leq\left(\int_{\mathbb{R}} \frac{v_{U}(s)}{v(s)} \mathrm{d} s\right)\|f\|_{\operatorname{Hör}_{v}^{2}\left(\overline{\mathrm{St}}_{\omega}\right)} \\
& =\left\|\frac{v_{U}}{v}\right\|_{1}\|f\|_{\operatorname{Hör}_{v}^{2}\left(\overline{\mathrm{St}}_{\omega}\right)},
\end{aligned}
$$

where (4.3.8) was used in the second line. Now the claim follows from Theorem 4.3.3.

\subsection{The Hörmander Calculus for Sectorial Operators}

Now we give a short discussion on how to define a Hörmander functional calculus for sectorial operators.

Definition 4.4.1. Let $A$ be a sectorial operator on some Banach space, let $\omega_{\text {se }}(A) \leq$ $\omega<\pi$, and let $v: \mathbb{R} \rightarrow[1, \infty)$ be an admissible weight. The operator $A$ has a bounded $\mathrm{W}_{v}^{2}\left(\overline{\mathrm{S}}_{\omega}^{*}\right)$-calculus if there is an angle $\theta \in(\omega, \pi)$ and a constant $c \geq 0$ with

$$
\|\psi(A)\| \leq c\|\psi\|_{\mathrm{W}_{v}^{2}\left(\overline{\mathrm{S}}_{\omega}^{*}\right)}
$$

for all $\psi \in \mathrm{H}_{0}^{\infty}\left(\mathrm{S}_{\theta}\right)$.

Lemma 4.4.2. Let $A, \omega$, and $v$ be given as in Definition 4.4.1, and suppose that $A$ has a bounded $\mathrm{W}_{v}^{2}\left(\overline{\mathrm{S}}_{\omega}^{*}\right)$-calculus. Then the $\mathrm{W}_{v}^{2}\left(\overline{\mathrm{S}}_{\omega}^{*}\right)$-calculus of $A$ is non-degenerate if and only if $A$ is injective.

Proof. Denote the Sobolev calculus of $A$ by $\Phi: \mathrm{W}_{v}^{2}\left(\overline{\mathrm{S}}_{\omega}^{*}\right) \rightarrow \mathcal{L}(X)$. In the proof of [24, Corollary 9.7] it is shown that $\operatorname{ker} A \subseteq \operatorname{ker} \psi(A)$ for each $\psi \in \mathcal{E}\left[\mathrm{S}_{\omega}\right]$. This naturally extends to all functions $f \in \mathrm{W}_{v}^{2}\left(\overline{\mathrm{S}}_{\omega}^{*}\right)$ so that the claim follows readily.

For an injective sectorial operator $A$ the terms extended Sobolev calculus, Hörmander calculus and bounded Hörmander calculus (and all variations of this) 
are defined as in the strip case (with the obvious changes). Again one can show that the extended Sobolev calculus extends the holomorphic calculus of $A$. In particular, we write " $f(A)$ " even if the operator is only defined in the extended Sobolev calculus.

Recall from Theorem 2.4.9 and Theorem 2.4.10 that there is a natural relation between injective sectorial operators and strip type operators. We also have a composition rule for the extended Sobolev calculus.

Theorem 4.4.3 (Composition rule). Let $A$ be an injective sectorial operator, let $\omega_{\mathrm{se}}(A) \leq$ $\omega<\pi$, and let $v: \mathbb{R} \rightarrow[1, \infty)$ be an admissible weight. Further, let $f: \overline{\mathrm{St}}_{\omega} \rightarrow \mathbb{C}$ be a function. Then the following statements hold:

a) The operator $\log A$ has a bounded $\mathrm{W}_{v}^{2}\left(\overline{\mathrm{St}}_{\omega}\right)$-calculus if and only if $A$ has a bounded $\mathrm{W}_{v}^{2}\left(\overline{\mathrm{S}}_{\omega}^{*}\right)$-calculus.

b) Suppose that $A$ has a bounded $\mathrm{W}_{v}^{2}\left(\overline{\mathrm{S}}_{\omega}^{*}\right)$-calculus. Then the operator $f(\log A)$ is defined within the extended $\mathrm{W}_{v}^{2}\left(\overline{\mathrm{St}}_{\omega}\right)$-calculus of $\log A$ if and only if $(f \circ \log )(A)$ is defined within the extended $\mathrm{W}_{v}^{2}\left(\overline{\mathrm{S}}_{\omega}^{*}\right)$-calculus of $A$. In this case,

$$
f(\log A)=(f \circ \log )(A) .
$$

Proof. Let $\theta \in(\omega, \pi)$. From Theorem 2.4.10 we conclude that

$$
\psi(\log A)=(\psi \circ \log )(A)
$$

for all $\psi \in \mathrm{H}_{0}^{\infty}\left(\mathrm{St}_{\theta}\right)$. This easily implies a). Next, fix $f \in \mathrm{W}_{v}^{2}\left(\overline{\mathrm{St}}_{\omega}\right)$ and choose a sequence $\left(\psi_{n}\right)_{n} \in \mathrm{H}_{0}^{\infty}\left(\mathrm{St}_{\theta}\right)$ with $\psi_{n} \rightarrow f$ in $\mathrm{W}_{v}^{2}\left(\overline{\mathrm{St}}_{\omega}\right)$. Then $\left(\psi_{n} \circ \log \right)_{n}$ is a sequence in $\mathrm{H}_{0}^{\infty}\left(\mathrm{St}_{\theta}\right)$ with $\psi_{n} \circ \log \rightarrow f \circ \log$ in $\mathrm{W}_{v}^{2}\left(\overline{\mathrm{S}}_{\omega}^{*}\right)$. Hence,

$$
f(\log (A))=\lim _{n \rightarrow \infty} \psi_{n}(\log A)=\lim _{n \rightarrow \infty}\left(\psi_{n} \circ \log \right)(A)=(f \circ \log )(A),
$$

i.e., the composition rule holds for all Sobolev functions. Finally, the composition rule carries over naturally to Hörmander functions.

With Theorem 4.4.3, the abstract theory of Sobolev and Hörmander functional calculi for strip type operators now carries over to the sectorial case. In particular, one obtains a sectorial version of Theorem 4.3.1.

Theorem 4.4.4. Let $X$ be a Banach space of type $p \in[1,2]$ and cotype $q \in[2, \infty)$, and let $A$ be an injective sectorial operator on $X$ with (BIP). Suppose there is a measurable function $v_{\mathrm{BIP}}: \mathbb{R} \rightarrow(0, \infty)$ and a constant $\omega \in[0, \pi)$ such that

$$
\left\|A^{-\mathrm{i} s}\right\| \leq v_{\operatorname{BIP}}(s) \mathrm{e}^{\omega|s|}
$$

for all $s \in \mathbb{R}$. Further suppose there is $\theta \in(\omega, \pi)$ such that $A$ has a bounded $\mathrm{H}^{\infty}\left(\mathrm{S}_{\theta}\right)$ calculus. Let $r \in[1,2]$ with $\frac{1}{r}>\frac{1}{p}-\frac{1}{q}$, and let $v: \mathbb{R} \rightarrow[1, \infty)$ be an admissible weight with $\frac{v_{\mathrm{BIP}}}{v} \in \mathrm{L}^{r}(\mathbb{R})$. Then $A$ has a bounded $\operatorname{Hör}_{v}^{2}\left(\overline{\mathrm{S}}_{\omega}^{*}\right)$-calculus. If, in addition, $X$ has Pisier's property $(\alpha)$, then the calculus is $\gamma$-bounded. 


\section{The Hörmander Calculus for Non-Injective Sectorial Operators}

For a given non-injective sectorial operator $A$ on a Banach space $X$ there is also a canonical way to define a holomorphic functional calculus (see [24, Chapter 9] for details). However, in the non-injective case there arise natural restrictions on the considered function spaces. In particular, if a function $f: \mathrm{S}_{\omega} \rightarrow \mathbb{C}$ with $\omega \in\left(\omega_{\mathrm{se}}(A), \pi\right)$ is anchored in this calculus, then $f$ necessarily admits a continuous extension to zero. (This is a consequence of [24, Exercise 9.7].) Therefore, the expressions " $\log A$ " and " $A$ "is" (for $s \in \mathbb{R})$ are not covered by the holomorphic calculus of $A$ anymore.

If $X$ is reflexive, one can amend this by playing the holomorphic calculus of $A$ back to that of a suitable injective operator, the so-called injective part of $A$ : Let $X$ be a reflexive Banach space, and let $A$ be a non-injective sectorial operator on $X$. Then $X$ admits the decomposition $X=X_{0} \oplus X_{1}$ where $X_{0}=\operatorname{ker} A$ and $X_{1}=\overline{\operatorname{ran} A}$ ([23, Proposition 2.1.1.h)]). In this case, the operator

$$
A_{1}:=A \cap\left(X_{1} \oplus X_{1}\right),
$$

called the injective part of $A$, is an injective sectorial operator on $X_{1}$ with $\omega_{\text {se }}\left(A_{1}\right) \leq$ $\omega_{\text {se }}(A)([23$, p. 24] $)$. Hence it is possible to take imaginary powers or the logarithm of $A$ at least on the subspace $X_{1}$ by considering the operators $A_{1}^{-\mathrm{is}}$ and $\log A_{1}$.

Moreover, one can show the following: If a function $f: \mathrm{S}_{\omega} \rightarrow \mathbb{C}$ is anchored in the holomorphic calculus of $A$, then $f$ is also anchored in the (classical) holomorphic calculus of $A_{1}$ and one has

$$
\operatorname{dom} f(A)=\operatorname{ker} A \oplus \operatorname{dom} f\left(A_{1}\right)
$$

as well as

$$
f(A) x=f(0) x_{0}+f\left(A_{1}\right) x_{1} \quad\left(x=x_{0}+x_{1} \in \operatorname{dom} f(A)\right)
$$

(see [23, Corollary 2.3.9] for the case, in which $f$ is anchored by one element; for the general case the proof can be adapted accordingly).

For the remainder of this subsection, fix a reflexive Banach space $X$, a non-injective sectorial operator $A$ on $X$, and let $A_{1}$ be the injective part of $A$. In the following, we construct a Hörmander calculus for $A$ with the help of the calculus available for $A_{1}$. Both 4.4.1) and 4.4.2 serve as motivation for this. We start with the following lemma.

Lemma 4.4.5. Let $A$ and $A_{1}$ be given as above, let $\omega \in\left[\omega_{\mathrm{se}}(A), \pi\right)$, and let $v: \mathbb{R} \rightarrow$ $[1, \infty)$ be an admissible weight. Then $A$ has a bounded $\mathrm{W}_{v}^{2}\left(\overline{\mathrm{S}}_{\omega}^{*}\right)$-calculus if and only if $A_{1}$ has bounded $\mathrm{W}_{v}^{2}\left(\overline{\mathrm{S}}_{\omega}^{*}\right)$-calculus.

Proof. Let $\theta \in(\omega, \pi)$, and let $\psi \in \mathrm{H}_{0}^{\infty}\left(\mathrm{S}_{\theta}\right)$. Note that $\psi(z) \rightarrow 0$ as $z \rightarrow 0$. Hence, by (4.4.2),

$$
\|\psi(A)\|=\left\|\psi\left(A_{1}\right)\right\|
$$

The claim follows readily. 
Definition 4.4.6. Let $X, A$, and $A_{1}$ be given as above, let $\omega \in\left[\omega_{\mathrm{se}}(A), \pi\right)$, and let $v: \mathbb{R} \rightarrow[1, \infty)$ be an admissible weight. Set $\mathrm{C}_{\omega}:=\mathrm{C}\left(\overline{\mathrm{S}}_{\omega}\right)$ and $\mathcal{E}:=\mathrm{W}_{v}^{2}\left(\overline{\mathrm{S}}_{\omega}^{*}\right)$. Then for each $f \in\left[\mathrm{C}_{\omega}\right]_{\mathcal{E}}$ we define the operator $f(A)$ by setting

$$
\operatorname{dom} f(A):=\operatorname{ker} A \oplus \operatorname{dom} f\left(A_{1}\right),
$$

and

$$
f(A) x:=f(0) x_{0}+f\left(A_{1}\right) x_{1} \quad\left(x=x_{0}+x_{1} \in \operatorname{dom} f(A)\right) .
$$

The mapping

$$
\left[\mathrm{C}_{\omega}\right]_{\mathcal{E}} \rightarrow \mathcal{C}(X), \quad f \mapsto f(A),
$$

is called the extended $\mathrm{W}_{v}^{2}\left(\overline{\mathrm{S}}_{\omega}^{*}\right)$-calculus (or just extended Sobolev calculus) of $A$.

For the non-injective case we consider the following subclass of Hörmander functions.

Definition 4.4.7. Let $\omega \in[0, \pi)$, and let $v: \mathbb{R} \rightarrow[1, \infty)$ be an admissible weight. Set

$$
\text { Hör }_{v, *}^{2}\left(\overline{\mathrm{S}}_{\omega}^{*}\right):=\left\{f \in \operatorname{Hör}_{v}^{2}\left(\overline{\mathrm{S}}_{\omega}^{*}\right) \mid f(0):=\lim _{z \rightarrow 0} f(z) \text { exists }\right\} \text {. }
$$

We also write $\operatorname{Hör}_{*}^{\alpha, 2}\left(\overline{\mathrm{S}}_{\omega}^{*}\right):=\operatorname{Hör}_{v, *}^{2}\left(\overline{\mathrm{S}}_{\omega}^{*}\right)$ if $v=(1+|\mathbf{s}|)^{\alpha}$ for some power $\alpha>\frac{1}{2}$.

Remark 4.4.8. Note that $\operatorname{Hör}_{v, *}^{2}\left(\overline{\mathrm{S}}_{\omega}^{*}\right)$ is a closed subalgebra of Hör ${ }_{v}^{2}\left(\overline{\mathrm{S}}_{\omega}^{*}\right)$ and continuously embeds into the space $\mathrm{C}_{\mathrm{b}}\left(\overline{\mathrm{S}}_{\omega}\right)$.

Now we arrive at a Hörmander type calculus for non-injective sectorial operators.

Definition 4.4.9. Let $X, A$, and $A_{1}$ be given as above, let $\omega \in\left[\omega_{\mathrm{se}}(A), \pi\right)$, and let $v: \mathbb{R} \rightarrow[1, \infty)$ be an admissible weight. The restriction of the extended Sobolev calculus of $A$ to $\mathrm{Hör}_{v, *}^{2}\left(\overline{\mathrm{S}}_{\omega}^{*}\right)$ is called the $\mathrm{Hör}_{v, *}^{2}\left(\overline{\mathrm{S}}_{\omega}^{*}\right)$-calculus of $A$, sometimes just Hörmander calculus of $A$. If $f(A) \in \mathcal{L}(X)$ for all $f \in \operatorname{Hör}_{v, *}^{2}\left(\overline{\mathrm{S}}_{\omega}^{*}\right)$, we also say that $A$ has a bounded $\mathrm{Hör}_{v, *}^{2}\left(\overline{\mathrm{S}}_{\omega}^{*}\right)$-calculus.

We close with the following proposition on $(\gamma$-)bounded Hörmander calculi for the non-injective case.

Proposition 4.4.10. In the situation of Definition 4.4.9 the following statements hold:

a) If $A_{1}$ has a bounded $\operatorname{Hör}_{v}^{2}\left(\overline{\mathrm{S}}_{\omega}^{*}\right)$-calculus, then $A$ has a bounded $\mathrm{Hör}_{v, *}^{2}\left(\overline{\mathrm{S}}_{\omega}^{*}\right)$-calculus.

b) If $A_{1}$ has a $\gamma$-bounded $\mathrm{Hör}_{v}^{2}\left(\overline{\mathrm{S}}_{\omega}^{*}\right)$-calculus, then $A$ has a $\gamma$-bounded $\mathrm{Hör}_{v, *}^{2}\left(\overline{\mathrm{S}}_{\omega}^{*}\right)$ calculus.

Proof. Statement a) is immediate. For b) we want to appeal to Proposition 2.6.11. To this end, first note that the restriction of the $\operatorname{Hör}_{v}^{2}\left(\overline{\mathrm{S}}_{\omega}^{*}\right)$-calculus of $A_{1}$ to $\operatorname{Hör}_{v, *}^{2}\left(\overline{\mathrm{S}}_{\omega}^{*}\right)$ is still $\gamma$-bounded. Hence, we only need to show that the mapping $\Phi_{0}: \operatorname{Hör}_{v, *}^{2}\left(\overline{\mathrm{S}}_{\omega}^{*}\right) \rightarrow \mathcal{L}\left(X_{0}\right)$, defined by

$$
\Phi_{0}(f) x_{0}:=f(0) x_{0} \quad\left(f \in \operatorname{Hör}_{v, *}^{2}\left(\overline{\mathrm{S}}_{\omega}^{*}\right), x_{0} \in X_{0}\right),
$$

is $\gamma$-bounded. But this already follows from Theorem 2.6.10 and the fact that Hör ${ }_{v, *}\left(\overline{\mathrm{S}}_{\omega}^{*}\right)$ continuously embeds into $\mathrm{C}_{\mathrm{b}}\left(\overline{\mathrm{S}}_{\omega}\right)$. 


\section{Chapter 5}

\section{Applications}

In this chapter, we give three applications of Theorem 4.4.4. In Section 5.1, elliptic partial differential operators with constant coefficients are treated. In Section 5.2 , we take a look at the classical setting for abstract multiplier theorems and prove a multiplier result for generators of symmetric contraction semigroups. This is an improvement of [6, Theorem 1]. Finally, in Section 5.3, a multiplier result for the Ornstein-Uhlenbeck operator is proved.

\subsection{Elliptic Partial Differential Operators with Constant Coefficients}

Throughout this section, let $d \in \mathbb{N}$. We recall the definition of a homogeneous elliptic partial differential operator with constant coefficients on $\mathrm{L}^{p}\left(\mathbb{R}^{d}\right)$. These operators are associated to a certain class of polynomials.

Definition 5.1.1. Let $m \in \mathbb{N}$, and let $a: \mathbb{R}^{d} \rightarrow \mathbb{C}$ be a polynomial which is homogeneous of degree $m$, i.e.,

$$
a=\sum_{|\alpha|=m} a_{\alpha} \mathrm{i}^{|\alpha|} \mathbf{x}^{\alpha}
$$

for some coefficients $a_{\alpha} \in \mathbb{C}$ where $\alpha \in \mathbb{N}_{0}^{d}$ with $|\alpha|=m$. The polynomial $a$ is called strongly elliptic if $-1 \notin a\left(\mathbb{R}^{d}\right)$ and if there is a constant $c>0$ with $\operatorname{Re} a \geq c|\mathbf{x}|^{m}$ on $\mathbb{R}^{d}$.

Strongly elliptic polynomials map $\mathbb{R}^{d}$ into sectors (cf. [24, Lemma 12.3]).

Lemma 5.1.2. Let $a: \mathbb{R}^{d} \rightarrow \mathbb{C}$ be a strongly elliptic homogeneous polynomial. Then $a(x)=0$ if and only if $x=0$, and there is an angle $\omega \in\left[0, \frac{\pi}{2}\right)$ such that $\overline{a\left(\mathbb{R}^{d}\right)} \subseteq \overline{\mathrm{S}}_{\omega}$.

Definition 5.1.3. Let $a: \mathbb{R}^{d} \rightarrow \mathbb{C}$ be a strongly elliptic polynomial. Set

$$
\omega_{a}:=\inf \left\{\omega \in\left(0, \frac{\pi}{2}\right) \mid \overline{a\left(\mathbb{R}^{d}\right)} \subseteq \overline{\mathrm{S}}_{\omega}\right\}
$$


Definition 5.1.4. Let $a: \mathbb{R}^{d} \rightarrow \mathbb{C}$ be a strongly elliptic polynomial of the form

$$
a=\sum_{|\alpha|=m} a_{\alpha} \mathrm{i}^{|\alpha|} \mathbf{x}^{\alpha} .
$$

To the function $a$ one associates the operator

$$
\mathcal{A}:=\left\{(f, g) \in \mathcal{S}\left(\mathbb{R}^{d}\right) \oplus \mathcal{S}\left(\mathbb{R}^{d}\right) \mid g=\sum_{|\alpha|=m} a_{\alpha} \mathrm{D}^{\alpha} f\right\}
$$

and writes $a \sim \mathcal{A}$. Here, $\mathcal{S}\left(\mathbb{R}^{d}\right)$ denotes the Schwartz space on $\mathbb{R}^{d}$.

For the proof of the next theorem, see [24, Theorem 12.7].

Theorem 5.1.5. Let $a: \mathbb{R}^{d} \rightarrow \mathbb{C}$ be a strongly elliptic polynomial, and let $a \sim \mathcal{A}$. Let $p \in(1, \infty)$. Then $\mathcal{A}$ is closable in $\mathrm{L}^{p}\left(\mathbb{R}^{d}\right)$, i.e.,

$$
A_{p}:=\overline{\mathcal{A}}^{\|\cdot\|_{\mathrm{L}^{p}\left(\mathbb{R}^{d}\right)}}
$$

is a linear operator. Moreover, $A_{p}$ is a sectorial operator with $\omega_{\mathrm{se}}\left(A_{p}\right) \leq \omega_{a}$, the set

$$
\operatorname{dom} A_{p} \cap \operatorname{ran} A_{p}
$$

is dense in $\mathrm{L}^{p}\left(\mathbb{R}^{d}\right)$, and $A_{p}$ has a bounded $\mathrm{H}^{\infty}\left(\mathrm{S}_{\theta}\right)$-calculus for each $\theta \in\left(\omega_{a}, \pi\right)$.

\section{Remarks 5.1.6.}

1) Each sectorial operator with dense range is already injective (see [23, Proposition 2.1.1.d)]).

2) Let $A$ be a sectorial operator on a Banach space $X$ such that $\operatorname{dom} A \cap \operatorname{ran} A$ is dense in $X$. In this case, one often says that $A$ has dense domain and range. If $A$ has dense domain and range and $A$ also admits a bounded $\mathrm{H}^{\infty}$-calculus, then $A$ has the (BIP) property. This follows from [23, Corollary 3.5.7].

Next, we estimate the growth of the imaginary powers of $A_{p}$.

Lemma 5.1.7. In the situation of Theorem 5.1.5, there is a constant $c \geq 0$ only dependent on $a, d$, and $p$ such that

$$
\left\|A_{p}^{-\mathrm{i} s}\right\| \leq c(1+|s|)^{k_{d}} \mathrm{e}^{\omega_{a}|s|}
$$

for all $s \in \mathbb{R}$, where $k_{d}:=\left\lfloor\frac{d}{2}\right\rfloor+1$.

Proof. Fix $s \in \mathbb{R}$. By the Mikhlin multiplier theorem (cf. [19, Theorem 5.2.7.a)]), one has

$$
\left\|A_{p}^{-\mathrm{i} s}\right\| \lesssim_{p} \max _{|\alpha| \leq k_{d}}\left\||\mathbf{x}|^{|\alpha|} \cdot \mathrm{D}^{\alpha}\left(a^{-\mathrm{i} s}\right)\right\|_{\infty, \mathbb{R}^{d} \backslash\{0\}} .
$$


Suppose that $a$ is homogeneous of degree $m \in \mathbb{N}$. Following the proof of [24, Lemma 12.6], we write

$$
D^{\alpha}\left(a^{-\mathrm{i} s}\right)(x)=\sum_{k=0}^{|\alpha|}(-1)^{k-1}\left(\prod_{j=0}^{k-1}(\mathrm{i} s+k)\right) a^{-\mathrm{i} s-k}(x) p_{\alpha, k}(x)
$$

for $x \neq 0$, where $p_{\alpha, k}=0$ if $k m<|\alpha|$, and $p_{\alpha, k}$ is a homogeneous polynomial of degree $k m-|\alpha|$ otherwise. In any case, one has

$$
\left|p_{\alpha, k}\right| \lesssim p_{\alpha, k}|\mathbf{x}|^{k m-|\alpha|}
$$

Hence,

$$
\left|a^{-k}(x) p_{\alpha, k}(x)\right| \lesssim_{a, p_{\alpha, k}}|x|^{-k m}|x|^{k m-|\alpha|}=|x|^{-|\alpha|}
$$

for each $x \neq 0$. Therefore, using identity (5.1.1), one obtains

$$
\max _{|\alpha| \leq k_{d}}\left\||\mathbf{x}|^{|\alpha|} \cdot \mathrm{D}^{\alpha}\left(a^{-\mathrm{i} s}\right)\right\|_{\infty, \mathbb{R ^ { d }} \backslash\{0\}} \lesssim \sum_{k=0}^{k_{d}}\left|\prod_{j=0}^{k-1}(\mathrm{i} s+k)\right| \cdot\left\|a^{-\mathrm{i} s}\right\|_{\infty, \mathbb{R}^{d} \backslash\{0\}} .
$$

Finally, note that

$$
\left\|a^{-\mathrm{i} s}\right\|_{\infty, \mathbb{R}^{d}} \leq \mathrm{e}^{\omega_{a}|s|}
$$

and that

$$
\left|\prod_{j=0}^{k-1}(\mathrm{i} s+k)\right| \lesssim_{d}(1+|s|)^{k_{d}}
$$

for all $k \leq k_{d}$. Plugging the estimates (5.1.3) and (5.1.4) into (5.1.2) yields the claim.

Now we want to apply Theorem 4.4.4 to obtain a bounded $\operatorname{Hör}_{v}^{2}\left(\overline{\mathrm{S}}_{\omega_{a}}\right)$-calculus for $A_{p}$. To this end, recall that each scalar $\mathrm{L}^{p}$-space has type $\min \{2, p\}$ and cotype $\max \{2, p\}$. Moreover,

$$
\frac{1}{\min \{2, p\}}-\frac{1}{\max \{2, p\}}<\frac{1}{2}
$$

for each $p \in(1, \infty)$. As $\mathrm{L}^{p}\left(\mathbb{R}^{d}\right)$ also has Pisier's property $(\alpha)$, Theorem 4.4 .4 yields the following multiplier result.

Theorem 5.1.8. Consider the situation of Theorem 5.1.5. Let $r \in[1,2]$, and let $v$ : $\mathbb{R} \rightarrow[1, \infty)$ be an admissible weight with

$$
\frac{(1+|\mathbf{s}|)^{k_{d}}}{v} \in \mathrm{L}^{r}(\mathbb{R}) .
$$

Then $A_{p}$ has a $\gamma$-bounded $\operatorname{Hör}_{v}^{2}\left(\overline{\mathrm{S}}_{\omega_{a}}^{*}\right)$-calculus.

In terms of classical Hörmander spaces we obtain the following corollary.

Corollary 5.1.9. Consider the situation of Theorem 5.1.5. Then $A_{p}$ has a $\gamma$-bounded Hör $^{\beta, 2}\left(\overline{\mathrm{S}}_{\omega_{a}}^{*}\right)$-calculus for each $\beta>k_{d}+\frac{1}{2}=\left\lfloor\frac{d}{2}\right\rfloor+\frac{3}{2}$. 


\subsection{Generators of Symmetric Contraction Semigroups}

Throughout this section, let $(\Omega, \mu)$ be a $\sigma$-finite measure space.

Definition 5.2.1. Using the abbreviation $\mathrm{L}^{1}:=\mathrm{L}^{1}(\Omega, \mu)$ and $\mathrm{L}^{\infty}:=\mathrm{L}^{\infty}(\Omega, \mu)$, a family of linear operators

$$
T_{t}: \mathrm{L}^{1} \cap \mathrm{L}^{\infty} \rightarrow \mathrm{L}^{1}+\mathrm{L}^{\infty} \quad(t \geq 0)
$$

is called a symmetric contraction semigroup if it has the following properties:

1) $T_{t} T_{s}=T_{t+s}$ for all $s, t \geq 0$;

2) $\left\|T_{t} f\right\|_{1} \leq\|f\|_{1}$ and $\left\|T_{t} f\right\|_{\infty} \leq\|f\|_{\infty}$ for all $f \in \mathrm{L}^{1} \cap \mathrm{L}^{\infty}$ and $t \geq 0$;

3) $\left\|T_{t} f-f\right\|_{p} \stackrel{t \searrow 0}{\longrightarrow} 0$ for each $f \in \mathrm{L}^{1} \cap \mathrm{L}^{\infty}$ and $p \in[1, \infty)$;

4) $\int_{\Omega} T_{t} f \cdot \bar{g} \mathrm{~d} \mu=\int_{\Omega} f \cdot \overline{T_{t} g} \mathrm{~d} \mu$ for all $f, g \in \mathrm{L}^{1} \cap \mathrm{L}^{\infty}$ and $t \geq 0$.

For the remainder of the section, we also fix a symmetric contraction semigroup $\left(T_{t}\right)_{t \geq 0}$.

It is an easy consequence of the Riesz-Thorin interpolation theorem that each symmetric contraction semigroup extends to a $C_{0}$-semigroup on $\mathrm{L}^{p}(\Omega, \mu)$ for every $1 \leq p<$ $\infty$. We denote the respective generator by $-A_{p}$. By abuse of notation, we also denote the injective part of $A_{p}$ by $A_{p}$ for $p>1$.

Now fix $p \in(1, \infty)$. The operators $A_{p}$ have a rich history and their holomorphic calculus has been studied by many authors. In 1983, Cowling showed that $A_{p}$ has a bounded $\mathrm{H}^{\infty}\left(\mathrm{S}_{\theta}\right)$-calculus for each angle $\theta \in\left(\omega_{p}^{\mathrm{C}}, \pi\right)$, where

$$
\omega_{p}^{\mathrm{C}}:=\pi\left|\frac{1}{p}-\frac{1}{2}\right|
$$

(see [12, Theorem 3]). In 2003, Kunstmann and Štrkalj improved Cowling's result in the special case that each semigroup operator $T_{t}$ is positive. They showed that $A_{p}$ has a bounded $\mathrm{H}^{\infty}\left(\mathrm{S}_{\theta}\right)$-calculus for every angle

$$
\theta>\omega_{p}^{\mathrm{K}, \mathrm{S}}:=\frac{\pi}{2}\left|\frac{1}{p}-\frac{1}{2}\right|+\left(1-\left|\frac{1}{p}-\frac{1}{2}\right|\right) \arcsin \left(\frac{|p-2|}{2 p-|p-2|}\right)
$$

(see [37]). In 2011, this was extended to general generators of symmetric contraction semigroups by Kriegler ([33, Remark 2]). Finally, in 2017, Carbonaro and Dragičević showed that as a consequence of their multiplier result,

$$
\theta>\omega_{p}^{\mathrm{C}, \mathrm{D}}:=\omega_{p}:=\arcsin \left|1-\frac{2}{p}\right|
$$

suffices. The angle $\omega_{p}$ is sharp in the sense that the Ornstein-Uhlenbeck operator does not admit a bounded $\mathrm{H}^{\infty}$-calculus for any angle smaller than $\omega_{p}$ (see [17, Theorem 2]). 
Remark 5.2.2. One can show that the injective part of a sectorial operator (on a reflexive Banach space) always has dense domain and range (see [23, Proposition 2.3.13.a)]). Hence, by Remark 5.1.6.2), every operator $A_{p}$ has the (BIP) property.

Carbonaro and Dragičević's multiplier result is based on the following estimate, which is part of [6, Proposition 11].

Theorem 5.2.3 (Carbonaro-Dragičević). Let $1<p<\infty$. With the notation from above, there is a constant $c \geq 0$ such that

$$
\left\|A_{p}^{-\mathrm{i} s} f\right\|_{p} \leq c(1+|s|)^{\frac{1}{2}} \mathrm{e}^{\omega_{p}|s|}\|f\|_{p}
$$

for all $f \in \overline{\operatorname{ran} A_{p}}$ and $s \in \mathbb{R}$.

Now Theorem 4.4.4 yields the following improved version of [6, Theorem 1].

Theorem 5.2.4. Let $1<p<\infty, r \in[1,2]$, and let $v: \mathbb{R} \rightarrow[1, \infty)$ be an admissible weight with

$$
\frac{(1+|\mathbf{s}|)^{\frac{1}{2}}}{v} \in \mathrm{L}^{r}(\mathbb{R}) .
$$

Then, $A_{p}$ has a $\gamma$-bounded $\mathrm{Hör}_{v, *}^{2}\left(\overline{\mathrm{S}}_{\omega_{p}}^{*}\right)$-calculus.

In terms of classical Hörmander spaces one obtains the following corollary.

Corollary 5.2.5. Let $1<p<\infty$. Then $A_{p}$ has a $\gamma$-bounded $\mathrm{Hör}_{*}^{\beta, 2}\left(\overline{\mathrm{S}}_{\omega_{p}}^{*}\right)$-calculus for each $\beta>1$.

\subsection{The Ornstein-Uhlenbeck Operator}

In view of Theorem 4.4.4, the better the estimates on the imaginary powers of a given operator are, the better the concluded Hörmander calculus gets. Consider the OrnsteinUhlenbeck operator for example: For $1<p<\infty$ and $d \in \mathbb{N}$ let $\mathcal{L}$ denote the closure of

$$
-\frac{1}{2} \Delta+\mathbf{x} \cdot \nabla
$$

in $\mathrm{L}^{p}\left(\mathbb{R}^{d}, \gamma_{d}\right)$, where $\Delta$ is the Laplacian on $\mathbb{R}^{d}, \nabla$ is the Nabla operator on $\mathbb{R}^{d}$, and $\gamma_{d}$ is the standard Gaussian measure on $\mathbb{R}^{d}$, i.e.,

$$
\gamma_{d}(\mathrm{~d} x)=\frac{1}{(2 \pi)^{d / 2}} \mathrm{e}^{-\frac{|x|^{2}}{2}} \mathrm{~d} x .
$$

In [39], Mauceri, Meda, and Sjögren proved the following estimate, where by abuse of notation we also write $\mathcal{L}$ for the injective part of $\mathcal{L}$.

Theorem 5.3.1. Let $1<p<\infty$. Then there is a constant $c \geq 0$ such that

$$
\left\|\mathcal{L}^{-\mathrm{i} s} f\right\|_{p} \leq c \mathrm{e}^{\omega_{p}|s|}\|f\|_{p}
$$

for all $f \in \overline{\operatorname{ran} \mathcal{L}}$ and $s \in \mathbb{R}$. 
Hence, Theorem 4.4 .4 yields the following result.

Theorem 5.3.2. Let $1<p<\infty$, and let $v: \mathbb{R} \rightarrow[1, \infty)$ be an admissible weight. Then $\mathcal{L}$ has a $\gamma$-bounded $\mathrm{Hör}_{v, *}^{2}\left(\overline{\mathrm{S}}_{\omega_{p}}^{*}\right)$-calculus on $\mathrm{L}^{p}\left(\mathbb{R}^{d}, \gamma_{d}\right)$.

In terms of classical Hörmander spaces one obtains the following corollary.

Corollary 5.3.3. Let $1<p<\infty$. Then $\mathcal{L}$ has a $\gamma$-bounded Hör ${ }^{\beta, 2}\left(\overline{\mathrm{S}}_{\omega_{p}}^{*}\right)$-calculus on $\mathrm{L}^{p}\left(\mathbb{R}^{d}, \gamma_{d}\right)$ for each $\beta>\frac{1}{2}$.

Remark 5.3.4. Theorem 5.3.2 is the best result we can expect for an operator within our theory. This result is optimal in the sense that, on one hand, every admissible weight leads to a $\gamma$-bounded Hörmander calculus on the sector $\mathrm{S}_{\omega_{p}}$. On the other hand, the angle $\omega_{p}$ cannot be improved, since it is known that $\mathcal{L}$ does not even admit a bounded $\mathrm{H}^{\infty}\left(\mathrm{S}_{\theta}\right)$-calculus for any angle $\theta<\omega_{p}$ (see [17, Theorem 2]). Hence, $\mathcal{L}$ cannot admit a bounded Hörmander calculus on any sector smaller than $\mathrm{S}_{\omega_{p}}$ either. 


\section{Bibliography}

[1] G. Alexopoulos. Spectral multipliers on Lie groups of polynomial growth. Proc. Amer. Math. Soc., 120 (3) (1994), 973-979.

[2] J.-P. Anker. $\mathbf{L}_{p}$ Fourier multipliers on Riemannian symmetric spaces of the noncompact type. Ann. of Math. (2), 132 (3) (1990), 597-628.

[3] J.-P. Anker, N. Lohoué. Multiplicateurs sur certains espaces symétriques. Amer. J. Math., 108 (6) (1986), 1303-1353.

[4] C. Batty, M. Haase, J. Mubeen. The holomorphic functional calculus approach to operator semigroups. Acta Sci. Math. (Szeged), 79 (1-2) (2013), 289-323.

[5] S. Blunck. A Hörmander-type spectral multiplier theorem for operators without heat kernel. Ann. Sc. Norm. Super. Pisa Cl. Sci. (5), 2 (3) (2003), 449-459.

[6] A. Carbonaro, O. Dragičević. Functional calculus for generators of symmetric contraction semigroups. Duke Math. J., 166 (5) (2017), 937-974.

[7] M. Christ. $L^{p}$ bounds for spectral multipliers on nilpotent groups. Trans. Amer. Math. Soc., 328 (1) (1991), 73-81.

[8] J. L. Clerc, E. M. Stein. $L^{p}$-multipliers for noncompact symmetric spaces. Proc. Nat. Acad. Sci. U.S.A., 71 (1974), 3911-3912.

[9] G. M. Constantine, T. H. Savits. A multivariate Faà di Bruno formula with applications. Trans. Amer. Math. Soc., 348 (2) (1996), 503-520.

[10] M. Cowling, I. Doust, A. McIntosh, A. Yagi. Banach space operators with a bounded $H^{\infty}$ functional calculus. J. Austral. Math. Soc. Ser. A, 60 (1) (1996), 51-89.

[11] M. Cowling, S. Meda. Harmonic analysis and ultracontractivity. Trans. Amer. Math. Soc., 340 (2) (1993), 733-752.

[12] M. G. Cowling. Harmonic analysis on semigroups. Ann. of Math. (2), 117 (2) (1983), 267-283.

[13] K.-J. Engel, R. Nagel. One-parameter semigroups for linear evolution equations, volume 194 of Graduate Texts in Mathematics. Springer, New York (2000). 
[14] F. Herbert. Geometric measure theory. Volume 153 of Grundlehren der mathematischen Wissenschaften. Springer, New York (1969).

[15] G. B. Folland, E. M. Stein. Hardy spaces on homogeneous groups. volume 28 of Mathematical Notes. Princeton University Press, Princeton, N.J., University of Tokyo Press, Tokyo (1982).

[16] M. Frazier, B. Jawerth, G. Weiss. Littlewood-Paley theory and the study of function spaces, volume 79 of CBMS Regional Conference Series in Mathematics. Published for the Conference Board of the Mathematical Sciences, Washington, DC; by the American Mathematical Society, Providence, RI (1991).

[17] J. García-Cuerva, G. Mauceri, S. Meda, P. Sjögren, J. L. Torrea. Functional calculus for the Ornstein-Uhlenbeck operator. J. Funct. Anal., 183 (2) (2001), 413-450.

[18] J. García-Cuerva, G. Mauceri, P. Sjögren, J. L. Torrea. Spectral multipliers for the Ornstein-Uhlenbeck semigroup. J. Anal. Math., 78 (1999), 281-305.

[19] L. Grafakos. Classical Fourier analysis, volume 249 of Graduate Texts in Mathematics. Springer, New York, second edition (2008).

[20] L. Grafakos. Modern Fourier analysis, volume 250 of Graduate Texts in Mathematics. Springer, New York, second edition (2009).

[21] B. H. Haak, M. Haase. Square Function Estimates and Functional Calculi. Unpublished Manuscript (2017) http://www.math.u-bordeaux.fr/ bhaak/HaHa-sqf.pdf (05.05.2017).

[22] M. Haase. A general framework for holomorphic functional calculi. Proc. Edinb. Math. Soc. (2), 48 (2) (2005), 423-444.

[23] M. Haase. The functional calculus for sectorial operators, volume 169 of Operator Theory: Advances and Applications. Birkhäuser, Basel (2006).

[24] M. Haase. Lectures on Functional Calculus. Lecture notes of the 21st Internet Seminar (2018) http://www.math.uni-kiel.de/isem21/en/course/phase1/isem21lectures-on-functional-calculus (18.03.2018).

[25] W. Hebisch. A multiplier theorem for Schrödinger operators. Colloq. Math., 60/61 (2) (1990), 659-664.

[26] W. Hebisch. Multiplier theorem on generalized Heisenberg groups. Colloq. Math., 65 (2) (1993), 231-239.

[27] W. Hebisch, G. Mauceri, S. Meda. Holomorphy of spectral multipliers of the Ornstein-Uhlenbeck operator. J. Funct. Anal., 210 (1) (2004), 101-124.

[28] L. Hörmander. Estimates for translation invariant operators in $L^{p_{\text {spaces. }} \text { Acta }}$ Math., 104 (1960), 93-140. 
[29] L. Hörmander. The analysis of linear partial differential operators. I, volume 256 of Grundlehren der Mathematischen Wissenschaften. Springer, Berlin, second edition (1990).

[30] G. Hu. Spectral multipliers on exponentially locally doubling metric measure spaces. Ann. Mat. Pura Appl. (4), 197 (4) (2018), 1151-1173.

[31] T. Hytönen, J. van Neerven, M. Veraar, L. Weis. Analysis in Banach spaces. Vol. II, volume 67 of Ergebnisse der Mathematik und ihrer Grenzgebiete. 3. Folge. A Series of Modern Surveys in Mathematics [Results in Mathematics and Related Areas. 3rd Series. A Series of Modern Surveys in Mathematics]. Springer, Cham (2017).

[32] C. Kriegler. Spectral Multipliers, R-bounded Homomorphisms, and Analytic Diffusion Semigroups. doctoral thesis, Karlsruhe Institut of Technology (2009) http://publikationen.bibliothek.kit.edu/1000015866/1203560

[33] C. Kriegler. Analyticity angle for non-commutative diffusion semigroups. J. Lond. Math. Soc. (2), 83 (1) (2011), 168-186.

[34] C. Kriegler. Hörmander functional calculus for Poisson estimates. Integral Equations Operator Theory, 80 (3) (2014), 379-413.

[35] C. Kriegler, L. Weis. Spectral multiplier theorems and averaged $R$-boundedness. Semigroup Forum, 94 (2) (2017), 260-296.

[36] C. Kriegler, L. Weis. Spectral multiplier theorems via $H^{\infty}$ calculus and $R$-bounds. Math. Z., 289 (1-2) (2018), 405-444.

[37] P. C. Kunstmann, v. Štrkalj. $H^{\infty}$-calculus for submarkovian generators. Proc. Amer. Math. Soc., 131 (7) (2003), 2081-2088.

[38] G. Mauceri, S. Meda. Vector-valued multipliers on stratified groups. Rev. Mat. Iberoamericana, 6 (3-4) (1990), 141-154.

[39] G. Mauceri, S. Meda, P. Sjögren. Sharp estimates for the Ornstein-Uhlenbeck operator. Ann. Sc. Norm. Super. Pisa Cl. Sci. (5), 3 (3) (2004), 447-480.

[40] A. McIntosh. Operators which have an $H_{\infty}$ functional calculus. In Miniconference on operator theory and partial differential equations (North Ryde, 1986), volume 14 of Proc. Centre Math. Anal. Austral. Nat. Univ., Austral. Nat. Univ., Canberra (1986), 210-231.

[41] S. Meda. A general multiplier theorem. Proc. Amer. Math. Soc., 110 (3) (1990), 639-647.

[42] S. G. Mikhlin. On the multipliers of Fourier integrals. Dokl. Akad. Nauk SSSR (N.S.), 109 (1956), 701-703. 
[43] D. Müller. Functional calculus on Lie groups and wave propagation. In Proceedings of the International Congress of Mathematicians, Vol. II, number Extra Vol. II, Berlin (1998), 679-689.

[44] D. Müller and E. M. Stein. On spectral multipliers for Heisenberg and related groups. J. Math. Pures Appl. (9), 73 (4) (1994), 413-440.

[45] R. Remmert, G. Schumacher. Funktionentheorie 1, 5. neu bearb. aufl. edition. Springer, Berlin (2002).

[46] W. Rudin. Real and complex analysis, third edition. McGraw-Hill Book Co., New York (1987).

[47] E. Sasso. Functional calculus for the Laguerre operator. Math. Z., 249 (3) (2005), 683-711.

[48] R. J. Stanton, P. A. Tomas. Expansions for spherical functions on noncompact symmetric spaces. Acta Math., 140 (3-4) (1978), 251-276.

[49] E. M. Stein. Topics in harmonic analysis related to the Littlewood-Paley theory. Annals of Mathematics Studies, No. 63. Princeton University Press, Princeton, N.J., University of Tokyo Press, Tokyo (1970).

[50] R. S. Strichartz. Multipliers on fractional Sobolev spaces. J. Math. Mech., 16 (1967), 1031-1060.

[51] R. S. Strichartz. A note on Sobolev algebras. Proc. Amer. Math. Soc., 29 (1971), 205-207.

[52] K. Stromberg. An elementary proof of Steinhaus's theorem. Proc. Amer. Math. Soc., 36 (1972), 308.

[53] M. E. Taylor. $L^{p}$-estimates on functions of the Laplace operator. Duke Math. J., 58 (3) (1989), 773-793.

[54] X. Thinh Duong, E. M. Ouhabaz, A. Sikora. Plancherel-type estimates and sharp spectral multipliers. J. Funct. Anal., 196 (2) (2002), 443-485. 


\section{Index}

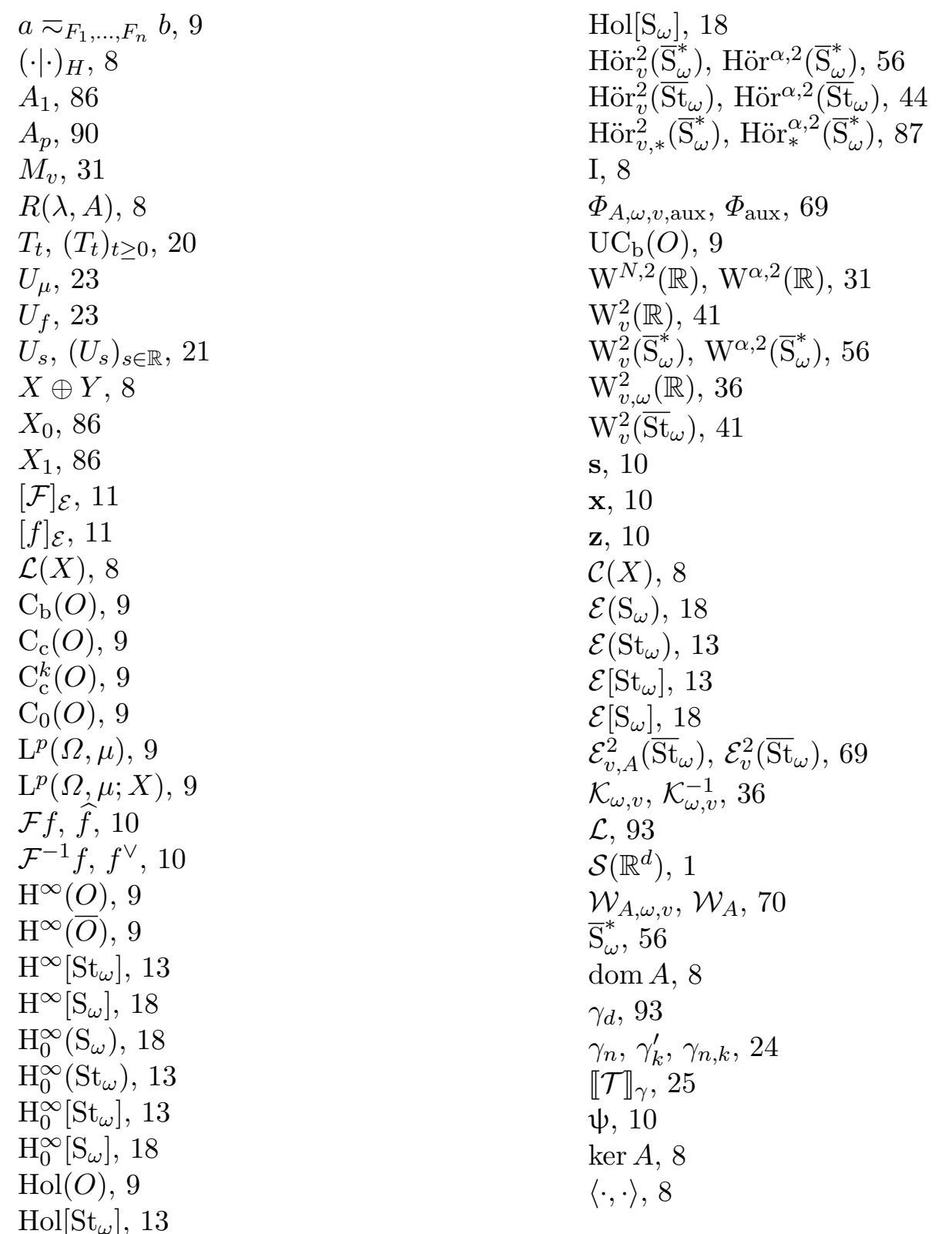


$\mathbb{1}_{D}, 10$

(BIP), 20

$\omega_{\text {se }}(A), 18$

$\omega_{\mathrm{st}}(A), 13$

$\omega_{a}, 89$

ran $A, 8$

$\rho(A), 8$

$\mathrm{S}_{\omega}, 8$

$\sigma(A), 8$

$\mathrm{St}_{\omega}, 8$

$\operatorname{supp}(f), 9$

$\tau_{w}, 10$

$\theta(U), 21$

$\mathrm{C}^{k}(O), 9$

$a \lesssim F_{1}, \ldots, F_{n} b, 9$

$a \sim A, 90$

$f * g, 10$

$f^{*}, \psi^{*}, 10$

$f_{r}, \psi_{r}, 10$

$r_{k}, 24$

admissible

function, 31

weight, 31

algebra homomorphism

$R$-bounded, 26

$\gamma$-bounded, 26

closable, 69

non-degenerate, 11

unbounded, 69

algebraic extension, 11

algebraic extension procedure, 11

anchor

element, 11

set, 11

anchored, 11

angle of sectoriality, 18

auxiliary calculus, 69

extended, 70

bound, $\gamma_{-}, 25$

bounded

$$
\begin{aligned}
& R-, 26 \\
& \gamma-, 25
\end{aligned}
$$

bounded imaginary powers, 20

Composition rule extended Sobolev calculus, 85

holomorphic calculus, 20

Convergence lemma

auxiliary calculus, 74

Hörmander calculus, 66

holomorphic calculus, 15

weak version, 74

cotype

finite, 24

cotype, Rademacher, 24

dense domain and range, 90

elementary calculus, 11

elementary Sobolev function, 69

Fourier-Stieltjes calculus, 23

generalized

Hörmander space

on a sector, 56

on a strip, 44

Sobolev space

on a sector, 56

on a strip, 41

generator, 21

group type, 21

group, $C_{0^{-}}, 21$

Hörmander calculus

sectorial, 84

bounded, 84

sectorial, non-injective, 87

bounded, 87

strip type, 65

bounded, 65

holomorphic calculus

bounded

sectorial, 19

strip, 16

elementary calculus

sectorial, 18 
strip, 14

extended

sectorial, 19

strip, 15

injective part, 86

Main result

sectorial version, 85

strip version, 75

operator

0-sectorial, 18

0-strip type, 13

sectorial, 18

strip type, 13

polynomial

homogeneous of degree $m, 89$

strongly elliptic, 89

property $(\alpha), 24$

Rademacher

boundedness, 26

variable, 24

semigroup

$C_{0^{-}}, 20$

symmetric contraction, 92

set

$R$-bounded, 26

$\gamma$-bounded, 25

Sobolev anchored, 70

densely, 74

Sobolev calculus

sectorial

bounded, 84

extended, 84

sectorial, non-injective

extended, 87

strip type

bounded, 60

extended, 64

standard Gaussian, 24

strip type, 13 total range, 12

type, Rademacher, 24 


\section{Erklärung}

Hiermit erkläre ich, dass ich die vorliegende Arbeit abgesehen von der Beratung durch den Betreuer meiner Promotion unter Einhaltung der Regeln guter wissenschaftlicher Praxis der Deutschen Forschungsgemeinschaft selbstständig angefertigt habe, keine anderen als die angegebenen Hilfsmittel verwendet habe, und dass mir noch nie ein akademischer Grad entzogen wurde.

Es ist geplant, die Hauptresultate dieser Arbeit im Rahmen eines mit meinem Betreuer gemeinsam verfassten (derzeit noch nicht finalisierten) Artikels zu veröffentlichen.

Kiel, den 06.08.2019

(Florian Pannasch) 